\title{
Multi-Camera Active-Vision for Markerless Shape Recovery of Unknown Deforming Objects
}

\author{
by
}

Evgeny Nuger

A thesis submitted in conformity with the requirements

for the degree of Doctor of Philosophy

Department of Mechanical and Industrial Engineering

University of Toronto

(C) Copyright by Evgeny Nuger 2017 


\title{
Multi-Camera Active-Vision for Markerless Shape Recovery of Unknown Deforming Objects
}

\author{
Evgeny Nuger \\ Doctor of Philosophy \\ Department of Mechanical and Industrial Engineering \\ University of Toronto
}

2017

\section{Abstract}

This thesis proposes a multi-camera active-vision reconfiguration system which selects camera poses online to improve the shape recovery of a priori unknown, markerless, deforming objects in dynamic environments. The objectives of shape recovery are defined as surface sampling accuracy, and shape completeness. The completeness objective is generalized for both solid and surface-based objects as the maximization of surface visibility. Thus, improving the recovered shape of target objects is shown to be analogous to maximizing the surface area visibility. This improvement is achieved through the on-line reconfiguration of multiple cameras. The system developed herein is composed of a shape recovery method, a robust tracking algorithm, and a multi-camera reconfiguration method.

The shape recovery method is based on a modular fusion technique that produces a complete 3D mesh-model of the target object. The method fuses triangulation data with a visual hull to maximize recovery accuracy. The modularity of the method lies in the ability to modify data filtering techniques to improve modelling accuracy, and interchange stereo correspondence features. The adaptive particle filtering algorithm produces a deformation estimation of the recovered model from the tracking data. The algorithm automatically adapts to the quantity of tracking data available and changes in the object's dynamics. The modularity of the algorithm allows modifications in terms of the number of particles and motion models for task-specific implementations. The reconfiguration method consists of a robust stereovisibility objective function, workspace discretization, and a path planner. The complete system can 
improve the shape recovery of a priori unknown deforming objects in obstacle-laden environments when compared to static camera methods.

To validate the proposed system, extensive simulations and experiments were conducted. The simulations tested the system in comparison to static multi-camera systems, and ideal camera placement where the object model was a priori known and the camera's dynamics were unconstrained. Simulation results showed the proposed methodology outperformed static cameras and approached the performance of ideal camera placement in a dynamic, obstacle-laden environment. The experimental results showed similar improvement in shape recovery when comparison to a static camera system in an obstacle-laden environment. 


\section{Acknowledgments}

I would like to firstly thank my supervisor, Professor Beno Benhabib, for his continuous support through my thesis program, helpful advice, and guidance. I would also like to thank the professors on my thesis committee, Prof. J.K. Mills and Prof. G. Nejat for their valuable input, and help developing the dissertation.

I would like to thank all my colleagues and friends from the Computer Integrated Manufacturing Laboratory and the Autonomous System and Biomechatronics Laboratory for their assistance: Dr. Adam Le, Dr. Masih Mahmoodi, Dr. Hay Azulay, Dr. Veronica Marin, Dr. Matthew Mackay, Mr. Jeremy Chan, Mr. Arta Alagheband, Dr. Ashish Macwan, Mr. Mario Luces, Mr. Julio Vilela, Mr. Ray Zhao, Mr. Zendai Kashino, and Mr. Justin Kim.

I would like to especially thank Mr. David Schacter and Professor Pinar Boyraz for their insight and mentorship. I would also like to thank Mr. Benjamin Corcoran for his work on the camera control code, Mr. Robert Morettin for his contribution to the experimental setup design, and Mr. Edson Nakada, Mr. Pedro De Oliveria, and Mr. Elvis Rocha Lima for their assistance in preparing the experimental platform.

To my parents, Max Noger and Albina Noger, and my friends, I am eternally grateful for your continued encouragement, advice, and support.

Lastly, I gratefully acknowledge the financial support of the Natural Science and Engineering Research Council, and the University of Toronto Graduate Fellowship program for their gracious funding. 


\section{Table of Contents}

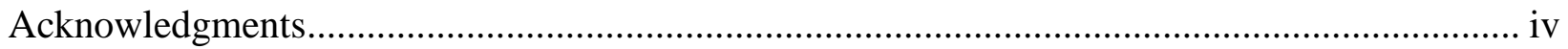

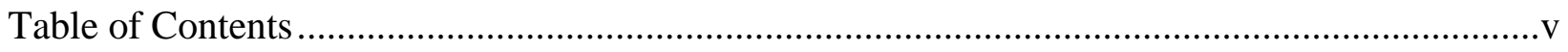

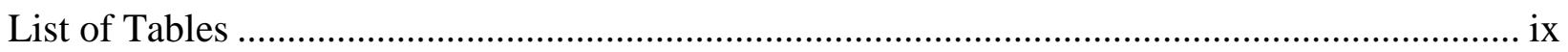

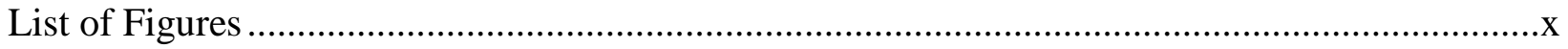

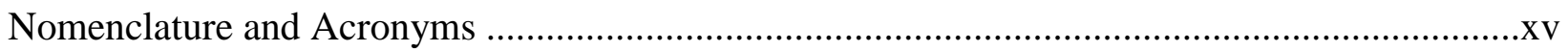

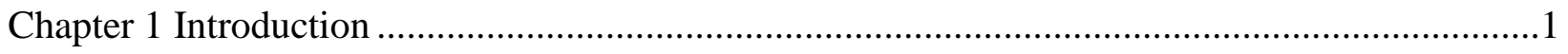

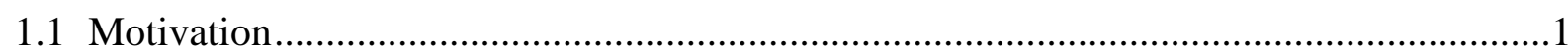

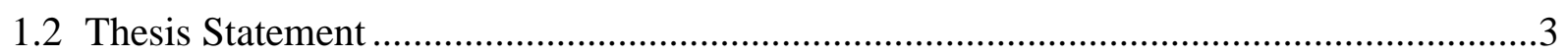

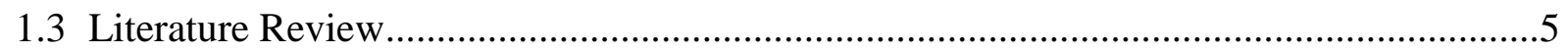

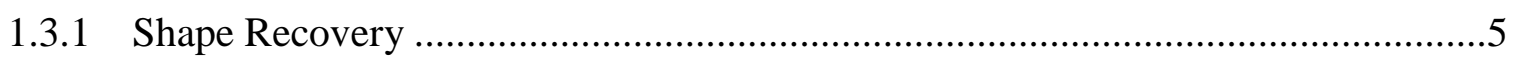

1.3.2 Computer-Vision Tracking .......................................................................

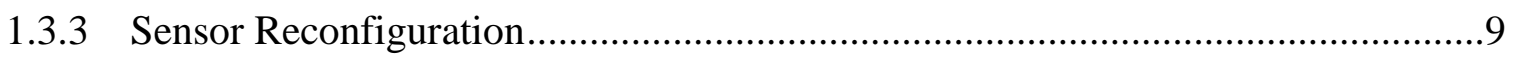

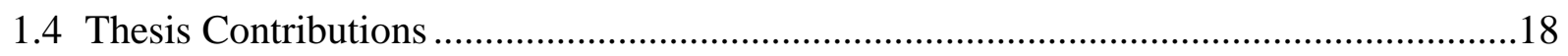

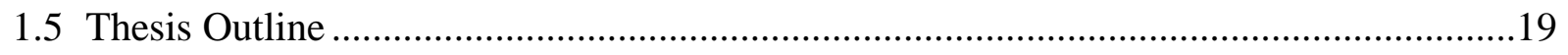

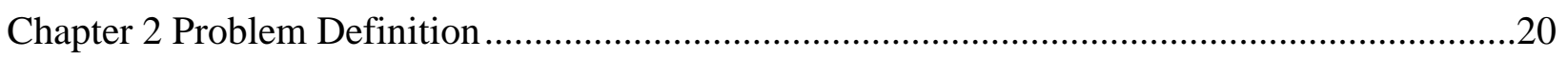

2.1 Generalization of the Shape Recovery Objectives.................................................20

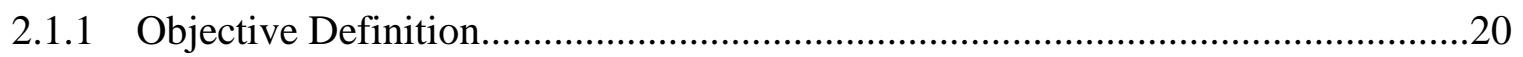

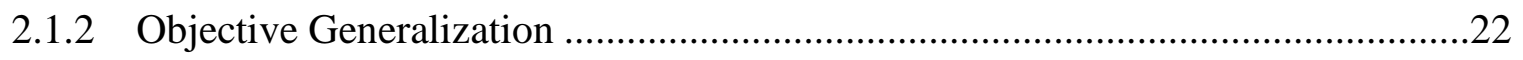

2.2 Application of the Generalized Shape Recovery Objectives ......................................23

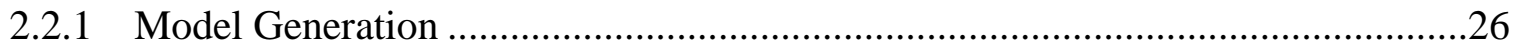

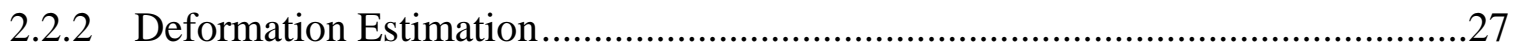

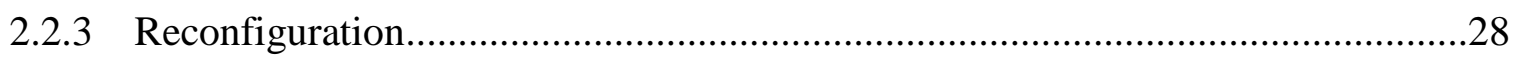

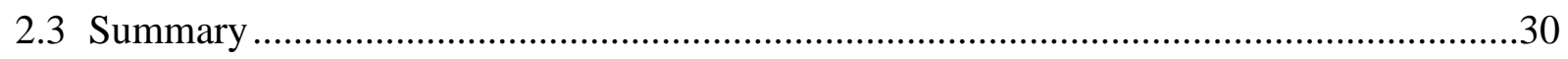

Chapter 3 Methodology: Model Generation .................................................................. 31

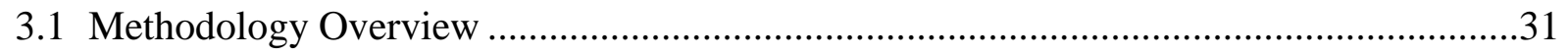




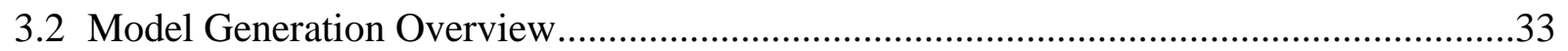

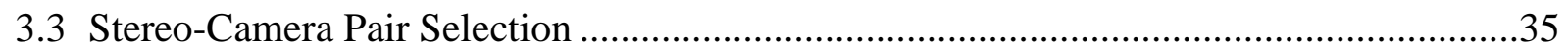

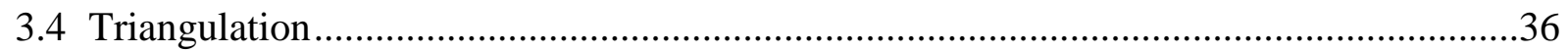

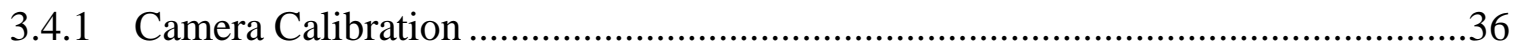

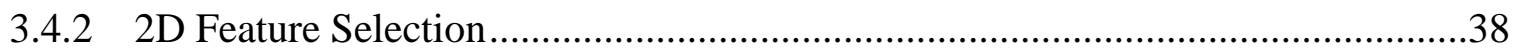

3.4.3 2D Matching and Filtering ................................................................................

3.4.4 Triangulation \& 3D Filtering ............................................................................

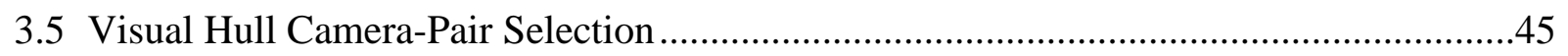

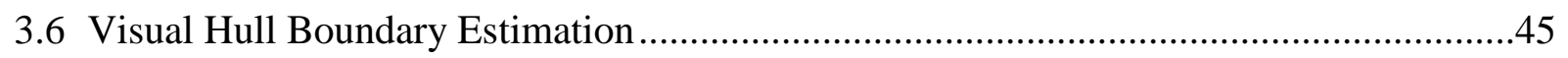

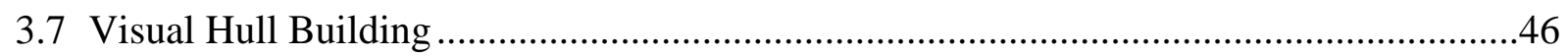

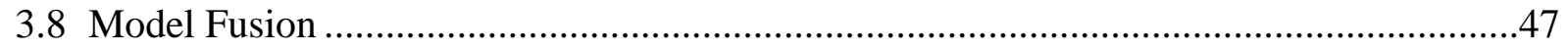

Chapter 4 Methodology: Deformation Estimation ……..............................................................5

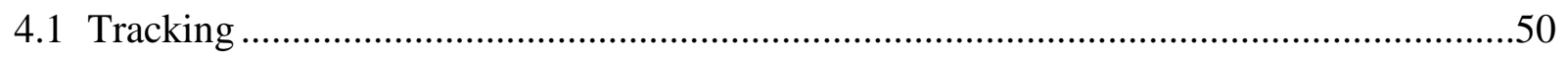

4.1.1 Feature Found Stream ..................................................................................52

4.1.2 Feature Tracked Stream ...............................................................................54

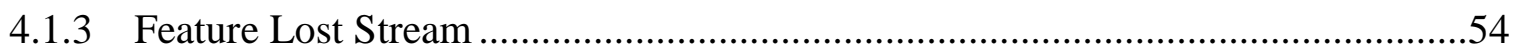

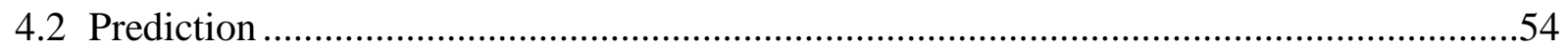

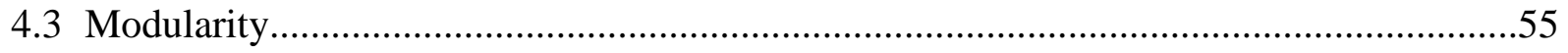

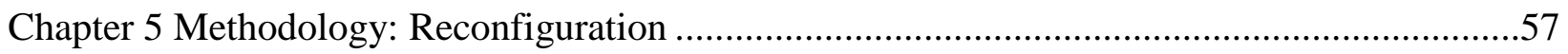

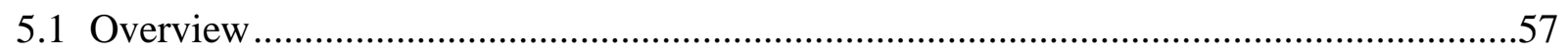

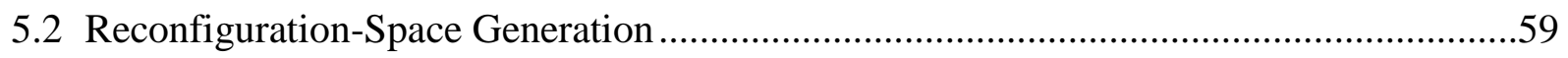

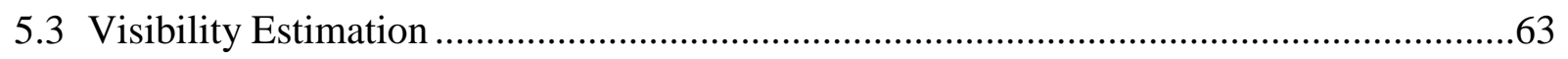

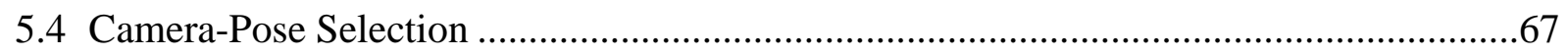

5.5 Camera Path Planning .................................................................................................69

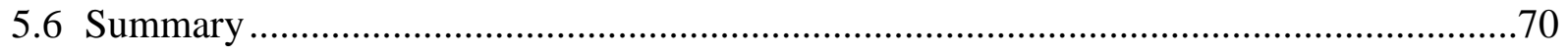

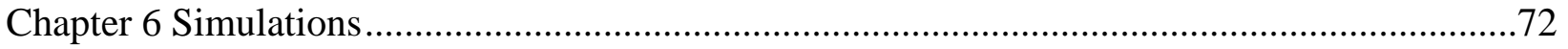




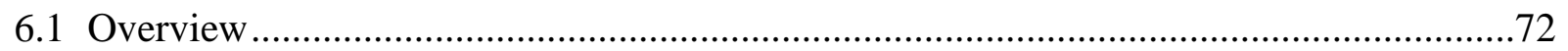

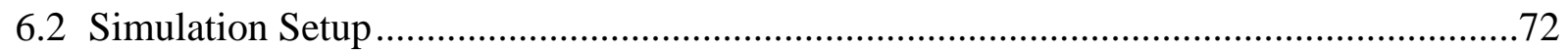

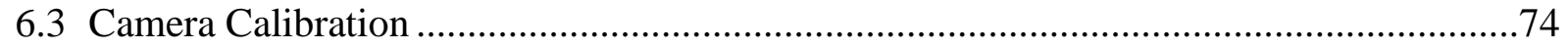

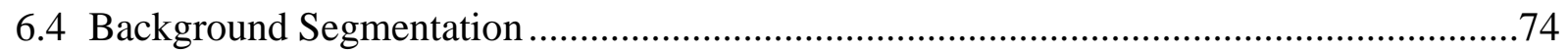

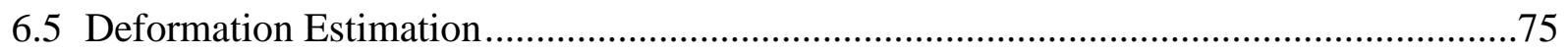

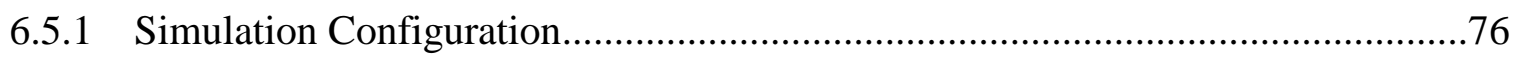

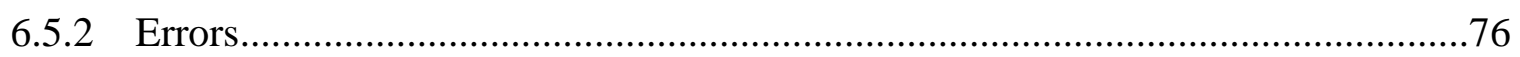

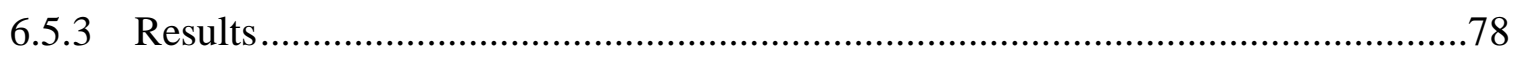

6.6 Model Generation and Deformation Estimation................................................ 84

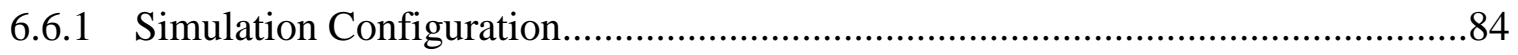

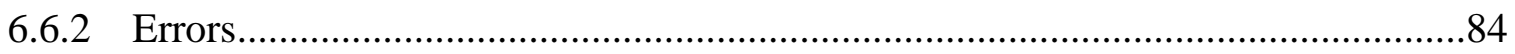

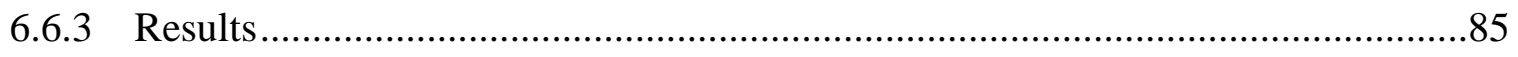

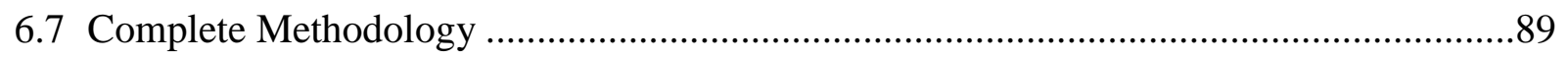

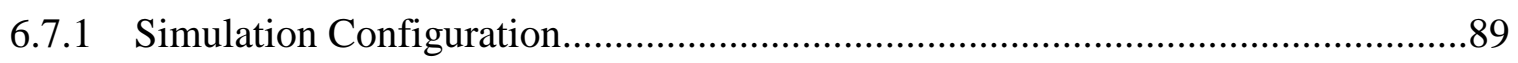

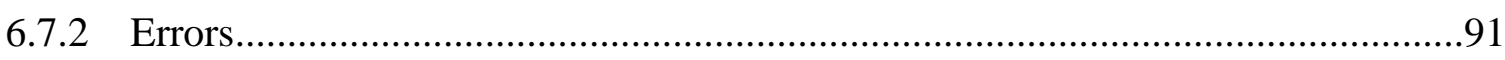

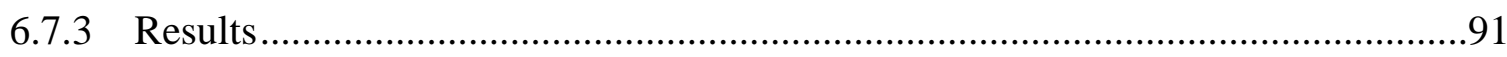

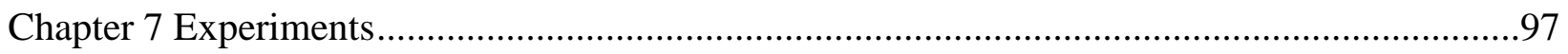

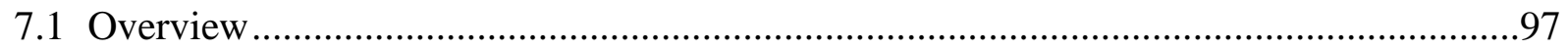

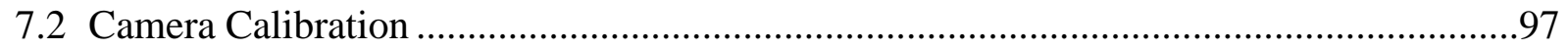

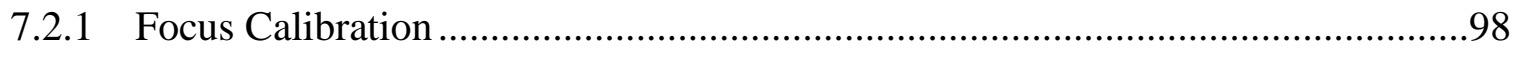

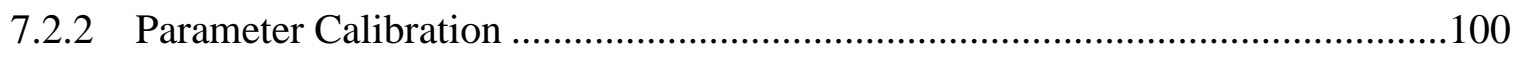

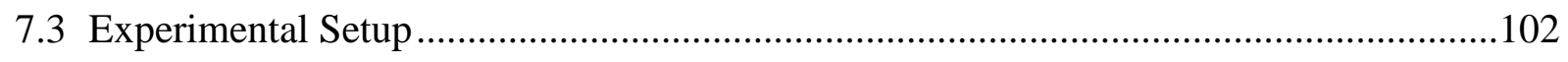

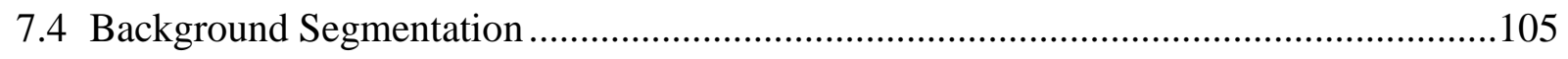

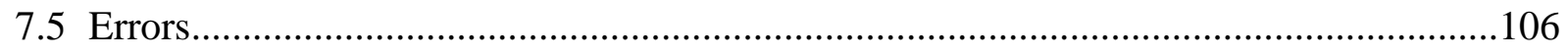

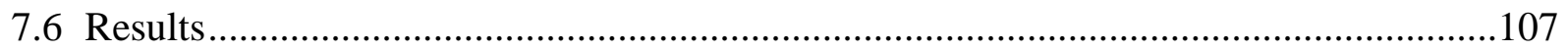

Chapter 8 Conclusions and Future Work ......................................................................... 114 


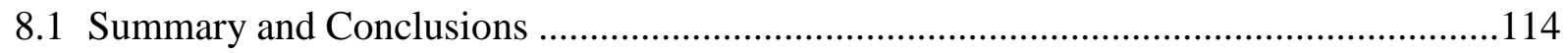

8.1.1 Modular, Multi-Camera Active-Vision System.....................................................116

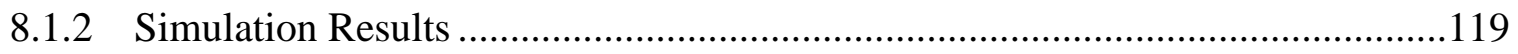

8.1.3 Experimental Results ..............................................................................120

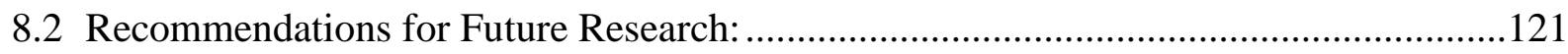

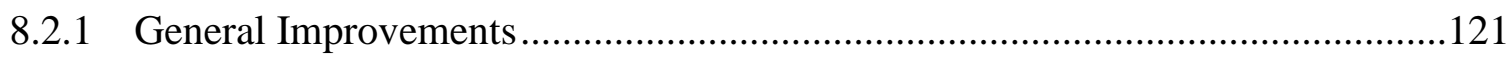

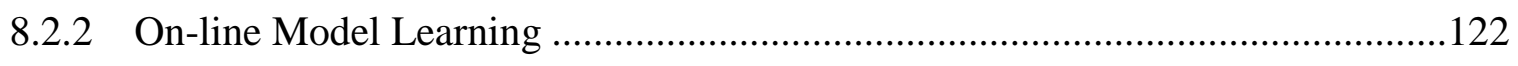

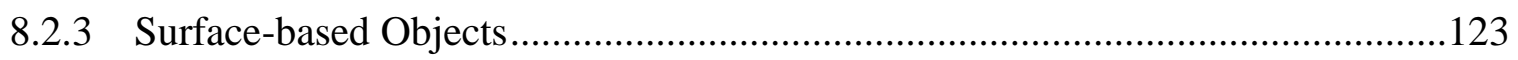

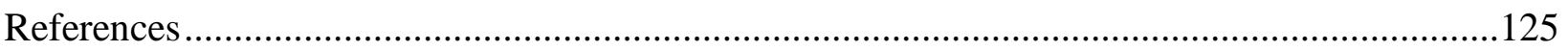

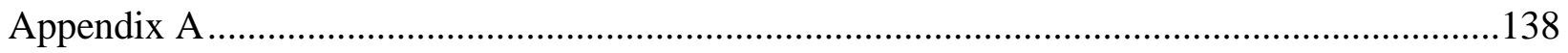

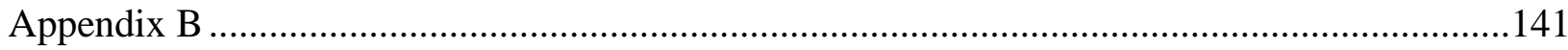

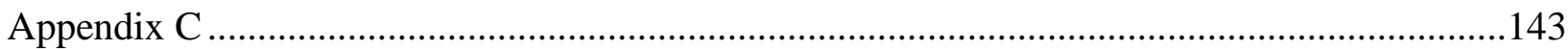

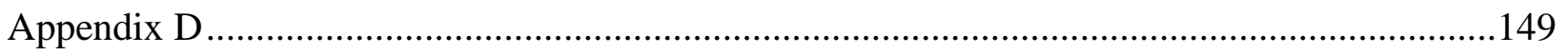

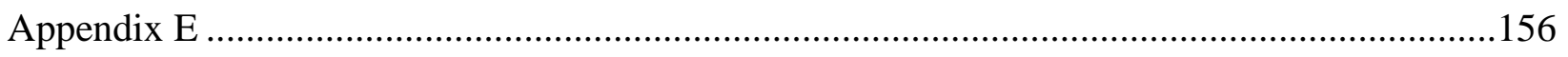

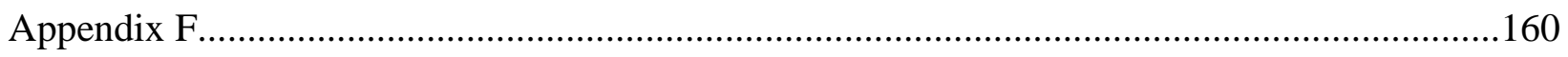

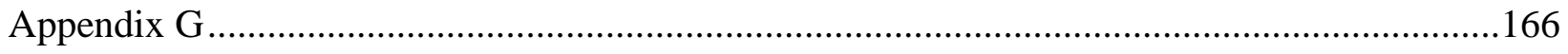




\section{List of Tables}

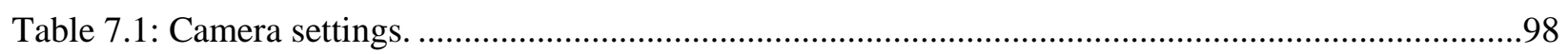

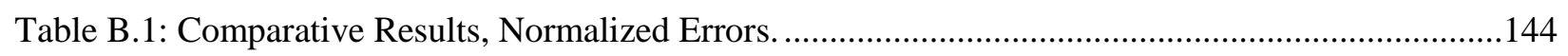

Table B.2: Comparative Results with Known Model......................................................................146 


\section{List of Figures}

Figure 2.1: Volumetric model completeness, left: low, middle: medium, right: high.

Figure 2.2: Projection of the 3D manifold into 2D feature space...

Figure 2.3: Relation of uniform sampling density to completeness of the recovered model, top row: feature space and sample points (red), bottom row: recovered surface patches (red) associated with sampled points.

Figure 2.4: Left: non-uniform feature space sampling, right: associated object patches recovered .25

Figure 2.5: Left: point cloud representation, middle: polygon surface representation, right: voxel representation.

Figure 2.6: Illustration of the camera parameter sets and one camera parameter combination.

Figure 3.1: Proposed methodology 32

Figure 3.2: Example of poorly positioned cameras. .34

Figure 3.3: Model generation methodology. .34

Figure 3.4: Stereo triangulation definition. .35

Figure 3.5: Example of epipolar geometry.

Figure 3.6: Epipolar disparity.

Figure 3.7: Nearest neighbor filter example for stereo-camera pair.

Figure 3.8: Effective sensing range filter for a stereo-camera pair.

Figure 3.9: Surface patch representation of a deformable object with 3 stereo-camera pairs producing 3 surface patches.

Figure 3.10: Visual hull camera pair selection and boundary estimation..............................................46

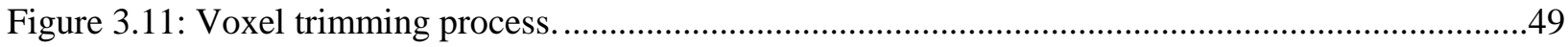

Figure 4.1: Adaptive particle filtering algorithm for deformation estimation......................................51

Figure 5.1 Left: poor camera configuration, right: improved camera configuration................................57

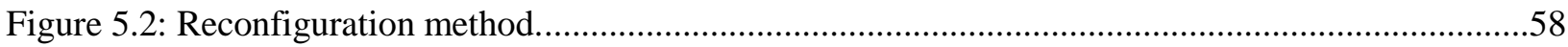


Figure 5.3: Generalized positional combination problem.

Figure 5.4: Logarithm of the difference between the total number of positional combinations for independent placement vectors and generalized vectors.

Figure 5.5: Example of a pseudo-redundant combination.

Figure 5.6: Spherical vector space, left: standard r- $\theta$ increments, right: uniformly distributed vectors from non-standard $\mathrm{r}-\theta$ values.

Figure 5.7: (a) Data storage requirements for index matrix K, when varying the number of positions for different numbers of camera pairs; (b) the total number of combinations. .62

Figure 5.8: Example of ill-positioned vector combination. .62

Figure 5.9: Visibility-estimation process outline. .64

Figure 5.10: Optimal pose selection process. .68

Figure 5.11: Overview of positional and rotational stereo-pair placement for a given positioning vector. 68

Figure 5.12: Image padding. 69

Figure 5.13: Path planning problem visualization .70

Figure 6.1: Simulation framework.

Figure 6.2: Background Separation Process: (a) input image, (b) color thresholding, (c) selection of largest background connected group, (d) Gaussian blurring, (e) thresholding blurred image, and (f) silhouette boundary identification. .75

Figure 6.3: Movie-strip for Simulation 1. .78

Figure 6.4: Left: triangulation, prediction and relative prediction error metrics for each demand instant. Right: total number of tracked and triangulated points for each demand instant. .78

Figure 6.5: Movie-strip of Simulation 2.

Figure 6.6: Left: triangulation, prediction and relative prediction error metrics for each demand instant. Right: total number of tracked and triangulated points for each demand instant. .79

Figure 6.7: Movie-strip of Simulation 3.

Figure 6.8: Left: triangulation, prediction and relative prediction error metrics for each demand instant. Right: total number of tracked and triangulated points for each demand instant .80

Figure 6.9: Movie-strip of Simulation 4. 
Figure 6.10: Left: triangulation, prediction and relative prediction error metrics for each demand instant.

Right: total number of tracked and triangulated points for each demand instant

Figure 6.11: Movie-strip of Simulation 5

Figure 6.12: Left: triangulation, prediction and relative prediction error metrics for each demand instant.

Right: total number of tracked and triangulated points for each demand instant. .82

Figure 6.13: Movie-strip of Simulation 6. .83

Figure 6.14: Left: triangulation, prediction and relative prediction error metrics for each demand instant.

Right: total number of tracked and triangulated points for each demand instant. .83

Figure 6.15: Results for Simulation 'Sph'. .86

Figure 6.16: Normalized Errors for (a) Surface and Volume, and (b) Triangulation and Visual Hull Data.

Figure 6.17: Results for Simulation 'Pr1', Configuration 'II'. .88

Figure 6.18: Normalized Errors for (a) Surface and Volume, and (b) Triangulation and Visual Hull Data.

Figure 6.19: Example camera configuration about the target object...................................................90

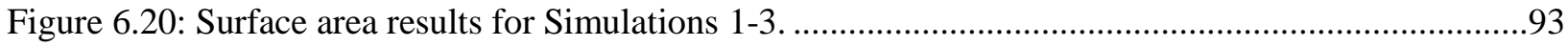

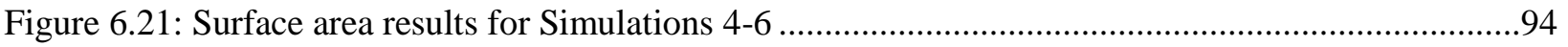

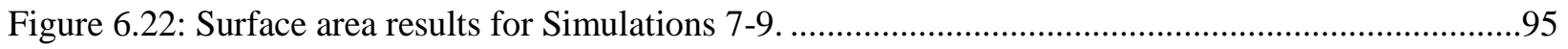

Figure 6.23: Surface area results for Simulations 10-12 .............................................................. 96

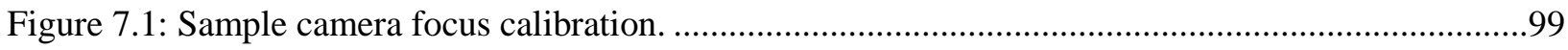

Figure 7.2: Depth of field range for $18 \mathrm{~mm}$ focal length lens........................................................... 100

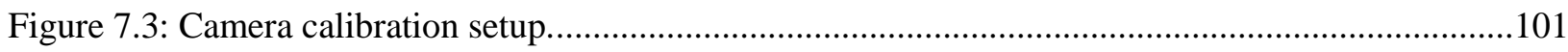

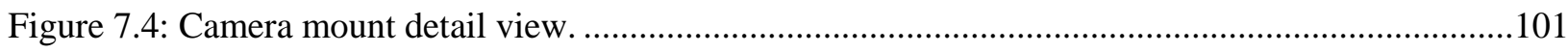

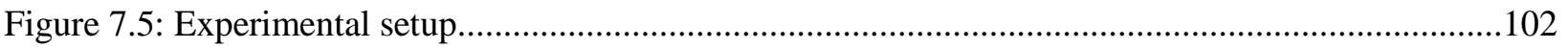

Figure 7.6: Hardware implementation of the experimental setup. .....................................................103

Figure 7.7: Software implementation of the experimental setup....................................................... 104

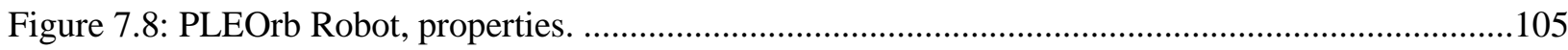


Figure 7.9: Background segmentation through GrabCut, left: input image with guides, right: resulting binary segmented image.

Figure 7.10: Results for Experiments 1-3

Figure 7.11: Results for Experiments 4-5

Figure 7.12: Static camera experiment, Simulation Frames $\{1,3,5,7,9,11\}$, respectively 110

Figure 7.13: Static camera experiment, Simulation Frames $\{13,15,17,19\}$, respectively

Figure 7.14: Reconfiguration experiments through proposed method, Simulation Frames $\{1,3,5,7,9$, $11\}$, respectively.

Figure 7.15: Reconfiguration experiments through proposed method, Simulation Frames $\{13,15,17,19\}$, respectively

Figure 8.1: Limited reconfiguration workspace for surface-based objects. 124

Figure A.1: Input data, left: Scene, right: matching template. 138

Figure A.2: Cross-correlation map. 138

Figure A.3: Resulting cross-correlation feature match 139

Figure A.4: Input data, left: Scene, right: matching template, rotated. 139

Figure A.5: Cross-correlation map.

Figure A.6: SIFT matches from template to scene. 140

Figure A.7: SIFT matches from rotated template to scene. 140

Figure B.1: Results for Simulation Sph, Alternative Viewpoint. 141

Figure B.2: Results for Simulation Pr1, Alternative Viewpoint 142

Figure B.3: Camera Configuration I (left), II (right). 146

Figure B.4: Camera Configuration III (left), IV (right) 146

Figure B.5: Camera Configuration V (left), VI (right)

Figure B.6: Simulation Sph, Top Row - Initial Object Deformation, Bottom Row - Final Object Deformation.

Figure B.7: Simulation Pr1, Top Row - Initial Object Deformation, Bottom Row - Final Object Deformation. 
Figure B.8: Simulation Pr2, Top Row - Initial Object Deformation, Bottom Row - Final Object Deformation 148

Figure D.1: Static camera Simulation Frames $\{1,3,5,7,9,11\}$, respectively. 150

Figure D.2: Static camera Simulation Frames $\{13,15,17,19\}$, respectively. 151

Figure D.3: Reconfiguration through proposed method, Simulation Frames $\{1,3,5,7,9,11\}$, respectively

Figure D.4: Reconfiguration through proposed method, Simulation Frames $\{13,15,17,19\}$, respectively.

Figure D.5: Ideal camera placement, Simulation Frames $\{1,3,5,7,9,11\}$, respectively. 154

Figure D.6: Ideal camera placement, Simulation Frames $\{13,15,17,19\}$, respectively. 155

Figure E.1: Close-up view of the PLEOrb robot skin texture and color pattern. 156

Figure E.2: (a) View from left camera in arbitrary stereo pair, (b) view from right camera of arbitrary stereo pair.

Figure E.3: Matched SIFT features, left image (a), right image (b) 157

Figure E.4: Resulting triangulation point cloud, isometric view. 158

Figure E.5: Resulting triangulation point cloud, top view. 158

Figure E.6: Resulting triangulation point cloud, front view.

Figure F.1: Movie-strip for Experiment 1. 161

Figure F.2: Movie-strip for Experiment 2. 162

Figure F.3: Movie-strip for Experiment 3. 163

Figure F.4: Movie-strip for Experiment 4. 164

Figure F.5: Movie-strip for Experiment 5. 165

Figure G.1: Static camera experiment, Simulation Frames $\{1,3,5,7,9,11\}$, respectively 167

Figure G.2: Static camera experiment, Simulation Frames $\{13,15,17,19\}$, respectively. 168

Figure G.3: Reconfiguration experiments through proposed method, Simulation Frames $\{1,3,5,7,9$, $11\}$, respectively.

Figure G.4: Reconfiguration experiments through proposed method, Simulation Frames $\{13,15,17,19\}$, respectively..... 


\section{Nomenclature and Acronyms}

\section{Latin Letters}

$\mathbf{B}_{V H} \quad$ A set of initial visual hull boundary volume corner points, [8 $\left.\times k_{V}\right]$.

$\mathbf{C}_{M} \quad$ Matrix of the projected model center repeated $k$ times, $[k \times 3]$.

$C_{\text {comb }} \quad$ Total number of camera parameter combinations.

$\boldsymbol{c}_{M} \quad$ Center point of the projected model, $[1 \times 3]$.

$\boldsymbol{c}_{M}^{+} \quad$ Expected center point of the projected model, $[1 \times 3]$.

$c_{M}^{*} \quad$ Effective center point of the projected model, [1 $\left.\times 3\right]$.

$c_{o b s} \quad$ Center point of the obstacle, $[3 \times 1]$.

c Total number of cameras.

$\boldsymbol{d}_{L}, \boldsymbol{d}_{R} \quad$ Vector lengths of all vectors in $\mathbf{V}_{L}$, and $\mathbf{V}_{R}$, respectively, $[n \times 1]$.

$\boldsymbol{d}_{L}^{*}, \boldsymbol{d}_{R}^{*} \quad$ Normalized vector lengths of all vectors in $\boldsymbol{d}_{L}$, and $\boldsymbol{d}_{R}$, respectively, $[n \times 1]$.

$\boldsymbol{d}^{d} \quad$ Vector of distances from test points to the model center along $\boldsymbol{v}_{\text {test }}$.

$\boldsymbol{d}^{w} \quad$ Vector of distances from projected test points to the model center.

$d_{\text {baseline }} \quad$ Baseline distance of a stereo-camera pair.

$d_{b-\max } \quad$ Maximum allowable baseline distance.

$d_{p-\max } \quad$ Maximum allowable epipolar disparity.

$d_{\text {thresh }}^{d} \quad$ Threshold for depth distance visibility.

$d_{\text {thresh }}^{w} \quad$ Threshold for width distance visibility.

$e_{f} \quad$ Normalized relative triangulation error.

$e_{p} \quad$ Normalized prediction error.

$e_{s} \quad$ Normalized surface area error.

$e_{t} \quad$ Normalized triangulation error for surface objects.

$e_{T R} \quad$ Normalized triangulation error for solid objects. 


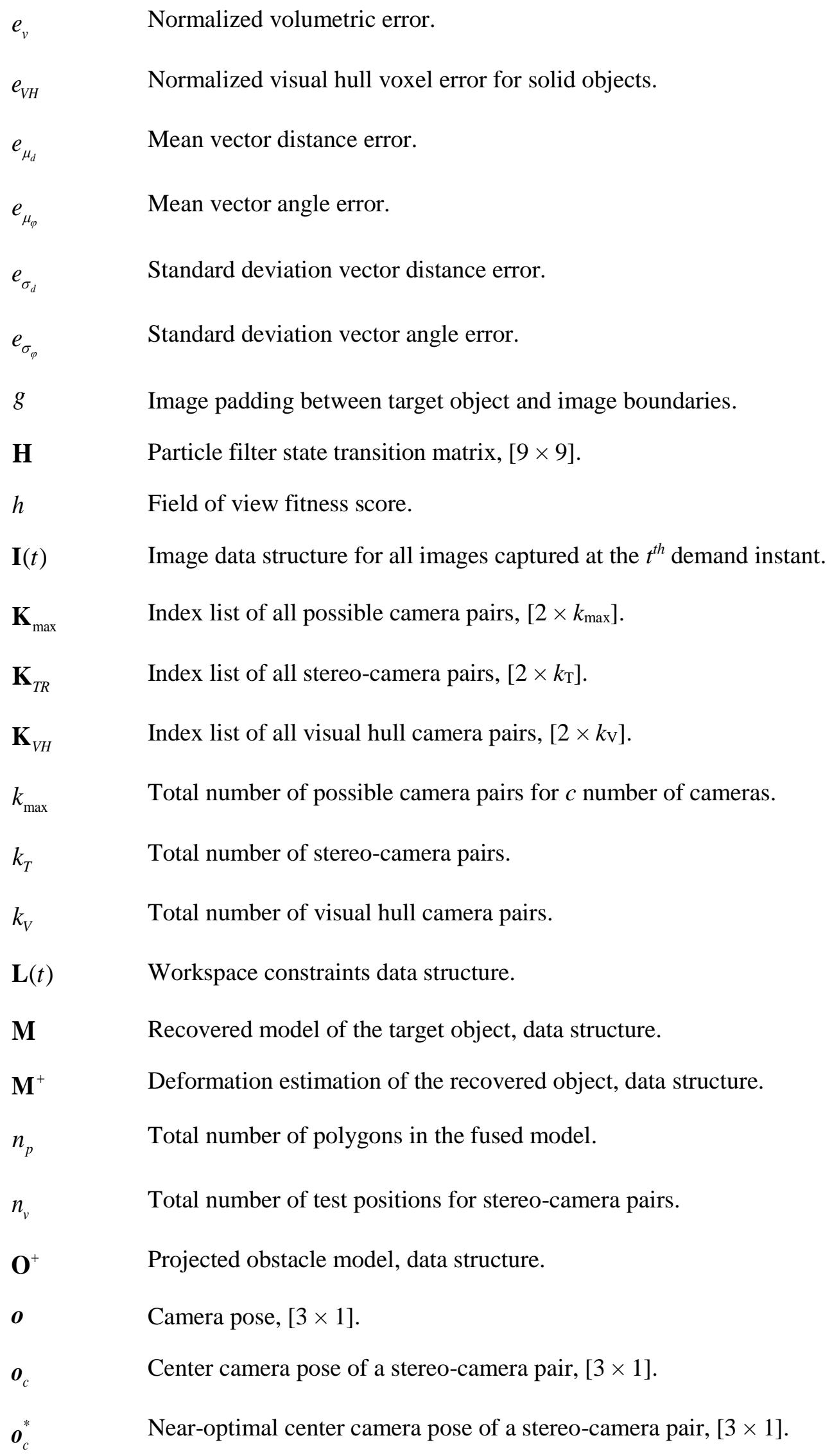




\begin{tabular}{|c|c|}
\hline $\mathbf{P}_{c}(t)$ & Camera parameters, data structure. \\
\hline$p_{\max }$ & Total number of stereo-camera positional combinations. \\
\hline$p_{\max }^{*}$ & Reduced total number of stereo-camera positional combinations. \\
\hline $\mathbf{Q}_{j}$ & Particle state matrix for the $j^{\text {th }}$ tracked point, $[9 \times q]$. \\
\hline $\mathbf{Q}_{j}^{+}$ & Projected particle state matrix for the $j^{\text {th }}$ tracked point, $[9 \times q]$. \\
\hline $\boldsymbol{q}_{i}$ & Normal vector at $i^{\text {th }}$ test point in $\mathbf{X}_{\text {test }},[3 \times 1]$ \\
\hline$q$ & Total number of particles. \\
\hline $\mathbf{R}$ & Matrix of positional combinations indices, $\left[p_{\max } \times n_{v}\right]$. \\
\hline $\mathbf{R}^{*}$ & Reduced matrix of positional combinations indices, $\left[p_{\max }^{*} \times n_{v}\right]$. \\
\hline$R(k)$ & Surface visibility score for the $k^{\text {th }}$ combination of stereo-camera poses. \\
\hline$r^{*}$ & Vector subset from $\mathbf{R}^{*}$ along a row. \\
\hline$S(t)$ & Recovered surface area of target object at the $t^{\text {th }}$ demand instant. \\
\hline$S^{+}$ & Estimated surface area. \\
\hline$S_{\text {model }}$ & Ground truth surface area of experimental target object, constant. \\
\hline$S_{T}(t)$ & Ground truth surface area of target object at the $t^{\text {th }}$ demand instant. \\
\hline $\mathbf{T}^{*}$ & Triangle mesh map for the fused object model. \\
\hline $\mathbf{T}_{T R}(k)$ & Delaunay triangulation map for the $k^{\text {th }} 3 \mathrm{D}$ triangulated point set. \\
\hline $\mathbf{T}_{V H}$ & Delaunay triangulation map applied over the visual hull voxels. \\
\hline $\mathbf{U}$ & Particle filter projection uncertainty matrix, $[9 \times q]$. \\
\hline $\mathbf{V}$ & Matrix of positional vectors, $\left[3 \times n_{v}\right]$ \\
\hline $\mathbf{V}_{\text {map }}$ & Binary visibility matrix map, $\left[n_{p} \times n_{v}\right]$. \\
\hline $\mathbf{V}_{m a p}^{*}$ & Subset of $\mathbf{V}_{\text {map }}$ for a single combination of camera poses, $\left[n_{p} \times k_{T}\right]$. \\
\hline $\mathbf{V}_{L}, \mathbf{V}_{R}$ & $\begin{array}{l}\text { Set of } n \text { vectors about a 2D corresponded features in the left, and right image, } \\
\text { respectively, }[2 \times n] \text {. }\end{array}$ \\
\hline$V^{+}$ & Estimated volume. \\
\hline$V(t)$ & Recovered volume of target object at the $t^{\text {th }}$ demand instant. \\
\hline
\end{tabular}




\begin{tabular}{|c|c|}
\hline$V_{T}(t)$ & Ground truth volume of target object at the $t^{\text {th }}$ demand instant. \\
\hline $\boldsymbol{v}_{\text {depth }}$ & Boolean vector of depth visibility tests. \\
\hline $\boldsymbol{v}_{\text {norm }}$ & Boolean vector of normal visibility tests. \\
\hline $\boldsymbol{v}_{\text {test }}$ & Test vector from $\mathbf{V},[1 \times 3]$. \\
\hline $\boldsymbol{v}_{\text {width }}$ & Boolean vector of width visibility tests. \\
\hline$v(i)$ & Grid size for the $i^{t h}$ iteration of the visual hull building process. \\
\hline$v_{\max }$ & Maximum grid size dimension for visual hull building. \\
\hline $\mathbf{W}_{j}$ & Matrix of weights for the $j^{\text {th }}$ tracked, triangulated point, $[9 \times q]$. \\
\hline $\mathbf{W}_{j}^{*}$ & Normalized matrix of weights for the $j^{t h}$ tracked, triangulated point, $[9 \times q]$. \\
\hline $\mathbf{W}_{M}$ & $\begin{array}{l}\text { Matrix of weights corresponding each visual hull surface coordinate to the triangulate } \\
\text { surface coordinates, }[n \times m] \text { for } n \text { triangulated points and } m \text { visual hull surface } \\
\text { coordinates. }\end{array}$ \\
\hline $\mathbf{X}_{\text {test }}$ & Matrix of test vertices and polygon centers from $\mathbf{M}^{+},[k \times 3]$. \\
\hline $\mathbf{X}_{\text {test }}^{*}$ & Projected $\mathbf{X}_{\text {test }}$ points, $[k \times 3]$. \\
\hline $\mathbf{X}_{T R}(k)$ & $\begin{array}{l}\text { Matrix of triangulated points positions, }[n \times 3] \text {, for } n \text { number of triangulated } 2 \mathrm{D} \text { features } \\
\text { from the } k^{\text {th }} \text { stereo-camera pair. }\end{array}$ \\
\hline$\dot{\mathbf{X}}_{T R}(t)$ & Estimated velocity of all tracked, triangulated points. \\
\hline$\ddot{\mathbf{X}}_{T R}(t)$ & Estimated acceleration of all tracked, triangulated points. \\
\hline $\mathbf{X}_{T R}^{+}$ & Projected future pose of all tracked, triangulated points. \\
\hline $\mathbf{X}_{T R^{*}}(t)$ & A subset of $\mathbf{X}_{\mathrm{TR}}(t)$ representing all tracked, triangulated points. \\
\hline $\mathbf{X}_{V H}$ & Set of all voxel-based points in the fused object model, $[3 \times \mathrm{m}]$. \\
\hline $\mathbf{X}_{V H}^{*}$ & Projected state of all voxel-based points in the fused object model, $[9 \times m]$. \\
\hline $\mathbf{X}_{V H}^{+}$ & Projected pose of all voxel-based points in the fused object model, $[3 \times m]$. \\
\hline $\mathbf{X}_{\text {voxel }}$ & Set of visual hull voxels internal to the recovered shape, $[n \times 3], n$ total internal voxels. \\
\hline $\boldsymbol{x}_{j}^{*}$ & State vector for the $j^{\text {th }}$ tracked, triangulated point, $[9 \times 1]$ \\
\hline
\end{tabular}




$\begin{array}{ll}\boldsymbol{x}_{j}^{*+} & \text { Projected state vector for the } j^{\text {th }} \text { tracked, triangulated point, }[9 \times 1] . \\ \mathbf{Y} & \text { Boolean matrix of visibility operators, }[k \times 4] . \\ \boldsymbol{y} & \text { Boolean vector of visibility operators, }[k \times 1] . \\ z_{p} & \text { Shortest distance between a projected point and true object's surface. } \\ z_{t} & \text { Shortest distance between a triangulated point and true object's surface, surface objects. } \\ z_{T R} & \quad \text { Shortest distance between a triangulated point and true object's surface, solid objects. } \\ z_{V H} & \quad \text { Shortest distance between a visual hull point and true object's surface, solid objects. }\end{array}$

\section{Greek Letters}

$\beta \quad$ Motion limiting angle for stereo-camera pair path planning.

$\beta_{v i s} \quad$ Estimated portion of surface area visible by stereo-camera pairs.

$\Gamma(t+1) \quad$ Ideal surface visibility function.

$\eta_{r} \quad$ Reduction rate of positional combinations.

$\eta_{s y s} \quad$ System visibility metric based on stereo surface visibility for simulations.

$\eta_{s y s}^{*} \quad$ System visibility metric based on stereo surface visibility for experiments.

$\theta_{\text {baseline }} \quad$ Angular separation of a stereo-camera pair's baseline.

$\theta_{b-\max } \quad$ Maximum allowable angular separation of a stereo-camera pair's baseline.

$\theta_{p-\min } \quad$ Minimum allowable angular separation between positional vectors.

$\theta_{v-\max } \quad$ Maximum tolerance range for visual hull camera pair selection.

$\kappa \quad$ User-defined image padding percentage for ESR calculations.

$\lambda_{\max } \quad$ Maximum number of visual hull building iterations.

$\mu_{d_{L}}, \mu_{d_{R}} \quad$ Mean vector lengths for the left and right cameras of a stereo pair.

$\mu_{\varphi_{L}}, \mu_{\varphi_{R}} \quad$ Mean vector angles for the left and right cameras of a stereo pair.

$\sigma_{d_{L}}, \sigma_{d_{R}} \quad$ Standard deviations of vector lengths for the left and right cameras of a stereo pair.

$\sigma_{\varphi_{L}}, \sigma_{\varphi_{R}} \quad$ Standard deviations of vector angles for the left and right cameras of a stereo pair. 


$\begin{array}{ll}\sigma_{N_{j}}^{2} & \text { Particle filter measurement variance for the } j^{\text {th }} \text { tracked, triangulated point }[9 \times 1] . \\ \sigma_{p_{j}}^{2} & \text { Particle filter noise variance for the } j^{\text {th }} \text { tracked, triangulated point }[9 \times 1] . \\ \sigma_{\text {thresh }}^{d} & \text { User-defined standard deviation threshold for depth distance visibility. } \\ \sigma_{\text {thresh }}^{w} & \text { User-defined standard deviation threshold for width distance visibility. } \\ \varphi_{i} & \text { Angular separation between a test vector and the normal of the } i^{\text {th }} \text { test point on the object. } \\ \varphi_{\max } & \text { Maximum angular separation between test vector and model point normal. } \\ \varphi_{L}, \varphi_{R} & \text { Vector angles for the vectors in } \mathbf{V}_{\mathrm{L}}, \text { and } \mathbf{V}_{\mathrm{R}}, \text { respectively, }[n \times 1] . \\ \varphi_{L}^{*}, \varphi_{R}^{*} & \text { Normalized vector angles for the vectors in } \phi_{\mathrm{L}}, \text { and } \phi_{\mathrm{R}}, \text { respectively, }[n \times 1] .\end{array}$

\section{Acronyms}

2.5D

2D

3D

API

ASIFT

CAD

CMM

DOF

EKF

EM

FOV

GLOH

HesAff-GLOH

HesAff-SIFT

IBR

\section{5-Dimensional}

2-Dimentional

3-Dimentional

Application Programming Interface

Affine Scale Invariant Feature Transform

Computer-Aided Design

Coordinate Measurement Machine

Depth of Field

Extended Kalman Filter

Expectation Maximization

Field of View

Gradient Location and Orientation Histogram

Hessian-Affine Gradient Location and Orientation Histogram

Hessian-Affine Scale Invariant Feature Transform

Image-Based Rendering 


$\begin{array}{ll}\text { KF } & \text { Kalman Filter } \\ \text { MCR } & \text { Multi-Camera Reconfiguration } \\ \text { MSER-SIFT } & \text { Maximally Stable Extremal Regions - Scale Invariant Feature Transform } \\ \text { MVP } & \text { Machine Vision Planning } \\ \text { NBV } & \text { Next Best View } \\ \text { NCC } & \text { Normalized Cross Correlation } \\ \text { ORSA } & \text { Optimized Random Sampling Algorithm } \\ \text { PCB } & \text { Printed Circuit Board } \\ \text { PCM } & \text { Principle Component Analysis } \\ \text { PTZ } & \text { Pan-Tilt-Zoom } \\ \text { RANSAC } & \text { Random Sampling Consensus } \\ \text { SFM } & \text { Structure from Motion } \\ \text { SIFT } & \text { Scale Invariant Feature Transform } \\ \text { SURF } & \text { Speeded-Up Robust Features } \\ \text { SVD } & \text { Singular Value Decomposition } \\ \text { SVM } & \text { Support Vector Machine } \\ \text { UAV } & \text { Unmanned Arial Vehicle } \\ \text { UKF } & \end{array}$




\section{Chapter 1 Introduction}

Human perception of the physical world is rooted in the ability to visually survey the surroundings and come to an understanding of physical objects. This process of visual understanding is complex, and difficult to define mathematically. For many humans, understanding and interacting with their surroundings becomes intuitive through experience and learning. Developing automated, computer-based systems capable of matching human visual perception and understanding has become a heavily researched academic topic in recent years. Such complex vision systems that mimic human's abilities must be developed on top of robust, generic methods. The necessity of such generic systems must drive research forward.

\subsection{Motivation}

Computer vision is a field of computer science and engineering that implements statistical and mathematical methods to process and understand visual data [1], [2]. The basis of any computer vision system is to break down images and recompile them into cohesive data that is understandable to humans. Examples of this span from simple hand-written digit recognition [3] to complex 3D human action recognition [4] and high resolution shape recovery [5].

Shape recovery is a high-interest topic of computer vision. The goal of shape recovery, as with any computer vision task, is to convert image data into a 3D model of a target object. The use of computer vision methods circumvents the manual process of measuring and modelling the target object using computer aided design (CAD) tools or graphical modeling software, resulting in a contact-free approach to object modelling. Shape recovery methods may be utilized to recover the shape of a priori unknown objects for completely autonomous tasks with little user-interface. Further, a reconfiguration scheme could potentially improve the accuracy of the shape recovered in dynamic workspaces. Thus, a robust, generic, and modular reconfiguration methodology for shape recovery would be applicable in many fields, and be more advantageous than currently employed ad hoc solutions. Chronologically, the progression of shape recovery methods can be categorized as contact-based, marker-based, markerless, and reconfiguration methods. Each category tends to solve a more complex generalized problem of shape recovery with applications in many fields.

Contact-based shape recovery methods are defined as those that require direct target object manipulation, namely, directly contacting the object for measurement. Direct contact methods stem from coordinate measurement machines (CMMs). CMMs may be considered as the first real shape recovery tool. These machines implement a high-precision contact probe attached to a multi-axis robot [6]-[10]. CMMs 
are comparable to markered shape recovery, or markered motion capture methodologies that replace a physical contact with engineered markers or clothes affixed to the target object, and implement a vision system to recover the shape. Herein, a distinction is made between motion capture and shape recovery. Specifically, shape recovery produces a 3D model of the target object at a desired demand instant (a discreet point in time at which a measurement is taken), while motion capture registers the deformation of a known model across multiple demand instants. Inherently, motion capture dictates the necessity for an a priori object model to correctly correlate motion between demand instants.

Marker-based shape recovery methods have been very popular in the entertainment industry [11]. Markers have been used for both facial motion capture [12] and full-body articulated model recovery [13]. Methods have been developed for real-time motion capture, allowing film-markers direct 3D visualization of an actor's performance [14]. Patterned cloth shape recovery methods are classified as a marker-based approach, with the main difference being that the known pattern of the cloth acts as a marker set [15]. Markered methods inherently improve capture conditions over CMMs, as the object does not have to be static. The main drawback of these methods is that the object model must be a priori known for 3D model association. In the case of the patterned cloth, the 3D corresponding mesh must be known. Thus, one driving motivation behind shape recovery of a priori unknown objects is the ability to produce a 3D model without direct contact, or manipulation of the object.

Markerless shape recovery methods have been developed to replace markered method for a priori known objects. A major application of such markerless methods is for articulated human motion capture [4], meshed human motion capture [16], and facial motion capture [17]-[19]. Articulated model-based methods rely on fitting the a priori known, articulated human model to the computer vision data recovered. This can be done with either a single camera [20], or multiple cameras [21]. Meshed motion capture involves an a priori scan of the model, and then shape fitting from multi-camera data [16]. Facial motion capture methods work similarly, and have been very popular for animation purposes, and focus on realtime implementation, with controlled lighting and dedicated head-worn hardware [14].

Markerless methods for a priori unknown objects have also been a widely researched topic, with solutions limited to either static objects, namely, 3D scanners, or solutions requiring extensive off-line, user-assisted processing [22]. 3D scanners have become popular in recent years with many products aimed at creating 3D models of arbitrary objects and humans [23]-[25]. The scanners move the static object or the $3 \mathrm{D}$ sensor about the object to recover the complete shape. Herein, a clarification is made that the umbrella term sensor encompasses an array of computer-vision devices including and not limited to: individual cameras, stereo-camera pairs, and range sensors such as structured-light and time-of-flight. Alternatively, workspace sensor saturation, or maximization of the number of sensors in the workspace, 
can improve shape recovery for both static and deforming objects. Workspace sensor saturation methods attempt to maximize the object's surface visibility. While these solutions offer excellent shape recovery methods for a priori unknown objects, many are incapable of either handling non-rigid objects, obstacles, or operating on-line. One approach to handle these problems is on-line camera reconfiguration.

Multi-camera reconfiguration (MCR) methods can overcome the shortfalls of static camera methods. The major benefit of MCR methods is their ability to overcome both self-occlusion and obstacle occlusion of the target object. This is even beneficial in a sensor-saturated workspace, as reconfiguration can overcome obstacle occlusion. A corollary of this benefit is that MCR methods do not require strict, highly-controlled environments. Similarly, reconfiguration can reduce the overall number of cameras necessary for shape recovery. Thus, by reducing the number of cameras in a system, the total data bandwidth required is reduced, and the overall processing costs can be reduced and re-allocated to the reconfiguration algorithm. The benefits of MCR methods could be applied to a variety of applications. It is evident that an MCR method for a priori unknown, deforming objects can act as a generic basis method for application in many fields that require shape recovery.

MCR for a priori unknown, deforming objects, has applications in the fields of medicine [26]-[28], surveillance [11], [29], [30], and entertainment [31], [32]. Surgical applications could implement an MCR method to capture the shape of deforming tissue during robot-assisted surgery. Similarly, MCR could be used for shape recovery in medical rehabilitation analysis for amputees, where a model of their body can be generated on-line. In the surveillance field, the method could be used to monitor human behavior through action recognition. MCR has a large field of applications in place of static cameras for motion capture, virtual reality immersion, and sports performance analysis. A subset of this field is the use of MCR for human-computer interfacing [33]. Finally, the MCR method could be applied toward the shape recovery of un-restrained wild animals to study their physiology and kinesiology, without capturing the animals or deploying humans to unfriendly environments such as oceans. The potential applications and current trends in computer-vision and robotics research suggest that MCR methodologies are a high-interest field of mechatronics, and entail further research to develop generic methods as basis for more complex systems.

\subsection{Thesis Statement}

The abovementioned benefits of MCR methods suggest that a methodology can be developed for the general computer-vision problem of shape recovery of a priori unknown, deforming objects. However, such a task is inherently difficult due to the implications of the problem. Specifically, the following challenges are identified that have not been well-addressed in the shape recovery framework in literature:

a. Shape recovery for a priori unknown, deformable objects 
Current methods in the literature can be grouped into a priori known objects, a priori unknown static objects, or a priori unknown objects with known identity. The first category, a priori known methods, simply uses the model of the target object to infer the current pose, and expected deformation from tracking. The second category, a priori unknown static methods, relies on the rigidity of the object to recover the shape. These methods are not applicable to deforming objects, typically, as they require to multiplex viewpoints from a single sensor. The last category of methods, for a priori unknown deforming objects, rely on a highly controlled, obstacle-free environment to recover the shape of the target object. These methods typically employ many cameras and strictly control the workspace. There does not exist a method capable of on-line shape recovery of a priori unknown, deforming objects in dynamic enviroments.

\section{b. Tracking a priori unknown objects}

Computer-vision tracking is a well-studied problem, yet, an implementation is necessary for tracking a priori unknown objects. Typically tracking methods focus on either the development of novel algorithms, the application of algorithms, or the development of specific image-based features for matching. However, a tracking method capable of robust 3D tracking for a priori unknown objects has yet to be developed.

c. Multi-camera reconfiguration for a priori unknown objects

Static, multi-camera methods have been researched for the application of motion capture using $a$ priori known models, many cameras, and highly controlled environments. The development of camera reconfiguration, and multi-camera reconfigurations has produced methods capable of shape recovery with fewer cameras in obstacle-laden environments without explicit environment control. However, none of the reconfiguration methodologies in the literature propose a generic method for reconfiguration for a priori unknown deforming objects. Typical solutions require a priori known object model for multi-camera reconfiguration, or a static object in the case that it is unknown.

The current methodologies for shape recovery are simply not capable of recovering the shape of an a priori unknown deforming object. Thus, the objective of the thesis can be broadly defined as the shape recovery of markerless, unknown deforming objects in a dynamic obstacle-laden environment through a multi-camera active-vision reconfiguration methodology.

The scope of this thesis includes the design of a generic methodology for shape recovery of a priori unknown deforming objects. Using multi-camera active-vision reconfiguration, adaptive tracking, and a model generation methodology the generic methodology would recover the shape of any solid object with 
fewer cameras than current methods in the literature with comparable accuracy. The objective of developing advanced computer-vision methodologies, such as multi-target segmentation, object recognition and learning are not within the scope of this thesis. Nonetheless, the multi-camera active-vision reconfiguration methodology proposed herein is capable of shape recovery of a priori unknown deforming objects in obstacle-laden environments.

\subsection{Literature Review}

The following review will focus on three specific topics: $i$ ) shape recovery, ii) computer-vision tracking, and iii) sensor reconfiguration. The topics are framed as the building blocks of the methodology for shape recovery of a priori unknown deforming objects. Each topic review will outline the recent developments, while the sensor reconfiguration section will also comment on the limitations of these systems in terms of their application toward shape recovery of a priori unknown deforming objects. Through this review, it will be demonstrated that a generic MCR method does not exist for a priori unknown, deforming objects.

\subsubsection{Shape Recovery}

Shape recovery methods can be classified into two categories, active methods, and passive methods [1], [6]. Active methods project a known pattern onto the object and capture an image of the object with the pattern superimposed. General methodologies include: point laser [34]-[37], laser scan line [38]-[41], and 2D pattern projection [42]-[46]. Shape-from-shading may be considered an active method when the light source pose is directly manipulated [47]. Given the global positions and orientations (poses) of the camera and the projector, the deformation of the pattern in the image is geometrically triangulated into a 3D pose. Laser-scanning and pattern projection methods tend to produce a dense depth map, at the cost of strict dependence on light conditions, and indirect object manipulation. They are, also, typically limited to surface-only recovery, and are incapable of explicit feature tracking unless an a priori model is provided. Due to their deficiencies, active methods will not be further considered, instead, passive methods will be further explored.

Passive recovery methods rely on stereo triangulation [1], [48], whereby 2D features are matched across two images and, then, located in 3D through triangulation given the known camera pose. Such methods require stereo correspondence. Multiple stereo-vision systems have been developed for highdensity shape recovery of static objects through photo-consistent feature correspondence [5], [49]-[51]. Structure-from-motion techniques reconstruct surfaces from many uncalibrated cameras [52]-[54]. One example method in [55] is capable of markerless shape and motion capture from multi-view video sequences, given complete visibility of the actor. Passive, multi-camera feature-based methods are, typically, less susceptible to lighting conditions, and do not require object manipulation [17], [56]. 
The visual hull technique was developed as a volumetric shape-recovery method utilizing passive multi-camera systems [57]. A hull is commonly defined as the 3D intersection of all back-projected object silhouettes. The accuracy of the hull depends on the number and placement of cameras [57], [58]. Recent methods have focused on the efficiency of implementation, namely, processing speed and shape accuracy [23], [24], [59], [60]. The major deficiency of visual hull methods is their inability to cope with certain concave geometries [1], [25], thusly, the visual hull only represents a bounding surface of the object. Visual hull tracking techniques also heavily rely on the existence of a priori known object model [27], [61]-[63] to ensure temporal correspondence.

Fusion techniques, generally, focus on improving the quality, and accuracy of the recovered shape. Early work in this area focused on improving the quality of recovered shape by using the advantages of both visual hulls and active range data [64]. The process results in a volumetric error ranging from 3-13\% with a minimum of 20 images required. One technique in [41] required 72 silhouette images and a minimum of 180 active scans for recovery. Alternatively, the methods in [38] and [65] use passive range data and recommend 36 images for shape recovery to produce at maximum a $10^{\circ}$ separation between images. The convex formulation presented in [66] produces accurate shapes a minimum of 24 images with passive range data. The system presented in [67] fuses active range data with silhouette information for tracking and locating both a rigid object, and manipulator tool whose pose is known to the system. Fusion methods by in [50] and [68] yield models for static and dynamic unknown objects, respectively, without tracking. The method developed in [69] fused visual hulls with stereo disparity-based data to achieve real-time recovery of concave visual hulls. Overall, these methods focus solely on static, rigid objects, and require many images for shape recovery. Furthermore, many of these methods require controlled environments, namely, well-lit, and direct object manipulation via, for example, a turntable for image acquisition. Fusion techniques have not been so far implemented for deformation prediction of unknown objects.

Generally, shape recovery methods through passive cameras exist for a priori unknown deforming objects, but they require highly controlled, obstacle-free environments. These methods are not capable of handling variations in lighting, and workspace obstacles, which makes them unfit for many real-life applications in the entertainment field, and more importantly the medical and human-computer interfacing fields. Further, these methods tend to saturate the workspace with cameras resulting in large amounts of data generated for shape recovery, which makes them very processor heavy for on-line and real-time operation. 


\subsubsection{Computer-Vision Tracking}

Tracking in a computer-vision framework is the problem of making an inference about motion from a set of acquired images [1], [70]. The problem can be approached by either tracking through detection, where the object model is strong enough for identification in each frame, or tracking through matching, where a weaker object model is combined with an object motion model [1]. Most vision-based trackers implement a tracking through matching framework as objects may change in appearance over time, degrading the performance of strong identifiers. The reader is directed to two thorough reviews of object tracking implementations and evaluations in [70] and, more recently, [71].

The tracking through matching framework can be decomposed into three steps, $i$ ) detection of features at a given demand instant and another demand instant, ii) (optional) application of motion model, and iii) correspondence of features between the demand instants. A feature is a collection of data that represents a component of an image. The tracking goal is to locate one or more features at a given demand instant, locate the same features in the consecutive demand instant, and correctly correlate them to produce a 2D motion vector between the images. Three popular algorithms for tracking include mean shift, optical flow, and key-based feature correspondence.

The mean shift algorithm is the process of data clustering that was developed initially in [72] and later generalized in [73]. The algorithm is an iterative process which shifts each data point toward the average of the data points in the neighborhood. The algorithm is classified as a mode-seeking process for locating the maxima of a density function. The algorithm is non-parametric and operates on a user-defined feature-space, allowing generalization across many applications. Due to the nature of the algorithm, one popular application has been visual tracking wherein the visual data could be parsed into a vector representation. Mean-shift tracking methods, typically, only follows steps $i$ and iii simultaneously. The algorithm is used to locate the maximum likelihood of matching data patch between two images, thus tracking the motion of the patch.

Vectorization of visual data is the process of compressing either a complete image, or image patch into a smaller dataset that represents an aspect of the image or patch. Histograms are one popular method. Color histograms have been used successfully with mean-shift to produce a tracking algorithm in [74]. Similarly, a histogram of Gaussians (HOG) features, and normalized cross-correlation has been used with mean-shift to produce a tracking algorithm in [75], and [76], respectively.

Optical flow is the estimation of a 2D motion field in an image through gradient estimation [1], [77]. The method assumes that pixel intensities are translated from one demand instant to the next. Thus, the features are the individual pixels of an image. Optical flow, like mean-shift directly identifies the 
features in both images and corresponds them simultaneously. The method assumes that surface radiance of the tracked object remains fixed between demand instants, which is a weak assumption intuitively, but has been shown to work well in practice [77]. A popular optical flow approach was proposed in [78], [79], and summarized in [80]. The tracker implementation uses an iterative coarse-to-fine approach for estimating the motion field in sets of images. The optical flow algorithm is specifically designed for motion estimation in images while mean shift is a clustering algorithm re-purposed for image tracking. Both methods are algorithms, and due to their nature do not explicitly rely on a motion model. In contrast, a feature correspondence technique in conjunction with a motion model could be implemented for 2D tracking with motion memory.

Feature correspondence tracking methods detect image features in two images, and attempt to create a correspondence between the feature sets. These methods could be applied to non-keyed feature descriptors (some of which can be labeled as classifiers [1]) such as normalized cross correlation (NCC) [5], principle component analysis (PCA) [81], and support vector machines (SVM) [82]. These methods produce a set of feature descriptors for both images, and attempt to find correspondence based on a maximum likelihood analysis of image patches. For each classifier path, the regions of maximum likelihood in the second image are corresponded, producing a map of patches between the two images.

The alterative to non-keyed features are unique-keyed feature methods. These methods assign a unique integer-based key to each detected feature in an image. The features may be quantified by various aspects. Common feature descriptors include: scale-invariant feature transform (SIFT) [83], [84], speeded up robust features [85], gradient location and orientation histogram (GLOH) [86], and histogram of Gaussians [1]. Most of these methods have led to derivative methods such as ASIFT [87], HesAff-GLOH, HesAff-Sift, and MSER-SIFT [56]. It should be noted that even the GLOH method is an extension of SIFT with the integration of PCA to further reduce the 128-integer key produced by SIFT down to 64-integers [88]. Tracking with unique-keyed features is typically more straightforward than non-keyed methods. Tracking is composed of detecting features in both image frames, and then comparing the feature matrices through a dot-product operation to locate the most similar features in both frames. To integrate a motion model step into such trackers, methods such as the Kalman filter or particle filter can help narrow the search region in images.

Filtering techniques attempt to estimate the state space of a dynamic system given a set of noisy measurements [89]. They are statistical methods that have been applied toward tracking in state-space models. The benefit of applying a filtering technique to visual tracking is the ability to produce a prediction space for the location of a feature in the consecutive demand instant. This can narrow the search region for corresponding features between demand instants and improve false-positive rejection rates. The classic 
filtering implementation was developed by R.E. Kalman in 1960 [90] and has been implemented extensively throughout literature for both 2D and 3D tracking [91]. The Kalman filter is the optimal solution for linear systems with Gaussian-distributed system and process noise [89]. Derivatives of the Kalman filter such as the extended Kalman filter (EKF), and the unscented Kalman filter (UKF) have been proposed as improvements over the original filter for non-linear systems. Particle filters, like the EKF and UKF are suboptimal filters that were developed to handle non-linear systems. They are based on a sequential Monte Carlo estimation for a set of point masses. The principle of sequential importance sampling and resampling are the basis of particle filters. Particle filters, unlike Kalman filters, are better suited for non-Gaussian noise [89], [92]. Both types of filters have been implemented in 2D and 3D computer-vision tracking tasks [91], [93]-[95].

The problem of tracking in computer-vision has been well studied in the past with many solutions proposed for both specific, and generic applications. Many methods build on previously developed algorithms from other fields of study. The number of choices for algorithms, methods, and frameworks is immense. Most of the reviewed methods focus on 2D tracking, yet 3D is also possible as an extension of 2D tracking when the corresponded features are triangulated. Similarly, filtering techniques can be applied directly to $3 \mathrm{D}$ motion instead of $2 \mathrm{D}$ image motion. Thus, in the context of this work, the tracking methodology developed should reflect previous research for multi-camera active-vision for markerless shape recovery of unknown deforming objects.

\subsubsection{Sensor Reconfiguration}

Camera reconfiguration, or in the general case, sensor reconfiguration is the process of selecting sensor positions to improve a specific computer-vision goal. This topic, in the past, has been referred to as either machine-vision-planning (MVP) [96], [97], or the next-best-view (NBV) problem [98], [99]. Three categories are herein identified under the general term sensor reconfiguration, they are: single sensor reconfiguration, multi-camera reconfiguration for 2D tasks, and multi-camera reconfiguration for 3D tasks. The topic of single sensor reconfiguration differs from the multi-camera topics as several methods have been presented in literature for reconfiguration of a single depth sensor as well a single camera. The remaining topics of multi-camera reconfiguration for $2 \mathrm{D}$ and $3 \mathrm{D}$ tasks will only review camera-based methodologies. In the remainder of this section, the terms path-planner, viewpoint selection, next best view, and reconfiguration will be used interchangeably.

\subsubsection{Single Sensor Reconfiguration}

Single sensor reconfiguration is the process of changing the pose of a computer-vision sensor such as a camera, or depth sensor to improve a quality metric for the recovered data. A popular application of single 
sensor reconfiguration is planning camera motion for animations in the film industry [100]-[102]. The topic can be subdivided into two categories: camera reconfiguration for $2 \mathrm{D}$ acquisition tasks, and sensor reconfiguration for 3D tasks. 2D tasks typically focus on camera placement to maximize a visibility metric, and avoid any workspace occlusions. This is particularly beneficial for computer graphics and animations. 3D tasks tend to reconfigure the sensor to minimize the total number of viewpoints necessary to recover the shape of a static object. The tasks can be grouped into robot-centered, image-based rendering (IBR), animation path planners, and NBV for shape recovery.

Early research into single camera reconfiguration was led by the objective of optimizing the spatial resolution of an a priori known 3D object model along an a priori known trajectory [96]. The methodology selected viewpoints of a single camera offline, such that the desired surface points of the object were kept in view, the object was kept in focus, and within the field of view of the camera. This method did not consider workspace obstacles, and was intended for application in end-effector cameras for serial robotic armatures. A similar, offline, methodology was developed in [103] with the objective of maintaining object features of interest within the camera's field of view, in focus, and correctly magnified. This method provided a characterization of the feasible camera locations and orientations of the workspace, and operated within an optimization framework for simultaneous requirement satisfaction.

The early methods were further developed to generalize the problem of single camera placement for robotic tasks. The method developed in [104] required the a priori knowledge of the sensor (camera) model, object models and trajectories and illuminator models. The objective task specifications were a collection of visibility, field of view, resolution, focus and image contrast. The output of the generalized MVP was the camera's pose, optical settings and illuminator pose. The methodology calculated camera trajectories offline. A follow up survey was then conducted in [97] which reviewed single camera MVP techniques in terms of their inputs, objective functions and constraints.

An alternative approach to the single camera path planner was developed for viewpoint planning an a priori known robot task [105]. The method analyzed the geometric models of the environment, camera, and robot, and integrated swept volumes with a temporal interval search technique to plan valid viewpoints of the robot task at varying temporal intervals. The viewpoints were achieved by mounting the camera on a separate robot manipulator. An unconstrained, uncalibrated, single camera reconfiguration method was developed in [106]. The reconfiguration method estimated 3D point location of the target object and planed the camera path to maintain object visibility. A path planner was developed in [107] to generate the optimal camera path for the task of inspecting printed circuit boards (PCBs). The method minimized camera motion by locating the shortest paths from points of interests given the a priori known PCB design. A followerrobot was designed in [108] that affixed a pan-tilt camera to the robot chassis. The camera would 
reconfigure on two axes to center a tracked target in its view, and the robot would then rotate and translate to maximize the projection of the object in the field of view, resulting in a following motion. This reconfiguration was purely on-line and based on converting 2D tracking to 3D motion. Similarly, a 3D exploration method employed the NBV approach to map a static environment about a mobile robot in [109]. The method implemented a receding horizon scheme wherein an on-line computer random tree search located the best branch through which the maximum amount of unmapped space could be explored.

Camera reconfiguration was also applied to the fields of image-based rendering (IBR), where 2D images where rendered from an a priori known 3D scene. A method developed in [110] used 3D polygon input models to determine the viewpoints necessary to maximize the polygon visibility through a viewpoint entropy metric. The metric quantified the amount of information seen from a given viewpoint. An alternative quality metric using mesh saliency was proposed in [111] for the application of viewpoint selection given the objective of maximizing scene information. The metric implemented a center-surround operator on Gaussian-weighed mean curvatures, and produced more pleasing results in the selected viewpoints. An extension of the salient features method was developed in [112] for viewpoint selection of time-varying volumes for cinematic applications. The method's objective was to maximize the amount of information recovered from 2D imaging of the scene. Another approach to the viewpoint selection problem using volumetric representations of the target object in 3D was developed in [113]. The method attempted to maximize the information perceived in viewpoints by characterizing the surface with features based on the topological transitions of isosurfaces.

Cinematography-based camera reconfiguration methods are similar to IBR in that the quantity of information conveyed through an image is important, but importance is also placed on the path the camera travels, as it must be collision free, and to an extent, it must consider the artistic nature of camera motion. Several papers provide excellent reviews on past research on camera reconfiguration and path planning for computer-graphic applications of video games and animations in [100], [102]. A numerical method for search-space viewpoint pruning was developed with applications in cinematography [114]. The method avoided collisions with 3D modelled objects and accounted for the various types of cinematic shots commonly employed in films.

An efficient method for camera path planning was developed for human motion in animation [115]. The path planning objective was based on producing informative viewpoints revealing significant character motions while maintaining smooth camera motion. To improve efficiency over computationally expensive methods that search over the entire workspace, the method proposed heuristically generated the camera path through a greedy tree traversal approach. Further, the method allowed for user-interactive camera control. An on-line camera path planner was developed for the animation-based motion capture of a human 
character in [116]. In place of explicit motion of the cameras in the workspace, a set of candidate cameras were deployed with fixed location in space. The algorithm, then, in an on-line fashion, chose the viewpoint that best conveyed the human character's motion. This approach could overcome severe occlusions and scenes with multiple characters. Another camera path planner was developed with the objective of reducing of motion sickness by imposing a $C^{2}$ smoothness constraint on camera motion in [101]. The method implemented Pythagorean hodograph for path planning under the assumption that all viewpoints along a curve are achievable by the camera. Interpolation algorithms were used to avoid obstacles in the workspace and eliminate collisions with the cameras. The viewpoints were selected to maximize entropy.

The literature highlights the large interest placed in single camera reconfiguration for robotic tasks, IBR, and cinematography. Almost all the above-mentioned methods require a priori knowledge of the either the target object and the environment or both to accurately reposition the camera for a $2 \mathrm{D}$ capture task. In contrast, certain single camera and single sensor reconfiguration methods exist for the 3D shape recovery of objects. These methods tend to implement active sensors, specifically, a depth sensor in place of an individual camera, and are limited to static object shape recovery. The principle behind 3D shape recovery from a single, reconfigurable sensor is similar to 3D scanning technique where a rigid object is placed on a turn-table with a fixed sensor, and rotated in angular increments while the sensor gathers 3D data, and then compiles it into a single model. The main difference for single sensor reconfiguration is that the reconfiguration is either planned to minimize the total number of views necessary for model generation, or it is planned to explore to whole 3D shape without a priori knowledge of the object or its identity.

A method for planning the NBV for 3D surface acquisition was proposed in [98] using a single range scanner. The viewpoints were chosen based on a void-model analysis to determine where missing components of the recovered shape exist by searching along the edge of the recovery data. The method did not require a priori knowledge of the object, but the object must be static. A similar method for a 2.5D range sensor was developed in [117]. The method chose the viewpoint through an optimization process based on the objective function that measured the quantity of unknown data in each potential viewpoint. Potential viewpoints were automatically generated in a uniform distribution about the object (in a turn-table style), or calculated from an occupancy grid model. The model was built in voxel representation and shape recovery was deemed complete once the number of voxels ceases to increase.

A camera-laser scanning sensor was used for the NBV method developed in [118]. The objective of the viewpoint selection algorithm was to guarantee uniform object resolution. To fulfill the objective in the view planning framework, an optimization framework was implemented to minimize the loss of information based on surface entropy. The method was fixed to a circumferential reconfiguration space, and limited to rigid objects. Another approach to the NBV problem was to plan the sensor path according 
to the recovered trend surface at each sampling step [119]. The recovered surface patch was analyzed, and the region with the lowest surface order was selected as the best direction for further exploration. The method automatically chose the best direction without user-assistance, and was capable of shape recovery of a priori unknown static objects.

One algorithm for the single sensor NBV positioning considered the error from uncertainty in sensor positioning [120], [121]. The proposed algorithm implemented a search-based paradigm which generated candidate viewpoints for evaluation. Once the best the viewpoint was chosen, a secondary process re-evaluates the viewpoint quality relative to their neighbors to ensure the chosen viewpoint was in a good neighborhood. The recovered shape was represented as a voxel-based object. Another volume-based approach used volumetric exploration [122]. The data was presented as a visual trial for the systematic revelation and illustration of the object volume. Potential fields were made through projection maps and used to plan the sensor path. The method required user-assisted key-feature selection for the next best viewpoint estimation.

Visibility analysis and ray-tracing were used to determine the NBV for a single 3D scanner with minimal a priori information of the target object in [123]. Surface patches of the object were generated in real time as the object was rotated on a turntable. The method consisted of two steps: target region identification and best viewpoint identification. The output model used a polygon mesh representation.

Single sensor reconfiguration has major applications in robotic exploration, quality control, cinematography, and medical imaging. The research into intuitive reconfiguration has improved the efficiency of the tasks completed, and led to on-line and real time methodologies. However, there exist several limitations in the use of single sensors for both visual tracking tasks, and shape recovery tasks. Firstly, a single sensor is limited to the field of view of the sensor, thus limiting the scene coverage of the system in comparison to a multi-sensor system. Secondly, single sensors cannot accurately recover the shape of deformable objects due to the limited single viewpoint. Therefore, the task of shape recovery of $a$ priori unknown, deforming objects must be handled by a set of multiple sensors, or more specifically, a set of multiple cameras. Multi-camera reconfiguration systems and methods have been developed for both 2D visual servoing tasks, and for 3D recovery and servoing tasks.

\subsubsection{Multi-Camera Reconfiguration for 2D Tasks}

Multi-camera reconfiguration for 2D tasks is, typically, the process of reconfiguring the pan-tilt-zoom (PTZ) parameters of individual cameras in a network [124]. These PTZ networks are commonplace across applications including building surveillance, public area monitoring, traffic monitoring and crowd flow analysis. Many networks are controlled by a human operator to avoid the data processing requirements for 
automated reconfiguration control. One option explored to circumvent an on-line control algorithm was to analyze the workspace a priori, and then optimize for camera placement to maximize possible visibility [125]-[127]. Alternatively, cameras may be controlled on-line to reconfigure for objectives such as target resolution, workspace coverage and visibility, and target tracking, [124], [128].

PTZ network reconfiguration for target resolution modifies camera parameters to maximize the capture resolution of one or more targets by the system. High resolution imaging of objects of interest in a camera network is beneficial in surveillance applications for biometric identification. A PTZ camera assignment and handoff methodology to maximize target resolution was approached as a planning problem in [129]-[131]. The methodology applied wide field of view, static, support cameras to provide target state information. The PTZ planning problem then optimized the reconfiguration parameters of the active cameras to maximize the resolution of a desired target in the workspace, and planed the handoff activities between active cameras to ensure smooth data capture. A Bayesian framework was applied in [132], [133] to mitigate the risk of losing a target in track when reconfiguring a PTZ network for high resolution acquisition. The framework was built around a distributed approach for camera control. A Kalman filter was implemented for tracking, providing an estimate of future target location, and, in turn, allowing the framework to proactively update camera parameters. An elderly case-specific for fall detection method was developed to operate on the compressed image data-stream in [134]. The method detected anomalies in the video stream, specifically - a fall, and redirected network resources through reconfiguration to improve resolution of the event. The event detection was handled on the compressed video stream through an event descriptor based on the entropy of the motion detected. Once a fall event was detected, the cameras attempted to center the event in the frame, and maximize the resolution of the target through zooming.

Coverage-based PTZ reconfiguration could be considered the opposite objective of resolutionbased reconfiguration, as coverage tends to maximize workspace visibility simultaneously instead of narrowing visibility to a single target or event. An expectation maximization (EM) technique was applied in [135] to improve workspace visibility and coverage to improve detection of a desired activity. A spherical activity mapping technique was applied to simplify the reconfiguration optimization space for EM application. The EM technique was further improved for application in occluded environments by modifying the spherical activity maps [136]. Direct modification of these maps resulted in explicit handling of occlusions of the workspace. Limited examples exist for the coverage-based reconfiguration of PTZ networks, as generally coverage is an objective for static cameras and is optimized once for initial camera deployment. Generally, PTZ network reconfiguration applications have focused on the single and multiple target tracking problem. 
Camera network reconfiguration for tracking is a popular research topic due to its applicability across many surveillance tasks [124]. A real time tracking method was applied to track human motion in a PTZ network and automatically reconfigure cameras in [137]. The cameras were calibrated, used meanshift tracking in $2 \mathrm{D}$, and basic triangulation to estimate the human's position in the workspace prior to reconfiguration. A decentralized approach to PTZ reconfiguration was developed for target tracking in [138], [139]. The method applied a game-theory approach to reconfigure the cameras for single and multitarget tracking. Target tracking and cross camera association was the goal of the PTZ reconfiguration method proposed in [140]. The objective was to map a target's feet to their face in two different views to improve tracking across the network and simultaneously the visibility.

A partially observable Markov decision process-based approach was used for target tracking through a PTZ network in an uncertain, partially observable environment in [141], [142]. The method monitored the targets kinematic state to track under both visible, and non-visible conditions. At the same time, the camera network was reconfigured to improve tracking data of the targets and maximize the total number of target visible in the network. An entropy analysis method was proposed to track multiple targets in the workspace and simultaneously improve workspace coverage in [143]. The visual entropy measure drove the global sensor assignment scheme for coverage mean while the local image motion field disorder drove the target tracking tasks in the network. An on-line learning approach was developed in [144] to automatically learn reconfiguration behavior tasks for target tracking. The method implemented a reasoning logic for handling new events that trained the system to reconfigure for tracking tasks.

PTZ networks are popular in both research and industry due to their commonplace applications. Reconfiguration allows for improved PTZ surveillance for high resolution target biometric identification, and event detection in larger environments. Through reconfiguration, an individual or team of human controllers could be replaced with automated algorithms that may outperform humans in terms of detection and reaction speeds as multiple data-streams maybe processed at once. Several reconfiguration methodologies are being developed for 2D tasks through the coordination of unmanned aerial vehicles (UAVs) for target tracking, and workspace coverage as [145]-[147]. The main deficiency of these networks is their inability to process $3 \mathrm{D}$ data directly, which limits them to $2 \mathrm{D}$ image processing, and at best $2.5 \mathrm{D}$ scene inference. Thus, to develop a multi-camera shape recovery methodology, other research methods must be explored, namely, multi-camera reconfiguration methods for 3D data acquisition.

\subsubsection{Multi-Camera Reconfiguration for 3D Tasks}

Multi-camera reconfiguration (MCR) for 3D consists of modifying camera parameters to recover 3D target data. The major benefit of MCR for 3D tasks is the ability to either track or recover the shape of target 
objects in the presence of obstacles and occlusions. 3D tracking allows the systems to proactively reconfigure to maximize visibility objectives. Shape recovery tasks also benefit from 3D tracking, as systems can also reconfigure to produce the necessary viewpoints for shape recovery. In contrast, single sensor methods provide only a limited field of view and cannot produce multiple viewpoint simultaneously for shape recovery, while PTZ networks typically cannot overcome dynamic occlusion without saturating the workspace with cameras.

Early systems focused on the dynamic dispatching of cameras to track the 3D trajectories of single or multiple target objects [148], [149]. These methods were developed for real time camera reconfiguration to achieve optimal data acquisition given the a priori information about target trajectories and initial camera poses. The camera poses were optimized on-line to maximize the effectiveness of the sensing system through a non-linear, direct search method in parallel to a simulation of the system's performance. An experimental implementation of dynamic dispatching method was presented in [150]. The implementation integrated a static overhead camera to monitor the trajectory of the object, and four mobile cameras. The target's trajectory was a priori unknown to the system, and the mobile cameras were independently and optimally positioned on-line to improve the estimate of the target object's pose. The camera data was fused to produce a single 3D coordinate measurement of the target's location in the workspace. A Kalman filter was used to estimate the target's state. The complete experimental implementation was developed in a modular fashion, allowing scalability and easy modifications.

The experimental methodology was later improved for implementation in a cluttered environment [151]. The updated methodology's objective was to maximize the visibility of the target object by reconfiguring the cameras while avoiding obstacle occlusions in the workspace. Optimal viewpoints were chosen through evaluation of the visibility metric at discreet positions. Experimental results showed the reconfiguration scheme capable of location multiple desired targets from a dynamic, cluttered workspace. A final iteration of this reconfiguration scheme was developed for temporal sensor scheduling, assigning time-based tasks to various cameras to ensure the desired target objects are visible at specific demand instants [152]. The methods relied on modelling the target object and obstacles as simple geometric primitives which limited the 3D recovery to location instead of shape. Simulated and experimental results validated the methods' ability to track desired targets in the workspace and hand-off visual servoing tasks between cameras during occluding regions.

A MCR method was developed for 3D tracking and recovery of 3D articulated shape of humans in [153]. The mobile, stereo-camera robot was developed to estimate the 3D articulated shape of humans the robot encountered. Multiple mobile stereo camera units were used to reconstruct the 3D shape offline. One advantage of this method was the use of calibration-free cameras while relying on the factorization method 
[154] for shape acquisition. The final output of the system was a motion capture sequence of the human actor's actions. A similar approach was used specifically for the articulated motion capture in sporting events [155]. The method did not require camera calibration, and implemented a centralized processing unit for data reconstruction from multiple cameras. In both works, the camera motions were user controlled to ensure valid viewpoints were chosen for recovery. This is a major system deficiency when compared with other automated 3D tracking and motion capture techniques.

A combined static and reconfigurable multi-camera system was presented to capture 3D video in [156]. Individual cameras were automatically assigned to monitor surface patches of the target object during the object's motion. This automatic assignment of the cameras was based on analyzing the coarse model generated during run-time. The reconfigurable cameras were limited to PTZ motion, and were assigned viewpoints on-line via a visibility objective function. The object model was built using a visual hull. The method required explicit control of the workspace, and did not allow for obstacle occlusion.

More recently, a method was presented for the articulated shape recovery and action recognition of a human actor with an a priori known model [4], [29]. The method was developed around an agent-based approach which solved the reconfiguration problem for 3D shape recovery and action recognition through a multi-camera system. The agent-based approach coordinated the tasks between six individual agents, namely, sensor, referee, central planning, prediction, action recognition, and form recovery. Each agent was responsible for select tasks and ensemble, the system produced the shape and recognized the actions of a human model. The cameras were reconfigured on-line based on the predicted articulated pose of the object through Kalman filter joint tracking fused with a visibility analysis of the workspace. The objective function searched for viewpoints to maximize the object's visibility given dynamic workspace obstacles. A real time implementation of the methodology was presented in [157]. The major deficiency of this system was the necessity of the a priori known, articulated object model. The articulated model limited the applications of the system, and meant that surface deformations of the object could not be recovered.

An improved MCR method for the motion capture of deformable objects was proposed in [30], [158]. The method required an a priori known polygon object model with distinct object markers for correct detection and association. A Kalman filter was used with constant-velocity dynamics to track the triangulated marker positions. The cameras were reconfigured on-line by maximizing the markers' visibility in the cluttered workspace, to ensure correct triangulation. The simulation and experimental results indicated low recovery error and improved performance over static and limited motion reconfiguration methods. The major drawback of this method was the necessity for an a priori known model with engineered surface markers. 
The MCR methods in literature demonstrate the clear advantage of camera reconfiguration for 3D shape acquisition tasks due to their ability to overcome cluttered, dynamic environments without saturating the workspace with cameras, or more generally, sensors. Further, it is evident that positional reconfiguration allows for improved viewpoints over pan-tilt only, as pan-tilt limits cameras to a spherical viewing field about the center of the camera, while positional reconfiguration changes the viewing field completely. Unfortunately, there does not exist a single, generic, modular method for the 3D shape recovery of a priori unknown, deforming objects.

\subsection{Thesis Contributions}

The literature review presented above covered many topics that are pertinent to the 3D shape recovery of $a$ priori unknown, deforming objects. These topics include shape recovery methods that deal with model generation, tracking methods, and most importantly reconfiguration methodologies. The review of shape recovery methods illustrates a sample of available approaches to render a 3D shape from visual data. Similarly, the review of tracking methods demonstrates the thorough research existing in literature that is available when designing new methods and systems. In contrast, past reconfiguration methods do not provide a single generic method for shape recovery of a priori unknown deforming objects. Every method reviewed in the literature depends, to an extent, on one of the following: a priori known model, a priori known model identity, a highly control environment, static objects, and workspace sensor saturation. Thus, it is clear, that a shape recovery method for a priori unknown deforming objects does not exit.

To develop such a method through multi-camera active-vision reconfiguration, three general parts are proposed: model generation, tracking and prediction, and a reconfiguration schema. Thus, the contributions of this work are threefold: firstly, a methodology will be established for the recovery of the 3D shape of a target object, then, a robust tracking and prediction framework will be built to support deformation prediction, and finally, the reconfiguration scheme will be developed to ensure overall improvements in shape recovery through a designated objective metric. The three parts will then be integrated into a single, modular, generic methodology for the shape recovery of a priori unknown deforming objects.

The first contribution of shape recovery is based on a fusion of 3D data methods, specifically, stereo triangulation, and the visual hull process. The proposed methodology can produce a complete, $3 \mathrm{D}$, shape estimate of the target object, specifically with a smaller number of cameras than static-camera networks, and other fusion techniques. The model output of the fusion technique is a trackable, solid object. The object's surface is modelled with an uncertainty estimate that dictates the likelihood that the surface of the 
object recovered exists in the location it was recovered. This is a necessary condition for poor camera placement when the object's surface visibility is not complete.

The second contribution is the development of an adaptive particle filtering framework for tracking the recovered object model and producing a deformation estimation. The filtering framework is modular in the sense that the motion model can be interchanged depending on any a priori known motion constraints. The filter is adaptive in its ability to dynamically allocate the total number of tracked features and modify the precision of the tracking on-line. The use of a particle filter overcomes certain deficiencies of optimal Gaussian filters that exist in computer-vision applications.

The third contribution is the reconfiguration scheme responsible for the objective metric selection, reconfiguration space generation, metric analysis, and camera path planning. The objective metric is established in a logical manner based on an analog to a human's perception of an unknown deforming object. The reconfiguration space is generated to account for all feasible camera positioning. The metric analysis evaluates the objective function at all achievable camera positions, and selects the near-optimal. The camera's paths are then planned toward the near-optimal positions based on imposed motion limitations in the system.

Finally, all three parts are fused into a single, modular system that is capable of shape recovery of a priori unknown deforming objects. The system is capable of accurate shape recovery with fewer cameras than certain static methods, and produces a complete 3D mesh model of the object. The system also outperforms static camera methods and approaches the performance of an ideal reconfiguration methodology.

\subsection{Thesis Outline}

The remainder of the thesis is organized as follows. Chapter 2 mathematically develops and outlines the problem of a multi-camera active-vision reconfiguration method for a priori unknown, deforming objects. Chapters 3-5 present the development of each component of the methodology in terms of the complete system. Chapter 6 presents the simulation framework for testing the methodology and includes simulation results with comparisons of the proposed methodology with static camera networks, and an ideal camera reconfiguration method. Chapter 7 presents an experimental platform framework and experimental results for testing the methodology along with comparisons to static camera networks. Chapter 8 summarizes the methodology and findings of the thesis, and offers remarks and recommendations on future works. 


\section{Chapter 2 \\ Problem Definition}

Past research has focused on individual computer-vision problems such as static shape recovery of both $a$ priori known and unknown objects, and shape recovery of a priori known deforming object. Several attempts have been made toward shape recovery of a priori unknown deforming objects that were limited to highly constrained workspaces with a priori known model identities, namely, articulated human models. The a priori known identities of the models enabled the methods to accurately estimate shape without a complete a priori known model. Thus, the problem addressed by this dissertation is the general approach to shape recovery of a priori unknown deforming object through a multi-camera active-vision method in unconstrained environments. The generalization of this problem leads to a new set of challenges that have not yet been addressed in literature.

This chapter is divided into two sections. The first section generalizes the objectives of shape recovery methods in computer vision. The second section applies the generalization within the framework of this dissertation and presents the individual challenges that must be addressed.

\subsection{Generalization of the Shape Recovery Objectives}

Shape recovery is the process of generating a 3D model of a target object through a computer-vision method. Target objects for shape recovery are classified into two different categories: homogenous solids, and single-sided surfaces. Examples of single-sided surface-based target objects include geographical topologies, or human facial capture [17]. Solid-based models typically capture a complete human-body [16]. Both types of objects' surfaces are assumed to be singular, and continuous such that they may be sampled with infinite precision. The objectives of shape recovery are defined as accuracy and model completeness. These objectives must be generalized for both types of object categories.

\subsubsection{Objective Definition}

The first objective of shape recovery, as with any measurement method, is to maximize the accuracy of the measurement within a global reference frame. Maximizing the accuracy of the 3D model is the equivalent to minimizing the Euclidean distance error between the sampled surface coordinates and the object's surface in the sampling neighborhood:

$$
\min (|x-p|)
$$


under the condition:

$$
\frac{x-p}{|x-p|} \bullet n_{p}=1
$$

where $x,[3 \times 1]$, is a sampled surface coordinate from the model, and $p,[3 \times 1]$, is the nearest surface coordinate on the true object. The Euclidean distance error is the vector length between $x$ and $p$. The condition in Equation (2.2) ensures that $p$ is the closest true surface coordinate to $x$ by enforcing a normal constraint, such that the vector between $x$ and $p$ is parallel to the surface normal at $p$ defined by $n_{p},[3 \times 1]$.

The second objective of shape recovery approach is to maximize the completeness of the model. Model completeness is defined by the type of target objects. Surface-based objects' completeness would be defined by the total surface area recovered as bound by certain features such as the jaw line, hair or similar [14], [17]. In contrast, solid-based objects would define completeness as the volume and surface area recovered.

The objective of model completeness can thusly be defined as the minimization of the squared error between the recovered volume and true volume, and the squared error between the recovered surface area and true surface area:

$$
\begin{aligned}
& \min \left(\left(V(t)-V_{T}(t)\right)^{2}\right), \\
& \min \left(\left(S(t)-S_{T}(t)\right)^{2}\right),
\end{aligned}
$$

where $V$ and $S$ are the total volume and surface area of the recovered model, and $V_{T}$ and $S_{T}$ are the true volume and surface areas of the target object, all taken at a particular demand instant, $t$. Solid-based objects' completeness is governed by both Equations (2.3) and (2.4), while surface-based objects' completeness is only governed by Equation (2.4). By themselves, Equations (2.3) and (2.4) cannot define the shape recovery accuracy as they do not quantify the relative position of the recovered model compared to the true object in a global coordinate frame. Therefore, the completeness objective of shape recovery must be coupled with an accuracy measurement to ensure surface sampling accuracy within a fixed reference frame.

The completeness objective for a solid-based object is visualized in Figure 2.1, where the left model is incomplete due to its coarseness, while the right model is more complete. Incomplete models result in both missing volume data and surface data. 

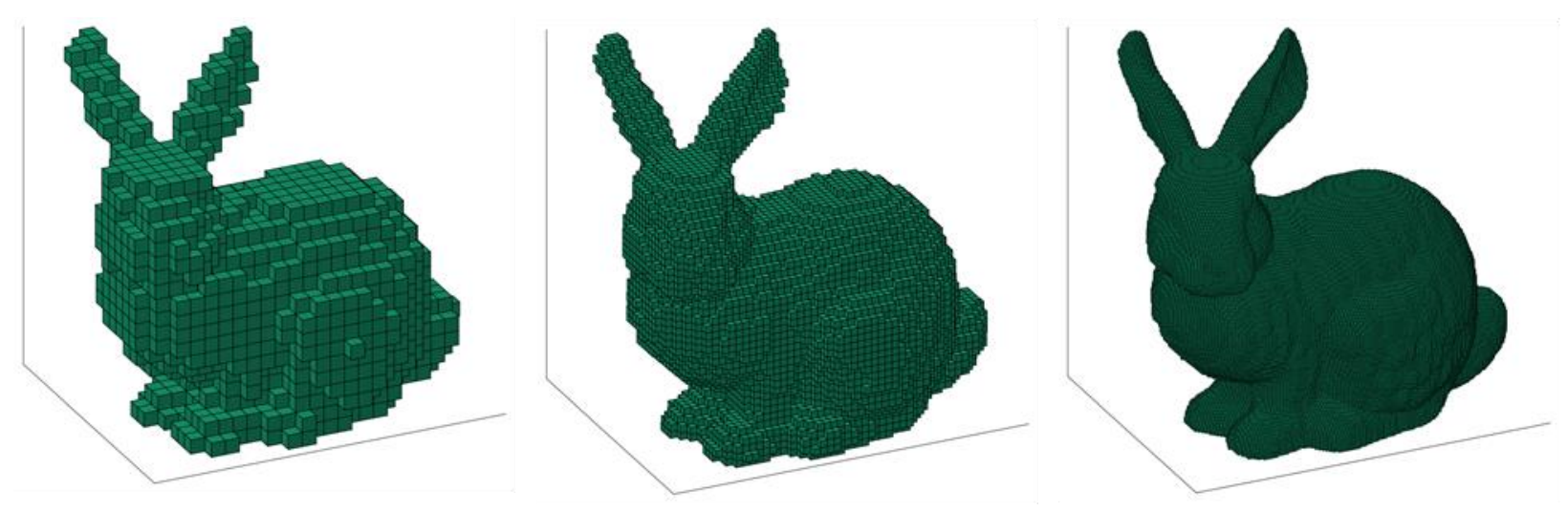

Figure 2.1: Volumetric model completeness, left: low, middle: medium, right: high.

\subsubsection{Objective Generalization}

The accuracy objective of shape recovery is based on sampled surface coordinates. The completeness objective is based on surface area and volume. A generalized approach to shape recovery in terms of both objectives cannot directly include volume, as surface-based objects lack an explicitly defined volume. Therefore, the generalized completeness objective must be re-framed in terms of surface area to remove ambiguity in cases where volumetric measurements are not feasible.

Generalizing the shape recovery objectives in terms of an object's surface requires a reformulation of the volumetric completeness objective for solid-based objects. This can be achieved by separating the volumetric completeness into a four-step process, namely, maximizing the surface area visibility, recovering surface patches, hole-filling missing patch data, and estimating the volume from the recovered shape. Intuitively, maximizing the surface area visibility will result in the largest recovered surface area which would minimize the amount of hole-filling necessary to produce a solid model. Many robust holefilling algorithms are available in literature [159]-[161]. The solid model would produce the best approximation to the true object. Therefore, by simply maximizing the surface area visibility, the volumetric error can be indirectly reduced and addressed as a surface-based objective. Thus, the shape recovery objectives of accuracy and completeness for both object categories can be generalized in terms of the objects surface. The accuracy objective correlates to maximizing the surface sampling accuracy, and the completeness objective correlates to maximizing surface area visibility.

The objectives of shape recovery can be further generalized as the task of uniformly sampling the 2D unwrapped surface or feature space. This approach is based on model learning methodologies [162]. UV mapping is a typical method for projecting the 3D model's surface into 2D space, Figure 2.2, wherein each point or sample on the feature space represents a finite surface patch on the object. Therefore, by 
uniformly sampling the 2D feature space with increasing density, the recovery method maximizes the total unique surface area recovered, Figure 2.3. The uniform sampling approach is analogous to maximizing the surface area visibility of the target object, thus, the higher density uniform sampling of the feature space would produce a complete model. Conversely, a non-uniform sampled feature space would result in poor model completeness, Figure 2.4.

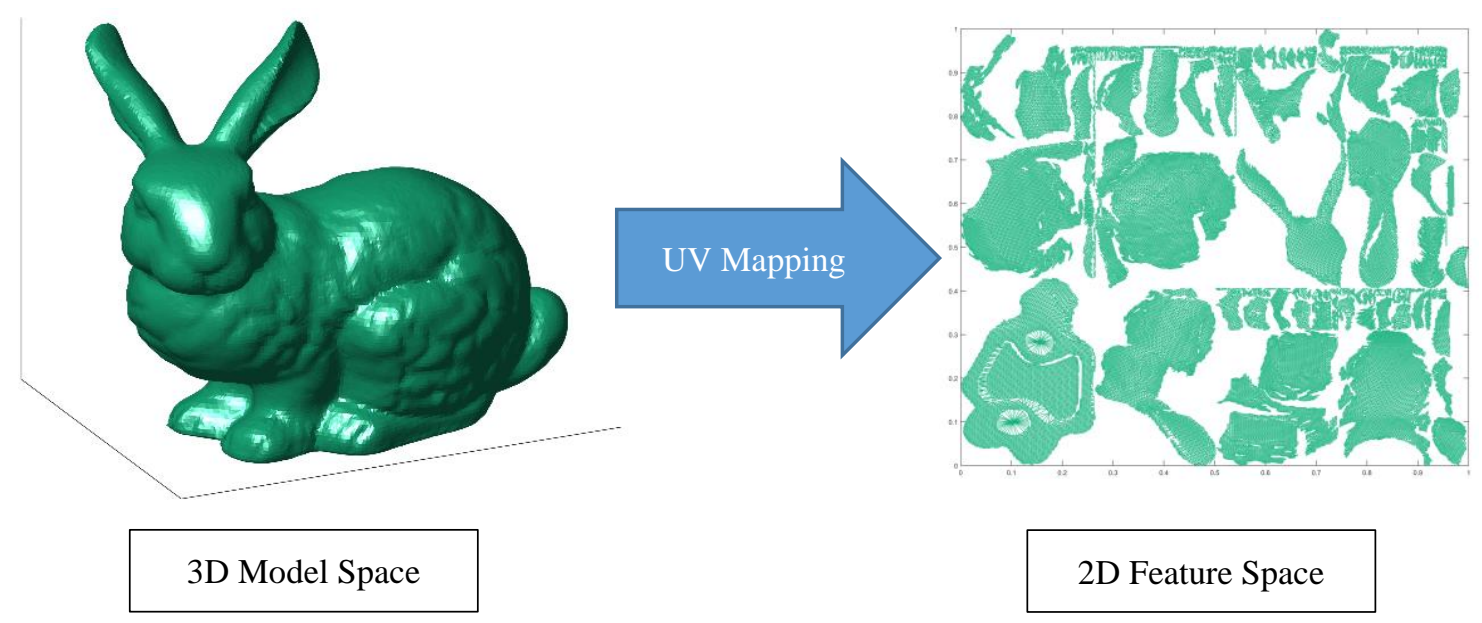

Figure 2.2: Projection of the 3D manifold into 2D feature space.

The objectives of shape recovery are now both generalized in terms of the object's surface, and framed within a preliminary visibility optimization in terms of the object's feature space. The accuracy objective states that the sampled surface coordinates of the object must minimize the Euclidean error between sampled coordinate and true object's surface. The completeness objective states that the recovery must maximize the surface area visibility of the object. Together, these objectives generalize the shape recovery objective for both surface-based and volume-based target objects.

\subsection{Application of the Generalized Shape Recovery Objectives}

The generalized approach to the shape recovery objectives can now be applied towards the problem addressed by this dissertation, namely, the recovery of a priori unknown deforming objects in dynamic workspaces through a multi-camera active-vision system. A multi-camera active-vision system is one capable of on-line camera parameter modification. A priori unknown objects are defined as target objects whose models and identities are both unknown to the system. Therefore, it is not possible to measure the accuracy of a recovery method, nor deterministically map the feature space. Herein lies the challenge this dissertation will address. 

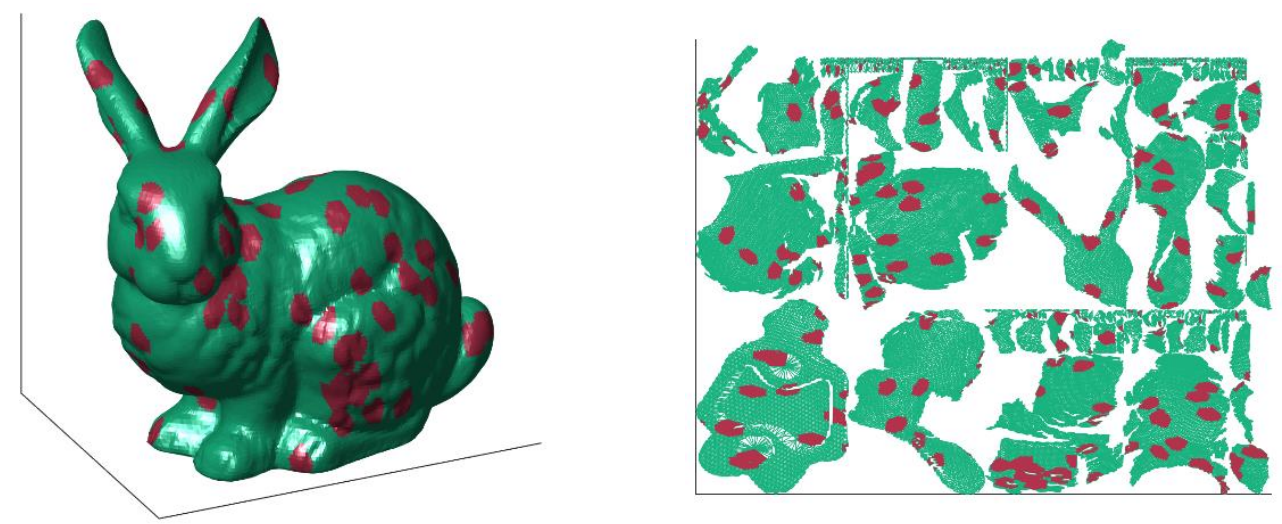

a) Low density sampling
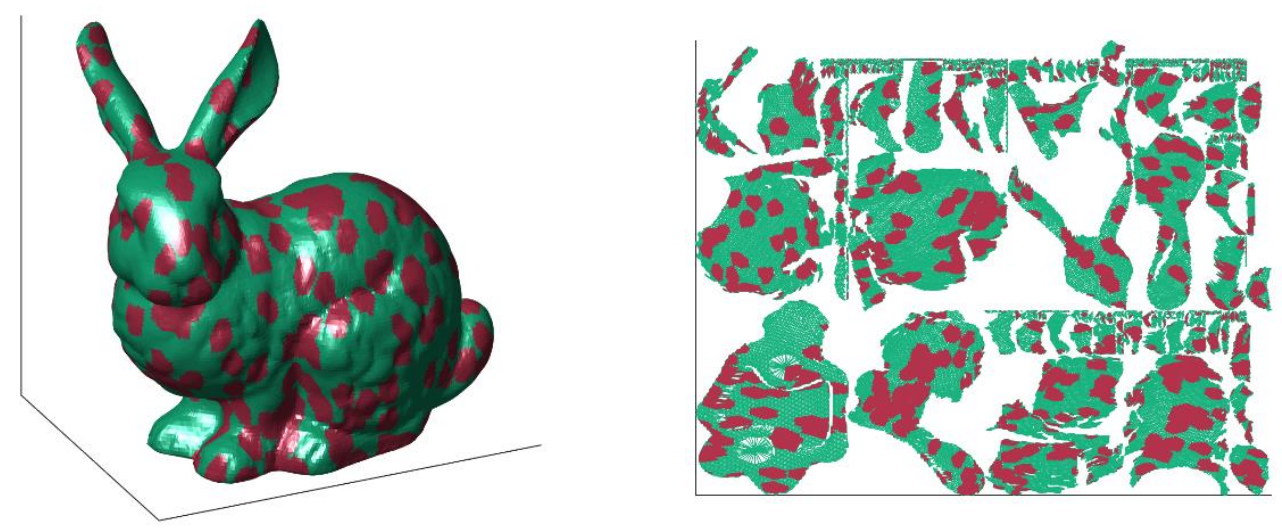

b) Medium density sampling
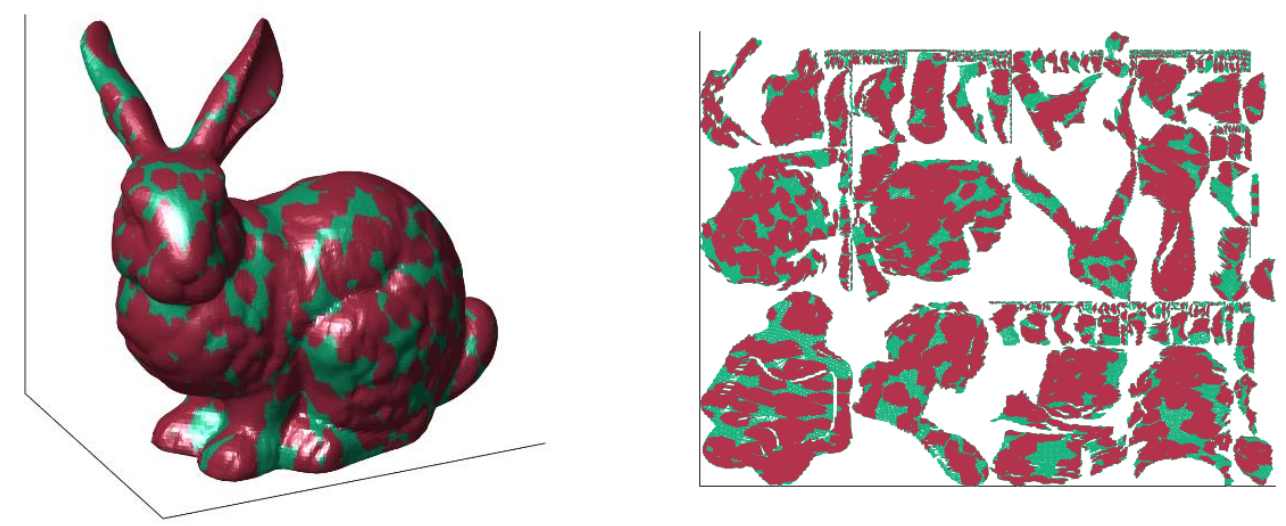

c) High density sampling

Figure 2.3: Relation of uniform sampling density to completeness of the recovered model, top row: feature space and sample points (red), bottom row: recovered surface patches (red) associated with sampled points. 


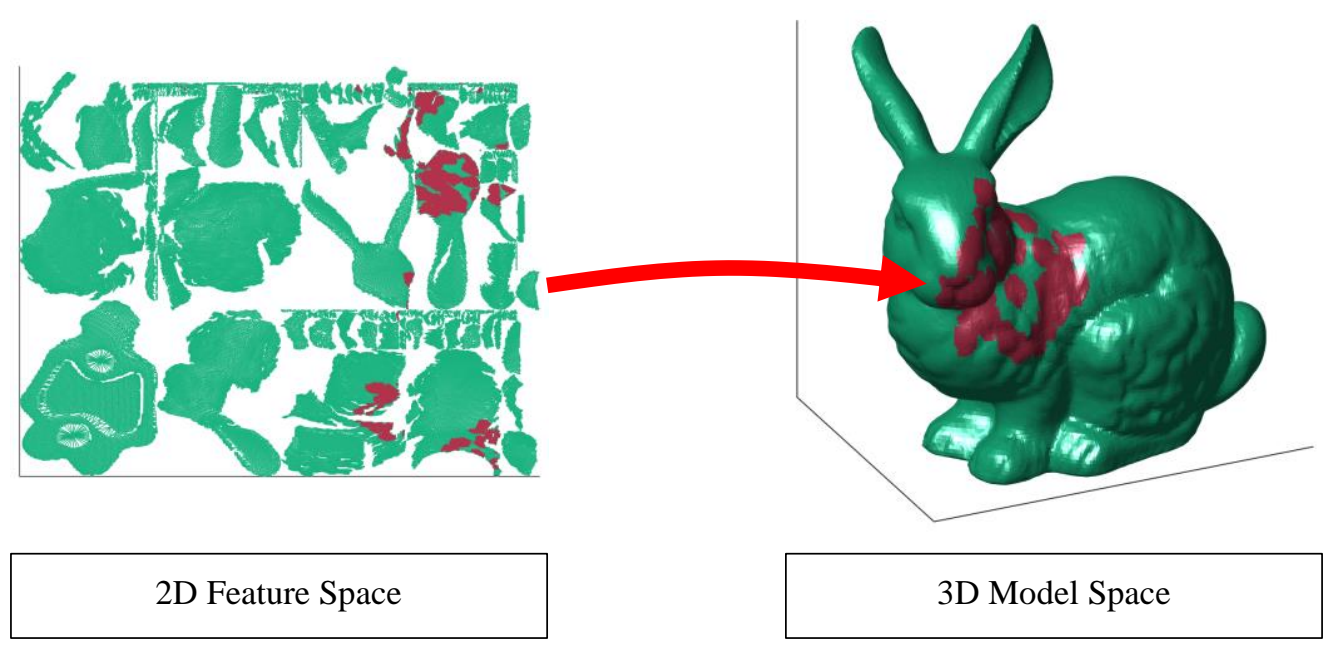

Figure 2.4: Left: non-uniform feature space sampling, right: associated object patches recovered.

The accuracy and completeness of the shape recovered depend on the model generation method, the number of cameras, and camera configuration. Specifically, the model generation method will directly influence the accuracy of the recovered shape, while the combination of model generation and camera positioning methods will influence completeness. For example; many properly placed, well-calibrated, static cameras can maximize both objectives at the cost of processing power. The performance of the cameras will decrease in the presence of obstacles, or if the number of cameras is reduced, or both. In contrast, reconfigurable cameras can potentially improve recovery performance by proactively changing the cameras' position, orientation and optical parameters to overcome expected occlusions. Further, the total number of reconfigurable cameras necessary can be significantly less than the static camera case.

The static camera case for maximizing shape recovery objectives can be addressed through workspace saturation, namely, increasing the total number of cameras to improve visibility without regard to obstacle occlusions or object self-occlusions. Alternatively, the reconfigurable camera case can be framed as an optimization process that tries to maximize a visibility-based objective function:

$$
\begin{gathered}
\text { given } \mathbf{P}_{c}(t) \\
\max _{\mathbf{P}_{c}(t+1)}(\Gamma(t+1)) \\
\operatorname{sub} \mathbf{L}(t),
\end{gathered}
$$

where $\mathbf{P}_{c}(t)$ is the matrix of camera parameters at the current demand instant $t, \Gamma$ is the visibility objective function, and $\mathbf{L}(t)$ is the set of workspace and camera constraints imposed on the system at the current demand instant. The optimization attempts to locate the set of camera parameters for the next demand instant that maximizes the objective function. The major advantage of reconfigurable camera 
systems is their ability to overcome the object's self-occlusions and obstacle occlusions to improve shape recovery. Reconfigurable cameras will remain the focus of this dissertation, while static cameras will be referenced for comparative purposes.

Section 2.1 concluded that a visibility-based objective function is the best generalized approach for the accuracy and completeness objectives of shape recovery. Thus, for reconfigurable cameras, the reconfiguration must be based on optimizing camera parameters to maximize the object's visibility. A caveat of this optimization is that it must be done for the next demand instant, $t+1$, instead of the current, $t$. This is important as optimizing for the current recovered model will not guarantee an improvement in performance at the next demand instant. Specifically, since cameras are limited in acceleration and velocity, they cannot reconfigure instantaneously to the near-optimal configuration that would maximize performance at the current demand instant. Therefore, by optimizing the camera configuration for the expected object's deformation, the camera motion constraints are considered for performance improvement.

A multi-camera active-vision system must accomplish three main tasks to ensure the shape recovery objectives are maximized for a priori unknown deforming objects. These tasks include model generation, deformation estimation, and camera reconfiguration. The objective function development and analysis tasks are grouped with camera reconfiguration since they are a part of the reconfiguration task. The remainder of this chapter will overview the challenges of each individual task.

\subsubsection{Model Generation}

The model generation task must produce the most accurate model, $\mathbf{M}$, of the target object through a passive multi-camera system. A passive system implies that model generation is based solely on the images produced without pattern projection or light-source manipulation. Image segmentation techniques are outside the scope of this work. The segmentation of the target object and obstacles from the background is assumed to be handled by an external, on-line process. The generated model must be compatible with the deformation estimation process and the objective function.

The accuracy of the generated model will explicitly depend on the shape recovery methodology developed. For example, triangulation methods can produce better surface accuracy than visual hull techniques for the same number of cameras. In contrast, a small number of well-placed cameras can produce a complete volumetric estimate of the target object through a visual hull, while triangulation may fail. The model completeness objective can, theoretically, be better handled by the visual hull approach than the triangulation approach at the high cost of accuracy. Further, various approaches to triangulation such as fully calibrated cameras versus partially calibrated cameras induce their own disadvantages such as frame 
of reference ambiguities. Therefore, the model generation task must strive to maximize the accuracy of the recovered shape and attempt to model the complete object at the current demand instant.

Another consideration for model generation is the model's representation. Each type of recovery method produces its own data set, whether a point cloud from triangulation or a volumetric voxel-based object for visual hulls. These representations may be converted into alternatives such as surface polygons or parametric surfaces. An example of point cloud, polygon and voxel models is presented in Figure 2.5. Each individual model representation would require specific deformation estimation and reconfiguration methodologies. Thus, these considerations must be taken into account when developing the model generation method necessary for the camera reconfiguration methodology for a priori unknown deforming objects. Specifically, the model generation method developed should generalize each kind of target object, either surface-based, or volume-based.
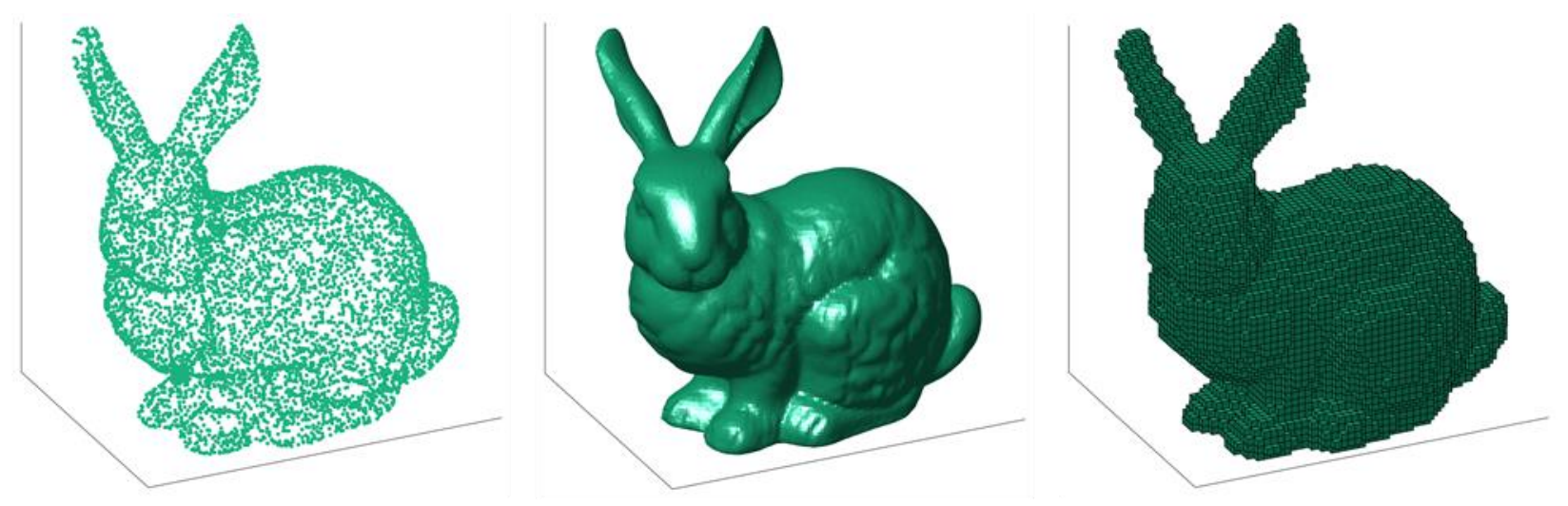

Figure 2.5: Left: point cloud representation, middle: polygon surface representation, right: voxel representation.

\subsubsection{Deformation Estimation}

The deformation estimation method must accurately predict the object's model at the next demand instant, $\mathbf{M}^{+}$. An accurate deformation estimation method relies on a robust tracking and prediction algorithm. Tracking consists of temporal feature matching and motion estimation. Within the context of deformation estimation, the tracking method must create a correspondence between the models generated at the current and previous demand instants. A prediction method must then estimate the deformation of the model for the next demand instant. The shape recovery goals apply to the deformation estimation as well, namely, the predicted deformation must maximize the accuracy and completeness of the expected model. Similarly, the tracking method must maximize the accuracy of correspondence. 
The tracking and prediction methods must both be robust to varying number of tracked features and object size. The methods must scale for the following variables: number of cameras available, number of detected features, and number of tracked features. The tracking precision and prediction precision should improve depending on the scale of the motion observed in each dimension, namely, smaller motion should result in smaller tracking and prediction variance. The prediction method must implement a motion model that can accurately capture the object's motion. Ideally, the motion model should also capture motions that are outside of the model, for example, a polynomial motion model capable of capture a part of a sinusoidal motion. The deformation estimation must implement the same model representation method as the model generation method to maintain compatibility across the complete methodology.

\subsubsection{Reconfiguration}

The reconfiguration method must evaluate a visibility-based objective function over the set of all possible camera parameter combinations, select the near-optimal, and plan the camera motions. The method consists of several individual problems that must be addressed. These include:

i) the discretization of the workspace and camera parameters,

ii) the development of the visibility-based objective function,

iii) the evaluation of the objective function for various camera parameters combinations,

iv) the selection of the near-optimal camera parameter combination,

v) and the reconfiguration path planning.

The workspace discretization process must produce a set of possible camera parameters for each camera, and the superset of all combinations of camera parameters, Figure 2.6. Each parameter sets must uniformly sample each camera's workspace limits to remove spatial bias. Herein, the camera's workspace is defined as the finite range of each modifiable camera parameter such as $x$-pose, $y$-pose, $z$-pose, rotations, focal length, and focus distance. A parameter set is the unique combination of each modifiable camera parameter, represented by the white rectangles in Figure 2.6.

The total number of parameter sets for each camera must be kept reasonable. A reasonable number of sets will be dictated by the total number of individually varying camera parameters, and software processing limitation. Specifically, for $c$ number of cameras, each having $k$ number of parameter sets, the total number of camera parameter combinations becomes: 


$$
C_{\text {comb }}=k^{c} \text {. }
$$

A large number combinations may become computationally prohibitive since each combination must be processed through the objective function. The superset of all combinations of camera parameters includes every possible combination of one parameter block from all available cameras, one such combination represented by the orange outline in Figure 2.6.

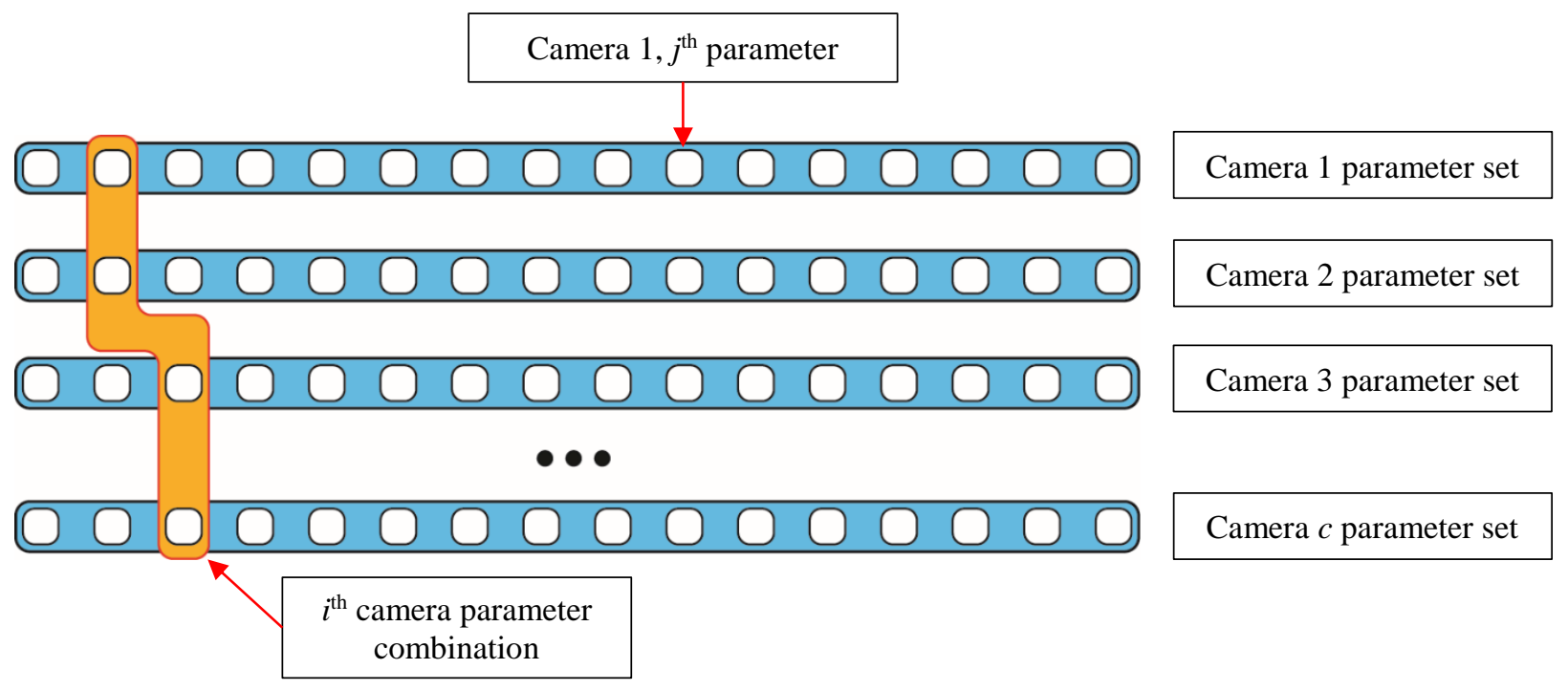

Figure 2.6: Illustration of the camera parameter sets and one camera parameter combination.

The objective function must be developed through a visibility estimation as outlined in Equation (2.5). The visibility-based objective function optimization will focus on model completeness. The function must correctly estimate the surface visibility of the expected model's deformation for a given camera parameter combination. Further, this function must be robust to the number of cameras in the system, the object's self-occlusion, and any obstacle occlusions.

The evaluation of the objective function for each camera parameter combination must produce a single, scalar value, namely, the estimated surface area visible. Since there exist up to $C_{c o m b}$ potential camera parameter combinations, the objective function evaluation method should be developed to reduce the total number of computations required. By reducing the total number of combinations, the size of the individual camera parameter sets could be increased. The near-optimal camera parameter combination will be chosen to maximize the total surface area visible while ensuring that shape recovery on the visible surface area can be performed. Once a near-optimal camera parameter combination is chosen, the cameras' paths must be planned from their current position and orientation to their near-optimal. 
Camera path planning must produce a piece-wise smooth trajectory of the camera's pose and orientation. The trajectory must avoid workspace obstacles, and ensure the camera's orientation maintains the target object within the FOV. Further, the motion steps along the path must be planned to ensure image motion blur is reduced, and tracking remains feasible, namely, if the camera's motion steps are too large, the tracking correspondence may be lost, resulting in the inability to estimate the object's deformation. Once the paths are planned for each camera, all cameras can be moved along their paths for the next demand instant.

\subsection{Summary}

The accuracy and completeness objectives of shape recovery were herein generalized in terms the surface sampling accuracy and surface area visibility, respectively, for a multi-camera active-vision system for $a$ priori unknown objects. The system must complete three major tasks, namely, model generation, deformation estimation, and reconfiguration. The model generation method will focus the passive multicamera active-vision task of producing the 3D model of the target object at the current demand instant. The model generation task must maximize the accuracy objective of shape recovery for the a priori unknown object. The deformation estimation task must use the generated model, gather tracking data, and produce an estimate of the expected object's deformation for the next demand instant. The reconfiguration task will then use the expected deformation to evaluate a surface visibility metric for each camera parameter combination, select the near-optimal combination, and plan the cameras' paths. The tasks must be developed such that input and output data type are compatible. The three tasks must be addressed within a generalized framework such that the overall methodology could be applied in any scenario with minimal modifications.

The remainder of the dissertation will describe, in detail, the approach developed to address the problem of shape recovery of a priori unknown deforming objects in dynamic environments. The methodology will be framed within the three major tasks described in this section, namely, model generation, deformation estimation, and reconfiguration. Each task will then be presented and explained in detail. Simulations and experiments will then be presented to supported the methodology developed. 


\section{Chapter 3 \\ Methodology: Model Generation}

The problem of shape recovery of a priori unknown deforming objects in dynamic environments has been framed as a visibility optimization for a multi-camera active-vision system. The system must include a model generation method, a deformation estimation method, and a reconfiguration method. Reconfigurable systems provide an advantage over static systems wherein obstacle occlusions and object self-occlusions can be overcome through camera placement. Further, reconfiguration can be used to reduce the total number of cameras a static system may employ to saturate the visibility and relax the workspace constraints.

This chapter will present the overview of the generic methodology developed for the shape recovery of a priori unknown deforming objects through a multi-camera active-vision system. This chapter will focus on the model generation method developed. Chapters 4 and 5 will describe in detail the deformation estimation and the reconfiguration methods, respectively.

\subsection{Methodology Overview}

The proposed shape recovery methodology is presented in Figure 3.1. The process begins by acquiring images from $c$ number of cameras as a data structure of images $\mathbf{I}(t)$ at the $t^{\text {th }}$ demand instant. The image data structure and current camera parameter data structure $\mathbf{P}_{\mathrm{c}}(t)$ are parsed to the model generation method. The model generation method extracts the image data and through a fusion process produces a complete estimate of the complete shape of the object, $\mathbf{M}(t)$. The model data is copied into the memory database and parsed to the deformation estimation method alongside the current camera parameters.

The deformation estimation method implements an adaptive particle filter tracking algorithm to estimate the expected position of tracked features in 3D space and then fuses the estimate with the model generated to produce the expected model deformation at the next demand instant, $\mathbf{M}^{+}(t)$. The expected model deformation and current camera parameters are passed to the reconfiguration method and stored in the database.

The reconfiguration method discretizes the camera parameter workspace and evaluates the visibility-based objective function for all possible camera parameter combinations. It then selects the nearoptimal combination that is expected to maximize surface visibility and improve shape recovery accuracy. The cameras' paths are then planned and the cameras are moved.

Each component of the methodology parses a specific data set to its dependent components. The image capture process sends a data structure of all images captured at the $t^{\text {th }}$ demand instant from $c$ cameras 
and the camera parameter matrices consisting of position, orientation, and calibration data. The model generation method produces the current object's model $\mathbf{M}(t)$ and passes through the camera parameter matrices to the deformation estimation. The deformation estimation produces an expected deformation model $\mathbf{M}^{+}(t)$ and pass the camera parameter matrices. The reconfiguration method uses the available camera data and expected model deformation to reposition all the available cameras trying to improve the shape recovery for the next demand instant.

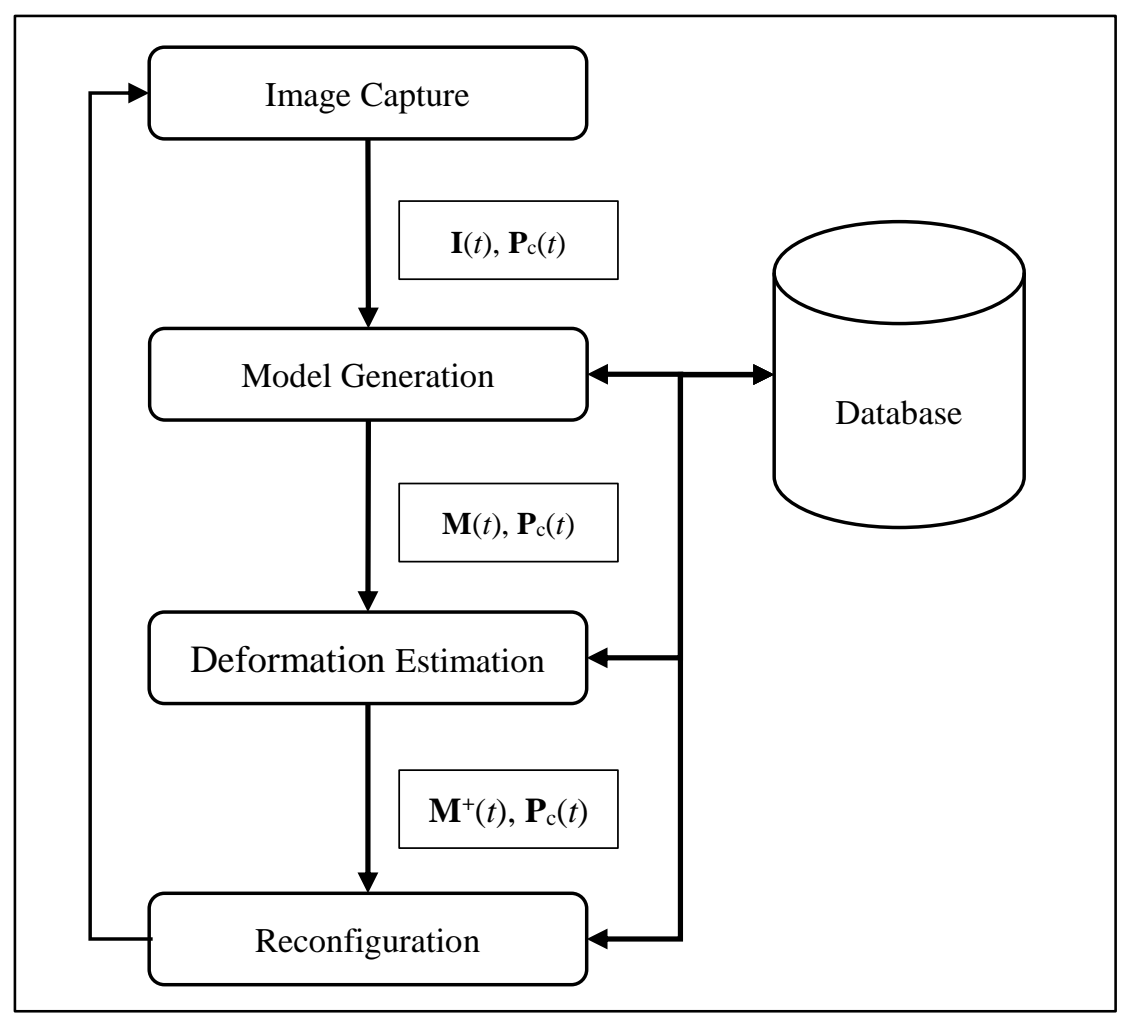

Figure 3.1: Proposed methodology.

The scope of the methodology is to maximize the shape recovery objectives for a priori unknown deforming objects through a multi-camera active-vision system. To that extent, certain out-of-scope tasks and considerations must be explicitly defined. The first out-of-scope task is image-based target selection, specifically, a method for target identification in images will not be developed. This can either be addressed through a user-interface or an automated selection processed, in either case, target selection is implementation-dependent and not considered for a generic methodology. The implementation of target identification will be described in both simulation and experimental cases in the following chapters.

Image-based target segmentation, similar to target selection is also an out-of-scope task. The segmentation process labels image pixels by their classification as either target, obstacle, or background. Segmentation is a well-studied computer-vision problem, with numerous proposed solutions. Due to the 
breadth of available techniques and associated machine learning problems, segmentation is outside of the scope of this work. The methodology developed assumes that the target is separable in the images from the background and obstacles and that segmentation data is available on-line during run-time. The implementation of segmentation techniques will be described in both simulation and experimental cases in the following chapters.

The final consideration of this work is the application of solid-based objects. The problem of shape recovery was defined for both solid-based and surface-based objects. This work will focus on solid-based objects. The application of the system towards surface-based objects will be considered in Chapter 8 .

\subsection{Model Generation Overview}

Model generation methods include triangulation, visual hulls, or fusion techniques. The nature of the problem requires the model generation method to produce the most accurate and complete model of the target object without a priori knowledge of the object's identity. A generic approach to the problem may include cases where cameras are poorly positioned about the target object, Figure 3.2, therefore triangulation methods would recover incomplete surface patches while a visual hull would over-estimate the bounding volume. In contrast, a fusion technique that combines triangulation with a visual hull would produce a model with highly accurate triangulated surface patches and a complete estimate of object's volume.

Model generation for a priori unknown objects implies that an accurate measurement between the recovered shape and the true model is not possible during run-time from the system's perspective. The model's accuracy could be measured for test objects whose model is known to the user, but during the system's run-time, a ground truth model is unavailable. Therefore, the model generation method must inherently attempt to maximize the recovery accuracy and completeness through the implemented shape recovery technique.

A novel method that fuses information from passive range data and a visual hull to estimate the 3D deformation of an unknown object is proposed herein, Figure 3.3. For each demand instant, the following set of tasks is completed: (i) stereo-camera pair selection for triangulation, (ii) triangulation, (iii) selection of camera pairs for visual hull boundary estimation (iv) visual hull boundary estimation, (vi) visual hull building and (vii) model fusion. The method is scalable for a varying number of cameras in the workspace and is independent of the target object's shape. The implementation focuses on homogenous solid objects. 

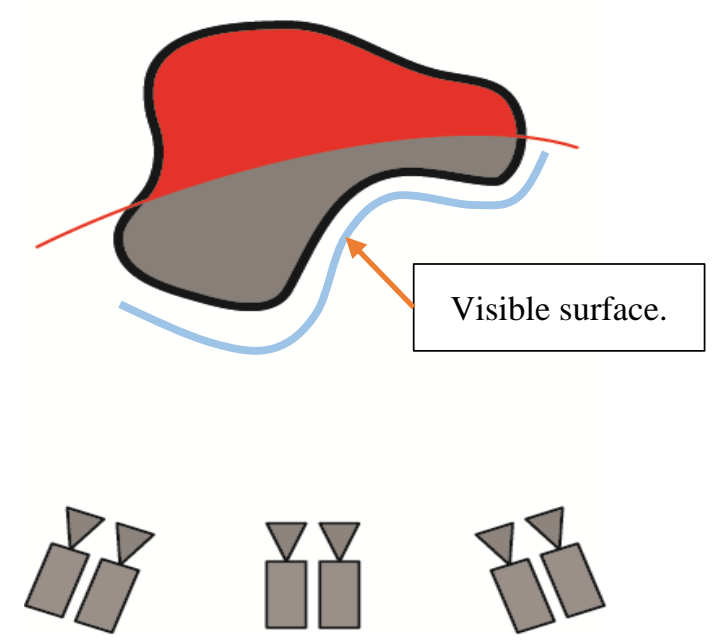

Figure 3.2: Example of poorly positioned cameras.

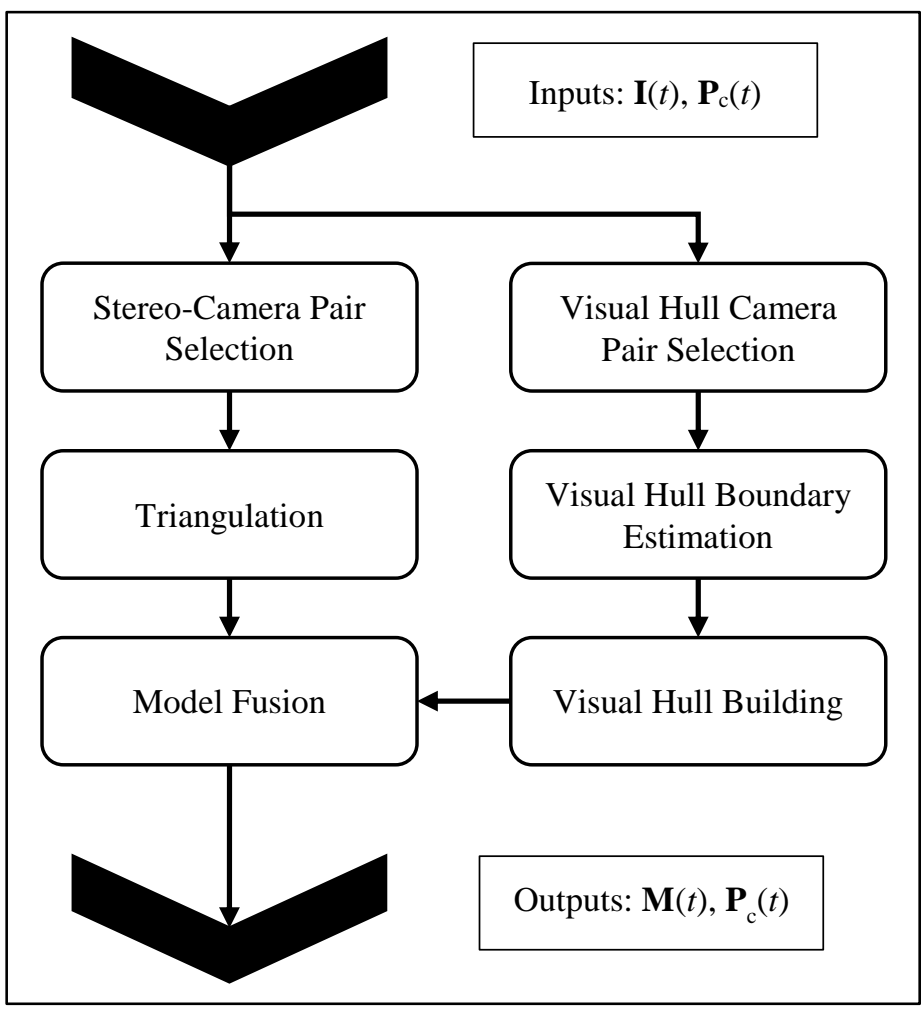

Figure 3.3: Model generation methodology. 


\subsection{Stereo-Camera Pair Selection}

The proposed methodology allows for a variable number of cameras in the workspace without a priori designation of stereo-pairs. The stereo-camera pair selection algorithm determines which cameras will be used for stereo triangulation. It is necessary to choose cameras that are positioned closely and oriented in a common direction. This task selects all viable stereo-camera configurations for $c$ number of cameras based on their baseline separation, $d_{\text {baseline }}$ and $\theta_{\text {baseline }}$ and angular separation, Figure 3.4.

First, all possible camera pairs are stored into a camera-pair-index list, $\mathbf{K}_{\max }\left[2 \times k_{\max }\right]$, with the total number of pairs, $k_{\max }$, determined by:

$$
k_{\max }=\frac{c !}{2 !(c-2) !} .
$$

Then, for each combination three parameters are tested: correct optical axis orientation, baseline distance, $d_{\text {baseline }}(k)$ and angular separation, $\theta_{\text {baseline }}(k)$. The correct orientation is tested by locating the closest point of the optical centers of both cameras in space, wherein $\boldsymbol{u}$ and $\boldsymbol{u}$ ' represent the optical axes of each camera. This point, $\boldsymbol{p}^{*}$, must lie in front of both cameras. The angular separation is calculated as:

$$
\theta_{\text {baseline }}(k)=\cos ^{-1}\left(\frac{\left(\boldsymbol{p}^{*}-\boldsymbol{o}\right) \bullet\left(\boldsymbol{p}^{*}-\boldsymbol{o}^{\prime}\right)}{\left\|\left(\boldsymbol{p}^{*}-\boldsymbol{o}\right)\right\|\left\|\left(\boldsymbol{p}^{*}-\boldsymbol{o}^{\prime}\right)\right\|}\right),
$$

Where $\boldsymbol{o}$ is the position of the left camera, $\boldsymbol{o}$ ' is the position of the right camera and $\theta_{\text {baseline }}(k)$ is the angular separation between the optical centers of the $k^{\text {th }}$ camera pair. A satisfactory stereo camera pair has a baseline that is less than a threshold distance $d_{b-\max }$ and an angular separation of less than a threshold angle $\theta_{b-\max }$.

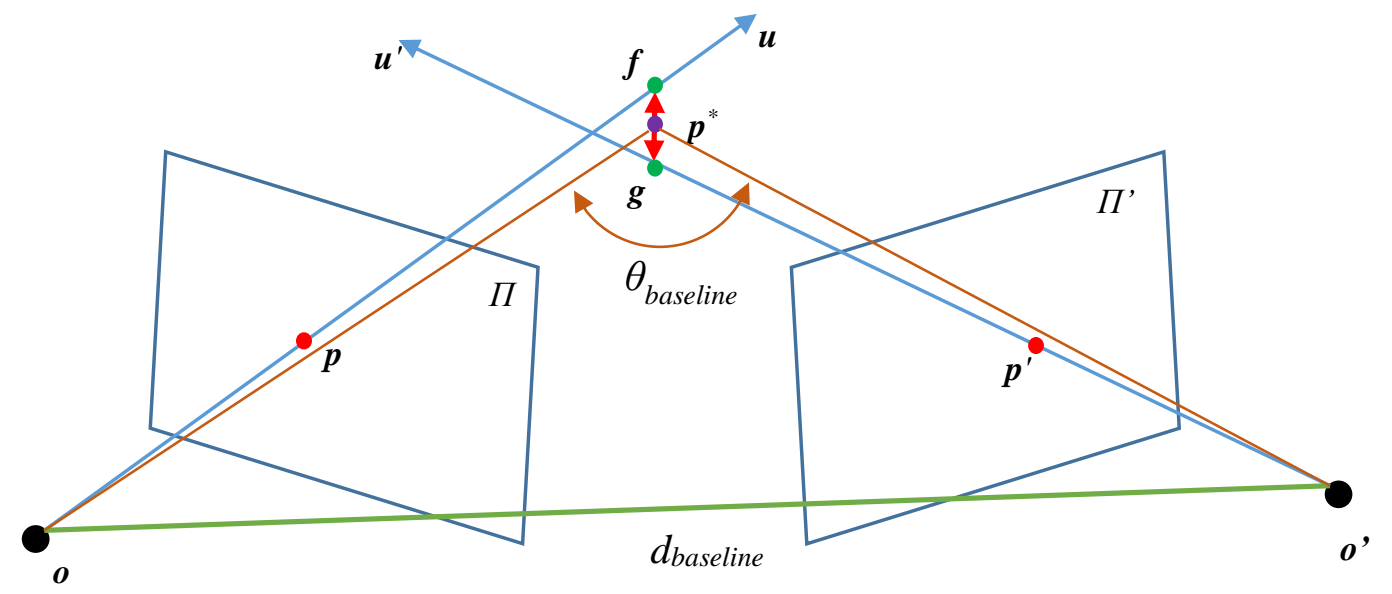

Figure 3.4: Stereo triangulation definition. 
The thresholds $d_{b-\max }$ and $\theta_{b-\max }$ must be set according to the stereo matching method chosen, namely, if dense, photo-metric methods [5], [49], [51] are used, then, the baseline and angular separation must be minimized to preserve view-point photometric consistency and reduce affine distortion [51]. Conversely, robust, scale-invariant features such as scale-invariant feature transform [83], [84] (SIFT) or speeded-up robust features [85] (SURF), or alternatives [56], allow for larger baseline and angular separation due to their feature matching robustness under affine distortions [84]. The resulting list of stereocamera pairs is stored in $\mathbf{K}_{T R}$, a [2 $\left.2 k_{T}\right]$ matrix, where $k_{T}$ is the number of viable pairs.

\subsection{Triangulation}

Triangulation methods rely on matching image features between image sets to create inter-image correspondence. Once 2D features in images are correctly corresponded, 3D rays are projected into the workspace for each set of corresponding $2 \mathrm{D}$ image features. The rays are used to triangulate the 3D location of a matched feature by locating the midpoint, $\boldsymbol{p}^{*}$, of the shortest distance between the rays. Figure 3.4 illustrates the concept of triangulation, where $\boldsymbol{o}$ and $\boldsymbol{o}$ ' are the locations of two cameras, $\boldsymbol{p}^{*}$ is the location of the $3 \mathrm{D}$ feature, $\boldsymbol{p}$, and $\boldsymbol{p}^{\prime}$ are the corresponding features in the image planes, $\Pi$ and $\Pi$ '. Three distinct cases of triangulation exist: triangulation from fully calibrated cameras, triangulation from intrinsically calibrated cameras and triangulation from uncalibrated cameras. Triangulation from partially calibrated cameras is generally referred to as structure from motion (SFM) [1].

\subsubsection{Camera Calibration}

Triangulation from fully calibrated cameras directly solves for the 3D point location $\boldsymbol{p}^{*}$. The rays $\boldsymbol{u}$ and $\boldsymbol{u}^{\text {' }}$ can be directly computed from the a priori known intrinsic and extrinsic camera calibration matrices. The points $\boldsymbol{f}$ and $\boldsymbol{g}$ are defined as:

$$
\begin{gathered}
\boldsymbol{f}=\boldsymbol{o}+\lambda \boldsymbol{u}, \\
\boldsymbol{g}=\boldsymbol{o}^{\prime}+\lambda \boldsymbol{u}^{\prime},
\end{gathered}
$$

where $\lambda$ and $\mu$ are scaling factors. Orthogonality constraints are considered such that:

$$
\begin{aligned}
& (\boldsymbol{f}-\boldsymbol{g}) \boldsymbol{u}^{T}=0, \\
& (\boldsymbol{f}-\boldsymbol{g}) \boldsymbol{u}^{\prime T}=0,
\end{aligned}
$$

where $\left(^{T}\right)$ is the transpose operator, the points $\boldsymbol{f}, \boldsymbol{g}$ and the unit vectors $\boldsymbol{u}, \boldsymbol{u}$ ' are $[3 \times 1]$. Equations (3.3) (3.6) can then be converted into a linear system and solved for with respect to $\lambda$ and $\mu$. Then, using $\lambda$ and $\mu$, the exact locations of points $\boldsymbol{f}, \boldsymbol{g}$, and $\boldsymbol{p}^{*}$ can be solved. This formulation is one approach to stereo 
triangulation. The least squares minimization approach should be used for multi-camera triangulation where a single $2 \mathrm{D}$ feature corresponded between more than 2 cameras.

The major benefit of calibrated camera triangulation is the accuracy of locating 3D points in space. Similarly, calibrated camera systems can strictly enforce epipolar geometry assumptions. The epipolar geometry assumption states that the ray $\boldsymbol{u}$ can be directly projected onto $\Pi$ ' as a line, thus, the corresponding 2D point $\boldsymbol{p}$ ' should be located along the projection of $\boldsymbol{u}$ onto $\Pi$ '. The calibration data also provides accurate positioning of the camera and sensor locations in space, improving the accuracy of ray projection. Triangulation errors in calibrated systems arise from feature correspondence errors and residual calibration errors. The disadvantage of fully calibrated camera triangulation is the necessity of the calibration process. Further, calibration errors can increase triangulation error when cameras are not calibrated as stereo pairs.

The partially calibrated and non-calibrated triangulation approach, or SFM, requires algorithmbased solutions such as the eight-point algorithm [163], bundle-adjustment [164], or singular-valuedecomposition (SVD). These methods rely on a minimum set of 2D image feature correspondences to estimate either the Essential or Fundamental matrices representing the rigid transformations between the cameras. The advantage of SFM methods is that full camera calibration is not necessary. SFM can be applied to intrinsically calibrated cameras without extrinsic parameters to estimate the Essential matrix and then triangulate $2 \mathrm{D}$ matched features into $3 \mathrm{D}$ coordinates.

SFM for uncalibrated weak-perspective cameras estimates the Fundamental matrix which is used to solve the relative $3 \mathrm{D}$ coordinates of $2 \mathrm{D}$ points. A benefit of SFM for uncalibrated cameras is that it can be applied as a simple and effective method for handling multiple cameras in a uniform way. The disadvantage of SFM methods is their shape ambiguities including coordinate frame, scale, affinity and projectivity [165].

Coordinate frame ambiguity occurs when triangulated data is relative to the cameras from it was recovered, not relative to a global frame. A lack of a coordinate frame when recovering shape through SFM can be degrading to a multi-camera system when sets of cameras are used to recover independent surface patches. With frame ambiguity, it becomes increasingly difficult to associate the shape recovered from camera set 1 to camera set 2 unless feature matching can be done between the camera sets. This may not be feasible for camera sets that are separated by a large distance about the target object, as is necessary for a reconfiguration method with a limited number of cameras.

Scale ambiguity only preserves the angles and relative lengths between triangulated points. The scale ambiguity for perspective cameras suggests the true scale of the recovered shape may not be recovered, thus the relative geometry of the recovered shape would be consistent with the true object, but 
the scale would be indeterminate without added scene information. The same problem applies with coordinate frame ambiguity, except the relative scales of the recovered shapes from each camera set may differ.

Affine ambiguity would preserve the parallelism and volumetric ratios observed and projective ambiguity would preserve tangency and intersections. Thus, even though triangulation from partially calibrated cameras avoids the typically lengthy calibration processes, it does present its own deficiencies in recovery. As with calibrated cameras, the errors in triangulation stem from the accuracy of the Essential and Fundamental matrix solutions and the correspondence errors.

Within the context of this dissertation, the use of uncalibrated or partially calibrated cameras is not feasible. The implementation of such cameras would result in non-resolvable shape recovery ambiguities between stereo-camera pairs. Specifically, one stereo-camera pair's recovered shape may not be accurately located in world coordinates in relation to another pair's recovered shape. The complete set of surface patches recovered from the camera pairs could not be correctly merged unless the surface visibility explicitly overlapped between neighboring camera pairs. The overlapping condition could potentially limit the completeness metric during reconfiguration for a smaller number of cameras available to the system. Further, the triangulation accuracy of partially calibrated and uncalibrated cameras will depend on the accuracy of the matched 2D features, thus, any matching error will directly carry over into triangulation errors.

In contrast, fully calibrated cameras can operate in independent stereo pairs and produce surface patches within a singular world coordinate reference frame without overlapping surface visibility. The triangulation errors from fully calibrated cameras are independent, namely, the matching errors are separate from calibration errors. Fully calibrated cameras were chosen for the methodology due to their independent pair implementation. The objective of shape recovery accuracy is dependent on the triangulation implementation. Thus, the selection of fully calibrated cameras instead of partially calibrated and uncalibrated cameras ensures that the maximum possible accuracy is obtained during triangulation for $a$ priori unknown objects.

\subsubsection{D Feature Selection}

Triangulation methods rely on the pixel-based correspondence between a set of two or more images. These corresponding image features are then triangulated to produce $3 \mathrm{D}$ coordinates that estimate surface points on the target object, i.e.: features $\boldsymbol{p}$ and $\boldsymbol{p}$ ' in Figure 3.4. The accuracy of correspondence directly correlates to the $3 \mathrm{D}$ triangulation accuracy. Therefore, it is desirable to select features that can be accurately corresponded to improve triangulation. 
Image features compress an image patch into a lower-dimension. The patches can be represented through orientation-locked features such as normalized cross-correlation (NCC) and kernel filters, or orientation-free features such as principle component analysis (PCA), SIFT, SURF, GHOL and their derivatives. Orientation-locked features require the same orientation of the patch in all images for correct correspondence. Therefore, the cameras must be horizontally or vertically aligned, or image pairs must be rectified to ensure epipolar lines are aligned.

Corresponding orientation-locked features typically consists of choosing a patch template in one image and searching for the most similar patch in the second image. The search produces a 2D likelihood measurement over the second image and the coordinates with the highest likelihood are chosen as the match. These features tend to produce dense image matches between closely position cameras [5]. Each feature in one camera pair requires a complete search of the second image which adds processing overhead, specifically, for $n$ features located in the first image, the complete second image must be searched $n$-times. These features typically experience performance degradation under varying lighting conditions and increasing camera baselines (the distance between two cameras in space). Further, proper correspondence requires image rectification which adds processing over-head to the correspondence task. Rectification for fully calibrated cameras can be established deterministically, while partially or un-calibrated cameras require a basic set of matched features.

Orientation-free feature detection methods automatically calculate the scale and rotation of the selected patch feature [83]. These types of features typically store the compressed patch data as a unique integer key. The use of a unique key results in a correspondence process wherein features are detected in each image independently and then matched based on the existence of the same feature in both images. In contrast, orientation-locked features must rescan the second image of the pair multiple times for each feature detected in the first image or the pair. Orientation-free features do not require image rectification and can match features at different scales and rotations. They are, also, typically more robust to the multi-camera baseline length [84]. Most notable examples of such methods have been thoroughly tested for robustness in recall rates [56].

SIFT features were chosen over affine methods [56] and over dense methods [5], [49], [51] since (1) they are the most stable sparse features for tracking implementations [56], [166]-[168] and (2) unlike dense photometric methods, SIFT features do not require a narrow baseline and produce sets of uniquely identified 2D features that may be corresponded over large affine transformations and varying lighting conditions. A comparison of NCC features and SIFT features is presented in Appendix A. The affine-SIFT (ASIFT) feature method [87] could also be used, instead of SIFT, in order to increase the number of stereo 
matches, though, its implementation is currently limited in image size. Similarly, other methods have been shown to operate faster than SIFT at the cost of matching stability [56].

The 2D feature detection process locates all SIFT features for each camera's image at the current demand instant. The features are stored in the database to offload RAM requirements. Each feature is stored with its designated feature descriptor, pixel location, orientation, and the camera with which it was detected. The use of such features constrains the system to objects with a textured surface. For example, flat, single colored objected with no texture would not produce usable features.

\subsubsection{D Matching and Filtering}

The 2D matching consists of locating pairs of 2D features whose keys are most similar though a dot-product operation [84] for each $k^{\text {th }}$ stereo-camera pair in $\mathbf{K}_{T R}$ and filtering removes incorrect matches prior to triangulation. At first, all the previously detected features for the given cameras in the $k^{\text {th }}$ pair are loaded from the database. The chosen feature detector dictates the matching process. The main difference for various feature-descriptors is the size of the key-identifier for the detected features; for example, SIFT features use a 128 8-bit integer key for each feature, while SURF uses a 64 8-bit key. Once the features for the $k^{\text {th }}$ camera pair have been matched, the incorrect matches must be removed through a filtering operation.

The filtering process allows for modularity in the 2D match filter implementation, namely, the filter may be modified, replaced, or combined with another filter to remove outliers produced by the 2D matching process. The overall filter developed herein comprises two serial filters: the epipolar filter and the nearest neighbor filter.

An example of epipolar geometry is presented in Figure 3.5. Epipolar geometry is a special relation between the intrinsic and extrinsic parameters of cameras viewing the same scene. This geometry leads to specific constrains that occur between matched image features. Specifically, two matched features, $\boldsymbol{p}_{s}$ and $\boldsymbol{p}^{\prime}$ lie on the epipolar plane produced by $\boldsymbol{o}, \boldsymbol{o}$, and $\boldsymbol{p}^{*}$. This property can be exploited for match filtering, namely, the rays projected from one camera's view corresponding to $2 \mathrm{D}$ features can be projected on to another camera's imaging plane as $2 \mathrm{D}$ lines. These lines can be used as search vectors along which the corresponding matched $2 \mathrm{D}$ point must exist.

The epipolar filter consists of sorting the matched pairs and checking for the following four conditions: (i) unique left to right match, (ii) multiple left features to single right feature matches, (iii) single left feature to multiple right feature matches and (iv) repeated matches of the same left to right matches. These conditions arise when the feature detector locates more than one feature in the same pixel coordinates (usually at different orientations) or was unable to produce a single best match (Cases ii and iii). All 
redundant matches in Case iv are removed, as they are the same coordinate matches. Then, for each remaining matched pixel-coordinate pair, the epipolar disparity is calculated in pixels by measuring the perpendicular distance from the feature coordinate in the left image to the projected epipolar line of the corresponding feature from the right image, Figure 3.6. Herein, the maximum epipolar disparity, $d_{p-\max }$, was set to $5 \%$ of the square root of the image area in pixels - this ensures the metric is scalable with larger or smaller images. All matches that do not fit the 5\% criterion are removed.

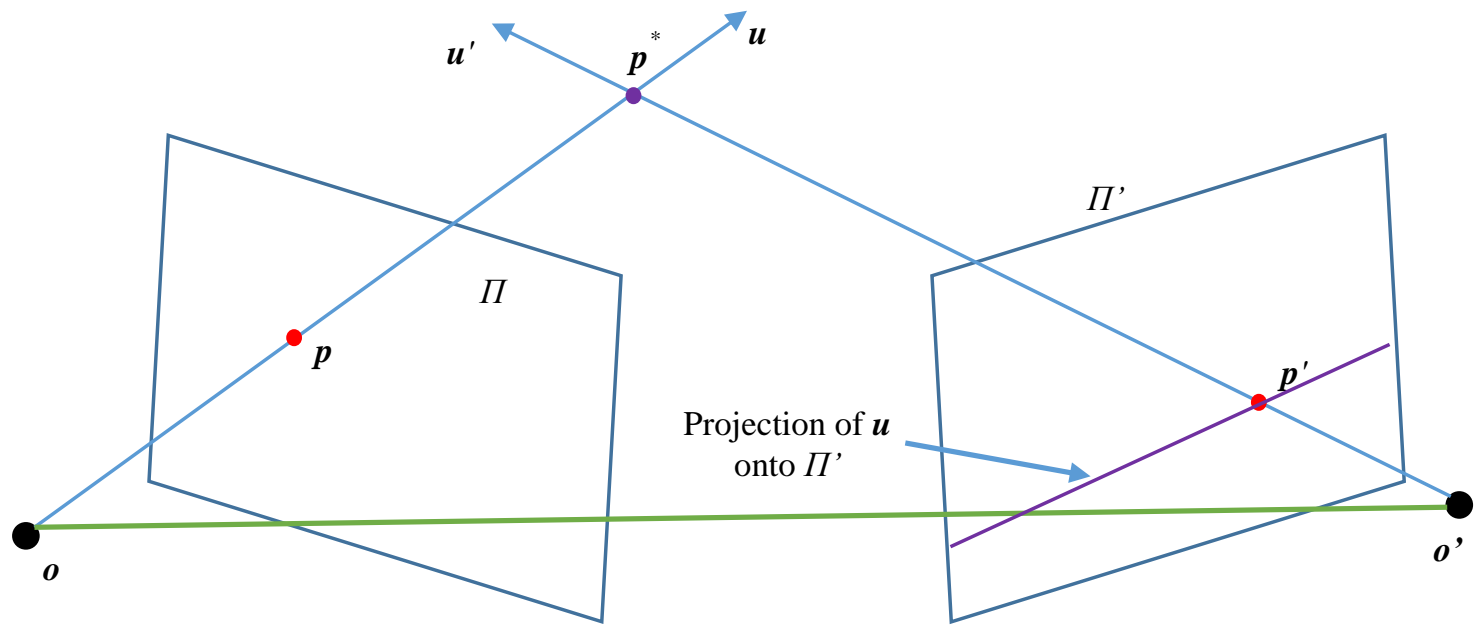

Figure 3.5: Example of epipolar geometry.

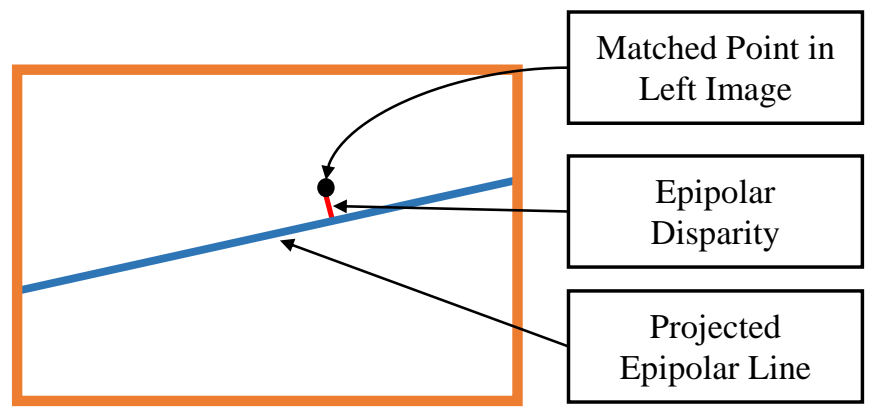

Figure 3.6: Epipolar disparity.

The nearest neighbor filter checks for the consistency of matched 2D features based on the location of the nearest neighbor matches, Figure 3.7. The filter loops over each remaining matched feature pairs in the left image, locates the $n$-nearest of neighbors of a feature and creates a matrix of the vectors from the $j^{\text {th }}$ $2 \mathrm{D}$ feature to the neighbors in the left and corresponding right images, $\mathbf{V}_{\mathrm{L}}[2 \times n]$ and $\mathbf{V}_{\mathrm{R}}[2 \times n]$, respectively. The lengths of all vectors are calculated and stored into vectors $\boldsymbol{d}_{L}, \boldsymbol{d}_{R},[n \times 1]$, which are, then, 
normalized by the sum of all vector lengths and are stored in vectors $\boldsymbol{d}_{L}^{*}$ and $\boldsymbol{d}_{R}^{*}$. The vector angles are calculated as:

$$
\boldsymbol{\varphi}_{L}=\cos ^{-1}\left(\left[\begin{array}{ll}
1 & 0
\end{array}\right] \mathbf{V}_{L}\left[\frac{1}{\boldsymbol{d}_{L}}\right]\right),
$$

which yields an angle from the $x$-axis to each vector connecting the $j^{\text {th }}$ feature to its $n$-nearest neighbors. The angle vectors are, then, normalized by the sum of all angles and stored into vectors $\boldsymbol{\varphi}_{L}^{*}$ and $\boldsymbol{\varphi}_{R}^{*}$. Thereafter, the means and standard deviations of the normalized length and angle vectors are calculated, $\mu_{\varphi_{L}}, \mu_{\varphi_{R}}, \sigma_{\varphi_{L}}, \sigma_{\varphi_{R}}, \mu_{d_{L}}, \mu_{d_{R}}, \sigma_{d_{L}}, \sigma_{d_{R}}$. The final step of the filter is the calculation of errors for each left and right mean and standard deviation:

$$
\begin{aligned}
& e_{\mu_{d}}=\left|\frac{\mu_{d_{L}}-\mu_{d_{R}}}{\mu_{d_{L}}}\right|, \\
& e_{\sigma_{d}}=\left|\frac{\sigma_{d_{L}}-\sigma_{d_{R}}}{\sigma_{d_{L}}}\right|, \\
& e_{\mu_{\varphi}}=\left|\frac{\mu_{\varphi_{L}}-\mu_{\varphi_{R}}}{\mu_{\varphi_{L}}}\right|, \\
& e_{\sigma_{\varphi}}=\left|\frac{\mu_{\sigma_{L}}-\mu_{\sigma_{R}}}{\mu_{\sigma_{L}}}\right|,
\end{aligned}
$$

Above, any feature with a single error over $10 \%$ is removed, as it is most likely an incorrect match. The filters and their respective rejection criteria ensure the removal of most incorrect 2D matches that do not lie along the epipolar lines and most incorrect matches that lie on the epipolar lines but are in the incorrect image region.

The implemented serial filters are a robust approach to filtering and removing incorrect $2 \mathrm{D}$ matches from key-based features. The modularity of the methodology allows for alternative filter implementations and configurations. For example, a random sampling consensus (RANSAC) filter may be implemented in place of the epipolar or nearest neighbor filter, or added as a parallel filter, as it is commonly applied for stereo matching operations in image stitching and model fitting [169], [170]. Alternatively, the optimized random sampling algorithm (ORSA) may be applied in place of both filters to estimate the fundamental matrix and remove matches through the estimated epipolar geometry, [37], [87], [171]. The main difference between the proposed method and methods such as RANCAS or the ORSA is that the developed filter processes stereo matching using the known calibration data. 
Image Left
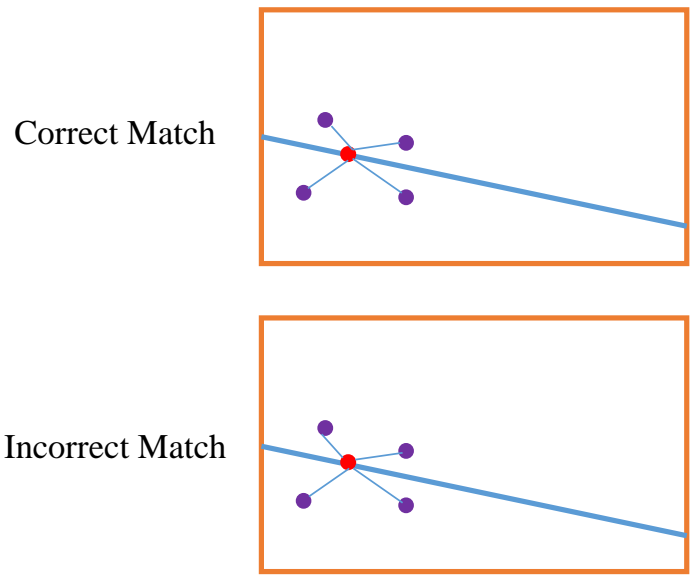

Image Right
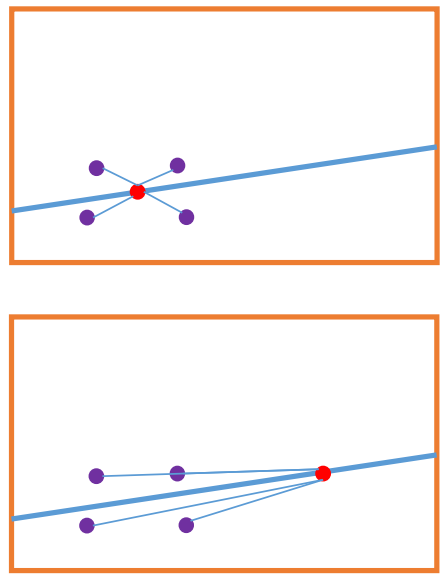

Figure 3.7: Nearest neighbor filter example for stereo-camera pair.

\subsubsection{Triangulation \& 3D Filtering}

The 2D matched, filtered, features for each $k^{\text {th }}$ camera-pair in $\mathbf{K}_{T R}$ are triangulated through ray projection and intersection, Figure 3.4 and Equations (3.3) - (3.6). This yields $k_{T}$-sets of 3D coordinates corresponding to each stereo-camera pair. Triangulation may result in errors of the 3D coordinates, namely, if the 2D filter was not able to remove all outliers, some may remain and appear following triangulation. Therefore, a modular 3D filtering process is proposed herein.

The 3D filter developed for the task is an effective sensing range (ESR) filter, Figure 3.8. The ESR filter removes all triangulated points outside of a stereo sensing range. The filter can operate in two modes - manual or automated. The former allows users to input the 3D range limits for each camera. Thus, any triangulated features outside this range for the $k^{\text {th }}$ camera pair would be removed. The latter mode calculates the depth of field of each camera of the $k^{\text {th }}$ pair and takes the maximum and minimum limits from both cameras as the range plus a user-defined padding percentage, $\kappa$, which adds $\kappa$ times the range of the ESR on both the near and far limits. The ESR filter removes triangulated background artifacts and outliers too close to the cameras.

Triangulation and 3D filtering produces $k_{T}$-sets of point clouds, $\mathbf{X}_{\mathrm{TR}}(k)$, for each $k^{\text {th }}$ stereo-camera pair. Each point cloud is then used to create a triangular surface patch, $\mathbf{T}_{T R}(k)$, of the target object through a Delaunay triangulation. $\mathbf{T}_{T R}(k)$ is herein defined as the triangulation map, wherein triangulation is the graphical subdivision of a planar object into triangles. The map is a size $\left[n_{\text {polys }} \times 3\right]$ index matrix whose rows index the three points from $\mathbf{X}_{\mathrm{TR}}(k)$ that make up a given surface patch polygon. The surface patch is obtained by firstly projecting the point cloud into each camera's image plane as a set of $2 \mathrm{D}$ pixel 
coordinates. One set of 2D coordinates is then triangulated using Delaunay triangulation. The triangulation map is then applied to the second set of 2D coordinates and checked for inconsistencies, namely, incorrect edges. Incorrect edges are a symptom of incorrectly triangulated 2D features. The incorrect edges are removed by removing the $3 \mathrm{D}$ points in $\mathbf{X}_{\mathrm{TR}}(k)$ that connect to the most incorrect edges. The result is a set of fully filtered point cloud matrices $\mathbf{X}_{\mathrm{TR}}(k)$ and their associated surface patches, $\mathbf{T}_{T R}(k)$, Figure 3.9.

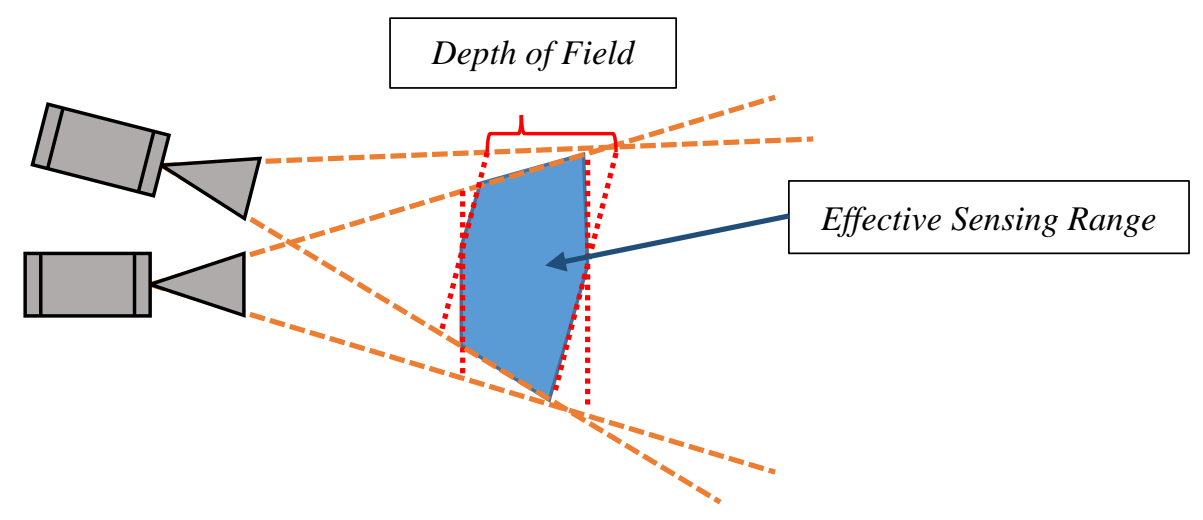

Figure 3.8: Effective sensing range filter for a stereo-camera pair.

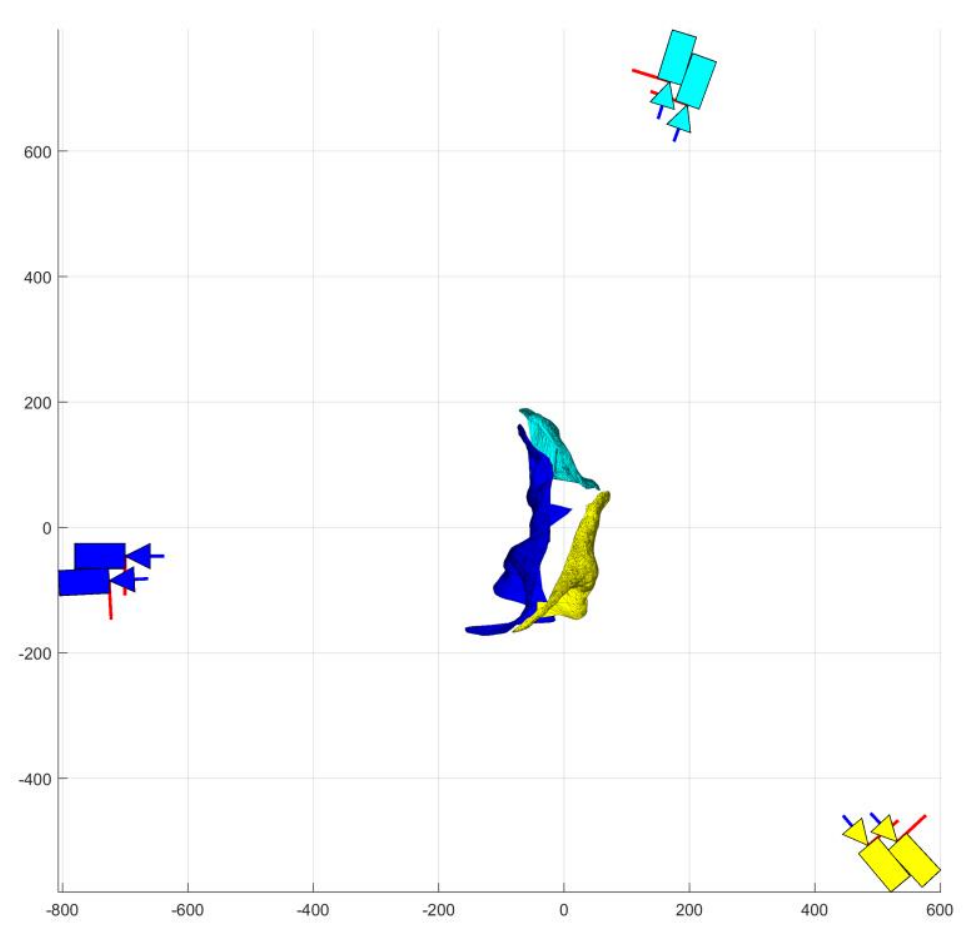

Figure 3.9: Surface patch representation of a deformable object with 3 stereo-camera pairs producing 3 surface patches. 


\subsection{Visual Hull Camera-Pair Selection}

The visual hull building process carves out voxels from an initial boundary volume to produce the volumetric object representation. A priori known objects in the workspace can define the initial boundary volume with little padding necessary. In contrast, a shape recovery system for a priori unknown objects cannot initialize an arbitrary boundary volume in space, as this would either produce an overly large volume or a volume that does not contain the object. Thus, and automatic visual hull boundary generation algorithm was developed. The first step of the boundary generation algorithm is visual hull camera-pair selection. The visual hull camera-pair selection process chooses pairs of cameras in the workspace that would produce the minimal bounding rectangle about the target object. The principle of the visual hull camera pair selection is that perpendicularly orientated cameras would minimize the expected volumetric bounds in two dimensions - similar to orthographic projection in engineering drawings, Figure 3.10. Therefore, three perpendicularly positioned cameras would produce the minimum bounding rectangle for voxel initialization.

The selection algorithm checks the angular separation, $\theta_{\text {baseline }}(k)$ for each $k^{\text {th }}$ camera-pair in $\mathbf{K}_{\max }$ and selects the pairs with angles in the range $\left[-\theta_{v-\max }<90^{\circ}<+\theta_{v-\max }\right]$. The visual hull camera pairs are stored in $\mathbf{K}_{V H}$, a $\left[2 \times k_{V}\right]$ matrix of indices. The inherent assumption is that there exists at least one pair of cameras in $\mathbf{K}_{\max }$ that have an angular separation within the tolerance range. Increasing the tolerance range on the visual hull camera pairs will result in a larger initial boundary volume.

\subsection{Visual Hull Boundary Estimation}

The visual hull boundary estimation determines the size and location of the initial rectangular bounds on the target object prior to voxel initialization. This results in an automated visual hull initialization process with a reduced workspace. To determine the bounds, each image in the visual hull pairs $\mathbf{K}_{V H}$ is fit with a rectangle about the object's silhouette, producing four corner points. Then, for each pairs-of-images, eight $3 \mathrm{D}$ points are triangulated by corresponding specific corner points given the relative orientation of the pairs of cameras, $\mathbf{B}_{V H}$. These eight points produce an initial bounding rectangular prism about the target object. Therefore, for $k_{V}$ visual hull pairs, a set of [ $8 \times k_{V}$ ] 3D boundary points are calculated. The rectangular boundary is chosen as the maximum range in each dimension of the 3D boundary point set, $\mathbf{B}_{V H}$. Figure 3.10 illustrates the initial boundary estimation technique in a birds-eye view; the left case illustrates a smaller boundary produced by orthogonally positioned cameras, while the right case illustrates a larger boundary from non-orthogonally positioned cameras. 


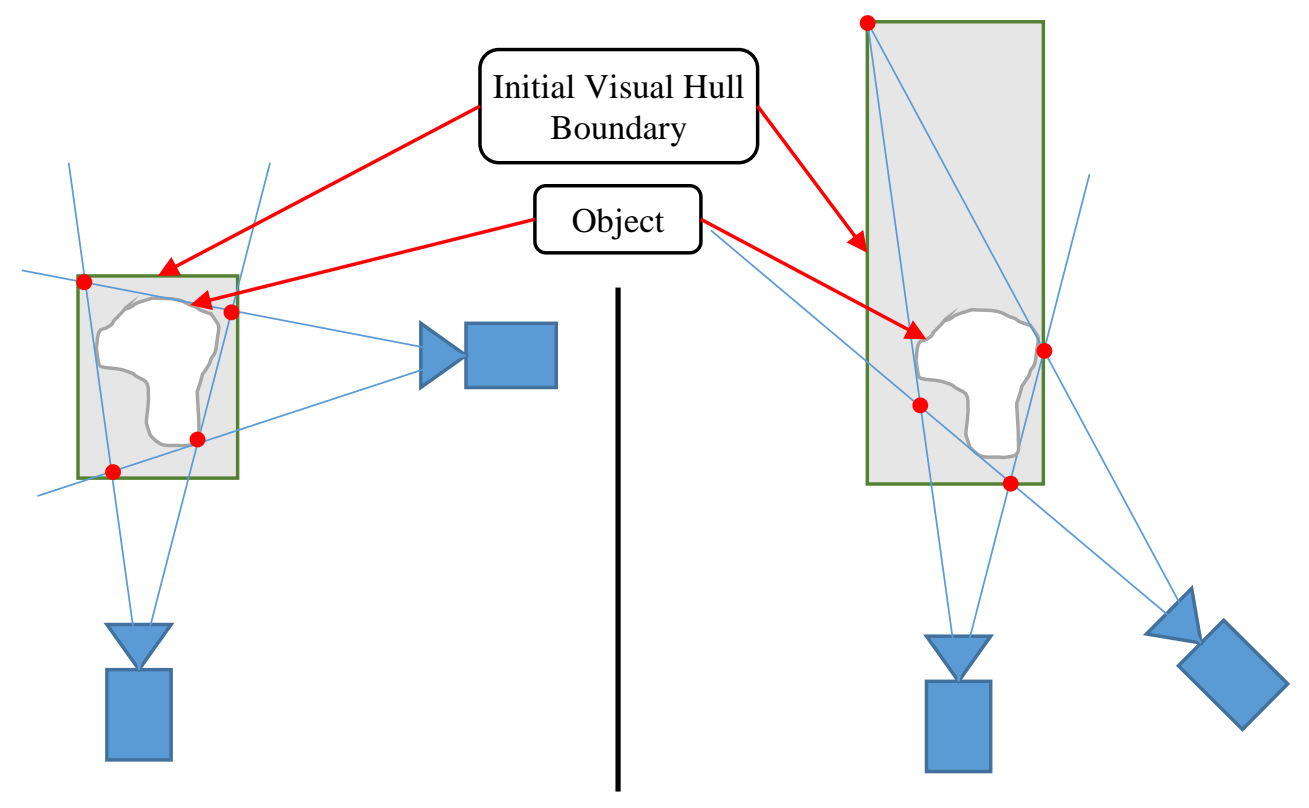

Figure 3.10: Visual hull camera pair selection and boundary estimation.

\subsection{Visual Hull Building}

The visual hull building algorithm uses an iterative process, with a fixed grid and voxel size for each iteration. At first, the maximum grid size in each dimension is specified by $v_{\max }$, along with the rectangular bounds and a maximum number of iterations, $\lambda_{\max }$. Then, for each iteration, the grid size is calculated as:

$$
v(i)=\frac{i \times v_{\max }}{\lambda_{\max }},
$$

where $v(i)$ is the total number of voxels and $i$ is the current iteration. Thus, at each $i^{\text {th }}$ iteration, there are $v(i)^{1 / 3}$ voxels in each dimension. The fineness of the voxels at the final iteration is determined based on the shortest Euclidean distance within the triangulated surface patches to ensure resolution compatibility. For each camera, the voxels are projected into image coordinates and checked whether they are within the silhouette or outside. At the end of each iteration, the voxels within the silhouette are used to calculate the new rectangular bounds for the following iteration. The output of the building process is the set of voxels that are expected to lay inside the target object, $\mathbf{X}_{\text {voxel }}$.

Generally, a visual hull can only produce concave geometry if the concavity is projected onto the silhouette. Thus, the concavities captured through this process are strictly dependent on the placement of the cameras and whether the concavity could be projected onto an image, i.e.: a ' $c$ '-shaped deformation could be captured by a visual hull process, if cameras were correctly positioned, conversely, a semispherical concavity on a face of a cube would not be captured. 


\subsection{Model Fusion}

Triangulation from passive range data locates sets of surface coordinates that pertain to the object, resulting in total confidence of the object's existence in the locale of the triangulation. Conversely, a visual hull produces a bounded estimate of the total volume of the object, within which it exists. The downside of triangulation from sparse features is that the surface area recovered, typically, is not complete. Namely, due to camera placement, some viewpoints of the object may be missed, resulting in an incomplete surface. Therefore, to estimate a complete object shape from triangulation and a visual hull, a fusion method is necessary. The fusion would provide an explicit estimate of the object's surface coordinates from triangulation data and a likelihood estimate of surface existence from visual hull data.

The model fusion process combines the triangulated points, $\mathbf{X}_{T R}$, with the visual hull voxels $\mathbf{X}_{\text {voxel }}$ into a singular model with a surface-based mesh representation. Fusion combines the triangulated surface patches that represent concavities with the visual hull that represents the maximum bounding volume of the object. The process, first, removes all redundant visual hull voxels through a trimming operation. Then, a Marching Cubes [172] algorithm is used to create a mesh over the remaining voxels, producing a set of vertices, $\mathbf{X}_{V H}$ and a visual hull mesh, $\mathbf{T}_{V H}$. The triangulated surface patches, $\mathbf{T}_{T R}(k)$, are then combined with $\mathbf{T}_{V H}$ to create a singular data structure representation of the target object, $\mathbf{T}^{*}$. A set of weights are calculated and attached to all visual hull points within $\mathbf{T}^{*}$ based on their proximity to triangulated points. These weights are necessary for deformation estimation.

The first step of fusion is to remove excess visual hull data in the regions where triangulation data is available. The removal process is carried out $k_{T}$-times, once for each available surface patch $\mathbf{T}_{T R}(k)$. The process is a secondary inverse-carving operation applied to the visual voxels, wherein all voxels located within a polyhedron constructed between the center of the stereo-camera pairs and their associated surface patch are removed. The center of the stereo-camera pairs for the $k^{\text {th }}$ pair is calculated:

$$
\boldsymbol{o}_{c}=\frac{1}{2}\left(\boldsymbol{o}+\boldsymbol{o}^{\prime}\right)
$$

where the naming convention follows Figure 3.4, namely, $\boldsymbol{o}$ and $\boldsymbol{o}$ ' are the locations of the left and right cameras of the given pair, then $\boldsymbol{o}_{\mathrm{c}}$ is the center point between the camera pairs. The remainder of the

polyhedron is constructed by locating the boundary edge of the $k^{\text {th }}$ surface patch, $\mathbf{T}_{T R}(k)$ and connecting each vertex on along the edge to the center point $\boldsymbol{o}_{\mathrm{c}}$. Each voxel in $\mathbf{X}_{\text {voxel }}$ is then tested for its inclusion in the polyhedron using the algorithm developed in [173]. Voxels located within the polyhedron are removed. Figure 3.11 illustrates the voxel trimming process, note that the top most image only shows the user's perspective. The true object's model is unknown to the system throughout its operation. 
The remaining visual hull voxels are processed through the marching cubes algorithm to create a surface mesh representation of the visual hull, $\mathbf{T}_{V H}$ with vertices $\mathbf{X}_{V H}$. The surface patches $\mathbf{T}_{T R}(k)$ are then combined with $\mathbf{T}_{V H}$ to produce a singular data structure $\mathbf{T}^{*}$. Following the addition of the visual hull points, a set of relative weights must be calculated that relate all visual hull points to the triangulated, tracked points. These weights will aid in the deformation estimation process. The weights are represented by an $[n$ $\times m]$ matrix, $\mathbf{W}_{\mathrm{M}}$, that stores the relative influence of each tracked, triangulated point on each visual hull point, for $n$ tracked, triangulated points and $m$ visual hull points. The weights represent the normalized relative distance between each tracked point to each visual hull point:

$$
\mathbf{W}_{M}(i, j)=\frac{d_{e}(i, j)}{\sum_{i=1}^{n} d_{e}(i, j)},
$$

where $i$ is the index of the tracked point and $j$ is the index of the visual hull point and $d_{e}(i, j)$ is the Euclidean distance between the $i^{\text {th }}$ tracked, triangulated point and the $j^{\text {th }}$ visual hull point in the model. The fused model is introduced as a data structure $\mathbf{M}$, consisting of several fields:

$$
\mathbf{M}=\left\{\begin{array}{c}
\mathbf{T}^{*} \\
\mathbf{W}_{M} \\
\mathbf{X}_{T R} \\
\mathbf{X}_{V H}
\end{array}\right\} .
$$

The fused model, $\mathbf{M}$ and the camera parameters are then stored in the database and parsed to the deformation estimation method. 

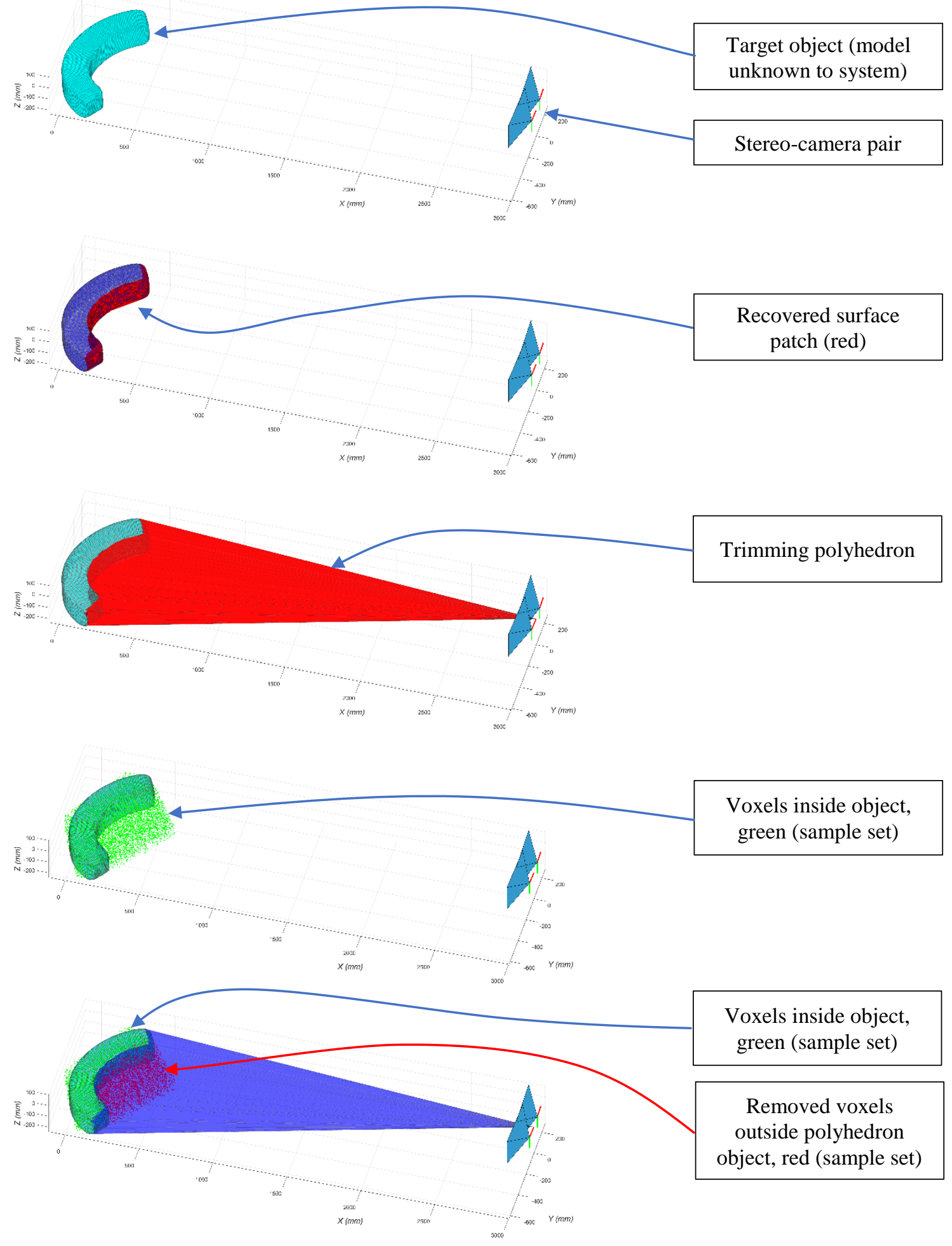

Figure 3.11: Voxel trimming process. 


\section{Chapter 4 \\ Methodology: Deformation Estimation}

The deformation estimation method takes in the recovered, solid object model $\mathbf{M}$ and current camera parameters $\mathbf{P}_{\mathrm{c}}(t)$ from the model generation method and applies an adaptive particle filtering algorithm to estimate the deformation. The output of the deformation estimation is the expected model's deformation, $\mathbf{M}^{+}$, for the next demand instant. The expected deformation model and the camera parameters are parsed to the reconfiguration method.

\subsection{Tracking}

The tracking process implements 3D tracking-through-detection with a user-defined motion model. An unknown object implies an unknown motion model, which may be simple, or complex. To set a standard complexity for the motion model, a constant-acceleration model was chosen for the tracking and prediction process. The benefit of the constant-acceleration model is its applicability to a range of target motions, with limited data requirement, namely, only three data samples are necessary for prediction. Alternative motion models will be discussed at the end of the section.

Tracking implies data association between demand instants. In terms of this work, the object's model must be associated between the current demand instant and the previous demand instant. The tracking-through-detection approach dictates that model features from the currently generated model must be associated with the model generated at the previous demand instant. The visual hull data cannot be explicitly associated between demand instants. In contrast, the SIFT features provide an excellent framework for corresponding features between sampling instants to establish temporal tracking. Further, by corresponding only triangulated SIFT features in both demand instants, tracking can be directly implemented in $3 \mathrm{D}$.

The tracking method is a two-step process: identification of 2D SIFT features that have been matched in more than two demand instants and application of the 3D adaptive particle filter tracker on the triangulated feature. The use of SIFT features allows us to directly estimate the motion of 3D features without the use of 2D trackers such as optical flow, particle swarm tracking, neural net tracking. The complete filtering algorithm is presented in Figure 4.1 and consists of three major streams: Feature Found - the handling of new triangulated points, Feature Tracked - the handling of points that have been located across multiple consecutive demand instants and Feature Lost - the handling points that were previously located but were not located at the current demand instant. Only the Feature Tracked stream outputs data to the global methodology. 


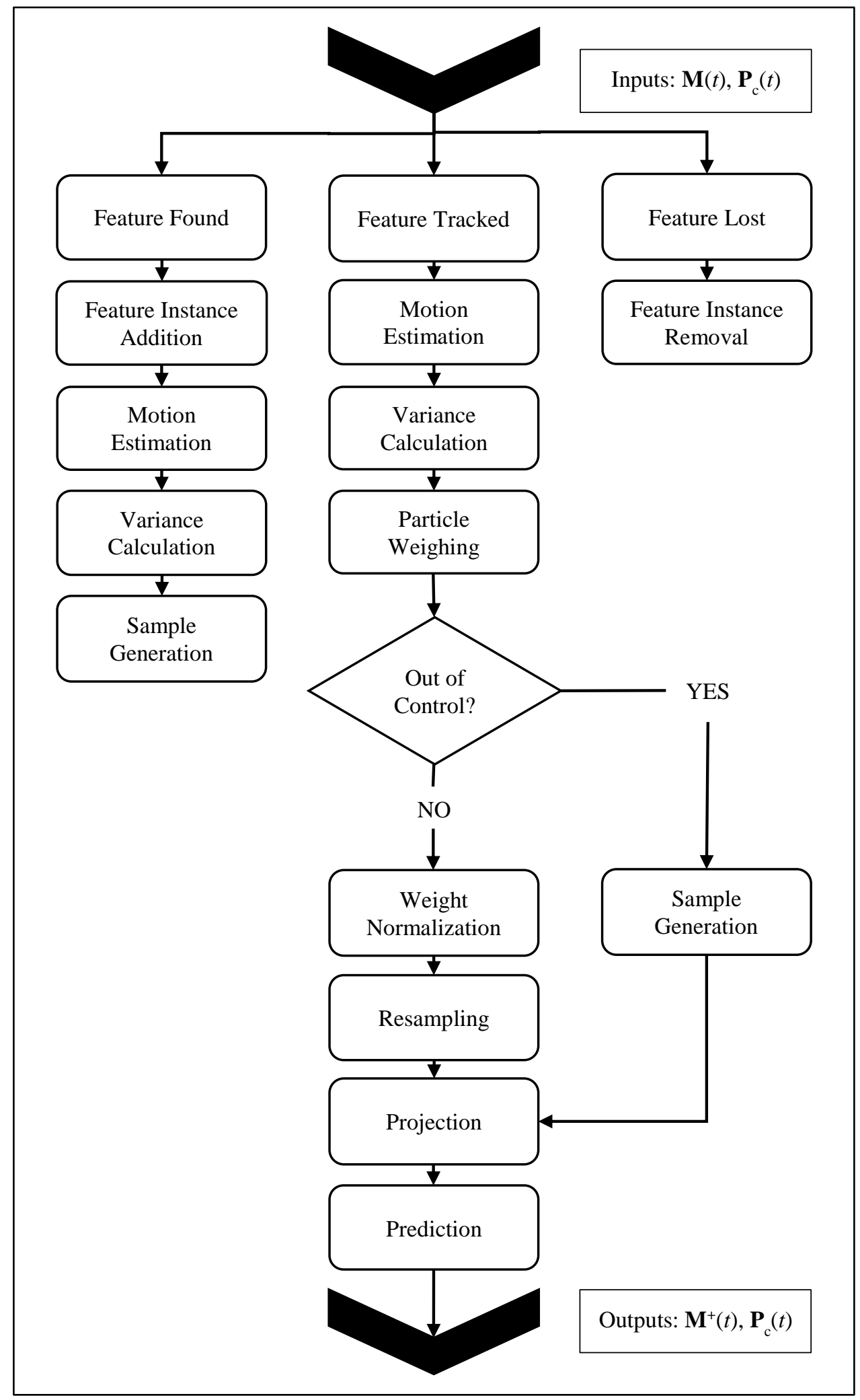

Figure 4.1: Adaptive particle filtering algorithm for deformation estimation. 
The particle filter method was chosen due to its robustness to non-Gaussian noise when compared to Kalman Filter based methods [89], [92]. Furthermore, the particle filtering framework has been the goto method for trajectory tracking in many current methods [175]-[179]. Newer methods for tracking, such as particle swarm optimization [180] and neural nets [181] are indeed available, but the tracking problem could be framed well within the bounds of a particle filter implementation without the need for optimization techniques or network training data.

The on-line adaptive particle filter loads the measurement data from the 3D database for the current and previous demand instants. Each 3D point from the previous demand instant is checked against the current set of 3D points based on the unique key-identifier assigned to them in triangulation to determine whether the point has been tracked across demand instants. Three outcomes are possible: the point could have been tracked across demand instants, the point could have been lost, or the point is new.

Automatic initialization identifies all newly triangulated points and allocates space for the necessary particles for tracking and a state-space measurement history of the last five demand instants. This process works for tracking starting from Demand Instant 0, as all triangulated points will be labeled as new in comparison with other methods that require an a priori demand-instant measurement [30], [158].

\subsubsection{Feature Found Stream}

The feature found stream applies to all points detected in three or less consecutive demand instants. If the feature has only been detected in less than three consecutive instants, its state-space measurement is updated. Features triangulated in three consecutive demand instants have their motion estimated through a $1^{\text {st }}$ and $2^{\text {nd }}$ order backward differencing operation:

$$
\begin{gathered}
\dot{\mathbf{X}}_{T R}(t) \cong \frac{\mathbf{X}_{T R^{*}}(t)-\mathbf{X}_{T R^{*}}(t-\Delta t)}{\Delta t}, \\
\ddot{\mathbf{X}}_{T R}(t) \cong \frac{\mathbf{X}_{T R^{*}}(t)-2 \mathbf{X}_{T R^{*}}(t-\Delta t)+\mathbf{X}_{T R^{*}}(t-2 \Delta t)}{\Delta t^{2}},
\end{gathered}
$$

where $\Delta t$ is the change in time between demand instants, $\mathbf{X}_{\mathrm{TR}}^{\mathrm{T}}(t)$ is the $3 \mathrm{D}$ positional measurement matrix of all tracked points, a subset of all tracked points $\mathbf{X}_{\mathrm{TR}}(t), \dot{\mathbf{X}}_{\mathrm{TR}}$ is their estimated velocity and $\ddot{\mathbf{X}}_{\mathrm{TR}}$ is their estimated acceleration. The constant-acceleration model requires nine total states; three positions, three velocities, and three accelerations.

The total number of particles, $q$, is user-defined. The number of particles used determines the accuracy of the prediction at the cost of computational power [89]. The number of particles may be varied on-line based on the calculation of the total effective number of particles [89], [179]. Herein, the number 
of particles was kept static throughout all demand instants to avoid dynamic memory reallocation tasks. Therefore, each particle filtering instance initialized is composed of a $[9 \times q]$ particle matrix. The particles are drawn from a normal distribution for each tracked point:

$$
\mathbf{Q}_{j}=\mathrm{N}\left(\boldsymbol{x}_{j}^{*}, \boldsymbol{\sigma}_{p_{j}}^{2}\right),
$$

where $\boldsymbol{x}_{j}{ }^{*}$ is the nine-dimensional state-vector of the $j^{\text {th }}$ tracked point and $\boldsymbol{\sigma}_{p_{j}}^{2}$ is its associated variance. The measurement variance, $\sigma_{N_{j}}^{2}$, is set equal to the particle variance, to ensure the particle weighing step also adapts on-line with varying deformation dynamics.

The particle variances are calculated as:

$$
\boldsymbol{\sigma}_{p}^{2}=\frac{1}{2}\left[\begin{array}{l}
\left|\mathbf{X}_{T R}^{T}(t)-\mathbf{X}_{T R}^{T}(t-\Delta t)\right| \\
\left|\dot{\mathbf{X}}_{T R}(t)-\dot{\mathbf{X}}_{T R}(t-\Delta t)\right| \\
\left|\ddot{\mathbf{X}}_{T R}(t)-\ddot{\mathbf{X}}_{T R}(t-\Delta t)\right|
\end{array}\right],
$$

which are updated with every measurement of a tracked point. The on-line updating of the variances ensures the particles remain within close proximity to the measurement. The initial set of particles is, then, generated given the motion model and the particle variance.

The underlying assumption of the sampling distribution is that without an a priori known model of the object, the measured position of the feature is assumed correct within an ellipsoid center about the measurement with a variance of the measurement noise. With each state tracked independently, the result is a dynamically varying ellipsoidal particle distribution proportional to the change in each state and a varying sampling distribution for each particle. The filter implements a recovery process that re-initializes particles if the projected particles are outside of the measurement bounds. This allows the filter the opportunity to remodel the motion dynamics for particles that are not behaving according to the assumed motion.

The particle set is then projected for use in the feature tracked stream. The particle set, $\mathbf{Q}_{j}$, which is projected to determine the expected state-space of the $j^{\text {th }}$ point at the next demand instant:

$$
\mathbf{Q}_{j}^{+}=\mathbf{H} \mathbf{Q}_{j}+\mathbf{U}
$$

where $\mathbf{Q}_{j}{ }^{+}$is the matrix of projected particles for the $j^{\text {th }}$ point, $\mathbf{H}$ is the [9 $\times 9$ ] constant-acceleration state transition matrix, $\mathbf{U}$ is the $[9 \times q]$ uncertainty matrix based on the particle projection variance, Equation (4.4). 


\subsubsection{Feature Tracked Stream}

Points that have been tracked for more than three consecutive demand instants are labeled as tracked and are processed through the feature tracked stream. As a new positional measurement becomes available at the current demand instant, the state-space measurement for a given point is calculated by Equations (4.1) - (4.2) and stored in the state-space measurement history. Thereafter, for each $j^{\text {th }}$ point, the set of projected particles $\mathbf{Q}^{+}{ }_{j}$ is loaded and each nine-dimensional particle is weighed against the current state-space estimate $\boldsymbol{x}_{j}^{*}$ :

$$
\mathbf{W}_{j}=\frac{1}{\sqrt{2 \pi \sigma_{N_{j}}^{2}}} \exp \left(-\frac{\left(\boldsymbol{x}_{j}^{*}-\mathbf{Q}_{j}^{+}\right)^{2}}{2 \boldsymbol{\sigma}_{N_{j}}^{2}}\right)
$$

where $\mathbf{W}_{j}$ is the weight matrix of all particles for the $j^{\text {th }}$ point. The weights are, then, normalized:

$$
\mathbf{W}_{j}^{*}=\frac{\mathbf{W}_{j}}{\sum \mathbf{W}_{j}} .
$$

The normalized weight matrix $\mathbf{W}_{j}^{*}$ is of size $[9 \times q]$. The summed term is checked for a zerocondition which occurs if the projection is too far off from the measured location. All states with a zerosum condition bypass the resampling step and new particles are generated from the most recent measurement. All remaining states with non-zero sums, namely, states with accurate projections, are resampled through a sequential importance process [89]. The regenerated states and resampled states are combined into an updated $\mathbf{Q}_{j}$ matrix.

\subsubsection{Feature Lost Stream}

Points that were previously detected and have been lost at the current demand instant are labelled as lost and are processed through the feature lost stream. The particle matrices and state-space measurement vectors associated with these points are removed from memory, but their unique key-identifiers remain, along with the associated 2D feature keys. Lost points that have been detected anew, in later demand instants, are processed through the feature found stream.

\subsection{Prediction}

The deformation estimation is based on a two-part projection-prediction process within the adaptive particle filtering algorithm. The first part of deformation estimation is the projection of all particle matrices of tracked points, $\mathbf{Q}_{j}$, to produce estimate particle states, $\mathbf{Q}_{j}^{+}$, through Equation (4.5). Then, each tracked points' estimated particle states are averaged to produce the estimated state of each tracked point: 


$$
\boldsymbol{x}_{j}^{*_{+}}=\frac{\sum_{j} \mathbf{Q}_{j}^{+}}{q},
$$

where $\boldsymbol{x}_{j}{ }^{*+}$ is the predicted nine-dimensional state-space of the $j^{\text {th }}$ tracked point. The first three states of all predicted points are placed into the matrix $\mathbf{X}_{T R}^{+}$; the predicted future pose of all tracked, triangulated points.

The visual hull points are not actively tracked between demand instants. The projection of visual hull points from the model $\mathbf{M}$ into the next demand instant requires a velocity and acceleration estimate. These values are estimated from the tracked and triangulated points and the fusion weight matrix $\mathbf{W}_{M}$. Thus, the projection of the visual hull points is:

$$
\mathbf{X}_{V H}^{*}(t+\Delta t)=\mathbf{H}\left[\left[\begin{array}{c}
\mathbf{X}_{V H}(t) \\
\dot{\mathbf{X}}_{T R}(t) \\
\ddot{\mathbf{X}}_{T R}(t)
\end{array}\right] \mathbf{W}_{M}\right]+\mathbf{U}_{T R} \mathbf{W}_{M} .
$$

The result, $\mathbf{X}_{V H}^{*}(t+\Delta t)$, is a $[9 \times m]$ matrix consisting of the projected visual hull points and projected velocities and accelerations, and $\mathbf{U}_{T R}$ is the $[9 \times \mathrm{n}]$ process noise matrix for the tracked, triangulated points. The first three rows of $\mathbf{X}_{V H}^{*}(t+\Delta t)$ are placed into $\mathbf{X}_{V H}^{+}$. Thus, the visual hull portion of the model is the only component of the model used for deformation estimation.

The predicted triangulated points and visual hull points in $\mathbf{X}_{T R}^{+}$and $\mathbf{X}_{V H}^{+}$are recombined with the fused mesh $\mathbf{T}^{*}$ to produce the deformation estimation model $\mathbf{M}^{+}$:

$$
\mathbf{M}^{+}=\left\{\begin{array}{c}
\mathbf{T}^{*} \\
\mathbf{W}_{M} \\
\mathbf{X}_{T R}^{+} \\
\mathbf{X}_{V H}^{+}
\end{array}\right\}
$$

\subsection{Modularity}

The tracking and prediction task allows for modularity in the filtering methodology chosen, including particle filters, KFs [90], EKFs [182], UKFs [91] and PSO [180], [183]. The particle filter was chosen due to its robustness to non-Gaussian noise [89], [92] and its common-place implementation in tracking methodologies [176], [184]. A KF may not necessarily work well since camera noise is non-Gaussian [174] and, thus, tracking may fail. EKFs and UKFs may be better suited than regular KFs for tracking as they do not explicitly depend on Gaussian process noise. PSO should function similarly to particle filtering methods, but would require an optimization step that filtering does not. 
The motion model may be user-selected as well for specific tasks, if necessary. The constantacceleration motion model may be replaced with another simpler or more complex models. A constantvelocity model would decrease the state-space size required and the number of initial tracking demand instants with lower accuracy prediction. A Fourier series model may be used for objects that would undergo cyclic motion [185], [186]. One can note that the motion model selected would dictate the total number of demand instants required before an accurate prediction could be made. For example, a constant-velocity model only requires two demand instants, while a constant-acceleration model requires three. The constantacceleration model was chosen herein as a generalized approach for tracking due to its robustness to capturing human motion and certain non-linear motions. 


\section{Chapter 5 \\ Methodology: Reconfiguration}

This chapter focuses on the reconfiguration methodology developed for the system. Reconfiguration is responsible for proactively repositioning cameras based on the expected model's deformation to improve the shape recovery goals, namely, accuracy and completeness. The accuracy and completeness goals are achieved through the implementation of the modeling methodology within an optimization framework based on maximizing the stereo-visibility of the target objects surface.

\subsection{Overview}

The purpose of the reconfiguration method is to analyze a set of potential new camera parameters, namely, position and orientation, that would improve the surface area visibility of the target object in a dynamic environment. Reconfiguration allows the system of mobile cameras to overcome the object's expected selfocclusions and obstacle occlusions and even poor camera placement, Figure 5.1. The problem definition chapter framed the reconfiguration problem as the maximization of surface area visibility based on the expected object's deformation, Equation (2.5). Herein, the model generation and deformation estimation methods are tied together with the reconfiguration method to produce a single generic shape recovery implementation for a priori unknown, deforming objects in dynamic workspaces.

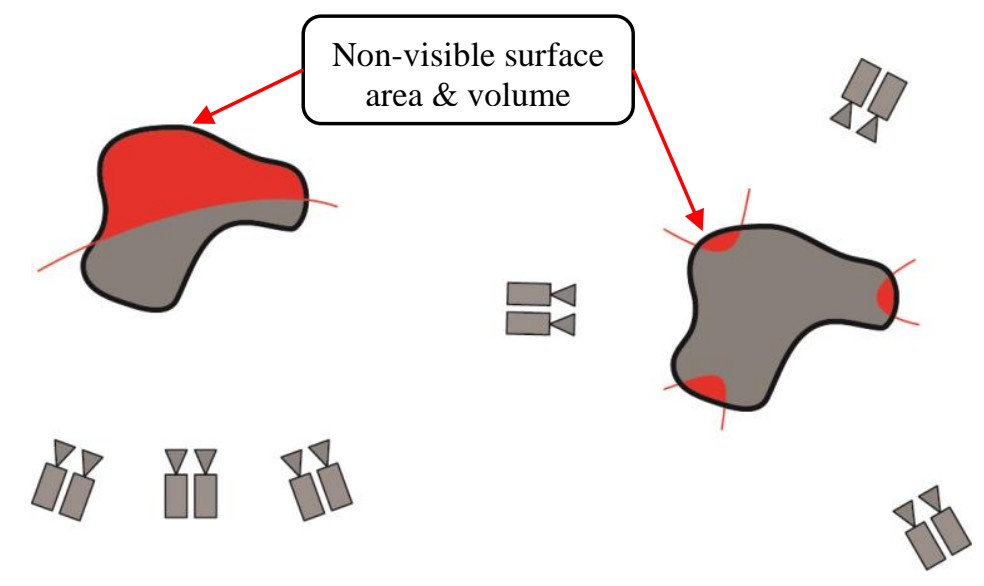

Figure 5.1 Left: poor camera configuration, right: improved camera configuration.

The proposed method consists of a set of processes shown in Figure 5.2. The expected object's deformation model, $\mathbf{M}^{+}(t)$ and the set of all camera parameters, $\mathbf{P}_{\mathrm{c}}(t)$ are parsed from the deformation estimation method. The reconfiguration space is discretized based on the workspace constraints to produce a set of possible camera placement vectors about the generated model. These vectors are generalized based on the selected stereo-camera pairs available in the workspace (Section 3.3). The visibility of each 
placement vector is assessed, producing a unique, binary visibility map for each placement vector. The near-optimal camera placement reconfiguration is chosen as a specific combination of visibility maps such that the total expected surface area visibility is maximized. Finally, given the near-optimal camera poses and the current camera poses, a path is planned for each camera to move towards its next near-optimal pose.

The generalization of the cameras and the visibility objective function reduces the combinatorial problem introduced in Section 2.2.3, Equation (2.6) and Figure 2.6 by combining all camera parameter sets into individual positing vectors. The generation of the positioning vectors accounts for workspace constraints, specifically, obstacle occlusions and camera motion limits. A secondary placement optimization along the positioning vectors ensures stereo-camera placements maximize the object's projection in the FOV. By generalizing the placement vectors, the 2D combinatorial problem in Figure 2.6 is reduced to a single dimension, Figure 5.3.

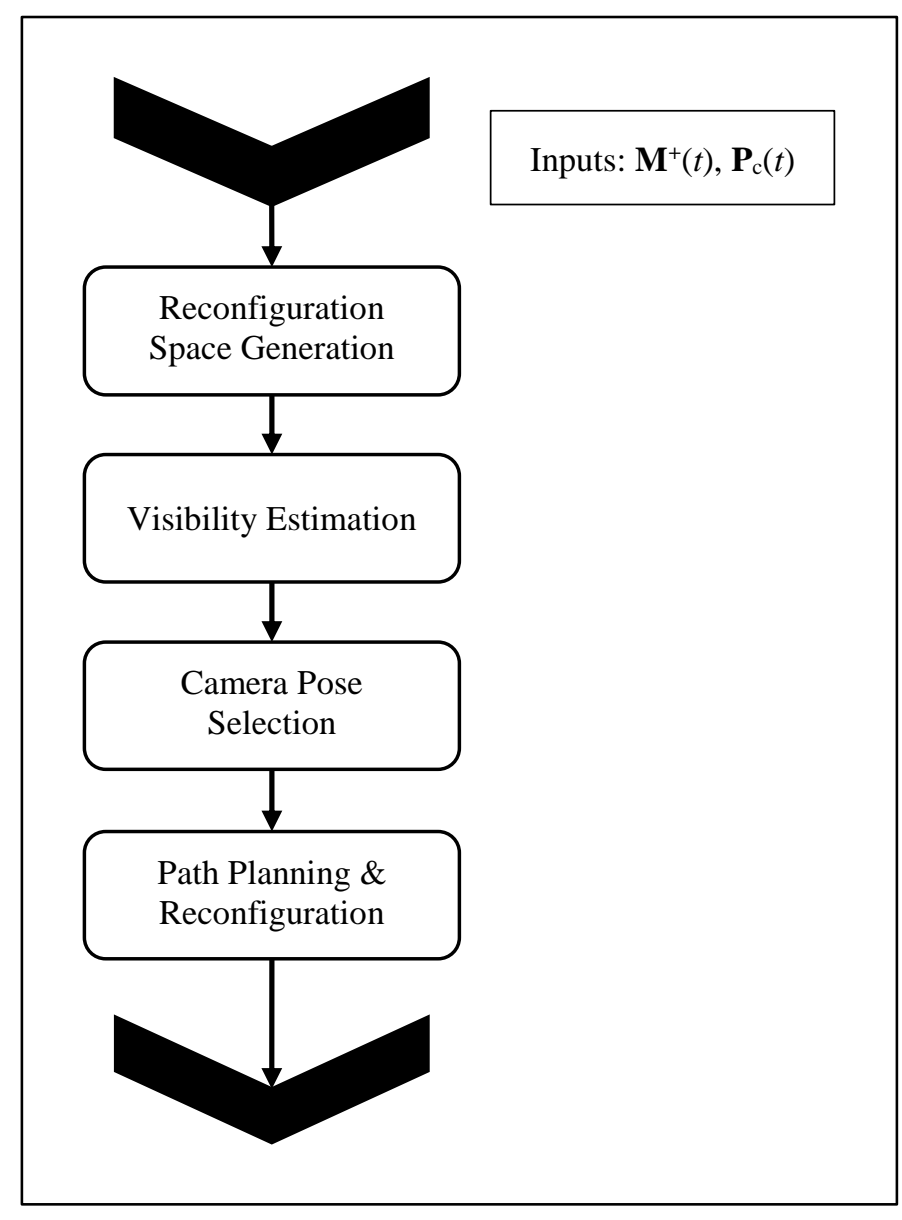

Figure 5.2: Reconfiguration method. 


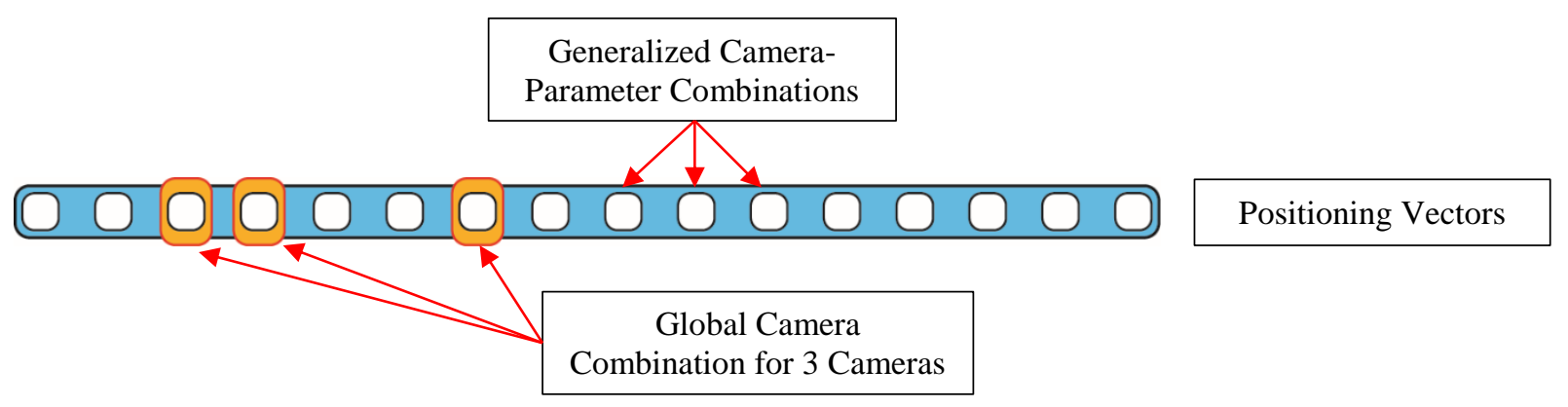

Figure 5.3: Generalized positional combination problem.

\subsection{Reconfiguration-Space Generation}

The reconfiguration space generation is a modular technique that yields a set of test position vectors, $\mathbf{V}$, about the target object's expected model, $\mathbf{M}^{+}$. Ideally, these vectors should be centered about the target object to ensure stereo-camera FOV constraints downstream. In reality, camera placement limitations may not allow centering about the target object. Each, in the matrix $\mathbf{V}$, represents a potential vector along which a stereo camera pair could be placed during reconfiguration. The total number of vectors, $n_{v}$, and the vector distribution method are user-set. Therefore, given $n_{v}$ vectors and $k_{\mathrm{T}}$ stereo camera pairs, the total number of possible positional combinations, $p_{\max }$, is calculated as:

$$
p_{\max }=\frac{n_{v} !}{k_{T} !\left(n_{v}-k_{T}\right) !} .
$$

Each positional combination is defined by the indexing matrix $\mathbf{R}$, a $\left[p_{\max } \times k_{\mathrm{T}}\right]$ integer matrix whose columns denote a stereo camera pair and each row indexes a potential positioning vector. Under certain circumstances, many combinations in $\mathbf{R}$ are non-unique as the angular separations between the vectors are identical, but the orientation of the vectors relative the object's center is rotated (i.e., the $i^{\text {th }}$ and $j^{\text {th }}$ vector combinations in Figure 5.4 are related by a single rotation). A non-generalized approach to placement vector generation would result in the problem described by Figure 2.6, with an exponential number of positional combinations for all cameras. The proposed generalized approach reduces the total number of positional combinations per Figure 5.5, on a logarithmic scale, indicating the clear advantage of generalization. 


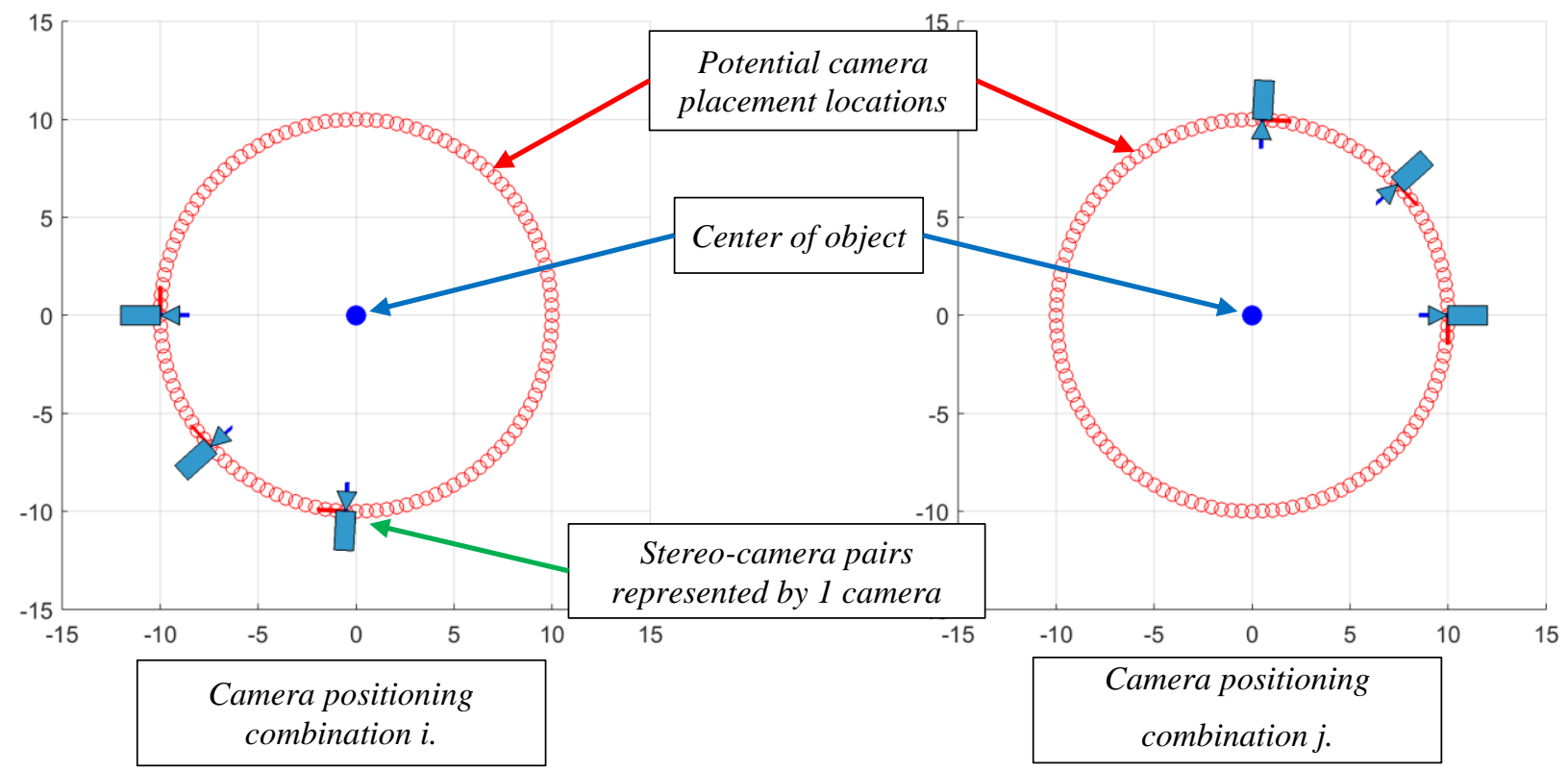

Figure 5.4: Example of a pseudo-redundant combination.

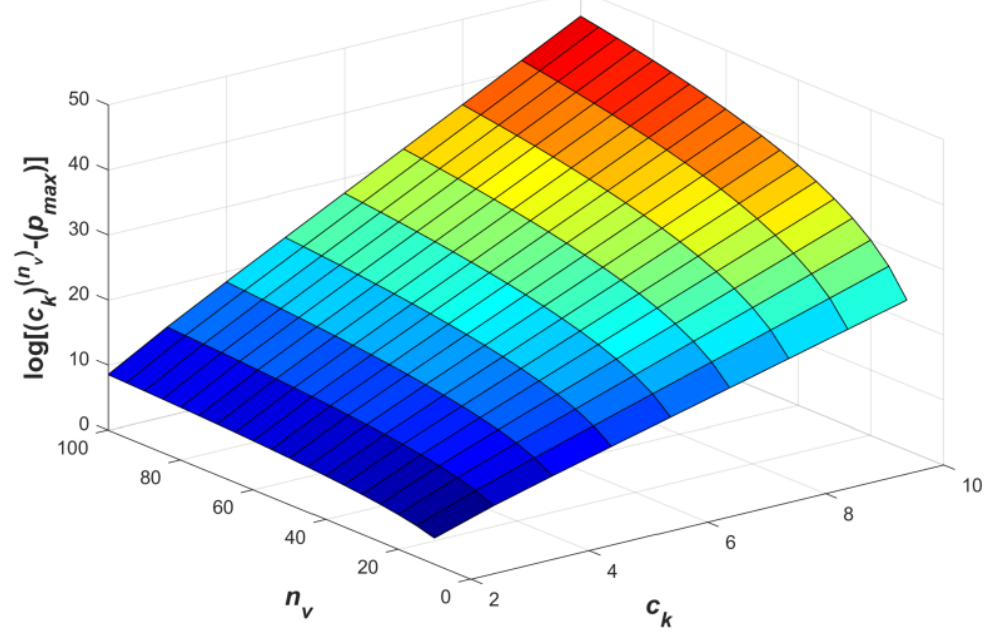

Figure 5.5: Logarithm of the difference between the total number of positional combinations for independent placement vectors and generalized vectors.

Although it would be possible to eliminate such pseudo-redundant combinations and replace them with a rotational relation, this could lead to the over-complication of positional vector generation. Specifically, if choosing a spherical coordinate system to generate positional vectors, certain combinations may be automatically generated by rotating the vectors (due to redundancy), but such a redundancy may not exist with a different vector-generation scheme, such as box-surface points, hemispherical coordinates, 
or even circumferential coordinates. Thus, these pseudo-redundant combinations are not eliminated in the combination generation. An important consideration of the vector space generation is that the vector distribution must be uniform about the target object's expected deformation to ensure that positional combinations are not spatially biased. For example, a spherical coordinate system can be used to generate the positional vectors about the target object, but standard $r-\theta$ increments cannot be used Figure 5.6.

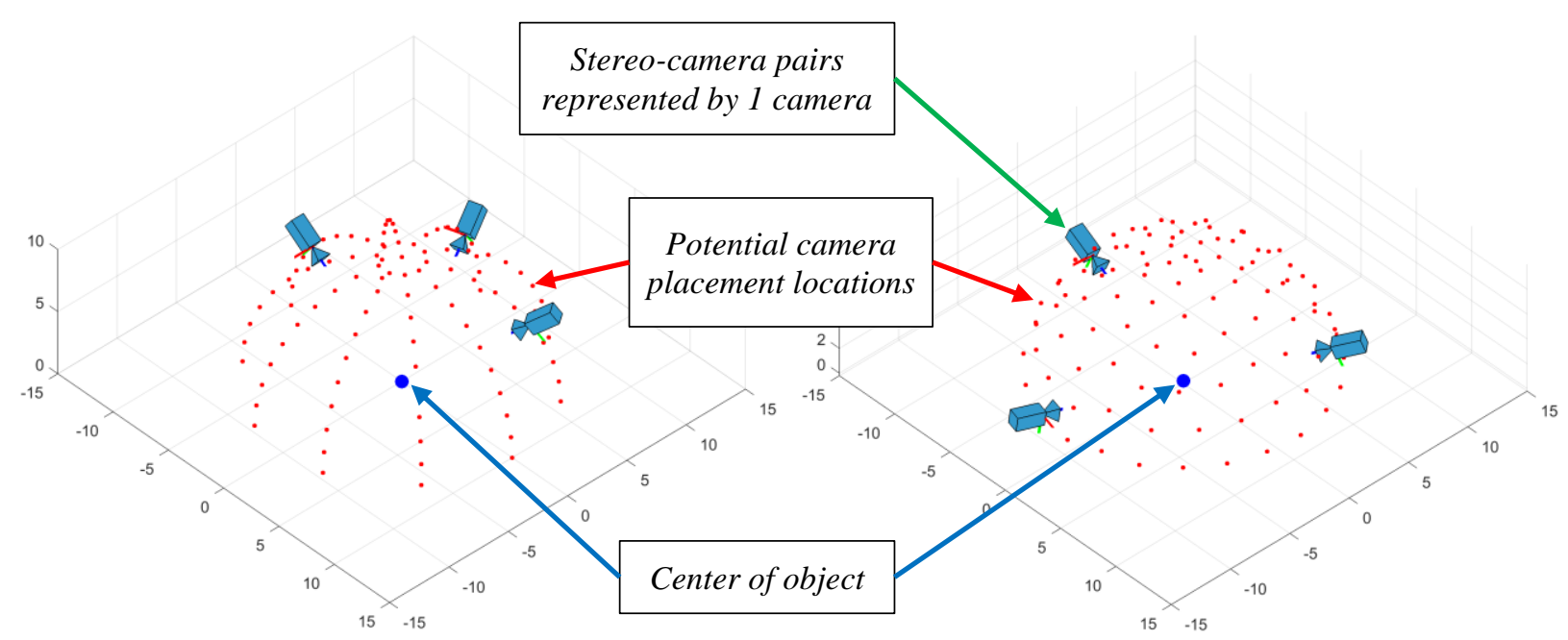

Figure 5.6: Spherical vector space, left: standard $r-\theta$ increments, right: uniformly distributed vectors from non-standard $r-\theta$ values.

There exists a theoretical computational limit on the number of positioning vectors used that depends on the total number of stereo camera pairs. Namely, as the number of vectors increases, the total number of possible positional combinations increases faster than $O\left(c^{n}\right)$. Thus, the total number of operations and storage requirements increase with the total number of combinations. For example, Figure $5.7(a)$ represents the data storage limitation imposed in MatLab when compared to the total number of combinations possible for a variable number of stereo camera pairs. It is evident that past five pairs, an alternative reconfiguration space generation method would be required that massively reduces the number of potential positioning vectors. Figure $5.7(b)$ illustrates the total number of combinations generated that must then be processed.

The total number of combinations can be reduced by eliminating ill-positioned ones. These are defined as combinations that have at least one angle between a pair of vectors less than a user-defined threshold, $\theta_{p-\min }$. Intuitively, these combinations have positioning vectors in the same spherical quadrant that would lead to surface area visibility overlap, Figure 5.8. For example, the total number of combinations 
can be reduced by about $43 \%$ for three positioning vectors with a minimum threshold of $30^{\circ}$, if the reconfiguration space is circumferential on a common plane (as in Figure 5.8). The reduction rate, $\eta_{r}$, is calculated as:

$$
\eta_{r}=1-\frac{p_{\max }^{*}}{p_{\max }}
$$

where $p_{\max }^{*}$ is the number of positional combinations after eliminating the ill-positioned vector combinations.
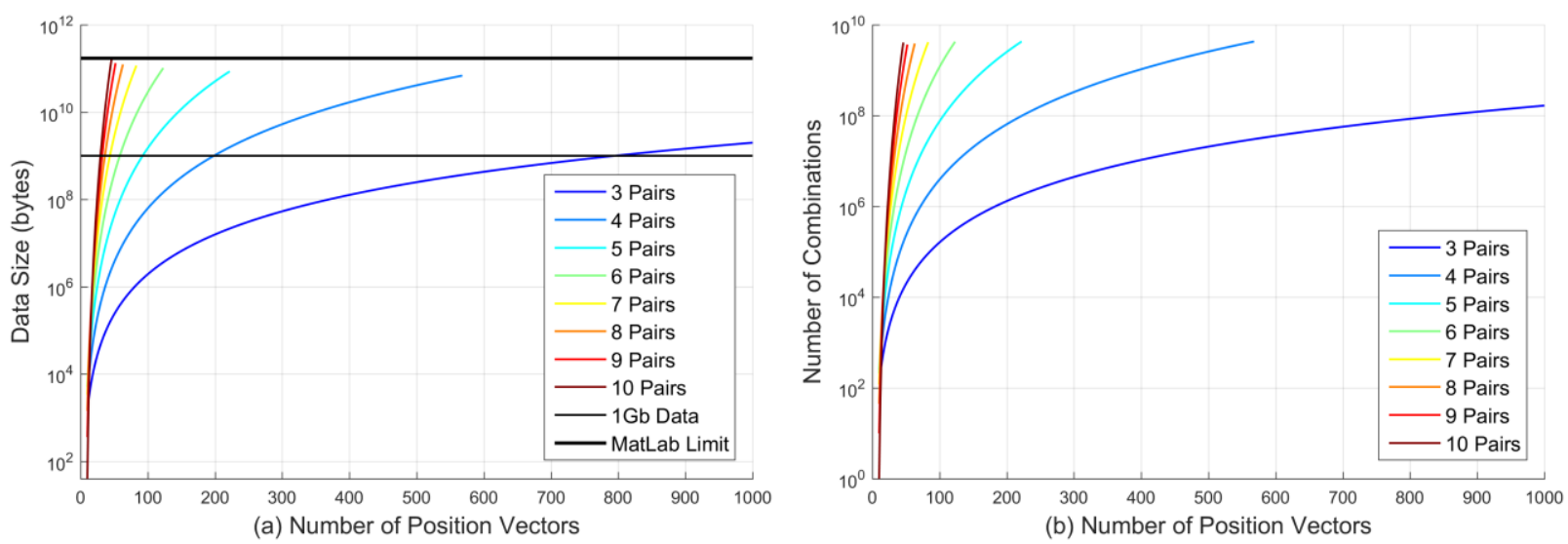

Figure 5.7: (a) Data storage requirements for index matrix $\mathbf{R}$, when varying the number of positions for different numbers of camera pairs; $(b)$ the total number of combinations.

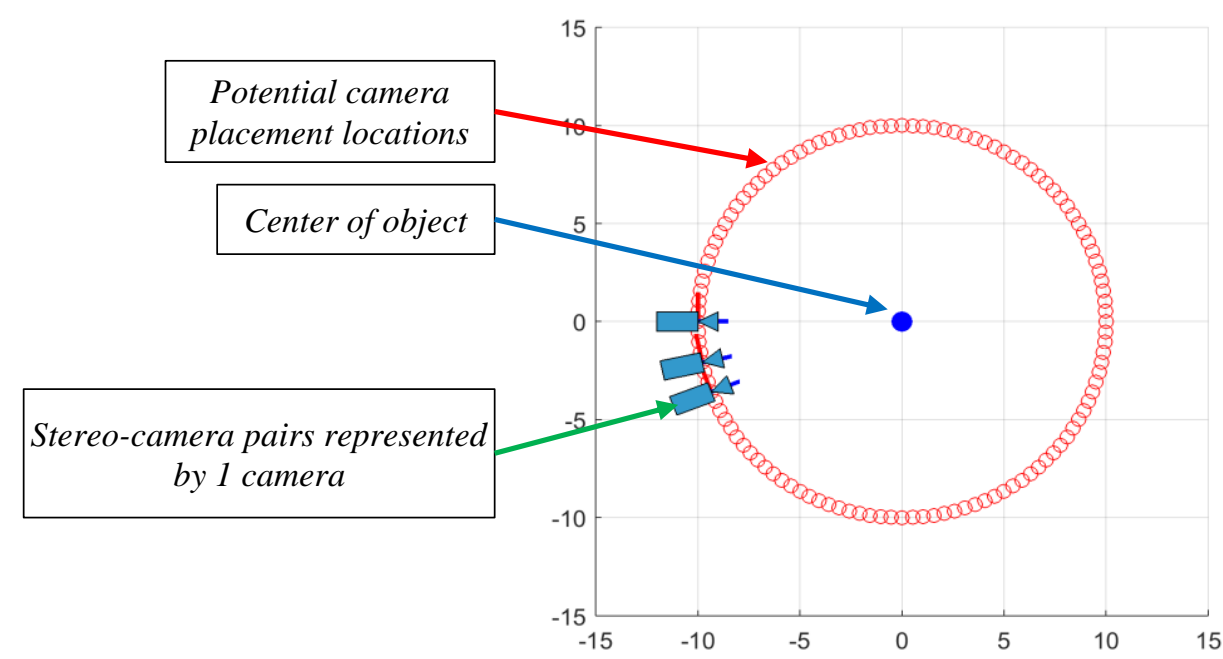

Figure 5.8: Example of ill-positioned vector combination, wherein the three stereo-camera pairs are too close. 
The reconfiguration space generation process yields two matrices, the set of positional vectors, $\mathbf{V}$ and the final reduced matrix of combinational indices, $\mathbf{R}^{*}$. This process is dynamic as the positional vectors and combinational index matrix are regenerated at each demand instant based on the number of stereo camera pairs available. This makes the method robust to the varying number of stereo camera pairs.

\subsection{Visibility Estimation}

Visibility estimation is a generalized function that may be applicable to different types of camera configurations. Once the reconfiguration space generation process determines a set of positional vectors, the visibility-estimation process must determine a binary visibility map for each vector. The $\left[n_{p} \times n_{v}\right]$ visibility map matrix, $\mathbf{V}_{\text {map }}$, represents the estimated visible surface area proportion each polygon in the model. Each row of $\mathbf{V}_{\text {map }}$ represents a model's polygon and each column represents one possible positional vector. The estimated visibility for each positional vector is calculated independently and can be parallelized. The purpose of calculating the visibility for each positioning vector individually, instead of calculating the cumulative visibility for a positional combination, is simple: there will always be less positioning vectors than possible combinations, thus, reducing the computation workload by a factorial factor.

The individual visibility-estimation process for a given positional vector requires three inputs: the expected model deformation, $\mathbf{M}^{+}$(polygon model with normals), a unit test position vector, $\boldsymbol{v}_{\text {test }}$ and the expected obstacle pose, $\mathbf{O}^{+}$(where the obstacle is modelled as a triangle-mesh object). A point, $\boldsymbol{p}^{*}$, is chosen along the vector $\boldsymbol{v}_{\text {test }}$ at a length of at least the norm of the model's Cartesian range (in $x$-, $y$ - and $z$-axes), centered about the model $\mathbf{M}^{+}$. This length ensures the visibility estimate places the stereo camera pairs sufficiently far away from the target object, without compromising a field-of-view constraint. The model $\mathbf{M}^{+}$is, then, converted into a collection of polygon vertices and polygon centers to produce a single matrix, $\mathbf{X}_{\text {test }}[k \times 3]$, of test points.

Once the inputs have been parsed, five distinct visibility operators are applied to determine the visibility of each of the model's polygons: ( $i$ ) obstacle logical gate, (ii) hidden-point removal (HPR) [187], (iii) normal rejection, (iv) image width decay and (v) depth-of-field decay. The width and depth operators were added as 'optional' to be used at the user's discretion. In order to generalize the application of the visibility function, each camera pair is modelled as a single depth sensor placed along the test positional vector. This simplification removes the operators' dependence on specific stereo parameters and allows a more general application of the function across varying stereo baselines and may even be implemented for projection (active) camera sensors. The visibility-estimation process is outlined in Figure 5.9. 


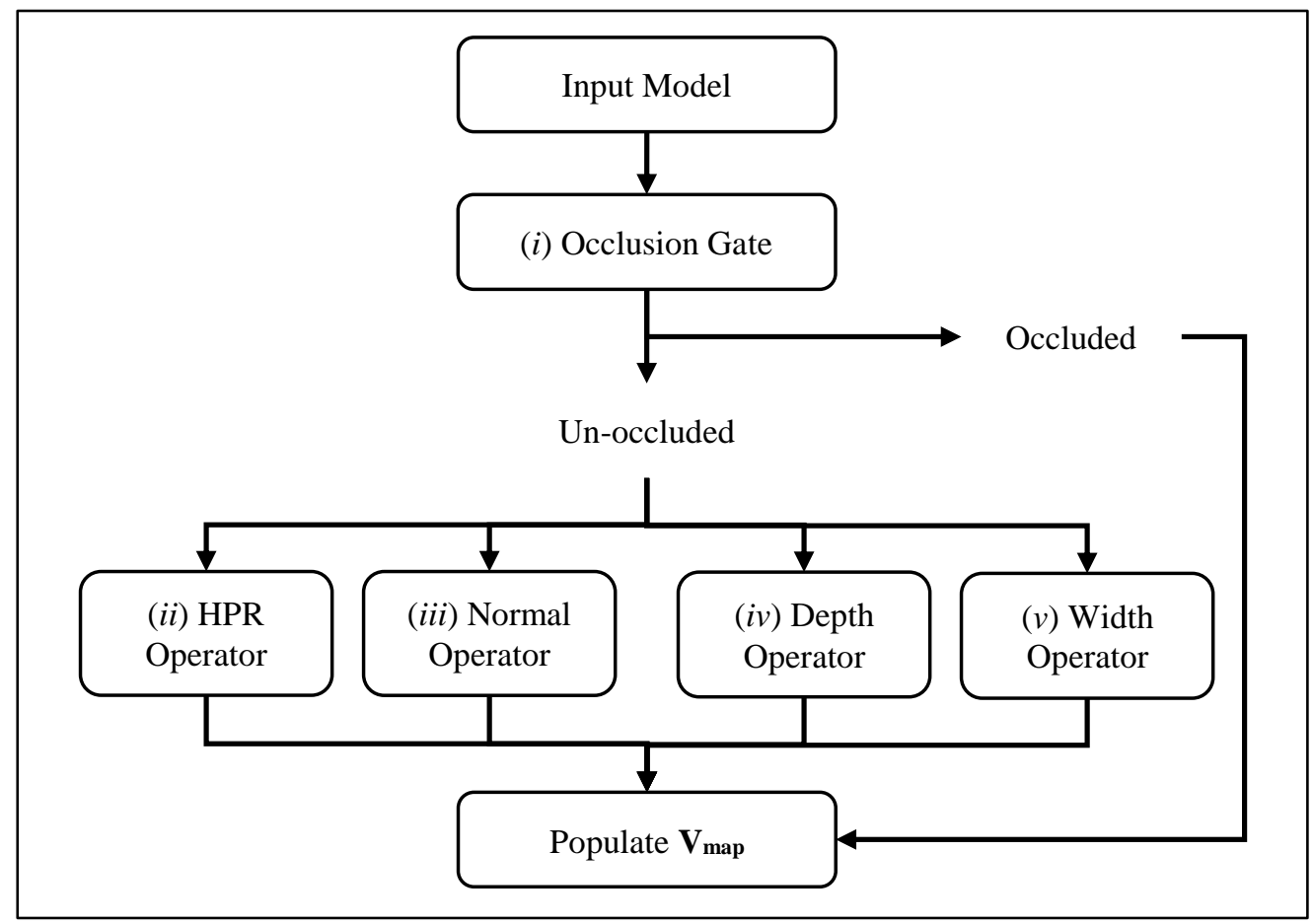

Figure 5.9: Visibility-estimation process outline.

The obstacle logical gate is the first operation of the visibility-estimation process. The gate checks whether any test points are occluded for a given test position vector using a single ray-casting operation [188]. The occlusion check begins by projecting the 3D obstacle model onto a plane defined by the center of the obstacle, $\boldsymbol{c}_{o b s}$, and a vector between $\boldsymbol{c}_{o b s}$ and $\boldsymbol{p}^{*}$. The outer boundary of the flattened obstacle mesh is used to create a $2 \mathrm{D}$ polygonal region representing the obstacle's silhouette. The ray-triangle intersection algorithm in [188] is, then, used to check whether the line segments between $\boldsymbol{p}^{*}$ and each point in $\mathbf{X}_{\text {test }}$ intersect the 2D obstacle silhouette. Any intersection results in a zero-visibility label, namely, a column of zeros in the $\mathbf{V}_{\text {map }}$ matrix, for the current test position vector. Furthermore, an obstacle occlusion bypasses the rest of the visibility operators. If no obstacle occlusion exists, the rest of the visibility operators are processed.

The HPR operator estimates the test points that are self-occluded without a processor-heavy raytracing algorithm. The HPR operator spherically projects all the test points and selects all the points lying on the convex hull generated from the projection as visible. The radius for the spherical projection is set to the maximum distance between the point, $\boldsymbol{p}^{*}$ and all the test points in the model. The HPR operator stores the Boolean visibility values for each test point into a vector $\boldsymbol{v}_{H P R}$. 
The normal rejection operator checks the angular separation between each test point normal and the expected optical axis represented by the position vector. The angular separation, $\phi$, is calculated as the inverse cosine of the dot product between the position vector and each normal:

$$
\varphi_{i}=\cos ^{-1}\left(\boldsymbol{v}_{\text {test }} \bullet \boldsymbol{q}_{i}\right)
$$

where $\boldsymbol{q}_{i}$ is the normal of the $i^{\text {th }}$ model test point.

Test point normals with separation angles greater than a user-defined threshold $\phi_{\max }$ are set to 'false' in the Boolean vector $\boldsymbol{v}_{\text {norm }}$. The strength of this operator is apparent in systems with a short focal length that experience a foreshortening effect [189], as surfaces tilting further away from the stereo-pair will be labelled as non-visible.

The width decay operator is a supplementary filter based on the likelihood that unique, 2D, surface features will be accurately corresponded and triangulated around the outer edges of a given stereo camera pair's images. The operator projects all test points in $\mathbf{X}_{\text {test }}$ onto a plane defined by the center of the model $\mathbf{M}^{+}$and the position vector $v$ :

$$
\mathbf{X}_{\text {test }}^{*}=\mathbf{X}_{\text {test }}-\left[\left(\mathbf{X}_{\text {test }}-\mathbf{C}_{M}\right) \boldsymbol{v}_{\text {test }}^{T}\right] \boldsymbol{v}_{\text {test }},
$$

where $\mathbf{C}_{M}$ is the center of the model $\mathbf{M}^{+}$repeated for $k$ test points, $[k \times 3]$ and $\boldsymbol{v}_{\text {test }}$ is a $[1 \times 3]$ unit vector. The resulting points, $\mathbf{X}^{*}$ test, are located on the plane defined by the model's center point $\boldsymbol{c}_{M}$ and the test position vector. The distance from the model center to each projected test point is calculated and stored in the variable $\boldsymbol{d}^{w}$. Then, each test point distance is compared to the distance threshold value, $d_{t h r e s h}^{w}$ :

$$
d_{\text {thresh }}^{w}=\frac{1}{k} \sum_{i=1}^{k} d_{i}^{w}-\sigma_{\text {thresh }}^{w}\left(\sqrt{\frac{1}{k} \sum_{i=1}^{k}\left[d_{i}^{w}-\frac{1}{k} \sum_{i=1}^{k} d_{i}^{w}\right]^{2}}\right),
$$

where $\sigma_{t h r e s h}^{w}$ is the user-defined standard deviation threshold. A Boolean vector, $\boldsymbol{v}_{\text {width }}$, is composed of true values for each test point whose planar distance is below the threshold.

The depth-of-field decay operator is another supplementary filter that is based on the likelihood that 3D features will be detected depending on their relative distance to the object's center along the test position vector. The purpose of this operator is to add penalty onto points triangulated too far from the center of object, as they are more likely to be erroneous [190]. The operator measures the distances from each test point to the model center along the vector $\boldsymbol{v}_{\text {test }}$ and stores the distance in the vector $\boldsymbol{d}^{d}$ :

$$
\boldsymbol{d}^{d}=\left(\mathbf{X}_{\text {test }}-\mathbf{C}_{M}\right) \boldsymbol{v}_{\text {test }}{ }^{T}
$$


The distance values are checked against a depth threshold, $d_{t h r e s h}^{d}$ :

$$
d_{\text {thresh }}^{d}=\frac{1}{k} \sum_{i=1}^{k} d_{i}^{d}-\sigma_{\text {thresh }}^{d}\left(\sqrt{\frac{1}{k} \sum_{i=1}^{k}\left[d_{i}^{d}-\frac{1}{k} \sum_{i=1}^{k} d_{i}^{d}\right]^{2}}\right) \text {, }
$$

where $\sigma_{\text {thresh }}^{d}$ is the user-defined standard deviation threshold for the relative distance between each test point and a plane defined by the object's center and the test position vector. The threshold should be set according to the expected depth of field of the stereo camera pair. A Boolean vector, $\boldsymbol{v}_{\text {depth }}$, is composed of true values for each test point's planar distance below the threshold.

The Boolean vectors from each operator are concatenated into a single logical matrix $\mathbf{Y}[k \times 4]$. Each operator could be turned on or off within the process depending on the a priori knowledge available for each stereo camera pair. Namely, it may be desirable to disable depth decay for general systems using mixed camera cases. The matrix $\mathbf{Y}$ is, then, converted to a visibility vector $\boldsymbol{y}$ through a logical conjunction across the rows, wherein for a test point to have a true visibility value in the vector $\boldsymbol{y}$, it must have been visible by the four operators.

The vector $\boldsymbol{y}$ is split to represent the vertices and the polygon centers and each polygon's visibility is assessed by a look-up, Table 5.1. Using this table, a final proportional visibility vector is computed from $\boldsymbol{y}$ and placed into the corresponding column of $\mathbf{V}_{\text {map. }}$.

The output of the visibility-estimation process is the matrix $\mathbf{V}_{\text {map }}$ that represents the estimated proportional visibility of each polygon in the object model for a given positioning vector. $\mathbf{V}_{\text {map }}$ can, then, be used to determine the combination of stereo camera pair poses that maximize the overall surface visibility of the expected object's deformation.

Table 5.1: Visibility case look-up.

\begin{tabular}{|c|c|c|c|}
\hline $\begin{array}{c}\text { Visible } \\
\text { Vertices }\end{array}$ & $\begin{array}{c}\text { Visible } \\
\text { Center }\end{array}$ & $\begin{array}{c}\text { Visibility } \\
\text { Case ID }\end{array}$ & $\begin{array}{c}\text { Polygon } \\
\text { Visibility Estimate }\end{array}$ \\
\hline $\mathbf{3}$ & $\mathbf{1}$ & 14 & $12 / 12$ \\
\hline $\mathbf{2}$ & $\mathbf{1}$ & 13 & $8 / 12$ \\
\hline $\mathbf{2}$ & $\mathbf{0}$ & 3 & $6 / 12$ \\
\hline $\mathbf{1}$ & $\mathbf{1}$ & 12 & $4 / 12$ \\
\hline $\mathbf{1}$ & $\mathbf{0}$ & 2 & $3 / 12$ \\
\hline $\mathbf{3}$ & $\mathbf{0}$ & 4 & $0 / 12$ \\
\hline $\mathbf{0}$ & $\mathbf{0}$ & 1 & $0 / 12$ \\
\hline
\end{tabular}




\subsection{Camera-Pose Selection}

The optimal camera-pose-selection process is tasked with choosing the positional combination of the stereo camera pairs that maximizes the target's surface visibility, Figure 5.10. First, a score is assigned to each possible positional combination in $\mathbf{R}^{*}$ calculated by taking the indices of positional vectors in $\mathbf{R}^{*}$ for a given combination, $\boldsymbol{r}^{*}$. The indices are, then, used to extract the corresponding columns from $\mathbf{V}_{\text {map }}$ into a smaller temporary matrix $\mathbf{V}^{*}$ map, $\left[n_{p} \times k_{T}\right]$. The maximum values for each row are then summed to produce a score for the given combination:

$$
R(k)=\sum_{i=1}^{n_{p}}\left(\max _{j=1}^{c_{n}}\left[\mathbf{V}_{\text {map }}^{*}(i, j)\right]\right)
$$

where $R(k)$ is the score for the $k^{\text {th }}$ combination of poses.

The score for each combination represents the sum of the proportions of each polygon's expected stereo visibility. Thus, by choosing the positional combination that maximizes the score, the expected visibility of the object at the next demand instant is maximized, as described in Equation (2.5):

$$
\max (R(k)) \Rightarrow \max _{\mathbf{P}_{c}(t+1)}(\Gamma(t+1))
$$

Once the score for each positional combination has been calculated, the optimal camera-poseselection process begins. First, the highest scoring combination is chosen, as it represents the positional combination that is expected to maximize the total proportion of visible polygons on the model. Next, using pre-set or user-defined parameters for stereo-pair positioning (i.e., fixed, or floating stereo baseline and optical axes angles), the process generates a list of test poses along the positional vector for the stereo-pair. The test poses vary the distance of the stereo-pair along the vector and the angular rotation of the pair about the positional vector, Figure 5.11, where $\boldsymbol{o}_{c}$ is the center point between a stereo camera pair. The distance increments and rotation increments are user-defined. The purpose of these increments is to locate the distance along the position vector and rotation about the vector, such that the object's projection is maximized in the field-of-view of both cameras of the stereo-pair.

The maximum projection of the object in both cameras' frames is chosen through a projection fit score, $h$, assigned to each distance and rotation increment. The score is calculated by firstly projecting the expected model deformation into each camera of the pair at a given distance and rotation increment. Then, a bounding rectangle is fit to both projections and the distances between each rectangle edge and image boundary, $g$, are calculated in pixels, Figure 5.12. The score, $h$, is calculated as: 


$$
h=\left(\frac{1}{2}\left(\sqrt{\frac{1}{4} \sum_{i=1}^{4}\left[g_{i}^{L}-\frac{1}{4} \sum_{i=1}^{4} g_{i}^{L}\right]^{2}}+\sqrt{\frac{1}{4} \sum_{i=1}^{4}\left[g_{i}^{R}-\frac{1}{4} \sum_{i=1}^{4} g_{i}^{R}\right]^{2}}\right)\right)^{-1},
$$

where $g_{i}{ }_{i}$ is the padding in pixels of the left image for the $i^{\text {th }}$ border (top, bottom, left, or right) and $g_{i}^{R}$ is the padding in pixels of the right image. The score represents the mean deviations of padding values for both images.

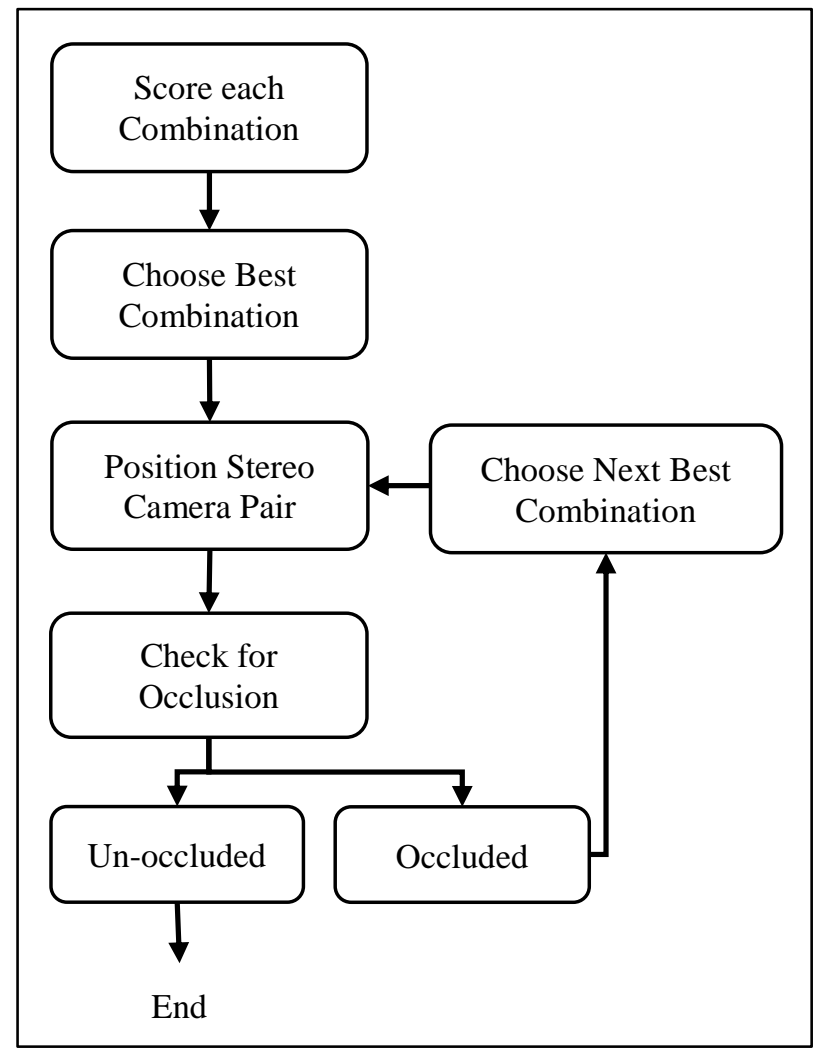

Figure 5.10: Optimal pose selection process.

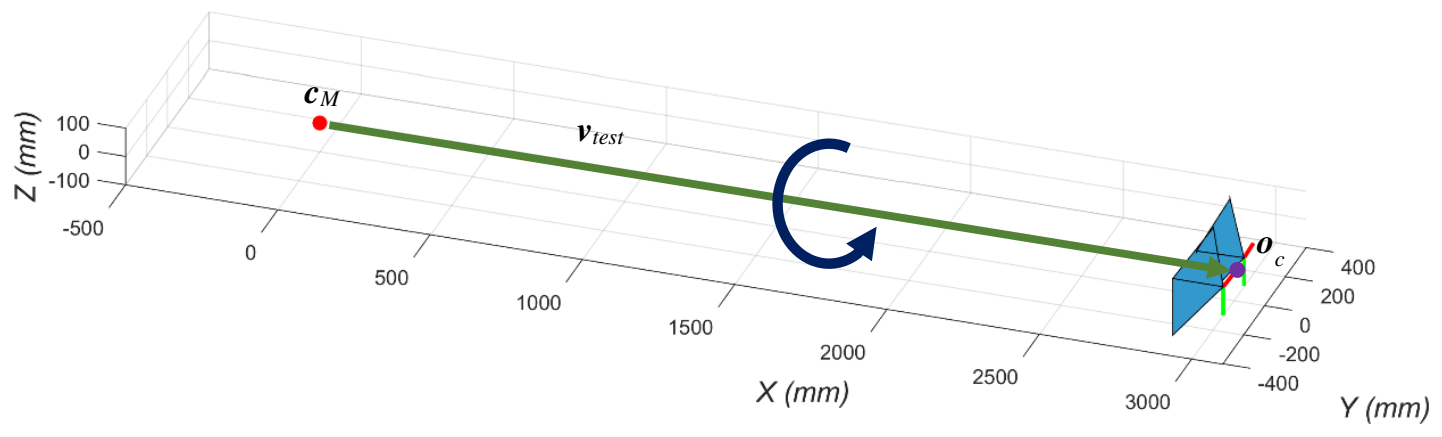

Figure 5.11: Overview of positional and rotational stereo-pair placement for a given positioning vector. 
Quantitatively, the maximum scoring distance and rotation increment places the stereo-pair such that the expected projection of the object is maximized in both fields of view with equivalent padding around the projection.

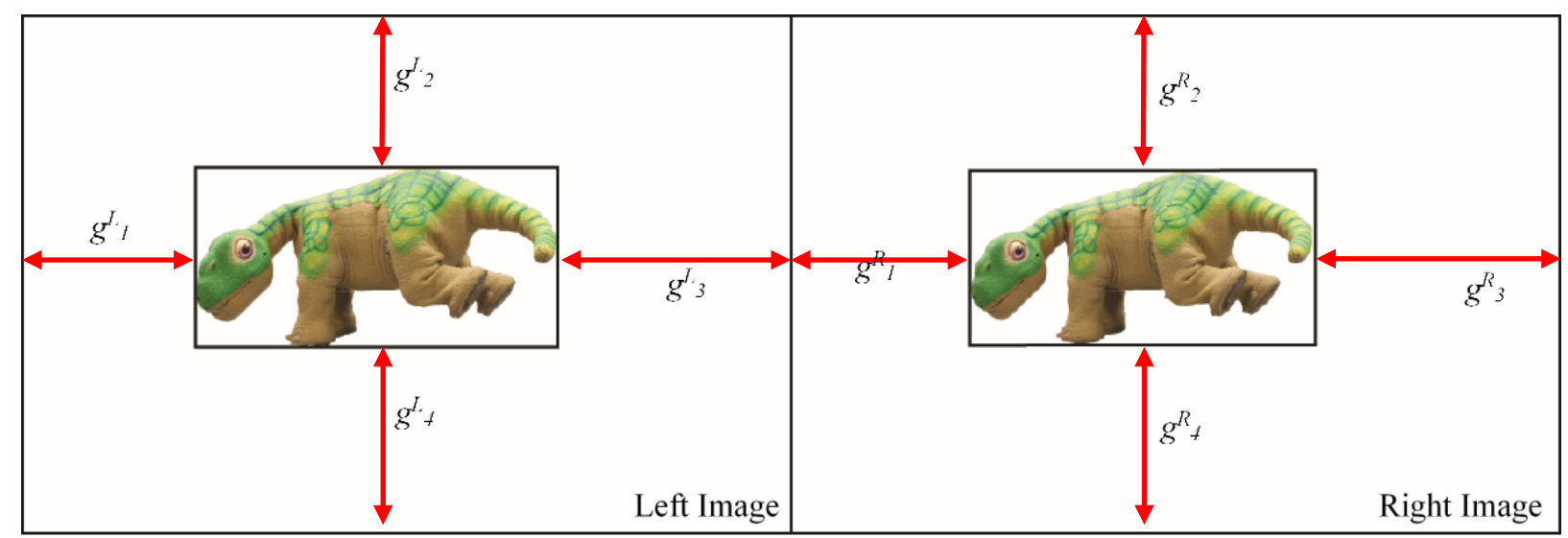

Figure 5.12: Image padding.

Once all stereo-pairs have been placed along their respective positioning vectors, an occlusioncheck ensures that all vertices of the expected model, $\mathbf{M}^{+}$, are visible. This occlusion-check ensures that the positioning vectors and final stereo-pair placement do not result in possible obstacle occlusion. If there exists an expected obstacle occlusion that was not detected in the visibility estimation process (due to its stereo-pair generalization), the current positional combination is removed, the next best combination is chosen and the placement is recalculated. This process is repeated until no obstacle occlusions are detected. This feedback mechanism improves the general application of the proposed method by removing dependence on the visibility function of explicit stereo-pair parameters. When the near-optimal camera placement is complete, the individual camera pair trajectories must be planned.

\subsection{Camera Path Planning}

The camera path planning process maps the current stereo-pairs to the near-optimal positions and, then, generates a motion path. Mapping determines the association between the current stereo-pairs and the nearoptimal stereo-pairs that minimize the path distance. Each association is determined by first calculating the Euclidean distances between all unmapped current stereo-pair poses and near-optimal stereo-pair pose (the poses represent the center point between stereo-pairs, i.e., Point $\boldsymbol{o}_{c}$ in Figure 5.13). The current and nearoptimal pairs with the minimum distance are, then chosen, for association and added to the map. This ensures that the current stereo-pairs are always mapped to the closest near-optimal stereo-pair. 
The path planner fits a Bézier curve between the current mean stereo camera pair's pose, $\boldsymbol{o}_{c}$ and the mean stereo camera pair's pose at the next demand instant, $\boldsymbol{o}_{c}^{+}$, Figure 5.13. Points $\boldsymbol{c}_{M}$ and $\boldsymbol{c}_{M}^{+}$represent the center point of the object at the current and next demand instants, respectively. The point $\boldsymbol{c}_{M}^{*}$ is the triangulation of the vectors $\boldsymbol{c}_{M} \boldsymbol{o}_{c}$ and $\boldsymbol{c}_{M}^{+} \boldsymbol{o}_{c}^{+}$. The Bézier curve is defined by the points $\left\{\boldsymbol{o}_{c}, \boldsymbol{p}_{b}^{l}, \boldsymbol{p}_{b}^{2}, \boldsymbol{o}_{c}^{+}\right\}$, wherein the points $\left\{\boldsymbol{p}_{b}^{l}, \boldsymbol{p}_{b}^{2},\right\}$ are defined such that the vectors pairs $\left\{\boldsymbol{c}_{M} \boldsymbol{o}_{c}, \boldsymbol{o}_{c} \boldsymbol{p}_{b}^{l}\right\}$ and $\left\{\boldsymbol{p}_{b}^{2} \boldsymbol{o}_{c}^{+}, \boldsymbol{c}_{M}^{+} \boldsymbol{o}_{c}^{+}\right\}$are perpendicular and the vector lengths of $\left\{\boldsymbol{o}_{c} \boldsymbol{p}_{b,}^{l} \boldsymbol{p}_{b}^{l} \boldsymbol{p}_{b,}^{2} \boldsymbol{p}_{b}^{2} \boldsymbol{o}_{c}^{+}\right\}$are equal. The curve is located on a plane defined by the points $\left\{\boldsymbol{o}_{c}, \boldsymbol{o}_{c}^{+}, \boldsymbol{c}_{M}^{*}\right\}$. Point $\boldsymbol{o}_{c}^{*}$ is the stereo camera pair placement location for the next demand instant.

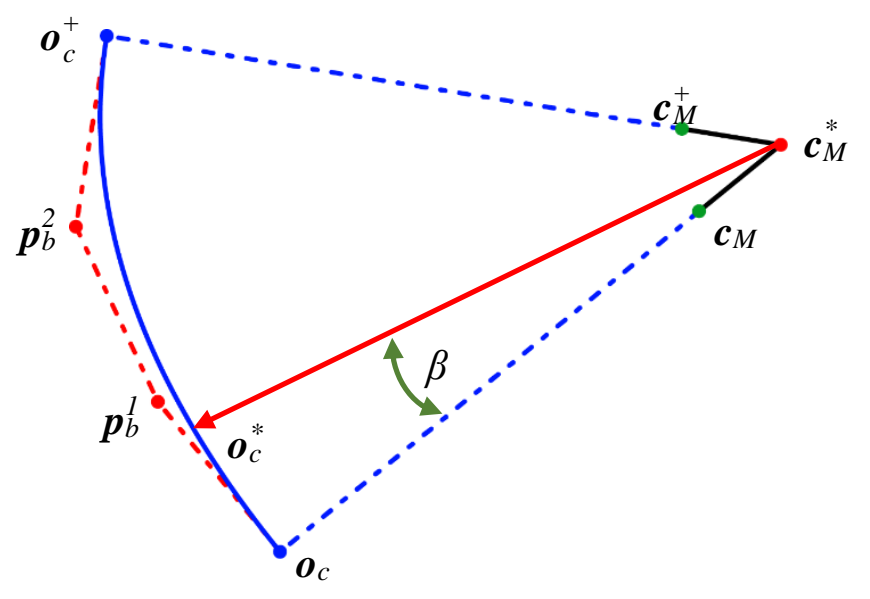

Figure 5.13: Path planning problem visualization.

A motion limit, via the angle $\beta$, is applied to limit the loss of $2 \mathrm{D}$ features experienced in the stereo camera pair due to the combined motion of the cameras and the deformation of the object. The stereo camera pair placement may alternatively be calculated directly along the curve as a function of the stereo-pair's motion parameters, namely, acceleration and velocity.

\subsection{Summary}

Herein, over Chapters 3-5, a methodology was developed for the shape recovery of a priori unknown deforming objects for a multi-camera active-vision system. The focus of the methodology was to maximize the shape recovery accuracy and completeness objectives through comprehensive model generation, deformation estimation and reconfiguration methods.

The model generation method ensures a complete estimate of the object's shape is produced through the fusion of triangulated surface patches and a visual hull. Triangulation of surface patches through calibrated cameras produces the highest possible shape recovery for localized shape recovery for a priori unknown objects. The visual hull model produces a complete bounding volume estimate of the target object. 
Through fusion, the visual hull model's accuracy is increased by removing excess volume and substitution of surface patches with triangulated patches. This approach produces a complete solid estimate of the target object with higher accuracy in triangulated regions and lower accuracy in visual hull regions. The model is represented with by a surface polygon model. The complete model estimate generated is crucial for the reconfiguration method to determine which viewpoints will improve the surface area visibility for stereo camera pairs. Further, the use of stereo-camera triangulation using key-based 2D image features enables object tracking and deformation estimation.

The deformation estimation method consists of an adaptive particle filtering algorithm that predicts the expected location of all tracked, triangulated 3D features of the target object. The predicted location of the $3 \mathrm{D}$ points is fused with the complete model to produce a singular deformation estimation. The algorithm adapts on-line to both the number of tracked points and the tracking variance to ensure the prediction precision tracks the 3D features' motion. The deformation estimate can then be used by the reconfiguration method to move cameras based on the expected deformation of the target object instead of the current objects' shape, potentially improving the shape recovered in the following demand instants.

The problem definition described the task of improving shape recovery as the maximization of surface visibility of a priori unknown objects. The purpose of multi-camera active-vision reconfiguration is to reposition stereo-camera pairs to improve the surface visibility of the $a$ priori unknown objects, thus improving the shape recovered. Camera repositioning can be approached as the selection of a combination of $c$ camera poses that maximize the visibility object. Thus, the reconfiguration method generalizes all stereo camera pairs available in the system to reduce the total number of computations necessary to find the near-optimal combination of stereo-camera positions that improve surface visibility. The camera repositioning workspace is discretized for all stereo camera pairs to ensure generality. A visibility estimation algorithm was developed to analyze the stereo-visibility of the expected object's deformation for all viewpoints in the discretized workspace simultaneously thanks to stereo-camera pair generalization. The cameras' paths were then planned through the workspace to ensure obstacle avoidance and maintain 2D feature tracking.

Ensemble, the methods developed result in a generic shape recovery system that can recover the shape of a priori unknown objects in obstacle-laden environments. The system developed can improve shape recovery over static multi-camera systems through reconfiguration and reduce processing overhead by reducing the total number of cameras necessary for recovery. 


\section{Chapter 6 Simulations}

Simulations were used to validate various individual components of the system along with the complete system within a controlled environment. A simulation environment allows for explicit of calibration camera parameters, optical parameters, and image distortions. Further, complete control of environmental lighting and target object control allows validation through ground-truth error analysis.

\subsection{Overview}

The simulations were conducted using MatLab ${ }^{\mathrm{TM}}$ and Blender ${ }^{\mathrm{TM}}$ [191] software, with Python ${ }^{\mathrm{TM}}$ scripting and the VLFeat library [192] for advanced computer-vision algorithms. MatLab was chosen for its robust programming interface that allows run-time debugging and code modification. The software can handle many potential errors encountered in vision processing due to its inherent data virtualization on the system. Specifically, run-time errors can be handled with exceptions unlike $\mathrm{C} / \mathrm{C}++$ programs that require specific run-time exception scripts. MatLab software runtime can be inherently slower than $\mathrm{C} / \mathrm{C}++$ implementations due to virtualization. Alternative implementations of the complete system through C/C++, OpenGL [193], OpenCL [194] and the Point Cloud Library (PCL) [195] will be discussed in the final chapter of the dissertation.

Blender ${ }^{\mathrm{TM}}$ is an open-source computer graphics program that allows model creation, texturization, animation and rendering. The program allows for scripted processing through two methods; Python scripting and command line scripting. Python scripts were interpreted by Blender and controlled all graphical user interface (GUI) elements. Command line scripting can be used within Windows ${ }^{\circledR}$ and $\operatorname{macOS} \AA$ to run Blender remotely, without the GUI, saving processing speed and reducing rendering time.

The VLFeat Library is an open-source programming library written in $\mathrm{C}$ with MatLab implementation. The library features a host of machine learning algorithms and computer-vision algorithms that were chosen for the system developed. These include SIFT feature detection and k-means clustering. Aside from feature detection, the library also includes comprehensive feature matching algorithms based on the source publications.

\subsection{Simulation Setup}

The complete simulation framework is presented in Figure 6.1. MatLab was used as the main integrated development environment (IDE). All methods and algorithms were coded as MatLab scripts. During runtime, MatLab calls Blender through the system command line with a pointer to the simulation file location, 
Python script, and script parameters. Blender opens the simulation file in the background and processes the Python script with input data. Five distinct Python script functions were written specifically for all simulations: exporting camera settings, exporting camera pose, exporting target object data, rendering, and setting camera parameters. Any external data input to Blender through a Python script function was handled as an external text file that was a priori generated by MatLab (i.e.: setting camera poses required MatLab to generate a text file with camera poses, and send a pointer to the file through the command line). Blender's output data included images in .jpeg format and text files for camera parameters and settings.

MatLab was programmed to wait while Blender completed all tasks. Once completed, MatLab would read the generated data from Blender as either images, or text file data, and continue with the system's process. The VLFeat library algorithms were implemented within the MatLab scripts as functions for locating SIFT features, matching SIFT features, and k-means clustering (VLFeat's implementation far outperformed MatLab's native k-means function in speed).

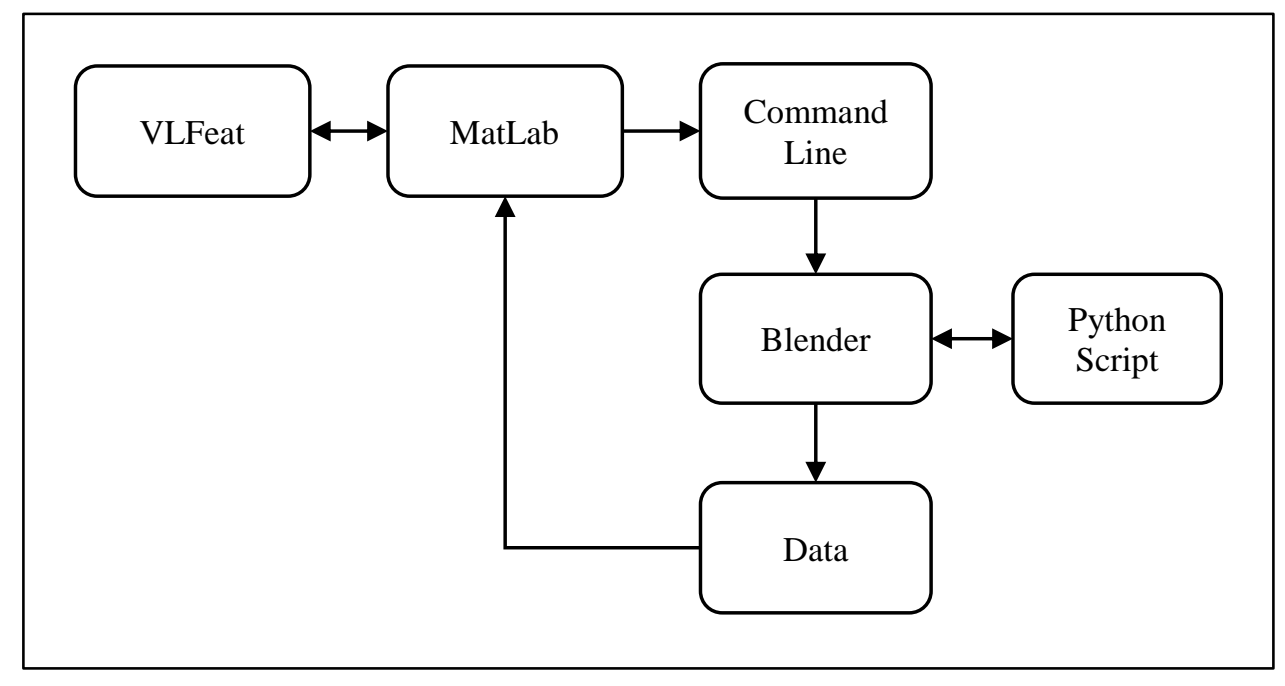

Figure 6.1: Simulation framework.

The remainder of this chapter will cover three distinct simulations for the purposes of validating the multi-camera active-vision system for shape recovery of a priori unknown deforming objects. The first simulation will focus on the generalized deformation estimation method, specifically, the adaptive particle filtering algorithm. The second simulation will focus on the validation of the model generation with deformation estimation methods combined. The third and final simulation will focus on the validation of the complete system including model generation, deformation estimation and reconfiguration. 


\subsection{Camera Calibration}

Camera calibration is a cornerstone of the proposed system. Fully calibrated cameras ensure the maximum shape recovery accuracy for stereo-camera pairs. Further, unlike partially calibrated or uncalibrated cameras, full camera calibration removes recovery ambiguities associated with the former. Some of these ambiguities were described in Section 3.4.1. Full camera calibration requires the intrinsic matrix, extrinsic matric and the radial and tangential distortions. The intrinsic matrix encodes the camera's focal length, center coordinate, and skew in pixel coordinates as a $[3 \times 3]$ matrix. The extrinsic matrix encodes the camera's position and orientation in world coordinates as a [3×4] matrix that includes the $[3 \times 3]$ rotation matrix, and relative translation vector $[3 \times 1]$ in homogeneous coordinates.

The Blender camera models implemented ideal perspective projection cameras with varying focal lengths. The depth of field was set to infinite range to simulate an ideally-calibrated camera with $a$ priori known focus ranges. The infinite DOF allowed the system to locate the stereo-camera pose along a placement vector that maximized the object's expected projection in both cameras. The radial and tangential distortions were set to zero. Image noise was added to each generated image in MatLab as random noise with uniform distribution.

\subsection{Background Segmentation}

The background separation process separates the target object and obstacles from the background and extracts the silhouette for each camera image at each sampling instant. The output of the segmentation is an image with labelled pixels that denote the target object, each unique obstacle, and the background. Many background segmentation methods exist in literature. For the purposes of the simulations, a simple script was developed similar to a morphological closing operation. To simplify the background segmentation problem, the simulation background was set to white, while the object's texture was set to a random color pattern without any white areas. The random color distribution of the object's texture ensured SIFT features will be located. The necessity of texture on the object's surface was explained in Section 3.4.2.

The background separation algorithm first converts the color image to gray-scale and, then, into a binary image by setting all non-white pixels to black through thresholding. A component-connection algorithm was implemented to identify all sets of 8-way connected pixels in the binary image. The component with the largest number of connected pixels is held constant, while all other pixels are set to zero. This eliminates the smallest connected groups of white pixels, typically, located within the object's silhouette. The resulting binary image is blurred with a Gaussian filter, and a binary threshold is once again applied to remove smaller groups of false-positive pixels. The new binary image mask is processed once more through a component-connection algorithm and, again, the pixels of the largest connected component 
are held constant, while the remaining pixels are set to zero, producing the final binary mask. The mask is used to determine the rectangular bounds about the object's silhouette. The silhouette pixel boundary is produced by removing all silhouette pixels with $[0,4]$ positive neighbors.

The background separation steps are presented in Figure 6.2: (a) initial input image with constant color background, randomly distributed surface textured object (this texture was used throughout the simulations), (b) color-thresholding of binary image, $(c)$ selection of largest connected pixel group of background color, $(d)$ Gaussian blurring, $(e)$ new thresholding of binary image, $(f)$ removal of internal silhouette pixels. Figure 6.2 intentionally consists of a more 'complex' object model to illustrate the effectiveness of the background separation utilized.

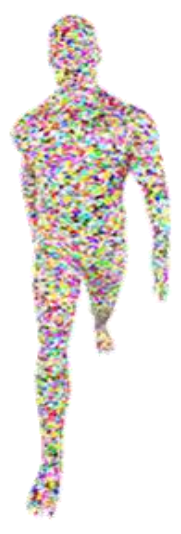

(a)

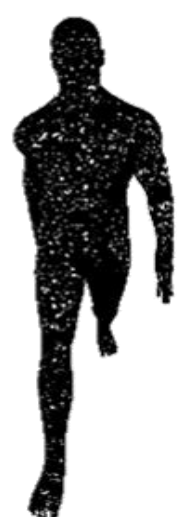

(b)

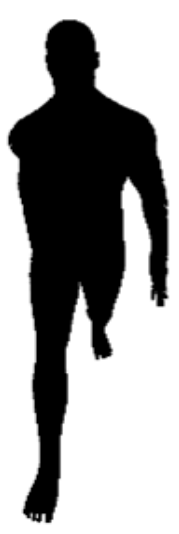

(c)

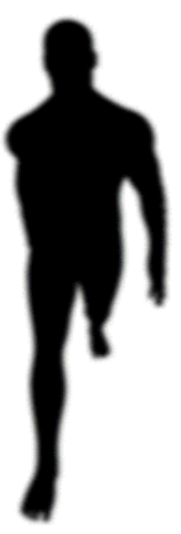

(d)

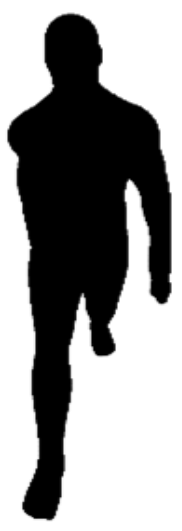

(e)

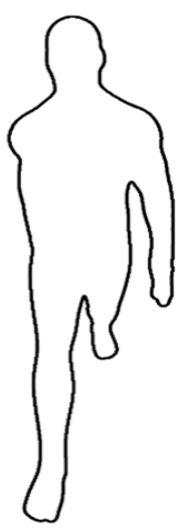

(f)

Figure 6.2: Background Separation Process: $(a)$ input image, $(b)$ color thresholding, $(c)$ selection of largest background connected group, $(d)$ Gaussian blurring, $(e)$ thresholding blurred image, and $(f)$ silhouette boundary identification.

\subsection{Deformation Estimation}

The deformation estimation methodology implements an adaptive particle filtering algorithm to simultaneously track many 3D triangulated features through a constant-acceleration motion model. The simulations for deformation estimation test the methodology's ability to accurately track varying surface deformations. These surface deformations represent the expected surface patch deformations for the multicamera active-vision system. Surface-based simulations were sufficient to test the accuracy of the deformation estimation methodology. The simulations also test the recall ability of the methodology to recover tracking when all features are lost. Three error metrics are presented for simulation validation, namely, triangulation errors, prediction errors, and prediction to triangulation errors. 


\subsubsection{Simulation Configuration}

Six example simulations are presented herein to demonstrate the proposed methodology. Each simulation utilized a fixed stereo-camera pair with a baseline of $200 \mathrm{~mm}$. All simulated camera models used a $32 \times 18 \mathrm{~mm}$ APC-style sensor, with an image resolution of $1920 \times 1080$ pixels, and $18 \mathrm{~mm}$ focal length. Uniformly-distributed color pixel noise was added to all images to simulate image noise. The addition of uniform noise acts as a worst-case scenario when compared to camera noise models presented in [174]. Furthermore, all triangulated data incorporated noise to simulate real-world errors in camera placement. The simulations used singular, textured, surface objects that deformed over a set of demand instants.

\subsubsection{Errors}

Three error metrics were used to analyze the performance of the methodology. The triangulation errors, $e_{t}$, were calculated as the Euclidean distance between a triangulated point and the nearest ground truth surface. The prediction errors, $e_{p}$, were calculated as the Euclidean distance between a predicted point and the nearest ground truth surface. The relative prediction errors, $e_{f}$, were calculated as the Euclidean distance between a predicted point locations and its actual triangulation location, for all tracked points. All three errors were normalized by the square root of the object's surface area. The normalization ensures the error metrics are invariant to the size of the target object, and relative pose of the cameras.

Triangulation errors were calculated as follows:

$$
e_{t}=\frac{1}{m \sqrt{S_{T}}} \sum_{j=1}^{m} z_{t}(j)
$$

where $z_{t}(j)$ is the shortest distance between the $j^{\text {th }}$ triangulated point in $\mathbf{X}_{\mathrm{TR}}$ to the surface of the true object model, $m$ is the total number of triangulated points at the given demand instant, and $S$ is the surface area of the true object model. This error metric quantifies the triangulation error that arises from SIFT feature matching inconsistencies, namely, the driving error for triangulation is based on the matching error since triangulation error is minimized by the ideal camera calibration.

Prediction errors were calculated as follows:

$$
e_{p}=\frac{1}{m \sqrt{S_{T}}} \sum_{j=1}^{m} z_{p}(j)
$$

where $z_{p}$ is the shortest distance between the $j^{\text {th }}$ predicted point in $\mathbf{X}^{+}$to the surface of the true object model, $m$ is the total number of predicted points. The prediction error quantifies how well the tracking predicts the expected deformation of the surface. 
The relative prediction errors were calculated as follows:

$$
e_{f}=\frac{1}{m \sqrt{S_{T}}} \sum_{j=1}^{m} z_{t}(j)-z_{p}(j) .
$$

The relative prediction errors represent the only surface-based error metric the system can use for feedback for a priori unknown objects. Specifically, without an object model for comparison, the only data the system available to the system, is the recovered shape. Therefore, the relative prediction errors represent the best system performance estimate. 


\subsubsection{Results}

The first simulation tested the method's robustness to varying number of available features by moving the surface object toward to the cameras. A four-frame movie-strip of the object is presented in Figure 6.3. As expected, the error decreased as the object approached the cameras and the number of tracked and triangulated points increased, Figure 6.4. The errors remained under $1.2 \%$ for all three metrics.

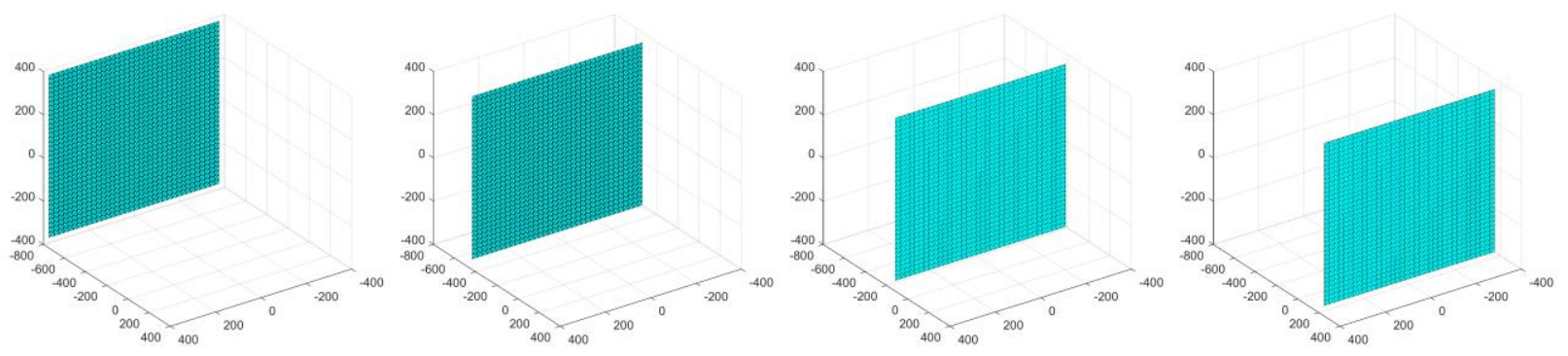

Figure 6.3: Movie-strip for Simulation 1.
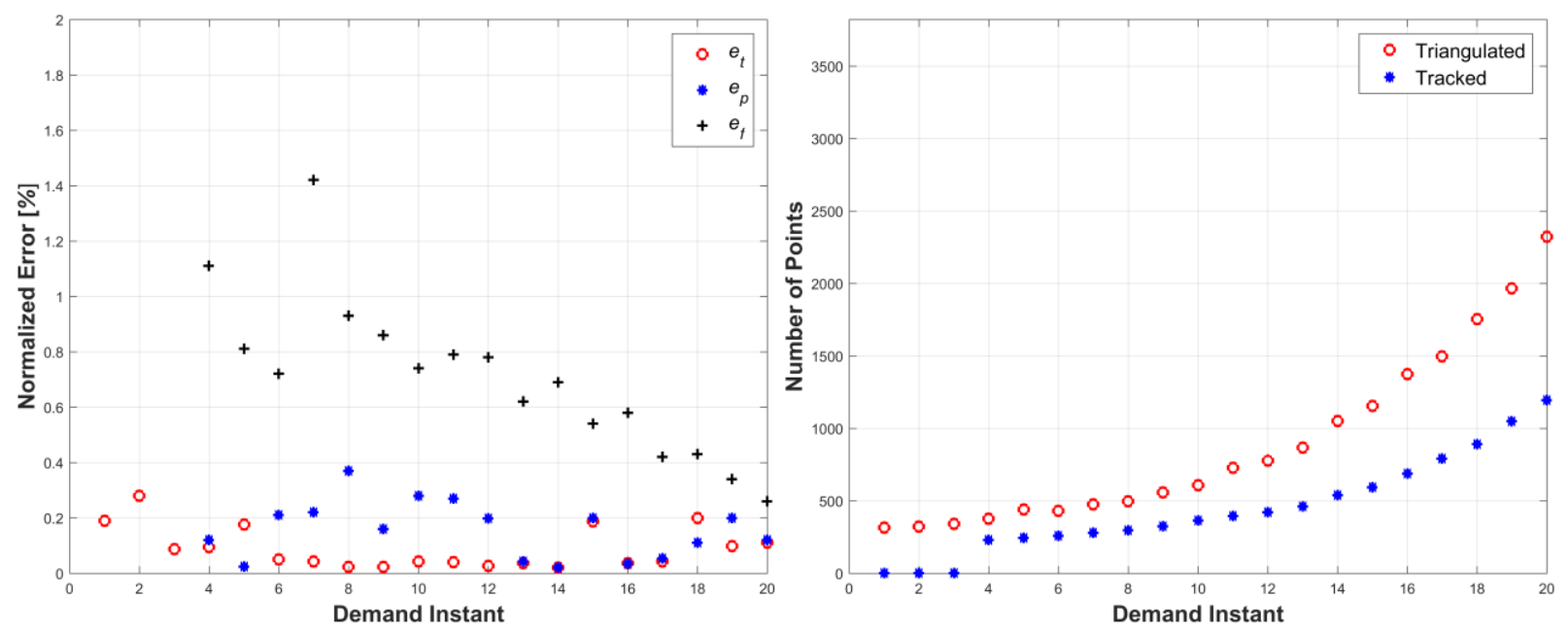

Figure 6.4: Left: triangulation, prediction, and relative prediction error metrics for each demand instant. Right: total number of tracked and triangulated points for each demand instant. 
The second simulation consisted of an object surface stretching toward the cameras. A four-frame movie-strip of the object is presented in Figure 6.5. This simulation tested the proposed methodology's ability to handle an arbitrary, simple, deformation. The errors remained under $1.2 \%$ for all three error metrics, and the total number of tracked and triangulated points remained stable, Figure 6.6.
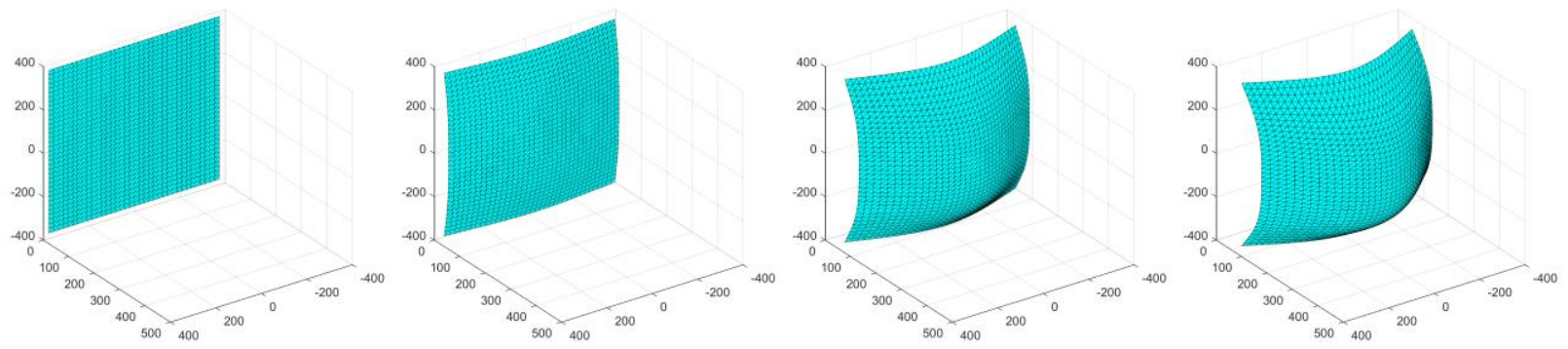

Figure 6.5: Movie-strip of Simulation 2.
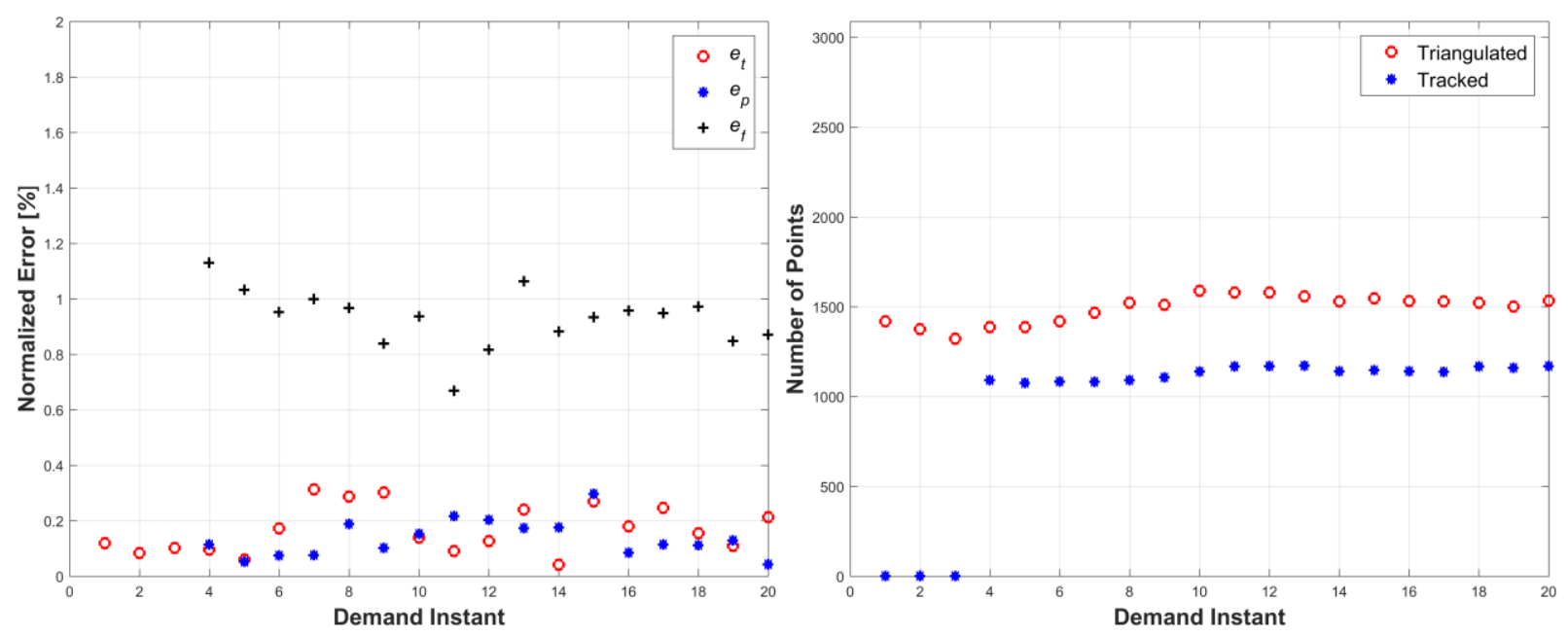

Figure 6.6: Left: triangulation, prediction, and relative prediction error metrics for each demand instant. Right: total number of tracked and triangulated points for each demand instant. 
The third simulation consisted of an object surface undergoing a wave-like stretching. A four-frame movie-strip of the object is presented in Figure 6.7. This simulation tested the methodology's ability to handle a more complex form of surface deformation. The errors remained under $1 \%$ for all three error metrics, and the total number of tracked and triangulated points remained stable with a slight increase, Figure 6.8.
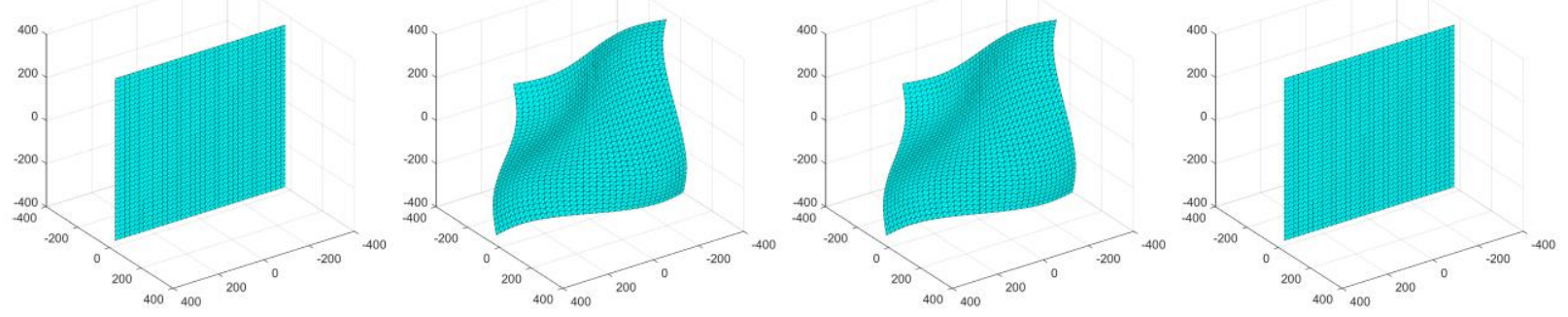

Figure 6.7: Movie-strip of Simulation 3.
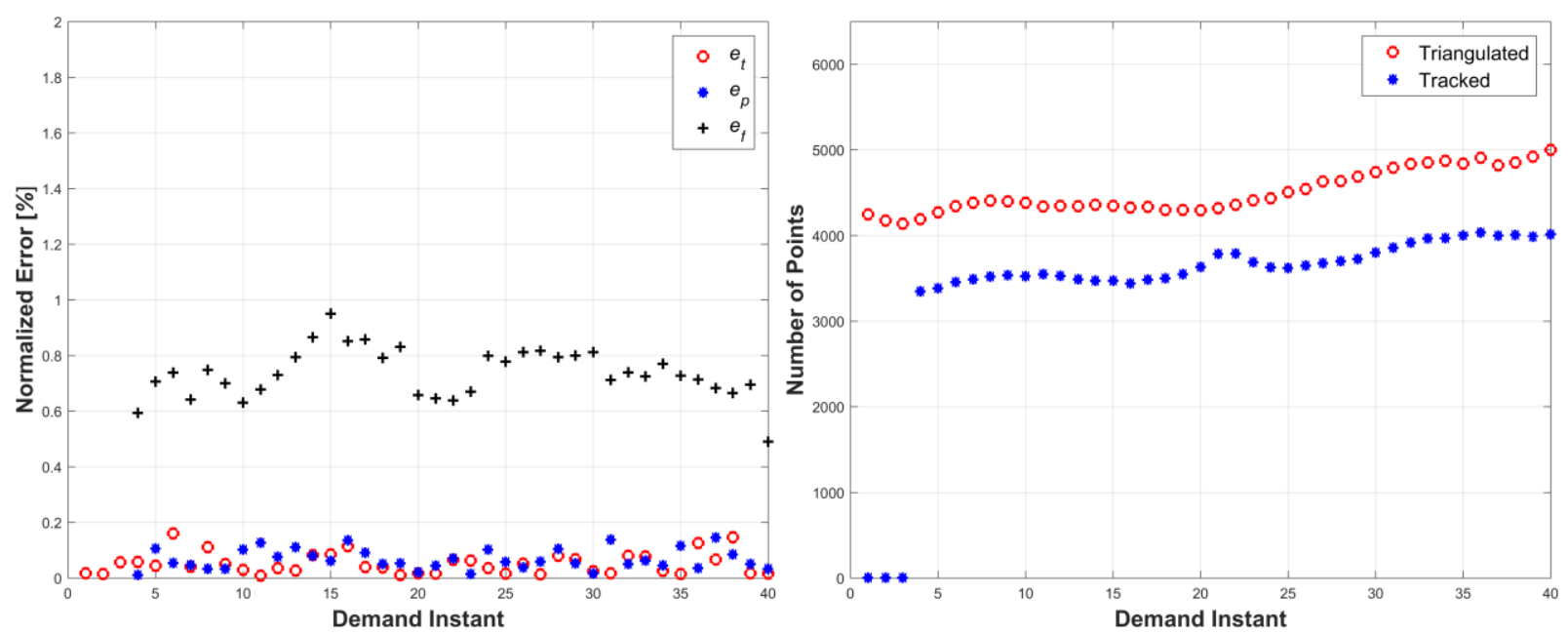

Figure 6.8: Left: triangulation, prediction, and relative prediction error metrics for each demand instant. Right: total number of tracked and triangulated points for each demand instant 
The fourth simulation consisted of an object surface undergoing a wave-like stretching deformation with a linear translation. A four-frame movie-strip of the object is presented in Figure 6.9. This simulation tested the methodology's ability to handle a more complex form of surface deformation with global motion. The errors remained under $1.3 \%$ for all three error metrics, and the total number of tracked and triangulated points remained stable with a slight increase, Figure 6.10. The slight increase in error from the third simulation is attributed to the compounded motion experienced by every tracked and triangulated point. Namely, in the previous simulation, the tracked points only moved in the wave motion, while in this example, they also moved perpendicularly to the wave motion, thus, increasing the relative prediction errors.
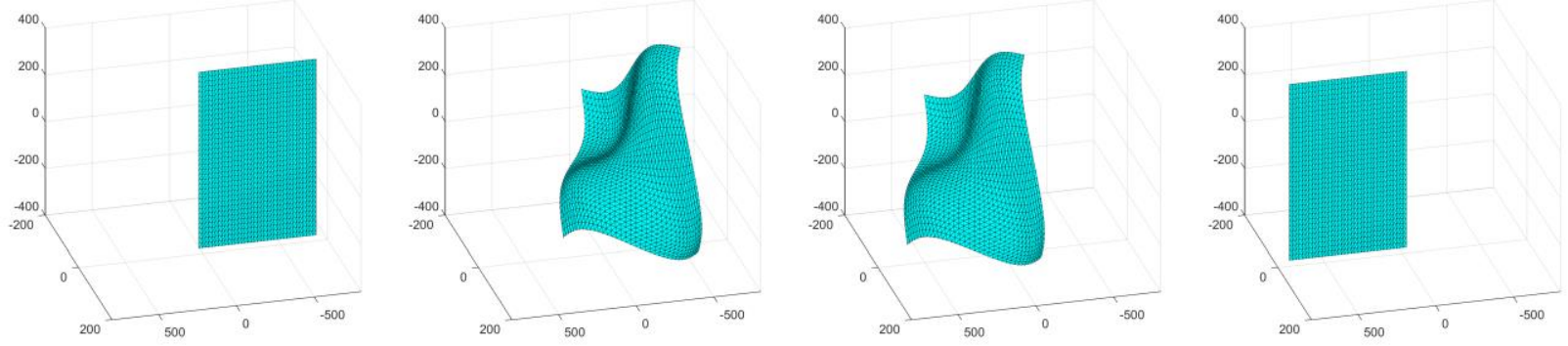

Figure 6.9: Movie-strip of Simulation 4.
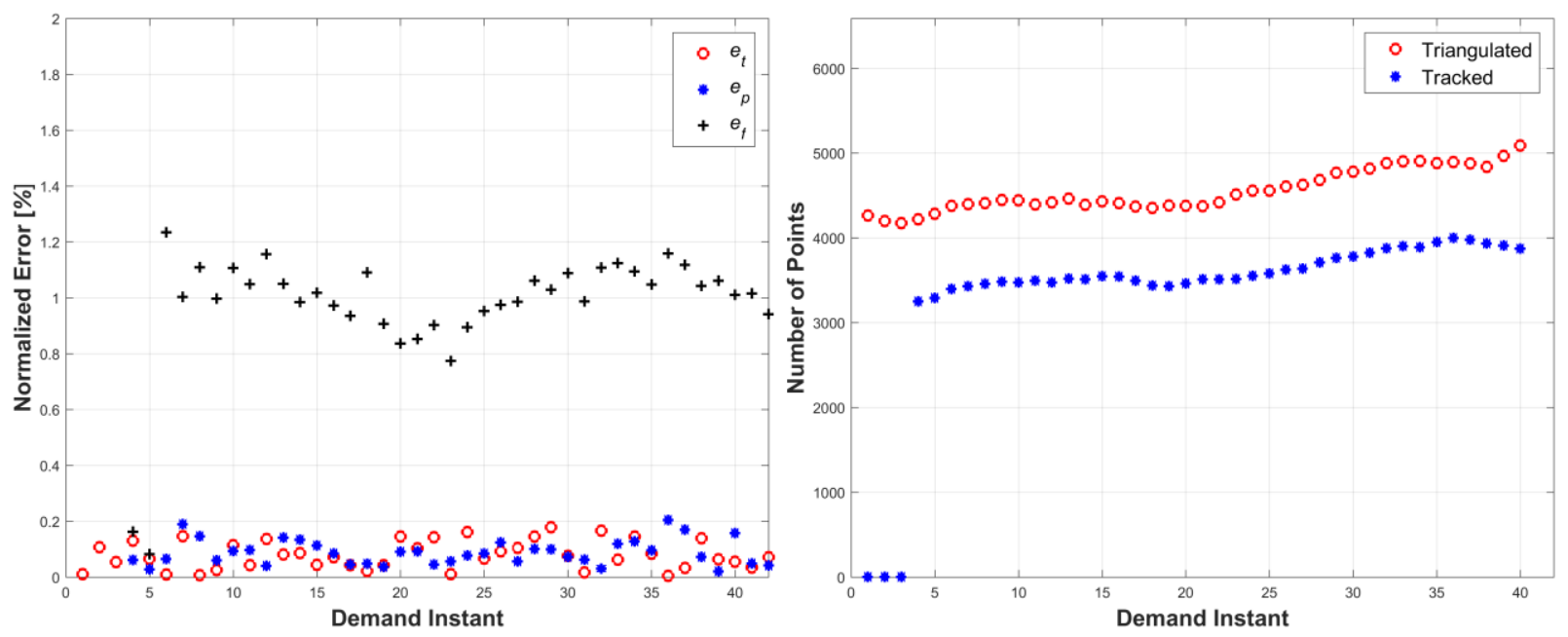

Figure 6.10: Left: triangulation, prediction, and relative prediction error metrics for each demand instant. Right: total number of tracked and triangulated points for each demand instant 
The fifth simulation consisted of an object surface undergoing a wave-like stretching with linear translations in two directions. A four-frame movie-strip of the object is presented in Figure 6.11. This simulation tested the methodology's ability to handle a more complex form of surface deformation with increased global motion. The errors remained under $1.4 \%$ for all three error metrics, and the total number of tracked and triangulated points gradually increased as the object moved closer to the cameras, Figure 6.12. The cyclic error pattern for the relative prediction errors, $e_{f}$, is attributed to the acceleration profile of the motion where the lowest error values correspond to zero acceleration of the object.
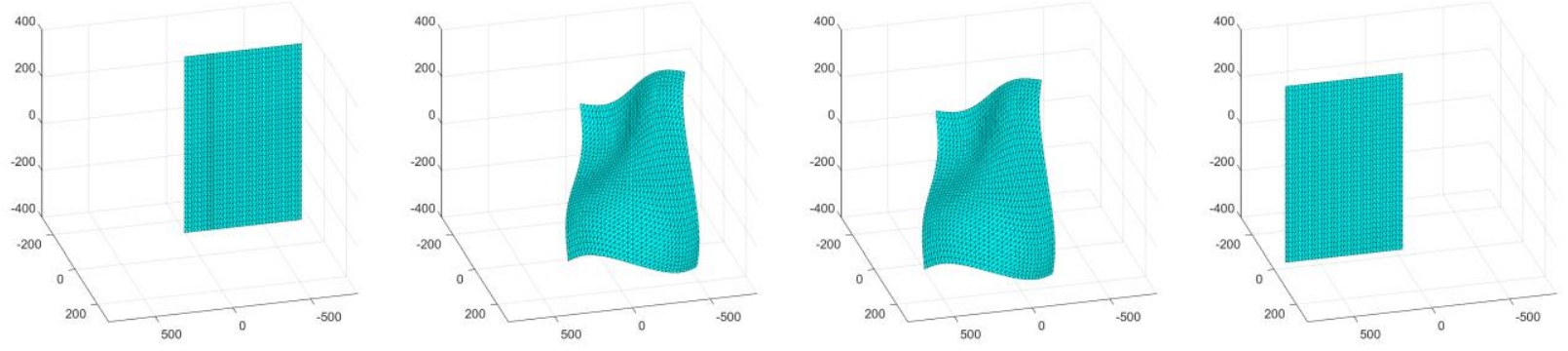

Figure 6.11: Movie-strip of Simulation 5.
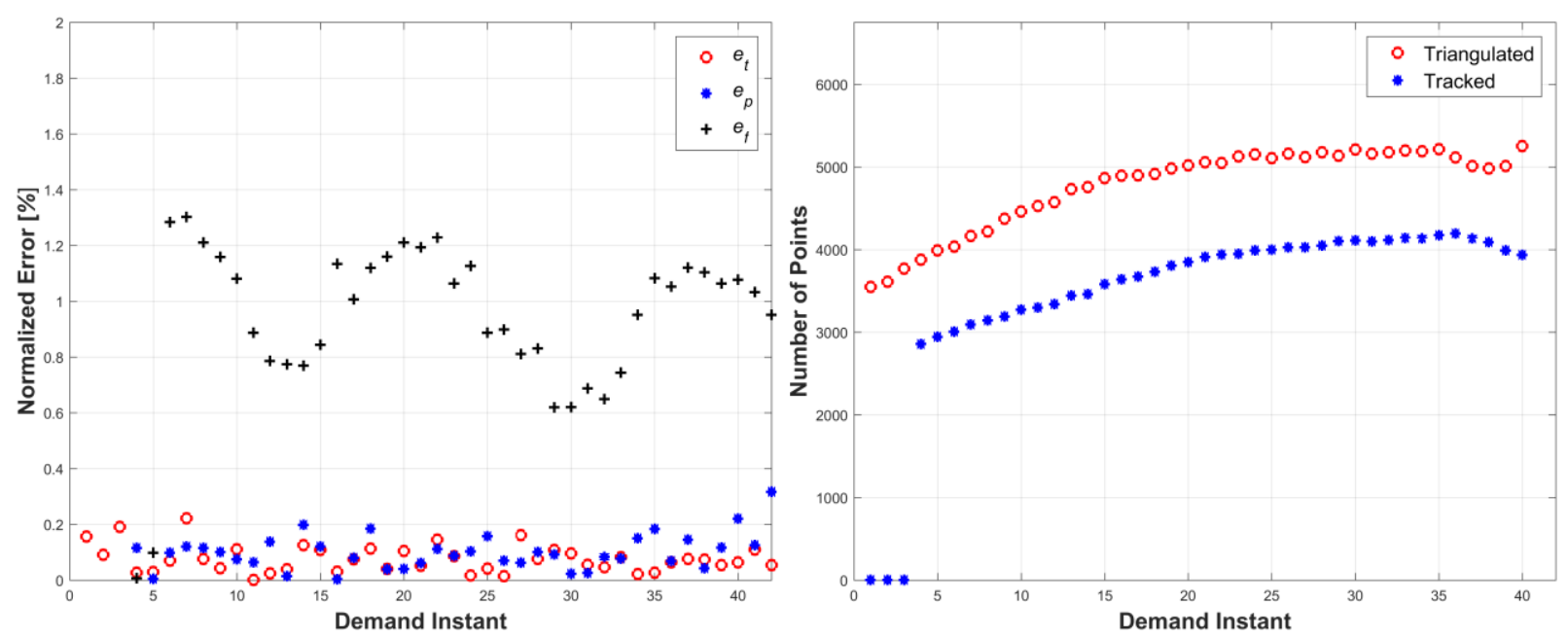

Figure 6.12: Left: triangulation, prediction, and relative prediction error metrics for each demand instant. Right: total number of tracked and triangulated points for each demand instant. 
The sixth simulation consisted of a surface object undergoing a wave-like stretching with a linear translation and a rotation by $180^{\circ}$. A four-frame movie-strip of the object is presented in Figure 6.13. This simulation tested the methodology's ability to handle complex surface deformation with complete loss of visibility. The errors remained under $2 \%$ for all three error metrics with an increasing trend as the object rotated to a parallel orientation with respect to the cameras, at which point none of the triangulated points could be tracked (due to loss of tracking data), Figure 6.14. The number of tracked and triangulated points reflected the error behaviour. Namely, as the object became parallel to the optical axes, the number of triangulated points decreased to almost zero, while all the tracked points were lost.
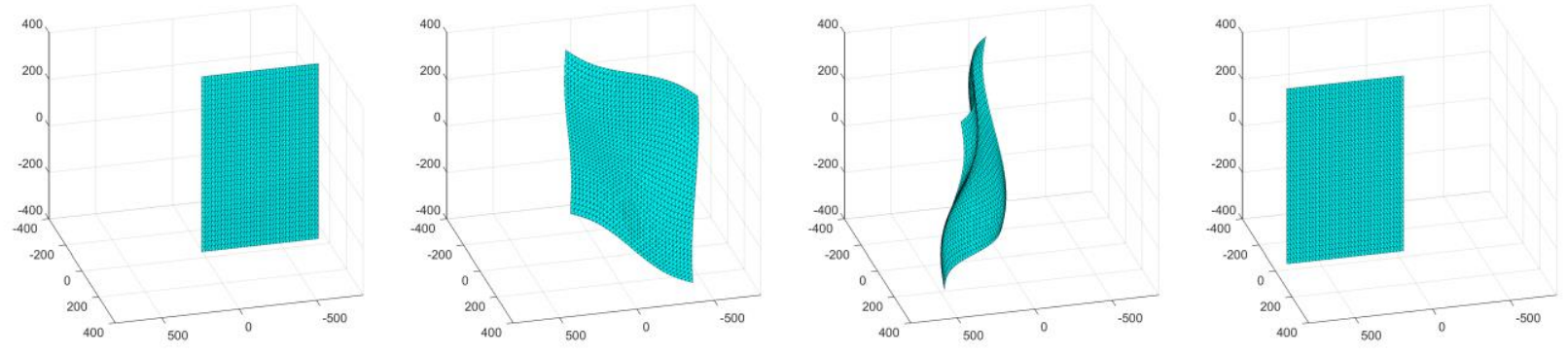

Figure 6.13: Movie-strip of Simulation 6.
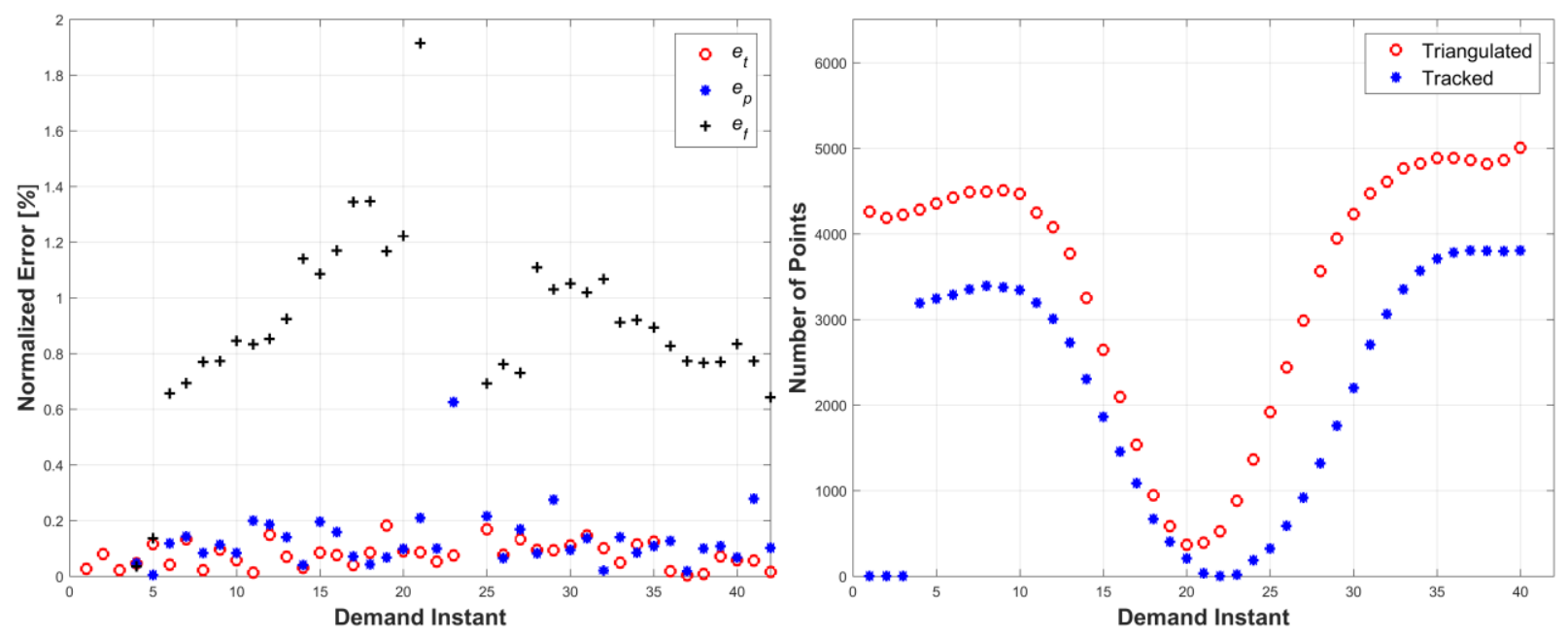

Figure 6.14: Left: triangulation, prediction, and relative prediction error metrics for each demand instant. Right: total number of tracked and triangulated points for each demand instant. 


\subsection{Model Generation and Deformation Estimation}

The model generation methodology was tested in simulation directly with deformation estimation. The purpose of the simulations was to test the combined methods' ability to predict the complete deformation of an a priori unknown target object. The results of these simulations were compared to another fusion method proposed in literature. Further, the combined method was tested with a priori known objects and the results compared to the a priori unknown simulations. Both comparisons and supplementary simulation data is presented in Appendix C. The simulations configurations were similar to the deformation estimation simulations. A new set of normalized errors were developed for the combined methodology based on the surface area accuracy and volumetric accuracy of the recovered models and their deformation predictions.

\subsubsection{Simulation Configuration}

All simulations used six cameras placed around the object and configured into three stereo-camera pairs. Each camera was modeled with a $32 \times 18 \mathrm{~mm}$ APC-style sensor, $1920 \times 1080$ pixel resolution, and $18 \mathrm{~mm}$ focal length. All simulations comprised 50 demand instants, with objects deforming through each. Each image was rendered with added noise in all color channels, and all triangulated points included added triangulation noise to model real-world errors in camera calibration parameters. The simulations used single solid, textured, homogeneous object that deformed over a set of demand instants.

These simulations used 'basic' convex shapes that deformed into complex concave shapes over a set of sampling instants to illustrate the robustness of the method to deforming geometries. The purpose of using basic shapes was to illustrate the general robustness of the model generation and deformation estimation methodologies to objects with varying surface areas and volumes, without complex mesh generation algorithms necessary for highly-detailed objects. The use of these models demonstrates the ability to accurately model and predict the deformation of the a priori unknown object.

\subsubsection{Errors}

Four error metrics were used to analyze the performance of the proposed method. The error metrics were calculated for the normalized surface area error, the normalized volumetric error, the normalized triangulation error, and the normalized visual hull error.

The normalized surface area errors are calculated as follows:

$$
e_{s}=\frac{\left|S^{+}(t)-S_{T}(t+1)\right|}{S_{T}(t+1)},
$$


where $S^{+}(t)$ is the surface area of the predicted deformation for the next demand instant, and $S(t+1)$ is the surface area of the true model at the next demand instant. This error metric is unknown to the system and represents the accuracy with which the predicted deformation model's surface area compares to the true object's deformation at the next demand instant.

The normalized volumetric errors are calculated as follows:

$$
e_{v}=\frac{\left|V^{+}(t)-V_{T}(t+1)\right|}{V_{T}(t+1)},
$$

where $V^{+}(t)$ is the volume of the predicted deformation at time for the next demand instant, and $V(t+1)$ is the volume of the true model at the next demand instant. This error metric is also unknown to the system due to its dependence on the true object model.

The normalized triangulation errors are calculated as follows:

$$
e_{T R}=\frac{1}{n \sqrt{S_{T}(t+1)}} \sum_{i=1}^{n} z_{T R}(i),
$$

where $z_{T R}(i)$ is the shortest distance between each predicted triangulated point in $\mathbf{X}_{T R}^{+}$and the true object at the next demand instant model normalized by the square root of the total surface area at the next demand instant. This error is similar to the error in Equation (6.2). The normalizing constant of the square root of the total surface area was chosen as it can be applied to both triangulation, and visual hull errors and provides a clear representation of the scale of the errors relative to the scale of the object.

The normalized visual hull errors are calculated as follows:

$$
e_{V H}=\frac{1}{m \sqrt{S_{T}(t+1)}} \sum_{j=1}^{m} z_{V H}(\mathrm{j}),
$$

where $z_{V H}(i)$ is the shortest distance between each visual hull points in $\mathbf{X}_{V H}^{+}$and the true object model at the next demand instant normalized by the square root of the total surface area at the next demand instant.

\subsubsection{Results}

The first simulation, 'Sph', comprised a sphere deformed by a bounding lattice, with rotation about the global $y$ - and $z$-axis of $90^{\circ}$ and $45^{\circ}$, respectively. The results of the simulation are presented in Figure 6.15, wherein the true 3D model of the object was compared to the recovered model. All view points were taken from MatLab. A second viewpoint in MatLab of the simulation is provided in Appendix B to illustrate the accuracy of the recovered shape. The normalized errors for the simulation are presented in Figure 6.16. The 
total normalized errors for surface area and volume were under $6 \%$ with an average of $1.8 \%$ error for surface area and 3.0\% for volume. Similarly, the triangulation and visual hull errors were below $1.0 \%$, with an average of $0.3 \%$ error for visual hull points and $0.02 \%$ for triangulated points.

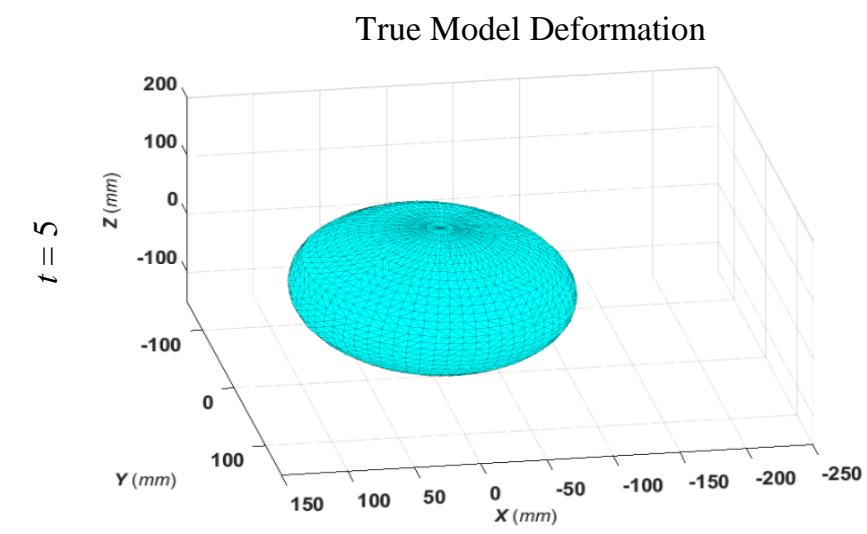

Fused Model, Deformation Prediction

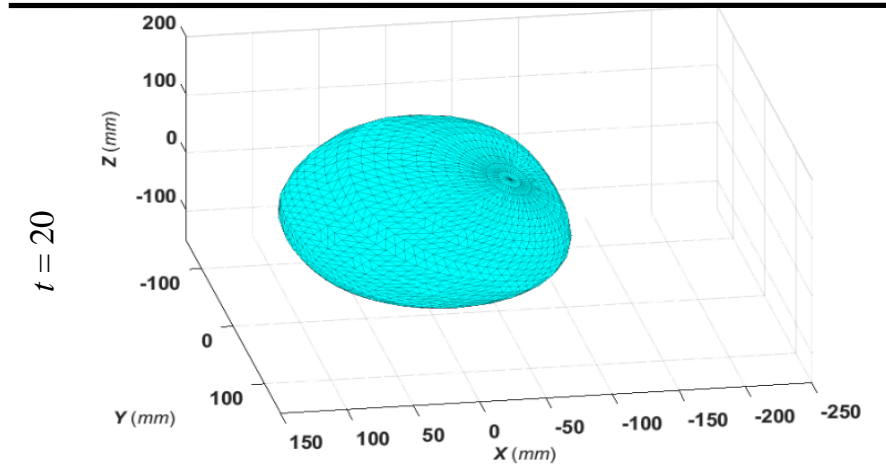

\begin{tabular}{llllllllll}
150 & 100 & 50 & $\begin{array}{l}0 \\
X(\mathrm{~mm})\end{array}$ & -50 & -100 & -150 & -200 & -250 \\
\hline
\end{tabular}
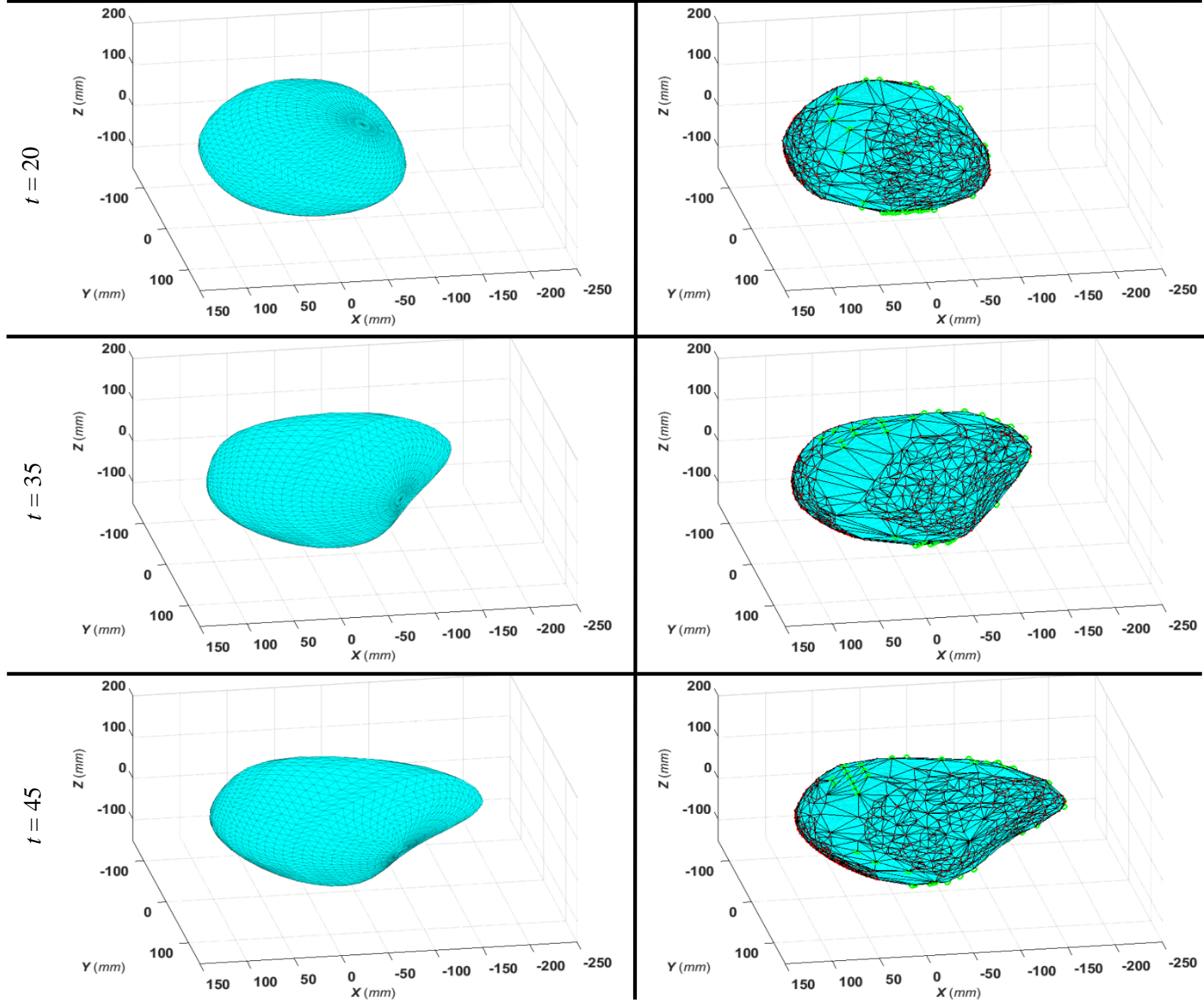

Figure 6.15: Results for Simulation 'Sph'. 


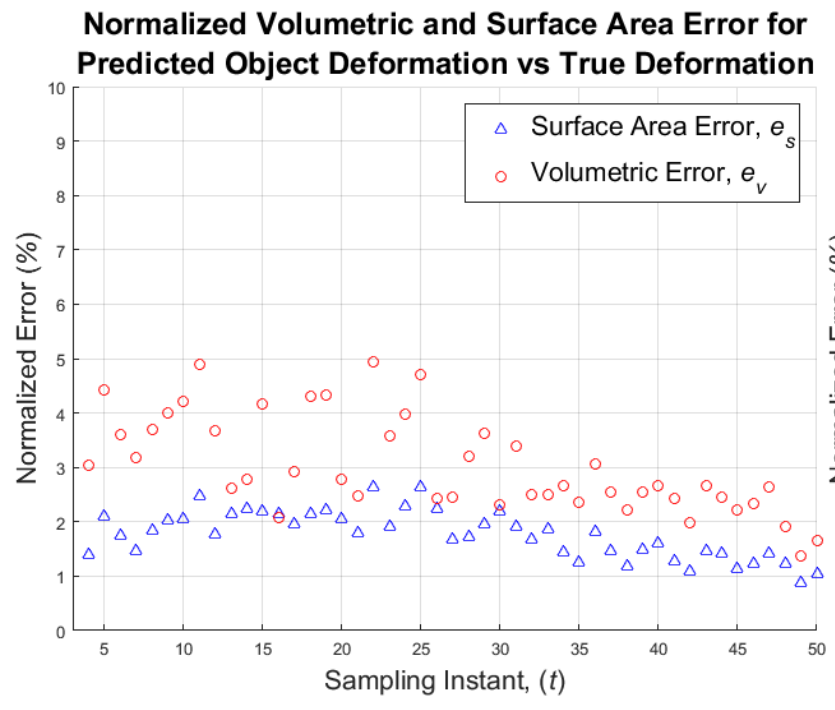

(a)

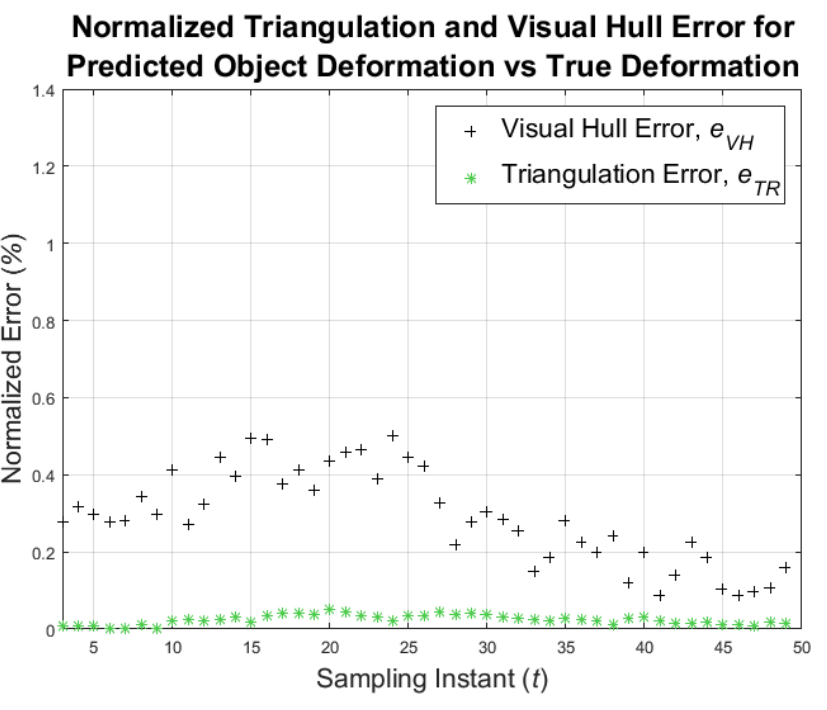

(b)

Figure 6.16: Normalized Errors for (a) Surface and Volume, and (b) Triangulation and Visual Hull Data.

The second simulation, 'Prl' configuration 'II', comprised a smooth-edge rectangular prism deformed by a curve and rotated $45^{\circ}$ about the global $z$-axis. The proposed method was capable of nearcomplete model estimation at each sampling instant through the fusion technique. The results of the simulation are presented in Figure 6.17, as a comparison between the true object's model and the fused model of the predicted deformation for Sampling Instants 5, 20, 35, and 45, respectively. A second viewpoint of the object is provided in Appendix B, illustrating the complexity of deformation. The accuracy of the predicted deformation is presented in Figure 6.18. The total normalized errors for surface area and volume were under $7 \%$ with an average of $1.6 \%$ error for surface area and $1.4 \%$ for volume. Similarly, the triangulation and visual hull errors were below $1.5 \%$, with an average of $0.3 \%$ error for visual hull points and $0.02 \%$ for triangulated points.

Alternative camera configurations and comparisons of the model generation and deformation estimation methodologies are presented in Appendix C. The data in Appendix C includes a third simulation, 'Pr2', and performance evaluation for multiple camera configurations. The simulation results are also compared with a model generation methodology from literature, [59]. Finally, the simulations were also compared to results acquired using the a priori known object model. The supplementary data shows the improvement of the developed system in comparison to current methods in literature and comments on the importance of camera viewpoint selection. The importance of viewpoint selection results is the basis of this dissertation. 


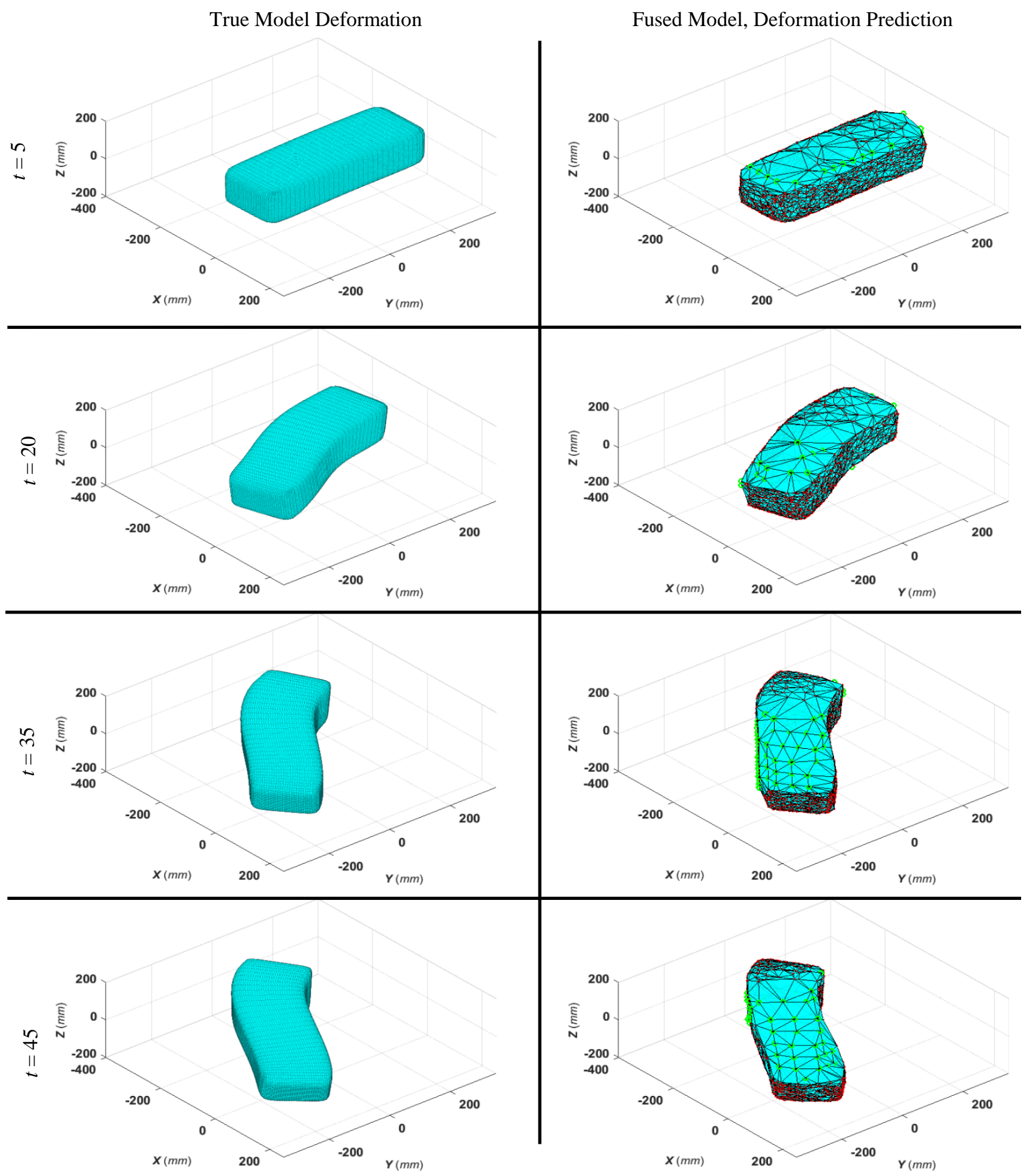

Figure 6.17: Results for Simulation 'Prl', Configuration 'II'. 


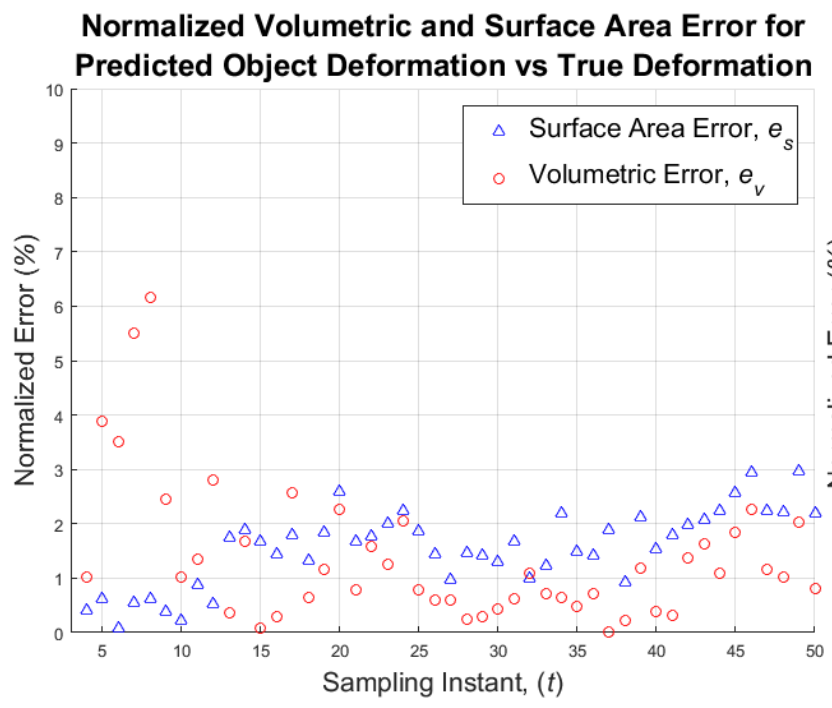

(a)

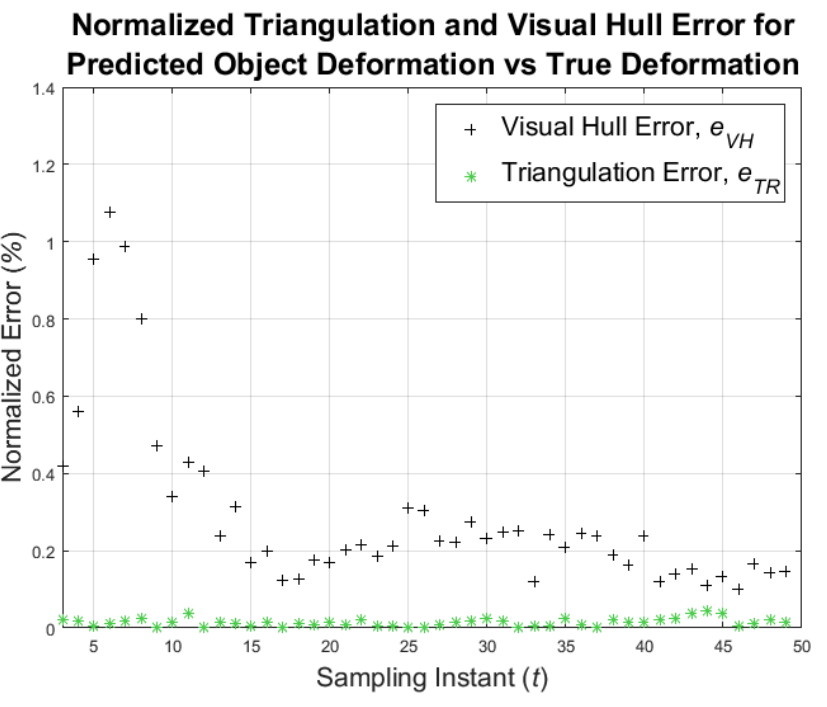

(b)

Figure 6.18: Normalized Errors for (a) Surface and Volume, and (b) Triangulation and Visual Hull Data.

\subsection{Complete Methodology}

The complete methodology was tested using multiple simulation to validate the benefit of reconfiguration in comparison to static cameras and ideal cameras. The simulations for model generation and deformation estimation mentioned the importance of viewpoint selection when recovering the shape of an a priori unknown target object. Herein, several simulations were run to test the complete multi-camera active-vision reconfiguration system for the shape recovery of a priori unknown deforming objects. The error metrics developed for simulation validation follow the progression of the previously developed metric in Equations (6.1) - (6.7), and integrate the principles for shape recovery reconfiguration from Equations (2.5), (5.8), and (5.9).

\subsubsection{Simulation Configuration}

A total of 36 simulation runs were conducted in 12 sets of three. All simulations used six cameras placed around the object into three stereo-pairs. Each camera was modelled with a $32 \times 18 \mathrm{~mm}$ APC-style sensor, $1920 \times 1080$-pixel resolution, and $70 \mathrm{~mm}$ focal length. Image noise was added in MatLab. The long focal length was used to reduce foreshortening effect. The target object was modelled as a smooth-edge rectangular prism embedded with a kinematic deformation armature with 11 bones. The deformation consisted of curling the bones along the $x$ - $y$ plane to yield a strong curvature in the object. The obstacle was modelled as a cylinder in MatLab environment to simplify image segmentation. 
Each simulation set comprised three different camera placement conditions: (i) static cameras, (ii) reconfigurable cameras, and (iii) ideal cameras. Each simulation used a different initial stereo-pair placement relative to the object, namely, different rotation of the camera pairs, and different vertical offset from the object. The static camera simulations and ideal camera simulations are presented for comparison purposes to illustrate how the system developed strives to approach the ideal placement results. The ideal simulations represent the theoretical maximum achievable surface area visibility.

The static cameras were placed on a single plane oriented towards the center of the workspace with a stereo disparity of $200 \mathrm{~mm}$, with a radial distance of $3000 \mathrm{~mm}$ about the center. Each stereo-pair was separated by $120^{\circ}$ from the other two, an example camera configuration is presented in Figure 6.19. The static camera placement was used as the initial camera placement for both reconfiguration and ideal simulations. For a fair comparison, camera placements were limited to the $x-y$ plane, and centered about the target object. Namely, the reconfiguration and ideal simulations positioned the cameras radially about the target object. The reconfiguration simulations used the proposed method described in this paper, while the ideal simulations reconfigured the cameras based on an a priori known model deformation using the same visibility function and reconfiguration strategy.

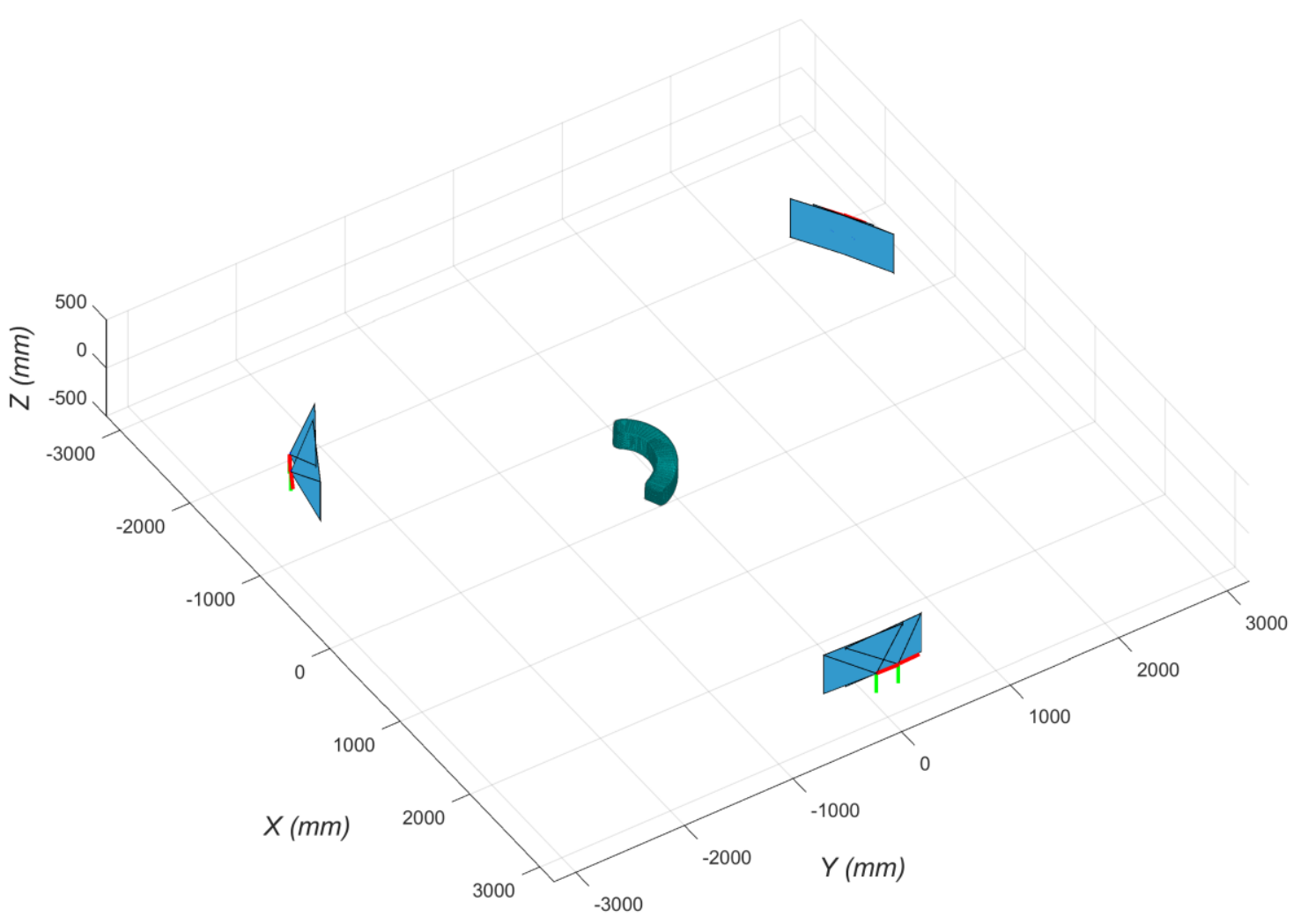

Figure 6.19: Example camera configuration about the target object. 


\subsubsection{Errors}

The methodology and simulations leading up to this section describe that maximizing the stereo visibility of the a priori unknown target object will result in the maximization of the shape recovery objectives, namely, accuracy and completeness. The accuracy of the recovered shape is handled through stereo triangulation from fully calibrated cameras coupled with an adaptive particle filtering algorithm. The completeness of the model is handled through a fusion methodology for stereo-triangulated surface patches and a visual hull. Thus, by delegating the accuracy and completeness tasks to the model generation and deformation estimation methods, the complete system can be evaluated by a single stereo-visibility metric based on Equations (2.5), (5.8), and (5.9).

The evaluation metric chosen for complete system performance, $\eta_{s y s}$, was the ratio of the estimated surface area visible from stereo-cameras to the true surface area of the object. This metric, like previous metrics, is unknown to system and is only used herein for performance evaluation. The metric was calculated as follows:

$$
\eta_{s y s}=\frac{\beta_{v i s}}{S_{T}}
$$

where $\beta_{v i s}$ is the estimated surface area visible from stereo-cameras. The estimated was calculated through a ray-triangle intersection algorithm. Each camera pair produced a point cloud representing a surface patch of the object. The triangulated surface patch was, then, subdivided, and its vertices and corresponding stereo-pair centers $\left(\boldsymbol{c}_{M}\right)$ were stored.

All the vertices and centers were cast as rays in the workspace, and their intersections with the true object model were recorded. If the intersection between the ray and model polygon was in the proximity of the ray's vertex, the polygon was labelled as visible. The surface area visibility metric was, then, calculated as the sum of the areas of the individual visible polygons divided by the total surface area of the model, where the sum of all visible polygons produced $\beta_{v i s}$.

\subsubsection{Results}

The results of each of the 12 simulation sets are presented in Figure 6.20 - Figure 6.23. Each individual plot shows the total surface area captured through stereo-triangulation for static, reconfiguration and ideal camera placement for each demand instant. Mean value bars were added to illustrate the average surface area recovered for each simulation. Sample overhead sequences of a simulation are provided in the Appendix D. 
During the simulations, an obstacle moved to occlude one pair of static cameras at the third demand instant, and moved out of the field of view at the $16^{\text {th }}$ demand instant. This occlusion resulted in approximately $15-20 \%$ loss of visibility relative to the ideal case. In Simulations $\{1,2,5,6,9,10\}$, the object's deformation resulted in self-occlusion that could not be resolved by the static cameras. The loss due to self-occlusion was approximately $5 \%$. In Simulations $\{3,4,7,8,11,12\}$, the static cameras were positioned such that the self-occlusion could be resolved, thus, the surface area recovered past the $16^{\text {th }}$ demand instant approached that of the ideal cameras.

The multi-camera active-vision system developed herein approached the performance of the ideal camera placements for all simulations. The deviation of the ideal configurations is attributed to the surface area evaluation metric calculation. Since the evaluation metric relied on the ray-triangle intersection of triangulated points, some variance was induced due to the total number of triangulated features at any given demand instant. The simulation results clearly indicate that the proposed reconfiguration method is capable of outperforming static camera configurations while trending toward the ideal maximum surface area visibility. Furthermore, the reconfiguration method could overcome the object's self-occlusion in the first demand instant as seen in Simulations $\{2,3,7,10,11\}$, where the ideal cameras could improve the surface area recovered in the $2^{\text {nd }}$ and $3^{\text {rd }}$ demand instants - the reconfiguration method required more time to approach the ideal results. Simulation 6 illustrates a similar case, but the reconfiguration took longer to approach the ideal results. In Simulations $\{1,8,12\}$, the reconfiguration method yielded results that approached the ideal results, but the cameras were not positioned to handle the object's self-occlusion near the end of the sequence, resulting in some loss of performance.

Overall, the reconfiguration method attempted to firstly overcome the obstacle occlusion, and secondly, overcome the object's self-occlusion. Overcoming self-occlusion was only possible if the cameras could achieve a feasible position given the motion constraints imposed. In contrast, unconstrained cameras in the ideal simulations were always able to overcome both the obstacle occlusion and selfocclusion as noted by the lack of performance degradation in the last four frames. It is noted that the performance benefit of the reconfiguration is greatly improved in the presence of obstacles. 

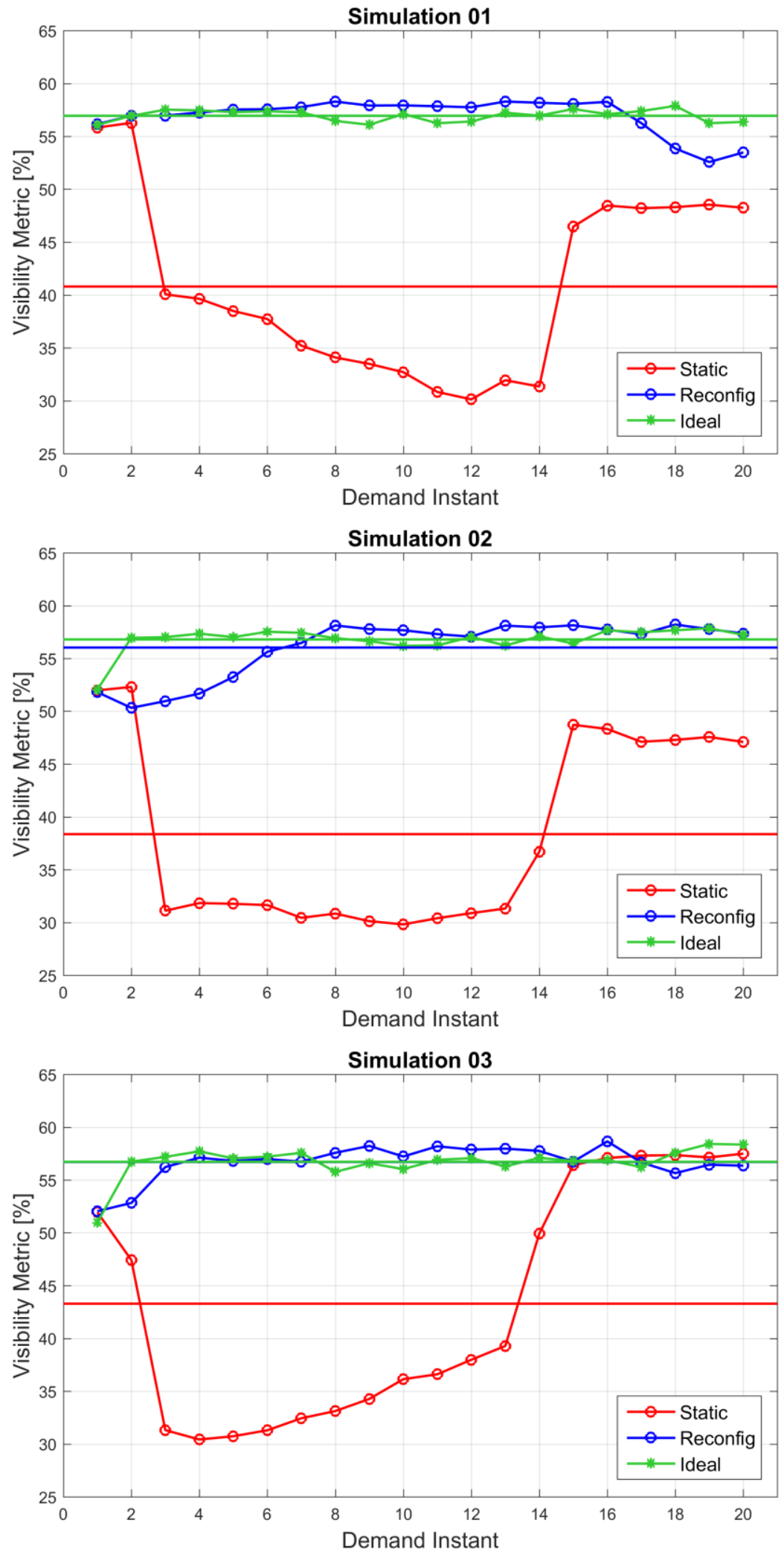

Figure 6.20: Surface area results for Simulations 1-3. 

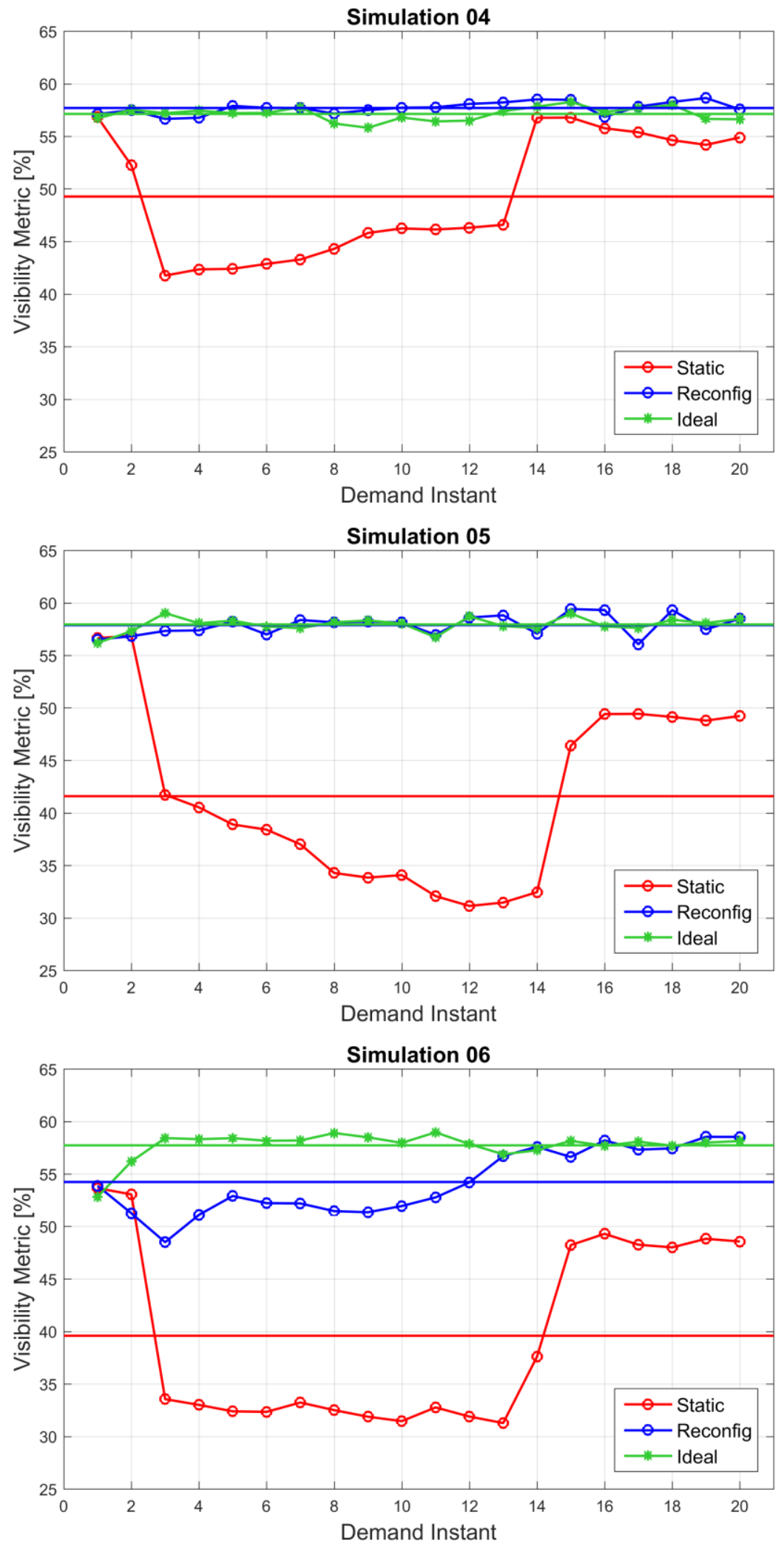

Figure 6.21: Surface area results for Simulations 4-6 

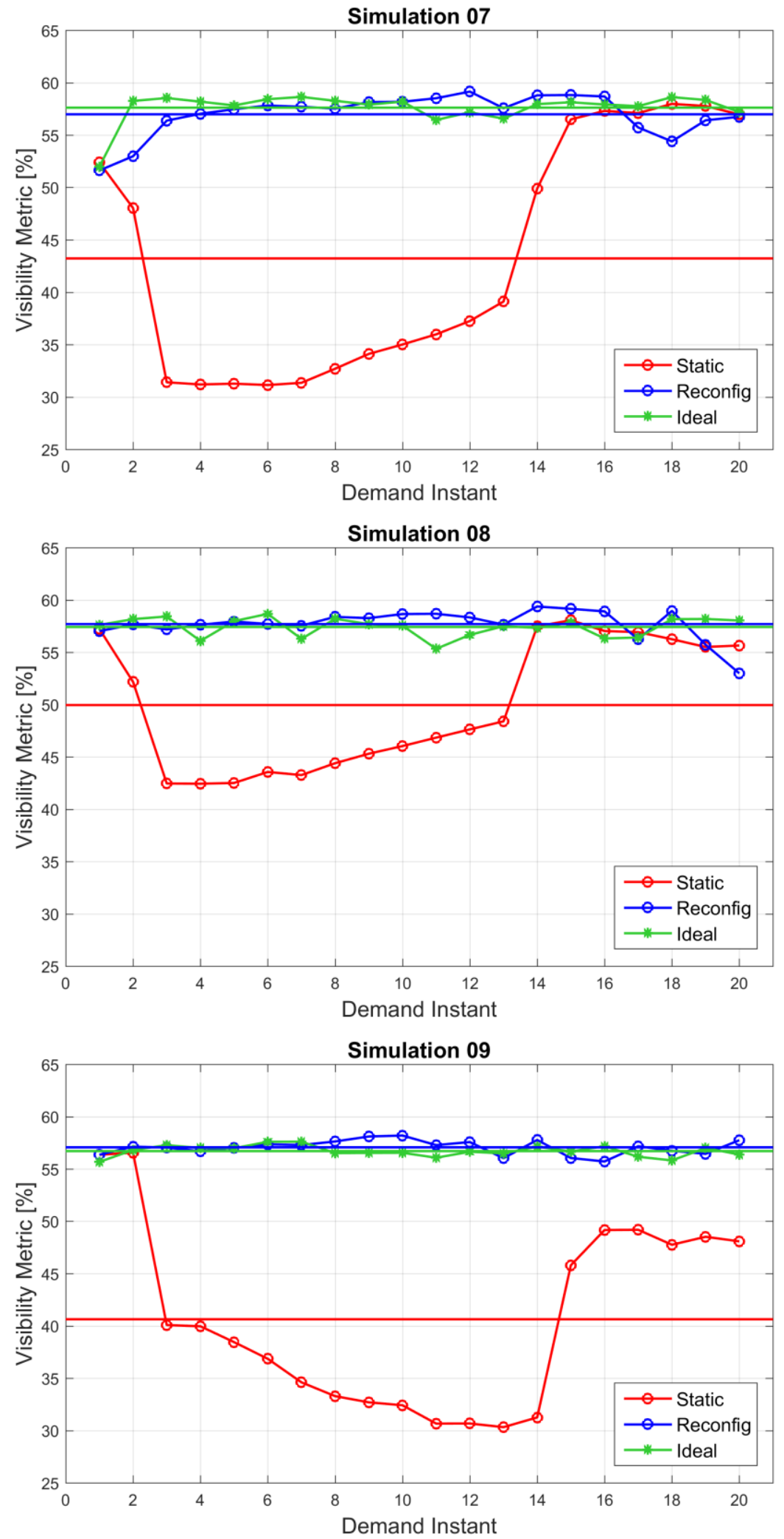

Figure 6.22: Surface area results for Simulations 7-9. 

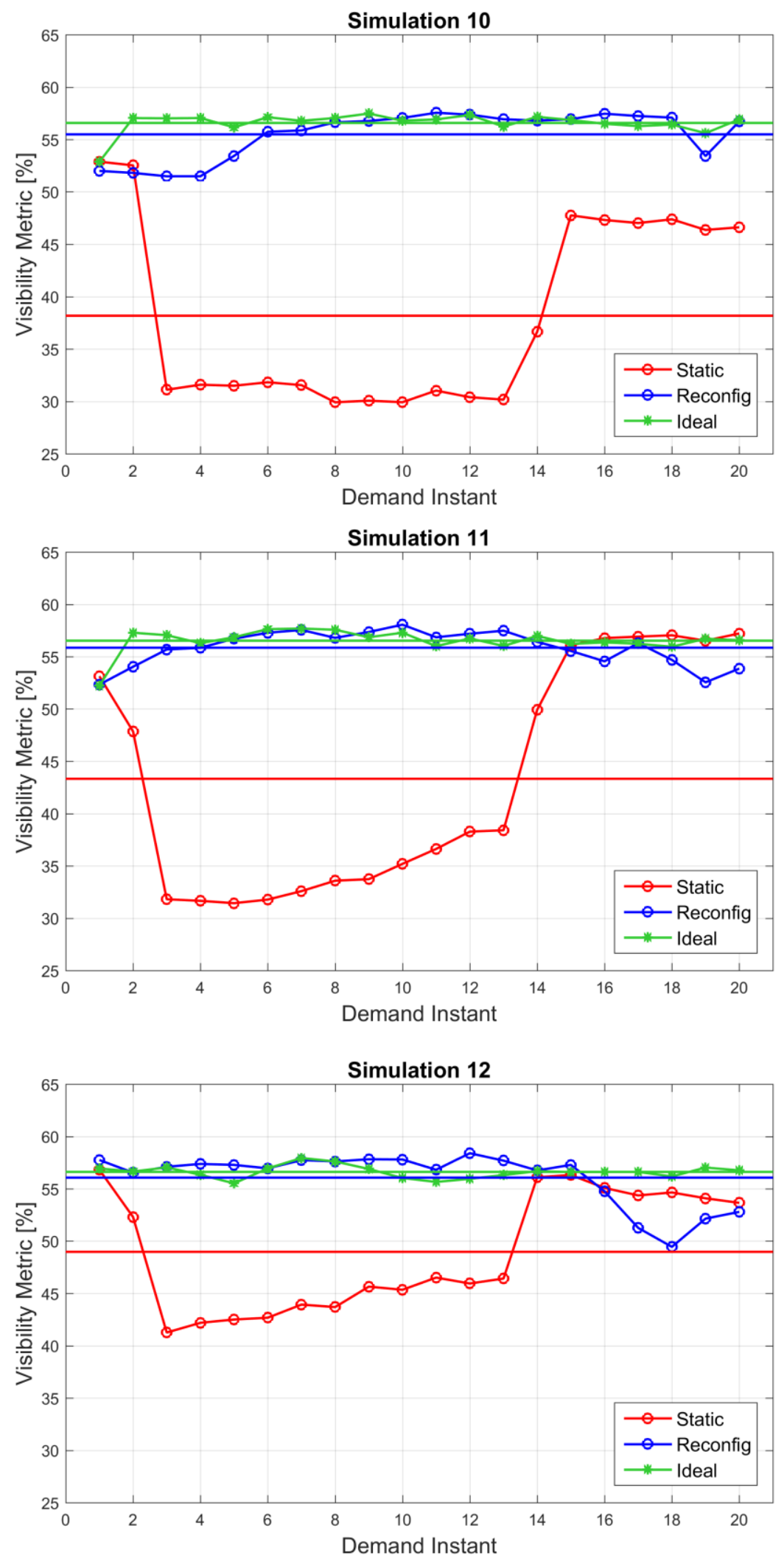

Figure 6.23: Surface area results for Simulations 10-12. 


\section{Chapter 7 \\ Experiments}

Experiments were conducted to validate the complete system in real-world conditions for shape recovery of an a priori unknown deforming objected through a multi-camera active-vision system. The experimental setup consisted of a set of fully calibrated Canon DSLR cameras mounted on linear and rotary actuators about a deforming robot. The experiments compared the results of the reconfiguration system developed to the application of static cameras, demonstrating the clear advantage of reconfiguration.

\subsection{Overview}

The purpose of the experiments was to validate the methodology through controlled experiments that simulate real-world operating conditions. The aspects of the experiments that were explicitly controlled included the position and orientation of the cameras, camera calibration and the deformation of the robot. The real-world conditions included uncontrolled lighting and the manufactured surface texture of the target object.

Camera calibration was conducted using the MatLab toolbox developed in [196]. The experimental setup consisted of a single computer with multiple linear and rotary actuators controlled by two separate controllers. Four Canon Rebel T3i and two Canon Rebel T5 cameras were used. The target object chosen for the experiments was a PLEOrb pet robot. The background separation process consisted of a GrabCut algorithm [197]. The experimental performance evaluation metric was based on the visibility metric introduced in Section 6.7.2.

A total of 10 experiments were conducted in sets of 2, for a total of 5 experimental sets. Each set consisted of a reconfiguration experiment, and a static experiment. Due to the limited DOF of each camera and fixed-focus calibration, the reconfiguration was limited to circumferential motion about the target object at a fixed radius. This limitation ensured the maximum DOF range at a given focal length for all cameras about the target object. The obstacle was added during run-time in MatLab directly following image acquisition to simplify the background separation process.

\subsection{Camera Calibration}

Camera calibration consisted of focus calibration and intrinsic and extrinsic parameter calibration. Focus calibration ensured that the camera's focus distance was consistent with the focus motor. The intrinsic and extrinsic camera calibrations produced the projective matrices necessary for triangulation and backprojection. Both types of cameras used an APC-S sensor that is $22.3 \times 14.9 \mathrm{~mm}$ in size with a maximum 
resolution of $5184 \times 3456$ pixels. All cameras used the same Canon EF-S 18-55mm f/3.5-5.6 IS lens set to $18 \mathrm{~mm}$ focal length.

The cameras were connected to a computer through USB and controlled via $\mathrm{C}++$ program implementing the Canon Electo-Optical Systems Digital Software Development Kit (EDSDK). The EDSDK provides function to connect Canon cameras to a host computer. Some example functions include camera image capture settings, focus motor control, image capture, and image transfer. The EDSDK allows the system automate the camera image capture and transfer process during runtime. The focus point of each camera can be set through the host computer by controlling the motion of the focusing servo incremented by steps. Manual focus control is important to maintain calibration parameters.

\subsubsection{Focus Calibration}

The calibration of the camera's focus point ensures captured images are sharp in the region of the target of interest. The sharpness of the image will dictate the number and quality of unique-key features detected. Blurry, out-of-focus images will produce less features, resulting in poor shape recovery accuracy. Therefore, the camera's focus must be calibrated, and controlled through the experiments to ensure the highest possible shape recovery accuracy.

The focusing servo is addressed through the EDSDK by step sizes, without an explicit numeric control. This means that the focus range must be heuristically determined by comparing the focus range to the number of steps taken by the motor. To map the focus distance, the camera calibration setup in Figure 7.3 was used. The camera's focal length was set of $18 \mathrm{~mm}$, and the focus servo stepped to the 'zero' position, namely, the focus motor was stepped out until it reached its limit. Then, the smallest increment steps were taken from the 'zero' position until the checkerboard image came into focus. An automated focus calibration method will be proposed in Chapter 8 . The resulting focus calibration was fit with a third-order polynomial, Figure 7.1. The camera parameters were set based on Table 7.1. The aperture was set based on the shutter speed to ensure images were not over-exposed.

Table 7.1: Camera settings.

\begin{tabular}{|l|c|l|}
\hline Property & Value & Reasoning \\
\hline Focal length & $18 \mathrm{~mm}$ & Maximize field of view and depth of field. \\
\hline ISO & 400 & Image noise reduction at given shutter speed. \\
\hline Shutter Speed & $1 / 50 \mathrm{~s}$ & Set to balance light capture based on aperture and ISO values. \\
\hline Aperture & $\mathrm{f} / 3.5$ & Smallest achievable aperture to maximize depth of field. \\
\hline Resolution & $5184 \times 3456 \mathrm{px}$ & Maximize image detail. \\
\hline
\end{tabular}




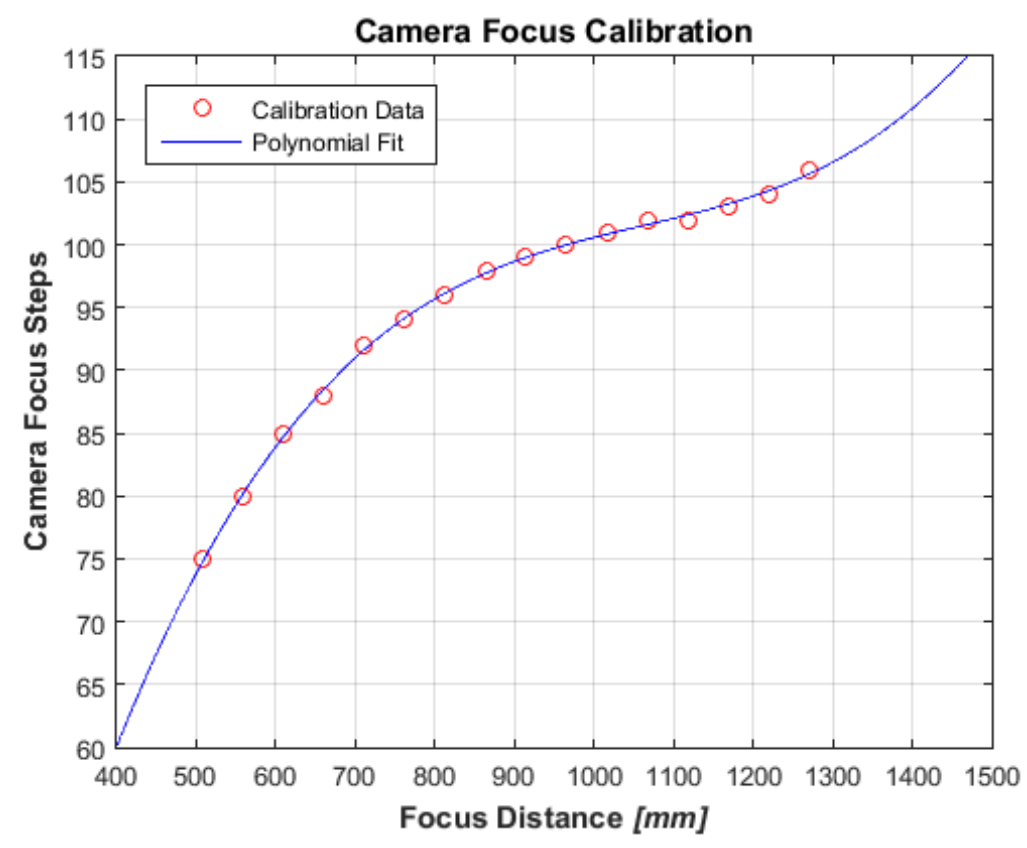

Figure 7.1: Sample camera focus calibration.

The depth of field, or DOF of a camera represents the range of distances from the camera that are considered in focus based on the hyperfocal distance, and circle of confusion value. The circle of confusion references to the smallest diameter of a point of light than can be resolved by the camera's sensor, ensuring the point is in focus. Common values for the circle of confusion diameter range from $0.018-0.025 \mathrm{~mm}$. A value of $0.018 \mathrm{~mm}$ was chosen for the circle of confusion to produce the minimum expected depth of field as the worst-case scenario. The depth of field at each focus distance could then be approximated by the following equations:

$$
h_{f}=\frac{f^{2}}{a c_{o}} \text {, }
$$

where $h_{f}$ is the hyperfocal distance, $f$ is the focal length of the lens, $a$ is the $f$-number or aperture, and $c_{o}$ is the circle of confusion size. Then, the near and far focus distances, $d_{\text {near }}, d_{\text {far }}$, are calculated:

$$
\begin{gathered}
d_{\text {near }}=\frac{h_{f} d_{\text {focus }}}{h_{f}+d_{\text {focus }}-f}, \\
d_{\text {far }}=\frac{h_{f} d_{\text {focus }}}{h_{f}-d_{\text {focus }}-f},
\end{gathered}
$$

where $d_{\text {focus }}$ is the set focus distance on the camera. These equations produce the expected depths of fields represented by Figure 7.2 for an $18 \mathrm{~mm}$ lens. 


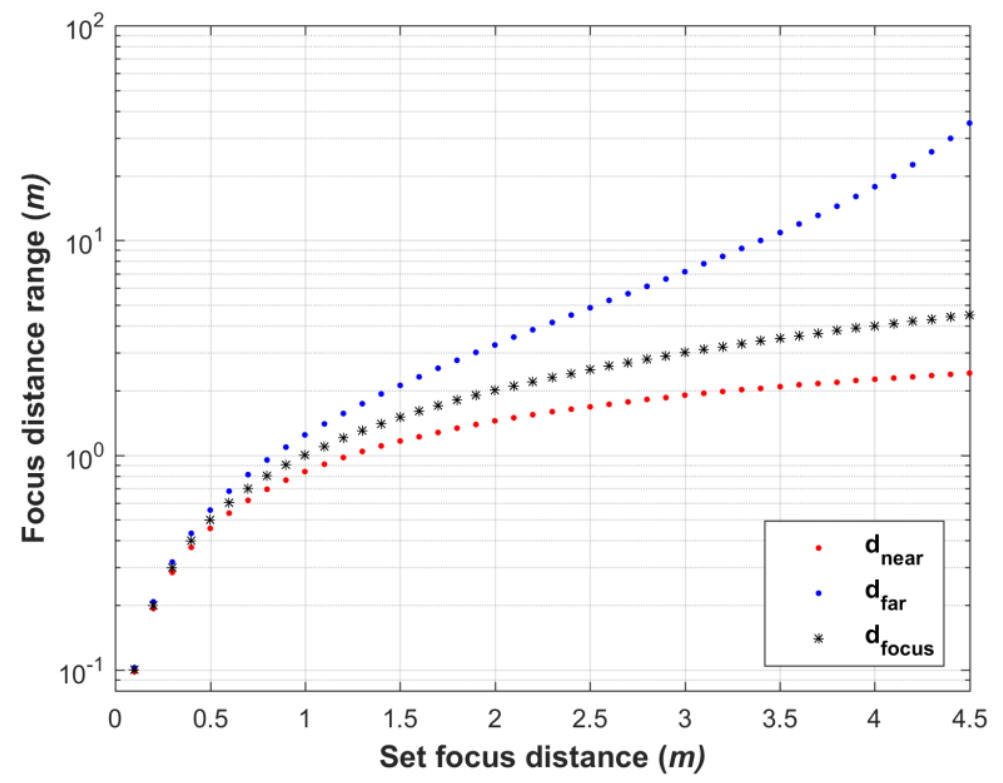

Figure 7.2: Depth of field range for $18 \mathrm{~mm}$ focal length lens.

\subsubsection{Parameter Calibration}

All six cameras were individually calibrated using the MatLab calibration toolbox [196]. The camera calibration setup is presented in Figure 7.3. The calibration setup consisted of an optical table assembly with a calibration plate, and the camera mount. A detailed view of the camera mount is presented in Figure 7.4. The camera calibration setup consisted of five reference frames; the world reference frame, W, the calibration plate reference frame $R_{p}$, the camera mount reference frame $R_{f}$, and the camera reference frame $\mathrm{R}_{\mathrm{c}}$.

The calibration toolbox produced an intrinsic calibration matrix, and an extrinsic calibration matric for each calibration image taken. A set of 100 calibration images were taken for each camera with one fixed image using the calibration setup (Figure 7.3). The bulk of the images were used to calibrate the intrinsic parameters and one fixed image produced the extrinsic calibration matrix. The extrinsic calibration matrix referenced the camera's position and rotation relative to the calibration plate. Since the calibration plate, $\mathrm{R}_{\mathrm{p}}$, and the camera mount, $\mathrm{R}_{\mathrm{f}}$, were explicitly located in world coordinate space of the calibration setup, $\mathrm{W}$, each camera's reference frame, $R_{c}$, could be directly related to the camera's mounting plate, $R_{f}$. The camera mounting plate reference points were located on the top plane of the mounting plate centered about each camera's tripod mount. The mounting plates implemented a secondary fixing bolt to ensure that when the cameras were attached and removed, they would always orient to the same position. 


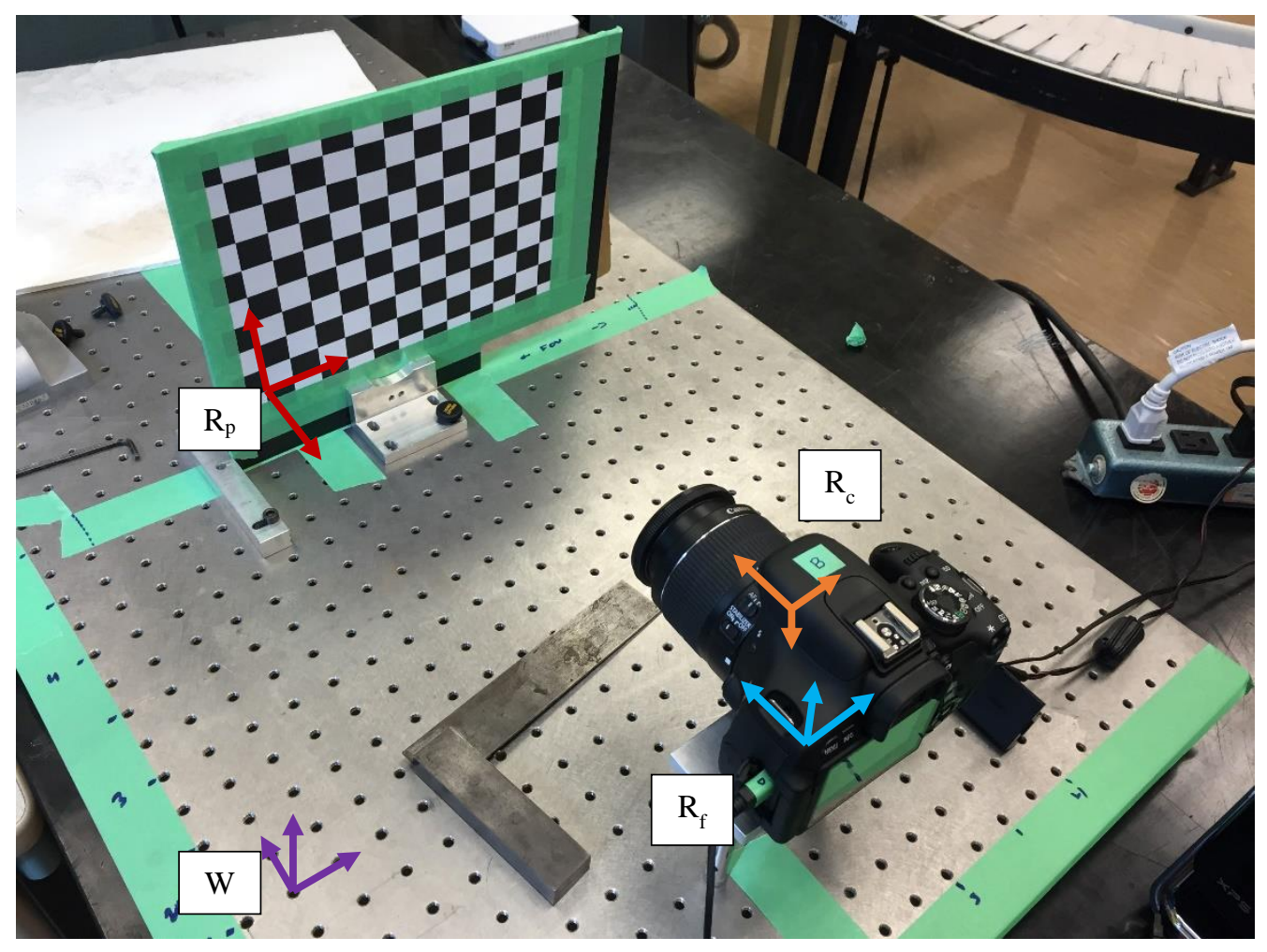

Figure 7.3: Camera calibration setup.

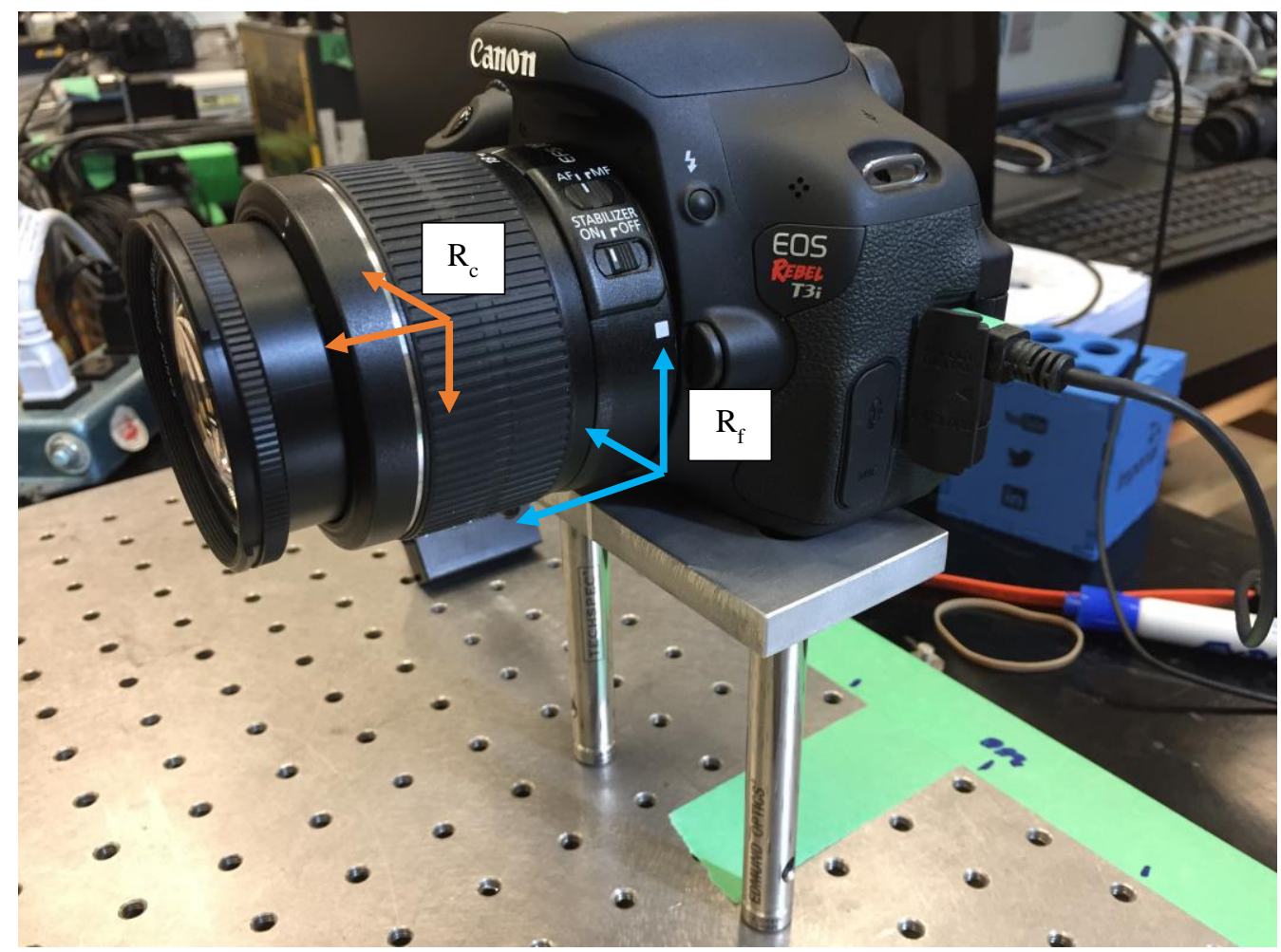

Figure 7.4: Camera mount detail view. 


\subsection{Experimental Setup}

The experimental platform consisted of six DSLR cameras, six linear actuators of varying lengths, six rotary platforms, 12 motor drivers, two motor controllers, one Intel i7 PC, and one robot representing the deformable object. The layout of the experimental setup is presented in Figure 7.5 with the cameras and robot highlighted. Four Canon Rebel T3i cameras were mounted on rotary platforms on top of 300mm linear actuators aligned to the y-axis. Two Canon Rebel T5 cameras were mounted on rotary platforms. The target object was mounted in the center of the workspace on a rotary platform that sat on top of $450 \mathrm{~mm}$ linear actuator along the $\mathrm{y}$-axis and a $200 \mathrm{~mm}$ linear actuator along the $\mathrm{x}$-axis.

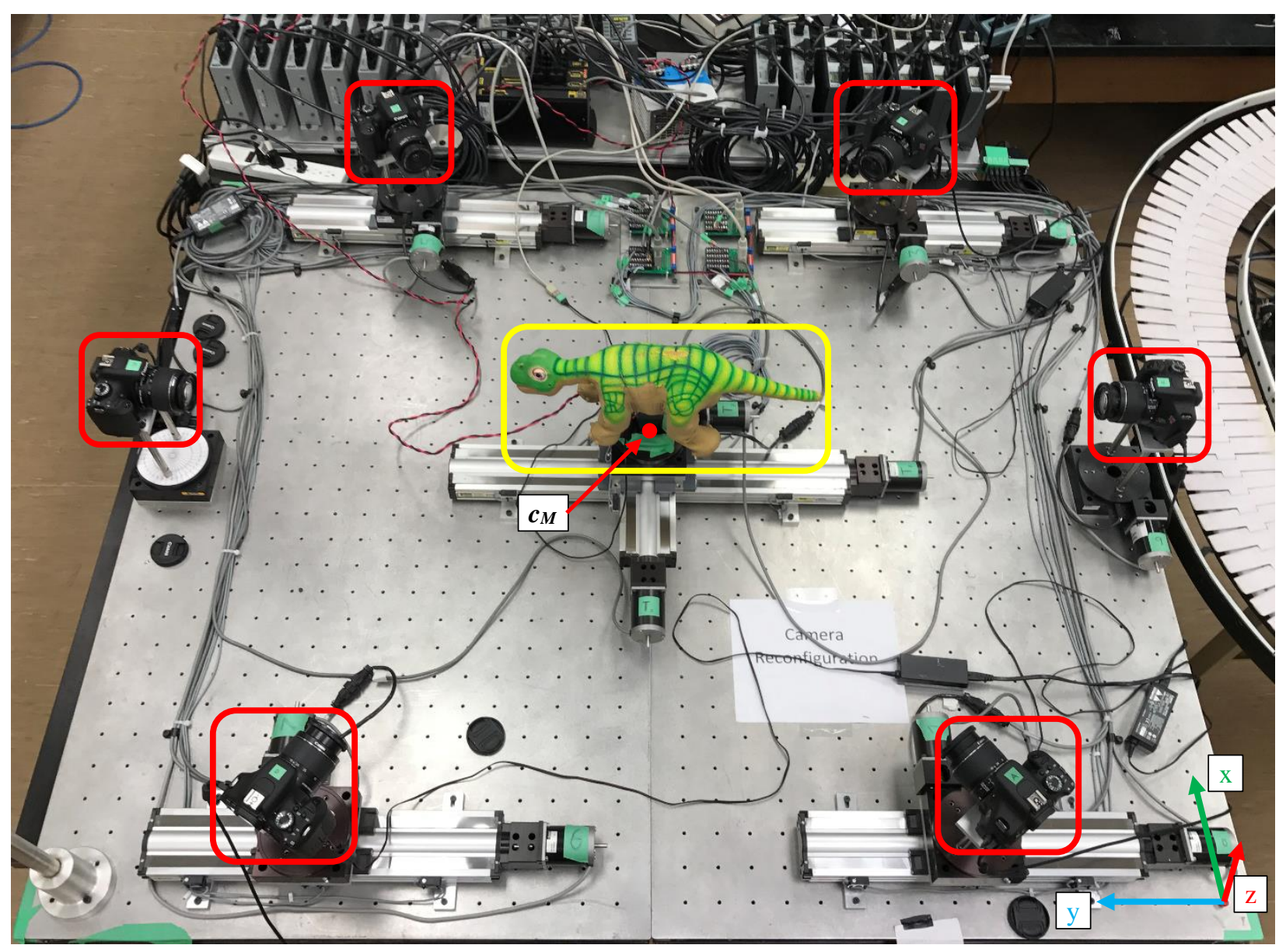

Figure 7.5: Experimental setup.

The cameras were circumferentially distributed about the target object in the workspace. The camera calibration was based on a fixed focal length and focus distance, thus, varying the focus distance of the cameras would require recalibration. Therefore, to ensure the target object was maintained in both the field of view and depth of field of all cameras, the camera motion was limited to a circle centered about the robot, $c_{M}$, at a radius of $775 \mathrm{~mm}$. 
The hardware and software design of the experimental setup is presented in Figure 7.6 and Figure 7.7, respectively. The hardware design consisted of two separate ParkerMotion 8 motor controllers communicating with a host PC over USB and Ethernet. The controllers were used as an interface between the host PC and each motor driver to ensure accurate positioning of all actuators. Each controller was connected to subset of the 13 total actuators through a motor driver. The motor drivers decoded the commanded position from the controllers and moved the stepper actuators to the desired positions. The global positions of all actuators were measured from their homing limit switches relative to the workspace coordinate frame denoted by the axes in Figure 7.5. The controllers and six cameras were controlled through a custom-written $\mathrm{C}++$ program, CommandControl, Figure 7.7.

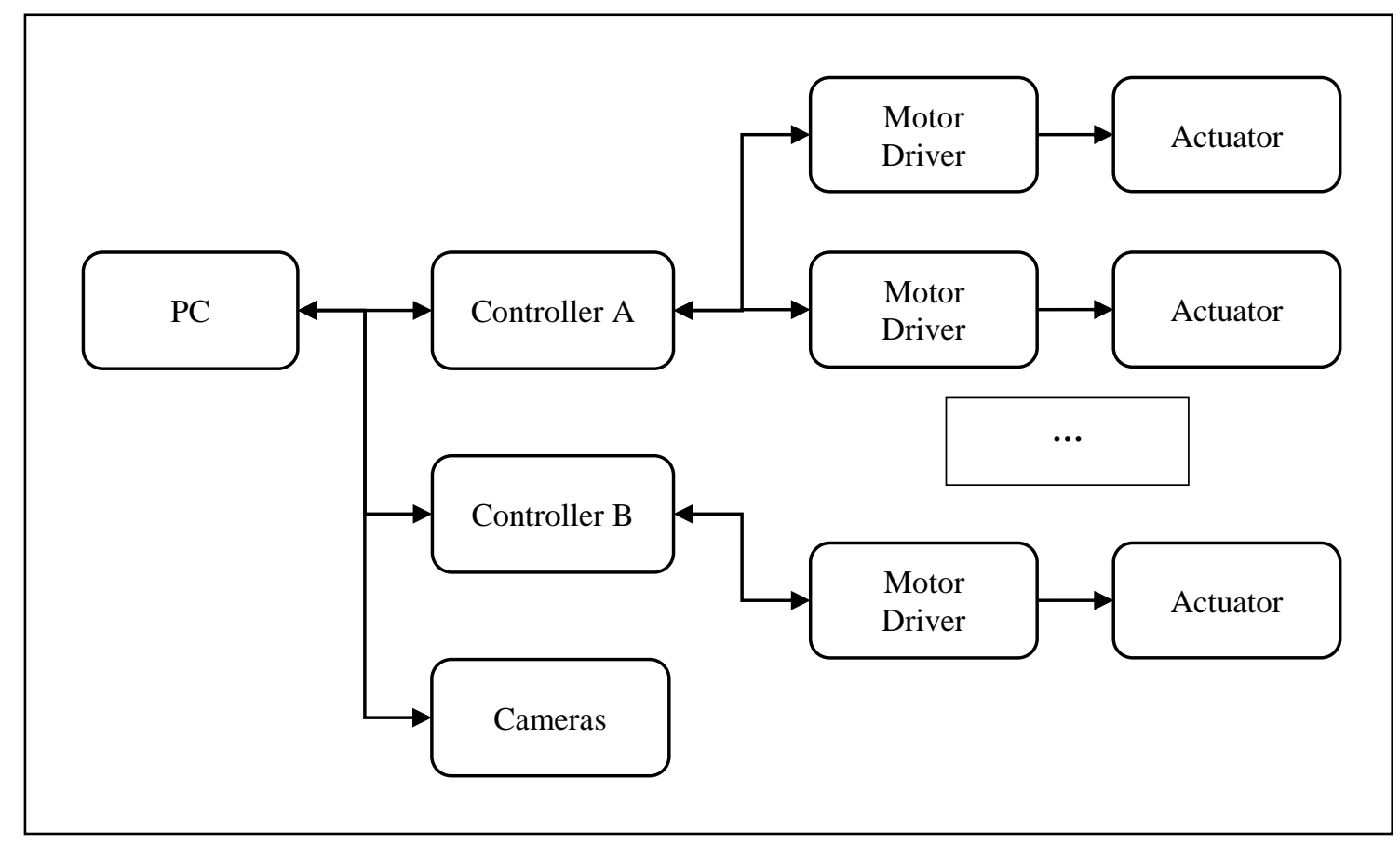

Figure 7.6: Hardware implementation of the experimental setup.

The CommandControl software packaged the necessary communications between the host PC, the controllers and the cameras through USB, Ethernet, and the EDSDK. The software utilized standard Microsoft Serial and Socket communications for USB and Ethernet communication. A set of functions were written for setting parameters and parameter settings queries. Camera-based functions included setting camera focus through based on the focus calibration, shutter speed control, aperture control, and ISO setting control. The photo taking function triggered the camera's shutter and stored the image in the camera's local memory until a retrieve function downloaded the image from the camera to the host PC. Querying camera parameters allowed the host to verify camera settings. 
Motor control was handled by custom-written C++ libraries based on ParkerMotion software commands. Each controller implemented a separate communication protocol based on the physical port available on the controller. The CommandControl software was capable of uploading custom programs to each controller that consisted of initializing motor settings such as velocity, acceleration and jerk, and motor homing functions. Further, automated query functions were deployed to ensure the host PC received up-todate information regarding motor position. The main code was developed in MatLab as with the simulations. The obstacle was digitally inserted in the 3D space during MatLab shape recovery to ensure accurate placement of the obstacle. This overcame the problem of adding additional motorized platforms responsible for obstacle placement.

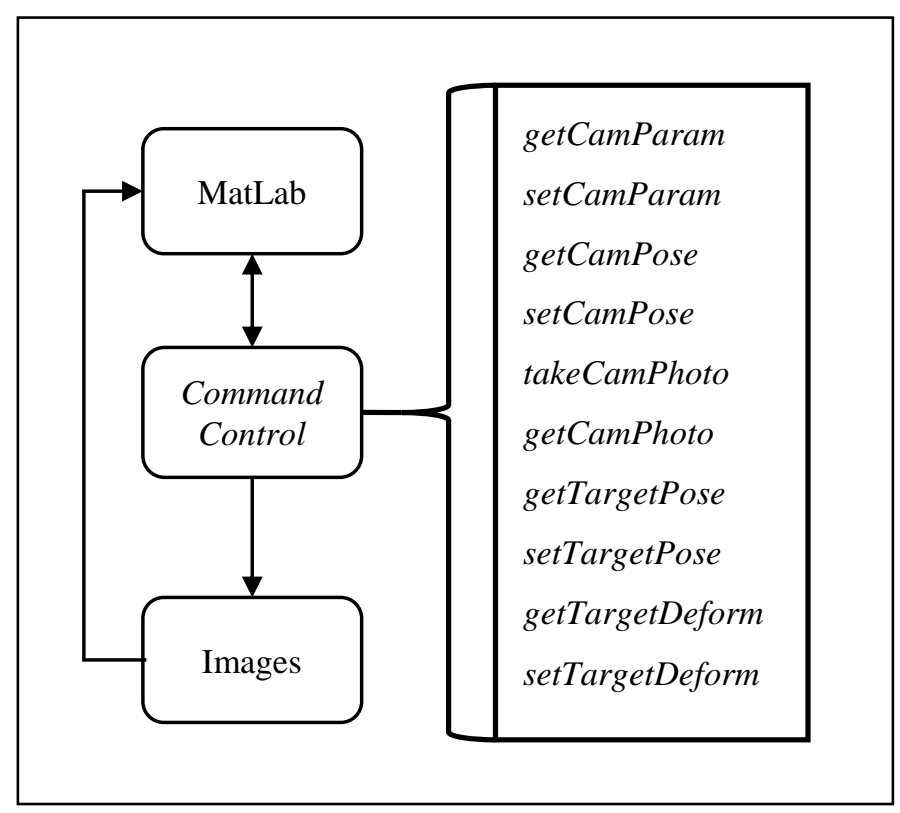

Figure 7.7: Software implementation of the experimental setup.

The target object chosen for the experiment was the PLEOrb robot, Figure 7.8. The robot is composed of 15 servo motors actuating the mechanical armatures with a patterned texture rubber skin. The servo's positions can be directly controller through USB serial interface. A control program package was written and integrated into CommandControl for automatic USB connection to the robot and servo position setting and querying. The explicit servo control enables repeatability in positioning the robot's armatures. The surface texture was tested for SIFT feature density and matching, at the maximum image resolution. The variance of the surface's texture produced many features uniformly distributed over the visible surface. An example of the feature density recovered in presented in Appendix E. The volume and surface area of the robot were calculated manually by measuring each limb individually and approximating limbs with geometric objects such as cylinders, cones, and rectangles. 


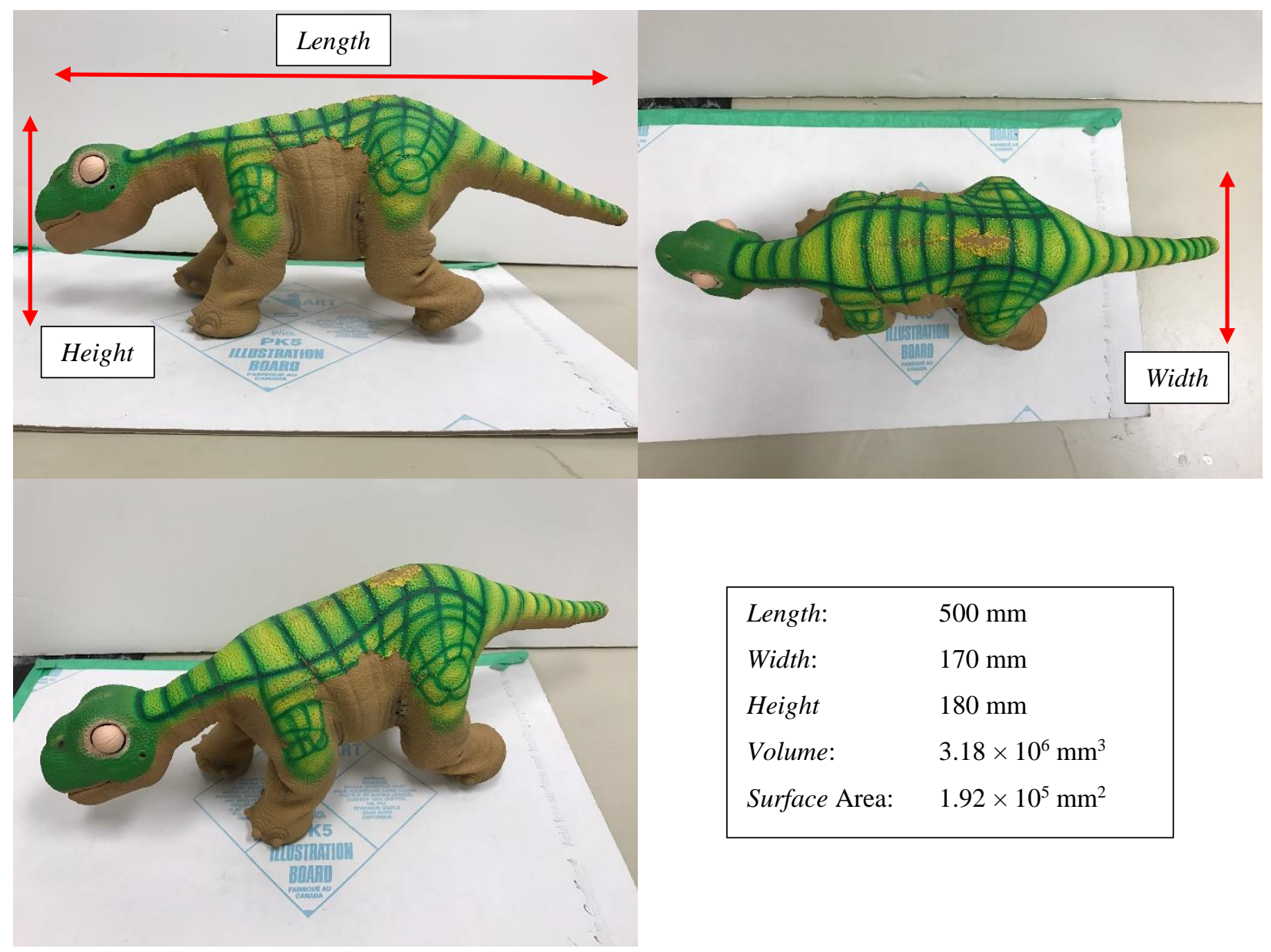

Figure 7.8: PLEOrb Robot, properties.

\subsection{Background Segmentation}

Background segmentation is outside of the scope of this dissertation, yet, it is a necessary aspect of the system for correct shape recovery. The difficulty of developing a generic background segmentation method is based on the open-ended problem of target selection. Within the context of this work, target selection was identified as an application-specific task and must either be developed or implement for specific tasks. For example, user-assisted target selection would greatly simplify background segmentation as the system would rely on the a priori user provided target data to segment the target from the background. Recognition based systems could provide initial segmentation estimates by attempting to recognize the target object from a bag-of-words descriptor [1]. Thus, the background segmentation method relies greatly on the target selection method, and for the purposes of this work, a user-assisted target selection and segmentation method was implemented. 
The segmentation process was carried out during run time through a user-guided GrabCut operation [197]. The GrabCut method is based on the graph cut energy minimization problem solved via maximum flow through a graph. The GrabCut process consists of the user providing a set of guide points on the input image that are near the target's boundary. The algorithm then solves for the image boundary that optimizes the max-flow min cut problem. The output of the algorithm is a binary image mask that labels the pixels as background or target, Figure 7.9.

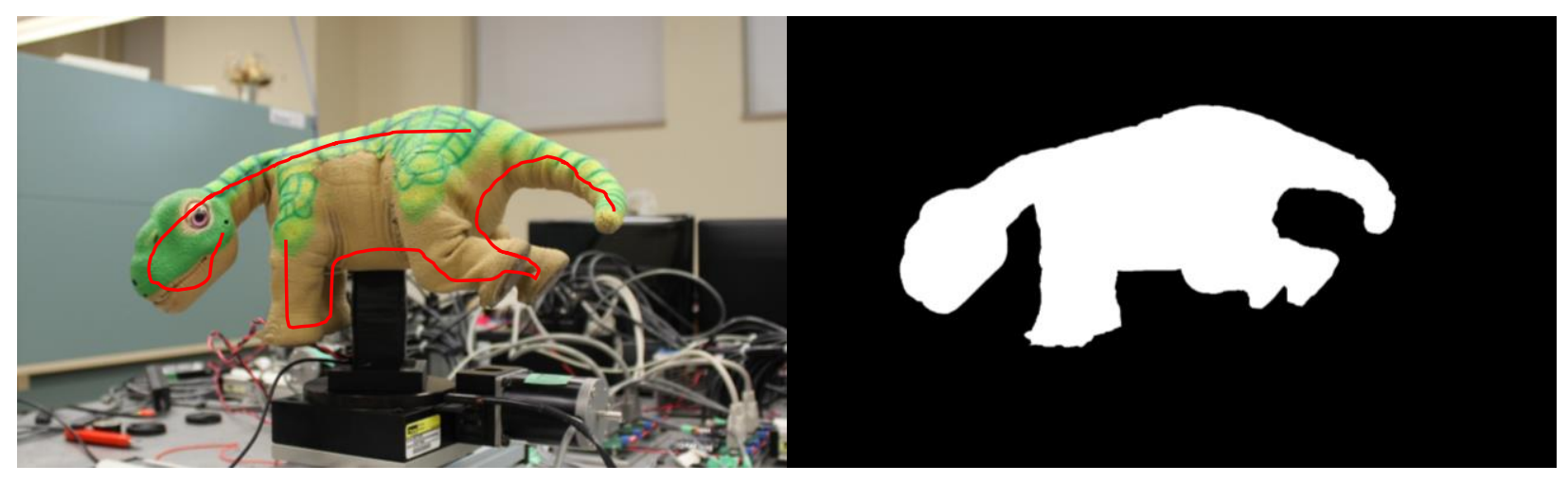

(a)

(b)

Figure 7.9: Background segmentation through GrabCut, left: input image with guides, right: resulting binary segmented image.

\subsection{Errors}

The experimental errors used to validate the methodology were similar to the simulation errors. One difference between simulations and experiments is the lack of an a priori known target model for the latter. Specifically, in simulations, the target object's model was explicitly known outside of the system and could be used to directly analyze the shape recovery accuracy and completeness. Further, in the complete system simulations, Section 6.7.3, the true object's shape was used for the performance evaluation metric for surface area visibility. In contrast, the true object's 3D model that represented the exact surface deformation of the target object was available, since the exact deformation of the surface (i.e.: folds and creases) could not be prediction through a 3D model. Therefore, the surface-based performance evaluation metric presented in Equation (6.8) is modified to measure the surface area recovered relative to the fixed surface area of the target object:

$$
\eta_{s y s}^{*}=\frac{\beta_{v i s}}{S_{\text {model }}},
$$


where $S_{\text {model }}$ is the fixed surface area of the model.

Another difference between the experimental and the simulation evaluation metrics is that the simulation estimated stereo surface area visible based on the true object's model while the experiment estimated the stereo surface area visible based on the fused model. This area was, then, divided by the manually measured surface area of the robot to normalize the scores for all experiments.

\subsection{Results}

A total of five sets of experiments were conducted. Each set consisted of a static camera run, and a reconfiguration run. An ideal run could not be possible without an exact, 3D-actuated model of the robot with accurate surface deformation. Due to the fixed focus distance calibration of the cameras, the reconfiguration run was limited to radial placement about the center of the object. The static cameras were placed on a single plane oriented towards the center of the robot with a stereo disparity of $100 \mathrm{~mm}$, with a radial distance of $775 \mathrm{~mm}$ about the center. Each stereo-pair was separated by $120^{\circ}$ from the other two for the static experiments, and as an initial placement for reconfiguration. The static camera placement was used as the initial camera placement for the reconfiguration run. Each experimental run consisted of the robot deforming all its joints over a period of 20 demand instants, and an obstacle moving to occlude one set of static cameras. The obstacle was added in MatLab, in the 3D space on-line during processing, allowing precision placement in the workspace.

The results of the experimental sets, in terms of the performance evaluation metric from Equation (7.4), are presented in Figure 7.10 and Figure 7.11, wherein the solid lines represent the average performance metrics for the complete set. The experiments indicate a clear performance advantage with stereo-camera reconfiguration compared to static cameras in the presence of workspace obstacles. Every static experiment experienced an approximate $20 \%$ decrease in surface area visibility due to the occlusion when compared with the system developed. The developed system could maintain a consistent surface area visibility throughout the entirety of each experiment.

A bird's-eye-view of a static-camera experiment from each odd frame is presented in Figure 7.12 and Figure 7.13. The images illustrate only the recovered surface from the complete model recovered to illustrate the performance metric, namely, only the stereo visible surface patches of the recovered modelled were filled in for animation. Complete filled polygon illustrations are provided in Appendix G. The overhead view provides the most informative viewpoint of the stereo-triangulated portions of the objects recovered. The shape recovery sequence for the static camera resulted in a large loss of surface area visibility due to the occlusion. In contrast, the reconfiguration sequence, as seen in Figure 7.14 and Figure 7.15 maintained surface visibility during the obstacle's motion by moving the stereo camera pairs around 
the obstacle. Further, the non-occluded cameras were also reconfigured, as the reconfiguration optimization routine expected improved surface visibility depending on the expected object's deformation. Movie-strip representations of the experiments are presented in Appendix F.
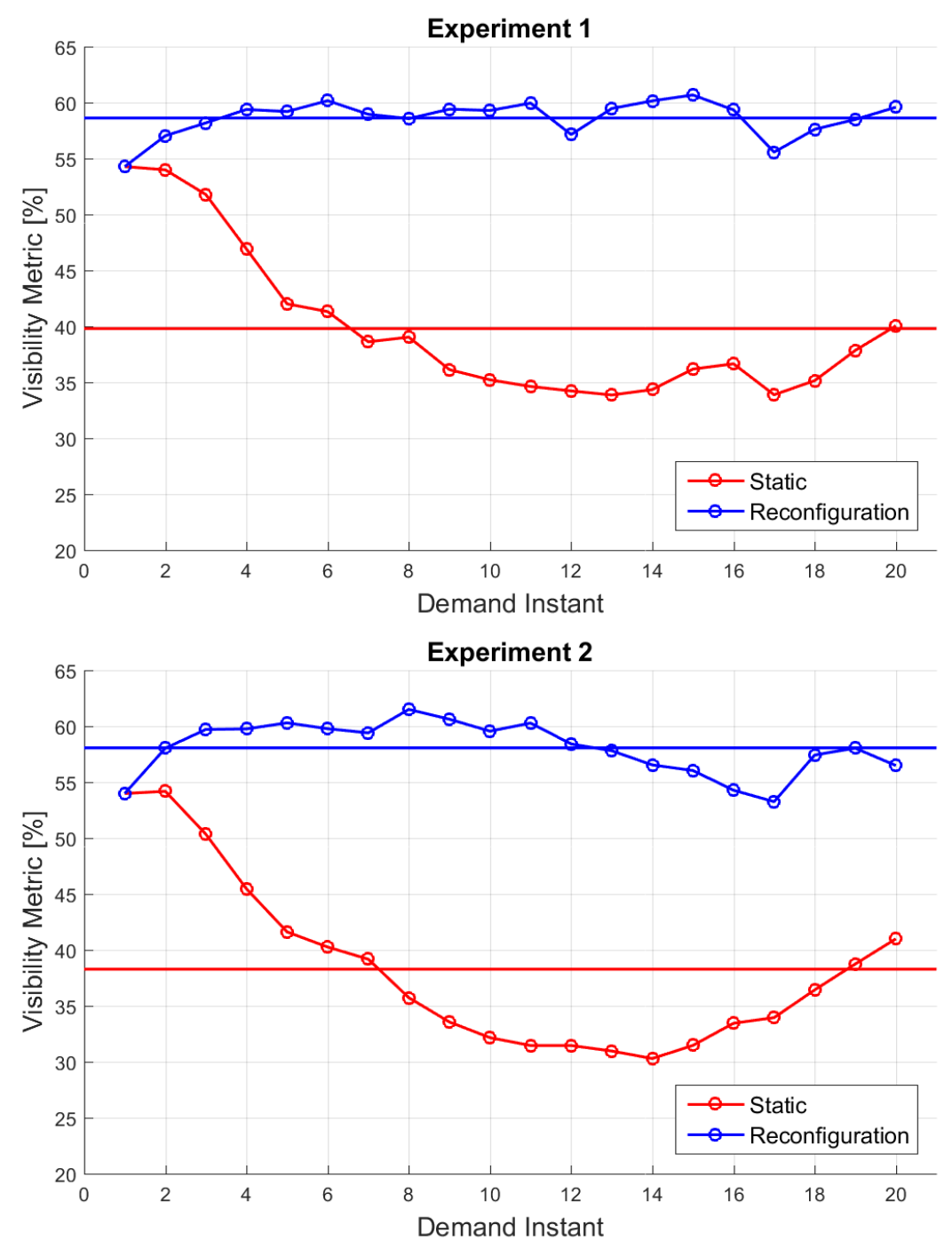

Figure 7.10: Results for Experiments 1-2. 

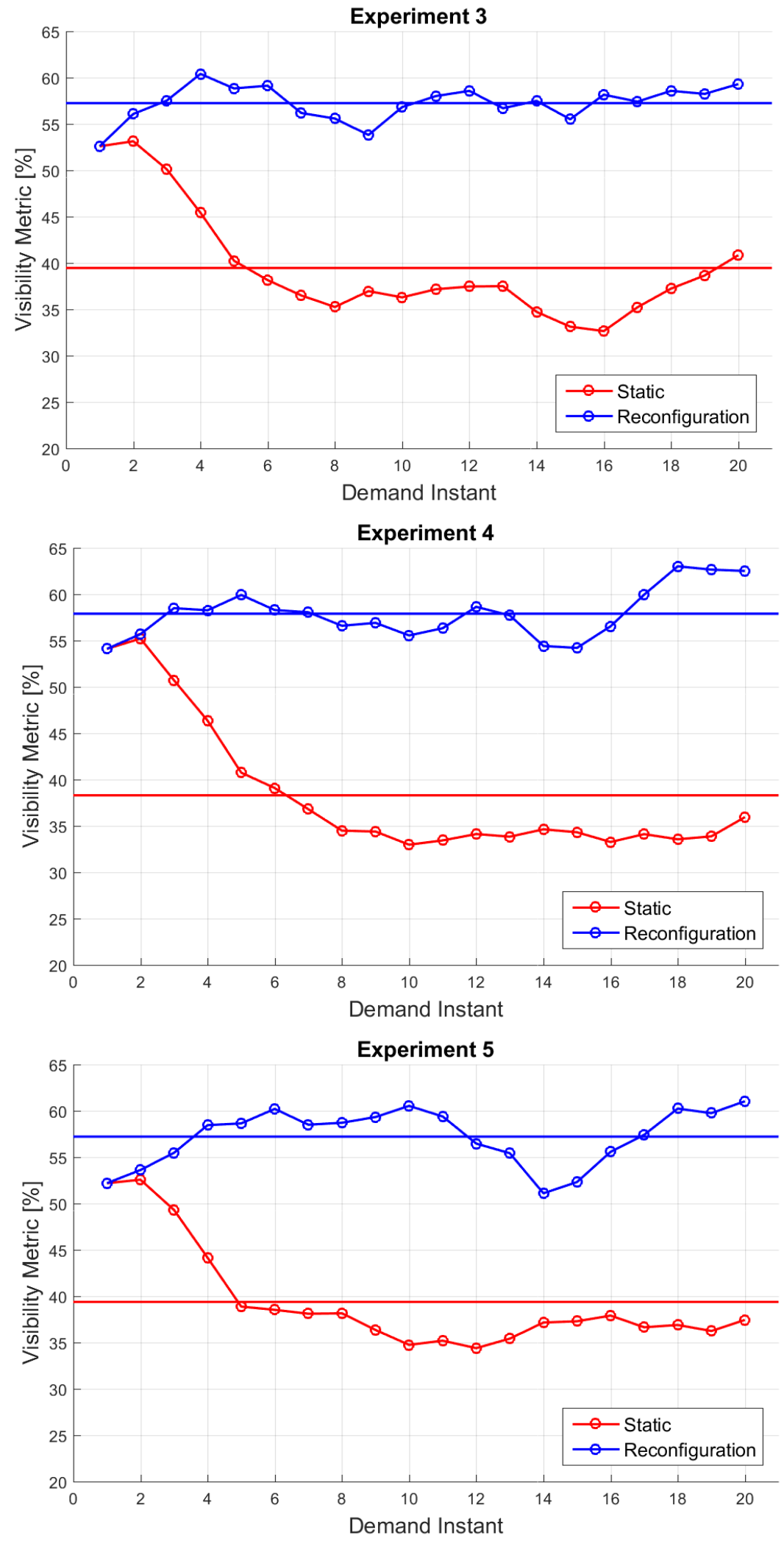

Figure 7.11: Results for Experiments 3-5. 

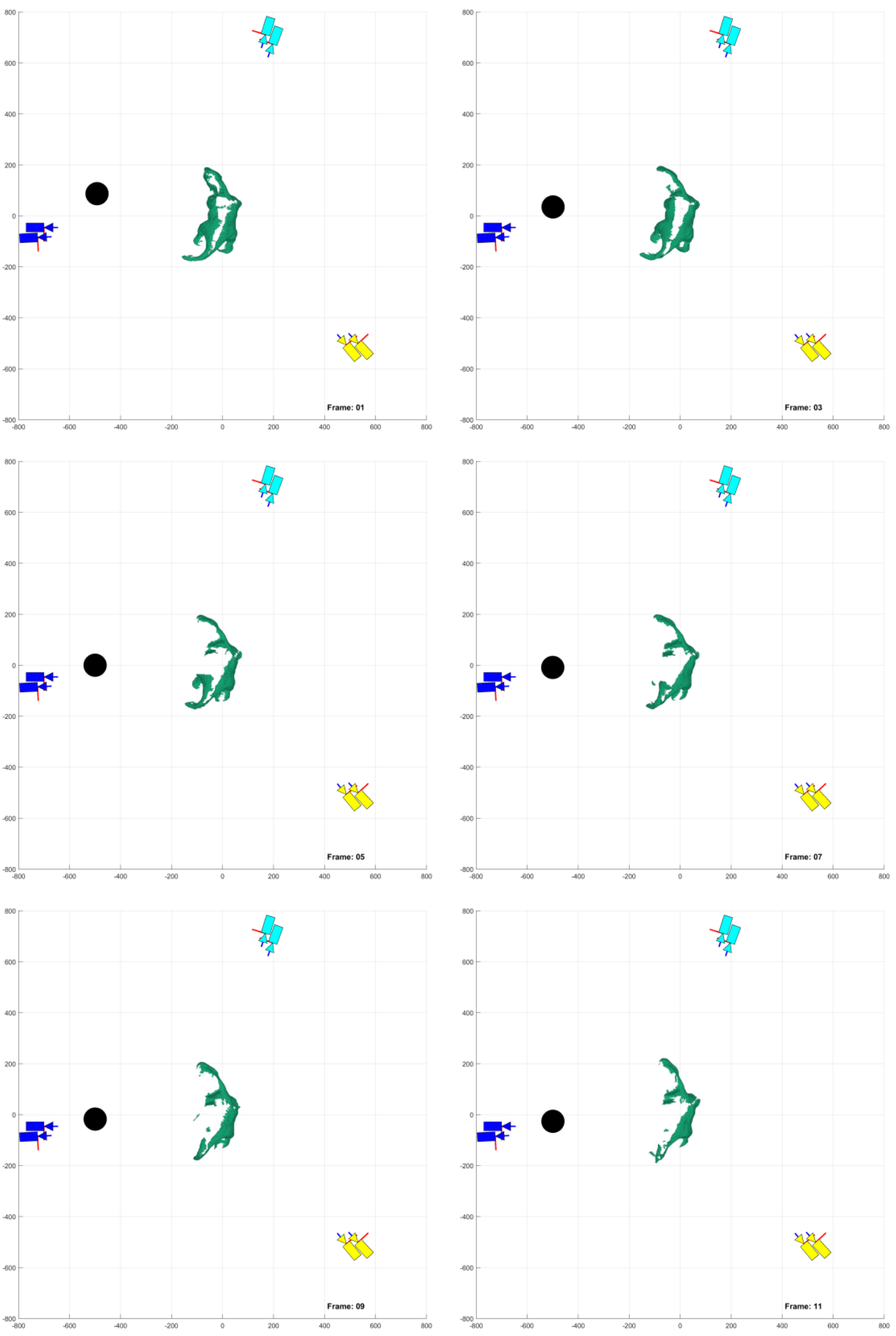

Figure 7.12: Static camera experiment, Simulation Frames $\{1,3,5,7,9,11\}$, respectively. 

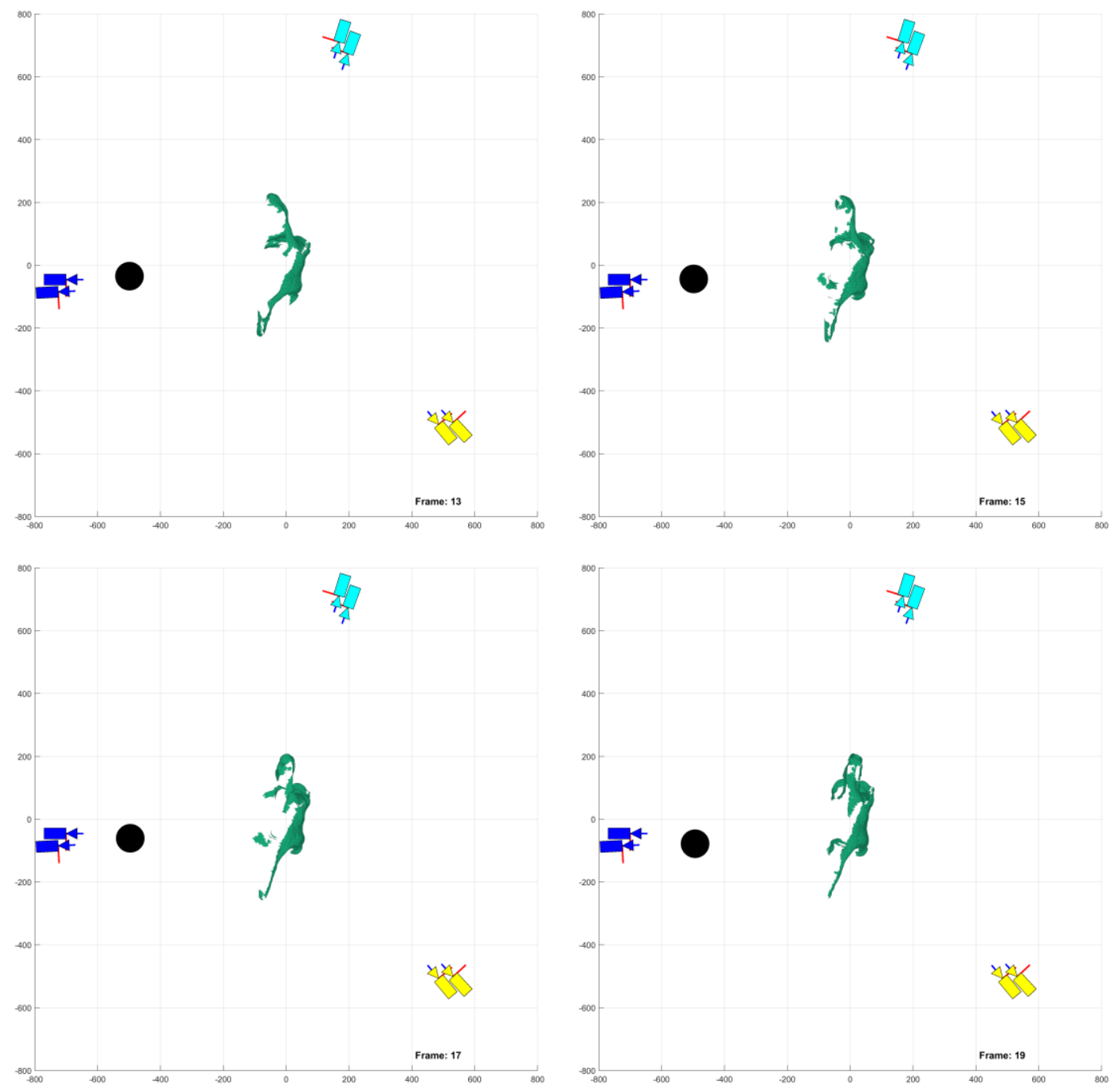

Figure 7.13: Static camera experiment, Simulation Frames $\{13,15,17,19\}$, respectively. 

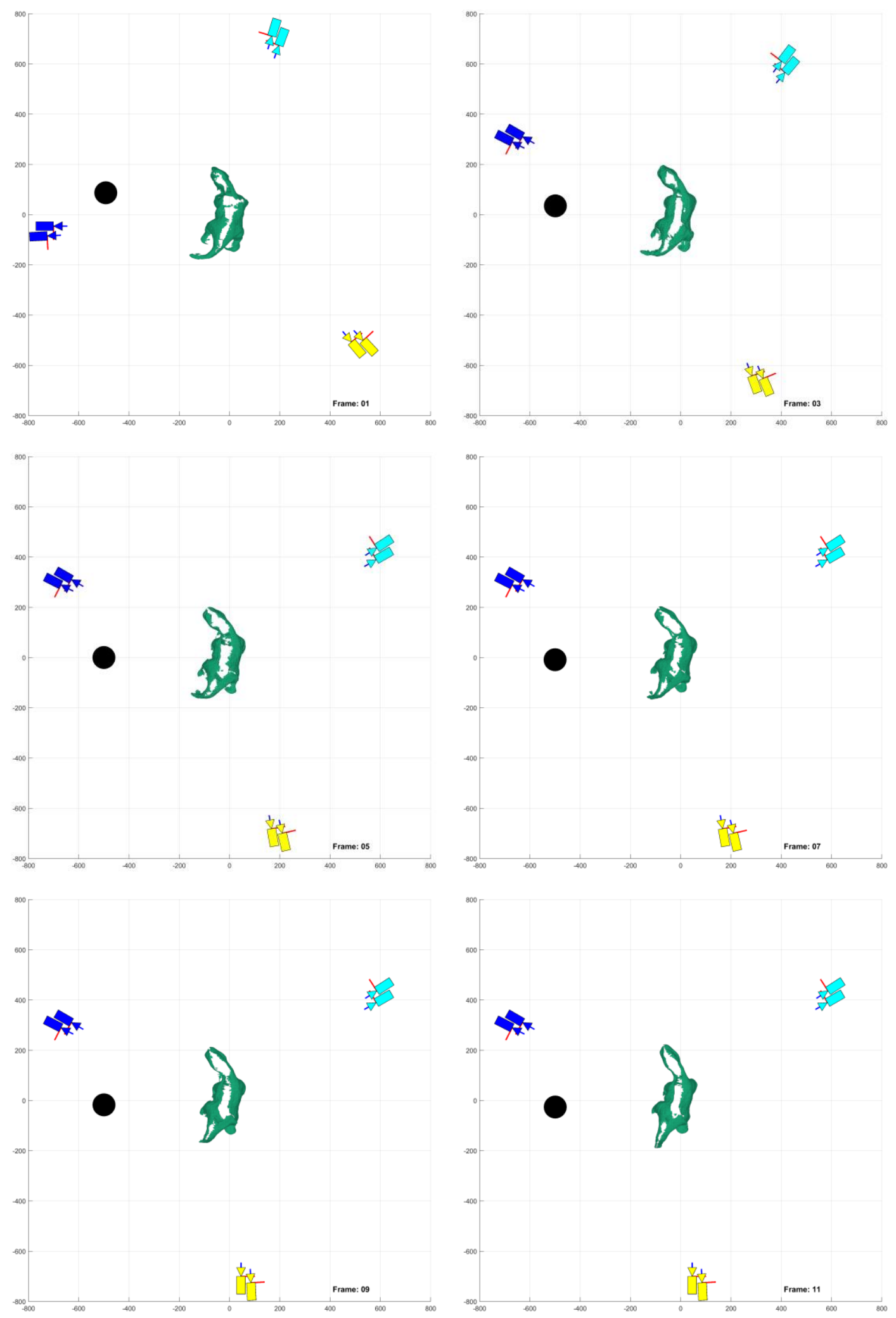

Figure 7.14: Reconfiguration experiments through proposed method, Simulation Frames $\{1,3,5,7,9$, 11 \}, respectively. 

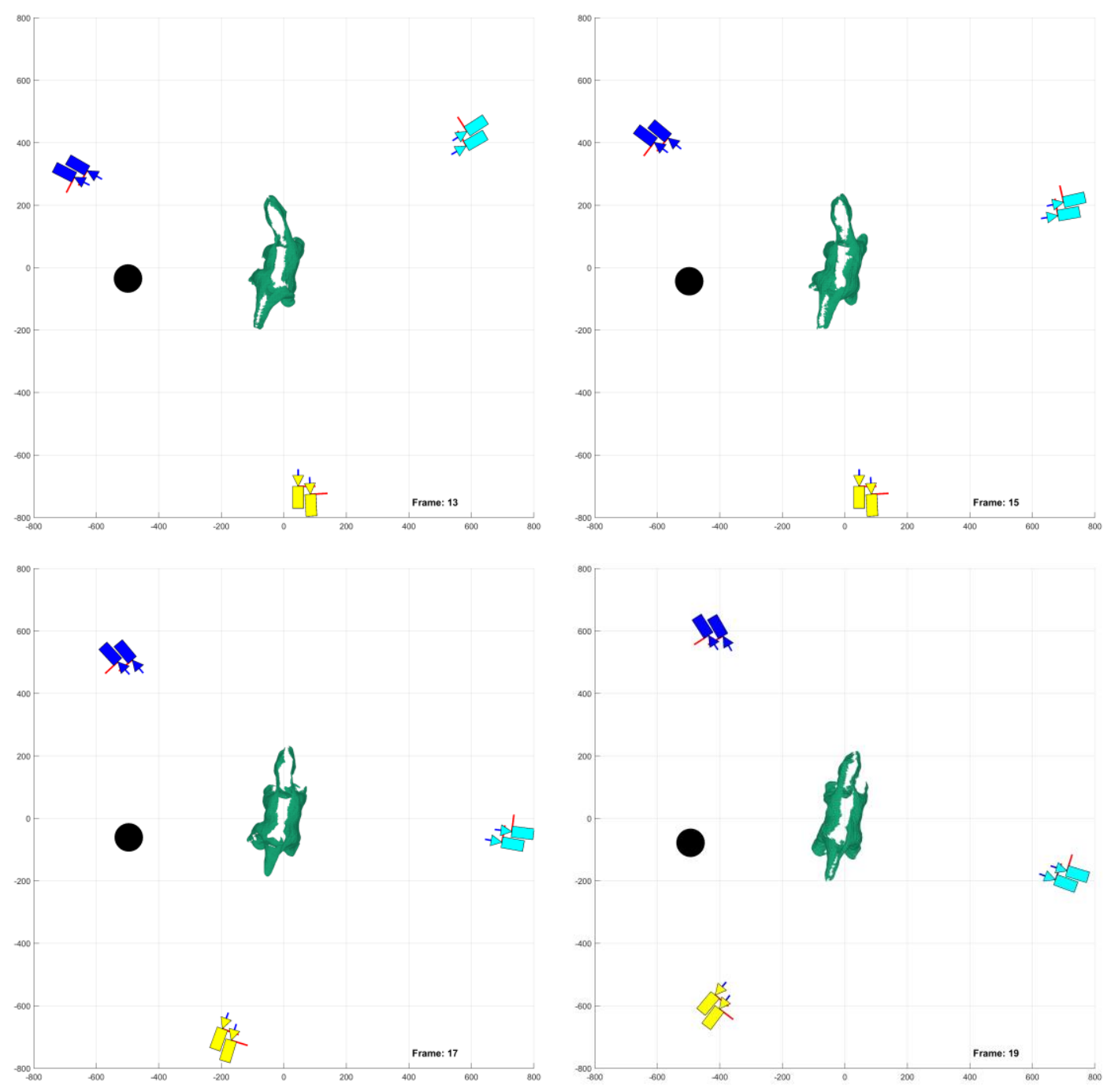

Figure 7.15: Reconfiguration experiments through proposed method, Simulation Frames $\{13,15,17,19\}$, respectively. 


\section{Chapter 8 \\ Conclusions and Future Work}

This dissertation presented a modular, on-line methodology for the shape recovery of a priori unknown deforming object through a multi-camera active-vision system. The proposed methodology offers a generic approach to the shape recovery problem by framing the problem as a surface visibility maximization. This generalization makes the method applicable to a wide variety of shape recovery problems that have, in the past, been approach through ad hoc solutions. The methodology consists of three tasks: model generation, deformation estimation, and reconfiguration. Each task was developed as a novel component of the complete shape recovery system. The complete system was tested in Chapters 6 and 7 through simulations and experiments that demonstrated the system's capabilities under both controlled, and real-world conditions. The final chapter of this dissertation with review and summarize the complete system, and then discuss potential future research.

\subsection{Summary and Conclusions}

The objective of this dissertation was to develop a generic system for shape recovery of a priori unknown deforming objects. A priori unknown objects were defined as a homogenous solid who's 3D geometry is unknown to the system. Deformation was defined as the motion of the object's surface relative to the center of the object's center of mass over a set of discretized demand instants. Thus, the objective of the system was to recover the 3D shape of the deforming object such that a quality metric was maximized. A system capable of accurate shape recovery of any arbitrary object can be classified as a generic system. Unlike $a d$ hoc methods, generic systems can recover the shape of arbitrary objects without an a priori known model that guides the recovery process. The motivation behind generic systems is based on their robustness, applicability and ease of implementation.

The applications of a generic shape recovery system include health-care, surveillance, and entertainment fields. A generic shape recovery system can greatly benefit many aspects of the health-care field including robot-assisted surgery, and medical rehabilitation. Robotic surgery may depend on shape recovery for accurate depth perception and modelling of various internal organs, thus a generic approach to shape recovery may handle the dimensional variance observed between patients better than an $a d$ method. Similarly, a generic shape recovery, such as a motion-capture, for medical rehabilitation would not require a full patient scan to produce a 3D model. Thus, each patient's model would be generated on-line.

Surveillance systems would benefit from a generic shape recovery system for target detection and localization. Similarly, an action recognition method could improve the context of the recovered shape 
depending on the target object. For example, action recognition for human motion through a generic shape recovery system would require an a priori known model of the human, but would be able to predict the actions executed.

The entertainment field would benefit from a generic shape recovery for tasks such as motioncapture for film, virtual reality immersion, and human-robot interaction. Motion-capture techniques are popular in film and video games, but rely on engineered markers and a priori known models for correspondence. A generic method for a priori unknown objects would reduce the pre-processing overhead necessary to generate the initial models and build the motion capture suits. Virtual reality immersion with full character models would similarly benefit from a generic method, as the models will be generated online, and accommodate user-held props. Finally, the generic method could be used for human-robot interactions similar to virtual-reality immersion, wherein the human's motions are directly mapped into a deforming 3D object that may interact with virtual objects.

Past solutions have focused on separate aspects of shape recovery such as recovery of a priori known objects, or recovery of a priori unknown static objects. A priori known object recovery methods could be classified into two subgroups, namely, static camera methods, and active-camera methods. Static camera method relied on the camera saturation within the workspace to maximize the total number of viewpoints available of the object. Further, strict workspace lighting and obstacle control enabled such systems to accurately recover the shape of the a priori known objects by fitting the known model to the recovered data. Active-camera methods were able to reduce the total number of cameras in the workspace and relax lighting and obstacle constraints by reconfiguring the cameras on-line to improve view-points. The recovered data was also fitted to the a priori known model to improve the quality of the recovered shape. The clear advantage of active-camera methods is their ability to overcome both obstacle occlusion, and target object self-occlusion.

The shape recovery of a priori unknown static objects was generally handled through camera saturation methods, or active-camera reconfiguration methods. The camera saturation technique relied on maximizing the total number of view-points within a controlled, obstacle-free environment to accurately recover the shape of the static target object. These methods could generate accurate models from a single demand instant by capturing image data from all cameras simultaneously. In contrast, active-camera reconfiguration methods reduced the total number of cameras necessary for shape recovery by implementing shape-exploration techniques. These methods typically consisted of one or two cameras that would transvers the controlled workspace and sample the object's surface across many demand instants to recover the complete shape. 
Past shape recovery methods have been limited by either the necessity for the a priori known object model, a controlled workspace, large number of cameras, or lack of occlusion handling, or a combination of the former. To address these deficiencies, this dissertation proposed a novel multi-camera active-vision shape recovery system. Active-camera methods reconfigure on-line to better viewpoints allowing them overcome workspace constraints such as obstacle occlusions and object self-occlusions. Further, reconfiguration, as noted in past research, reduces the total number of cameras necessary to recover the target object's shape, thus reducing the processing load. The use of passive sensors, namely cameras, instead of active sensors such as RGB-D cameras removes, in part, environmental constraints such as lighting.

\subsubsection{Modular, Multi-Camera Active-Vision System}

This dissertation developed a modular multi-camera active-vision system for the accurate shape recovery of a priori unknown deforming objects. Before developing the system, the problem was defined and generalized through a single object attribute, namely, surface area. The objectives of shape recovery were established as accuracy and completeness, wherein accuracy dictated the surface sampling error relative to the true object's model, and completeness dictated the total surface area and volume recovered. The accuracy objective depended on the object's surface sampling, while the completeness depended on the surface area and volume recovered. Since both objects relied on different attributes of the object's geometry, a modification was made to the completeness objective to generalize the objective to a single geometrical attribute of the target object, namely, the surface. The completeness objective was generalized within an optimization framework wherein the surface area visibility is maximized. Generalization ensures the objectives are applicable to both solid objects and surface-based objects.

The generalized shape recovery objectives were then applied towards handling a priori unknown deforming objects through a multi-camera active-vision system. The true model of a priori unknown objects is unavailable to the system; thus, error feedback is not feasible during surface coordinate sampling. Therefore, the accuracy objective of shape recovery for a priori unknown objects relies solely on the recovery technique implemented. The completeness objective, as handled by a surface visibility optimization, depends on the model generation method, the deformation prediction method, and the reconfiguration method. A reconfiguration method modifies camera positions and orientations online to improve the expected surface area visibility of the recovered target shape with less cameras than static camera systems. Further, reconfiguration can overcome object self-occlusions and obstacles occlusions. The reconfiguration depends on the expected object's deformation which is governed by the deformation estimation task. To account for camera motion time, reconfiguration must be based on the expected 
deformation instead of the recovered shape. To ensure reconfiguration improves the surface area visibility, the model generation method must produce a complete shape estimate of the target object.

The problem definition for shape recovery of a priori unknown deforming objects through a multicamera active-vision system resulted in three specific system tasks, namely, model generation, deformation estimation, and reconfiguration. Each task plays an integral role within the shape recovery framework, and together, the complete system can achieve the goal of accurate recovery. The complete system was designed with modular tasks to ensure robustness through user-customization. The system workflow begins by capturing images from all available cameras and parsing the image data and camera data to the model generation task. The model generation tasks generates the maximally accurate shape for the current demand instant and parses the recovered shape and camera parameters to the deformation estimation task. Deformation estimation projects the recovered shape into the next demand instant, producing a model of the expected deformation. The deformation estimation model and camera parameters are parsed to the reconfiguration method. The reconfiguration method locates the near-optimal camera poses based on stereo surface area visibility and moves the cameras via a path planner.

The model generation method consists of a fusion technique merging triangulated surface data with a visual hull. The triangulated data is recovered from all available sets of stereo-camera pairs in the workspace. The individual stereo-camera pairs are selected on-line from all possible camera-pair combinations based on their proximity. The cameras are fully calibrated to reduce the triangulation error in comparison to partially or un-calibrated cameras. Fully calibrated cameras ensured the accuracy objective of shape recovery was indirectly satisfied due to the lack error-based feedback in the system. SIFT features were chosen for stereo-camera triangulation, but the modular framework allows for alternatives. Non-keybased features cannot be used due to poor tracking and recall stability leading to poor deformation estimation. The visual hull boundary region was automatically constructed by a specialized algorithm that minimized the initial visual hull carving space. Once carved, the visual hull was fused with the triangulated surface patches through a secondary carving operation. The fusion methodology produced a complete, solid estimation of the target object with high-accuracy surface regions recovered through triangulation, and lower-accuracy maximum surface boundary regions generated from the visual hull. The method produced a complete volumetric estimate of the object's shape that is necessary for the deformation estimation and reconfiguration tasks.

The deformation estimation method implemented an adaptive particle filtering algorithm to track and predict the deformation of the generated model. The adaptive algorithm tracked all triangulated SIFT features that were temporally corresponded in each stereo-camera pair. A tracked 3D SIFT feature consisted of two 2D SIFT features that were matched between the images of a stereo-camera pair, and matched 
between the current and previous images of the same stereo-camera pair. A constant-acceleration motion model was applied within the filtering framework. The modularity of the implementation allows for alternative application-specific motion models such as sinusoidal or exponential. The number of particles used in the filter remained fixed, but the effective particle calculation could be easily implemented to dynamically update the number of particles and reduce storage overhead. The particle-filter produced the estimated motion dynamics for each tracked SIFT feature. These dynamics were then corresponded to the visual hull points in the recovered shape to project the complete model and produce a deformation estimate for the following demand instant.

The reconfiguration method was responsible for locating the near-optimal camera positions and orientation such that the stereo surface area visibility of the expected object's deformation is maximized. Stereo surface area visibility was chosen due to the implemented model generation method, namely, the highest accuracy recovered surface patches are produced from stereo-triangulation. Therefore, the logical reconfiguration metric to maximize visibility was to maximize the stereo surface area visibility. This optimization minimized the overlapping surface area visible by separate stereo-camera pairs. Reconfiguration consisted of workspace discretization, a stereo visibility function, and a camera path planner. The workspace discretization converted the continuous workspace, as subject to camera placement limits, into a set of individual placement vectors about the center of the expected target's deformation. Each stereo-camera pair's representation was generalized to reduce the total number of positional combinations of all stereo pairs in comparison to the computation of explicit positions for each camera. This generalization reduced the total number of calculations to a sub-exponential time complexity, $\mathrm{O}\left(c^{n}\right)$. The visibility function implemented a modular design with selective visibility attributes based on camera parameters. The visibility of each generalized stereo-camera pair was evaluated for each placement vector, and the near-optimal placements were chosen such that the total stereo surface area visibility was maximized. Each stereo-pair's path was then planned using a Bézier-curve trajectory to ensure SIFT tracking stability.

Ensemble, the three modular tasks produced the complete multi-camera active-vision system for shape recovery of a priori unknown deforming objects. The proposed system was capable of accurately recovering the shape of a priori unknown deforming objects including stretching and compression deformations that are typically not handled by model-based methods. The system was also capable of reducing the total number of cameras necessary for accurate recovery compared to static camera systems that saturate the workspace with cameras. Further, through reconfiguration, the system could overcome the object's self-occlusion and obstacle occlusion when compared to static camera systems with an identical 
number of cameras. The performance of individual tasks and complete system were validated through extensive simulations and experiments.

\subsubsection{Simulation Results}

Simulations were conducted to validate the system's performance in a completely controlled environment with some real-world conditions. Blender graphical rendering software was used in conjunction with MatLab and Python scripts to facilitate an on-line, automated process. The first round of simulations tested the deformation estimation method for surface-based objects only. The second round of simulations tested the performance of the model generation technique along with the deformation estimation. The final round of simulations tested the complete system.

The deformation estimation simulations tested the accuracy of both the calibrated camera triangulation technique and the adaptive particle filtering. All cameras were modelled with infinite depths of field. Image noise was added with a uniform distribution to simulation real-world conditions. Simulations were conducted for various tracking conditions such as motion towards and away from a stereo camera pair, linear and non-linear motion, and complete loss of tracking data. The motion tests were used to evaluate the recall rate using SIFT features, and the ability of the constant-acceleration motion model to track non-linear and even sinusoidal motion. The loss of tracking data simulation tested the method's ability to recover tracking data should an occlusion occur. The method was able to automatically reacquire SIFT and continue accurately tracking. All deformation estimation simulations were normalized by the square root of the surface area of the target object based on the original shape recovery objective. The normalization removed scaling issues encountered in the simulation setup such as the size of the target object, and distance of the object relative to the cameras.

The combined model generation and deformation estimation simulations tested the shape recovery accuracy based on the predicted deformation of the target object relative to the true object's model. Simulations consisted of a solid model deforming over a set of demand instants with a varying number of fixed stereo-camera pairs. The errors calculated included Euclidean distance between projected tracked points and visual hull points normalized by the square root of the object's surface area, the normalized surface areas, and volumes. The results of the simulations were compared to another fusion method from literature to show the benefit of the methodology developed. Further, the method was compared to a system for which the target object was a priori known. The comparative results indicate improved performance of the method developed and a general trend toward the performance of an a priori known model method. Simulations were also conducted to analyze the effect of workspace camera saturation to demonstrate that increasing the number of cameras in the system tends towards decreased errors. 
The complete system simulations were quantified by the total ratio of surface area recovered relative to the true object's model for each demand instant. The complete system simulations were compared to two alternatives, static cameras, and ideal cameras. All simulations consisted of six cameras configured into three stereo pairs. The ideal simulations replaced the expected deformation model with the true object's model for the next demand instants in the system to replicate a system with a priori knowledge of the object's true deformation. All simulations were conducted with a single, dynamic workspace obstacle that would occlude one pair of the static cameras. The system's performance approached the performance of the ideal system wherein the recovered surface area proportion remained steady under certain camera motion constraints. In contrast, the static camera case experienced major losses due to both obstacle occlusion and the object's self-occlusion. It is noted that as the number of cameras increases in the workspace, the performance of the static camera would approach both the ideal case less the surface area proportion lost to obstacle occlusion. The performance of the system in comparison to static cameras is nearly identical when no obstacles are present and camera reconfiguration is superficially limited. For example, the performance difference of six static cameras positioned circumferentially on a plane about a target object is negligible compared to six reconfigurable cameras limited to radial motion about the target object on the same plane without obstacles. The advantage of the proposed system is emphasized in dynamic obstacle-laden environments.

\subsubsection{Experimental Results}

Experiments were carried out to test the complete system in real-world conditions using six Canon DSLR cameras configured into three stereo-camera pairs. Each camera was calibrated individually instead of as part of a stereo pair to conform with the system's modularity, namely, cameras could be moved to form new stereo pairs if necessary, thus rendering stereo-calibration unusable. The experiments were conducted on precision actuated linear and rotary platforms with a robotic, textured target object. All cameras, linear and rotary platforms, and the target objects were explicitly controlled through a single host PC that was responsible for running the system code.

A total of ten experiments were conducted to compare the performance of the developed system to a static camera network in the presence of an occlusion. The experiments were grouped in sets of two for a total of five different robotic deformations. An ideal experiment was not feasible as an exact model of the target robot's deformation was not available, and could not be accurately built due to the random folding of the textured skin. The major difference between the simulations and experiments was the fixed focus range on the DSLR cameras. The fixed focus limited the reconfiguration space to a fixed-radius circumference about the target object. In contrast, the simulation's workspace was a variable-radius about the target object. 
The results of the experiment were quantified by the proportion of surface area visible at each demand instant. This performance metric is related to the shape recovery completeness objective introduced in Chapter 2. All experimental runs observed a major improvement with the developed system compared to static cameras. The reconfiguration enabled the system to directly overcome obstacle occlusions and maintain a steady proportion of visible surface area. In contrast, the static cameras came under occlusions and suffered large losses in surface area visibility. The experiments validated the system as a feasible solution to the generic shape recovery problem of a priori unknown deforming objects.

\subsection{Recommendations for Future Research:}

The modular system presented in this dissertation provides a generic method for shape recovery of a priori unknown deforming objects in dynamic environments. However, as with many generic methods, improvements to the system may increase shape recovery accuracy and handle more ambiguous cases of target object shape. Several aspects of the system were targeted for future improvement and future research areas.

\subsubsection{General Improvements}

The general improvements recommended for the system include migrating from a MatLab environment to a $\mathrm{C} / \mathrm{C}++$ environment, integrating a robust segmentation process, and automating the camera focus calibration. Each improvement aims to speed up the setup time and the run-time to bring the system to realtime.

The system code was written in MatLab with various supporting functions in C++ and Python. MatLab is an excellent tool for testing algorithms and methodologies due to its robust debugging environment. The downside of MatLab is execution time that only utilizes the host PC's processor. The system code could not be complied by MatLab into an executable package due to the varying data sizes that were parsed between functions and loaded from hard drive memory. Thus, to improve the processing speed of the system, the system code should be ported into the $\mathrm{C}++$ environment and optimized. Other graphical tools such as OpenGL, OpenCV, and the Point Cloud Library could be integrated with C-based functions to increase processing speed. These graphical and computer vision APIs contain processor and GPU-optimized functions for feature detection, correspondence, image filtering and segmentation techniques. Implementing the system on both a host PC's processor and GPU would increase the overall processing speed for each demand instant.

Image segmentation was outside of the scope of this dissertation. Segmentation is based heavily on target selection for shape recovery methods. This implies that either a user must select the target object, or 
the target object is selected automatically on-line based on some pre-defined conditions. User-assisted target selection and segmentation was implemented in the system experiments while an automated target segmentation and selection method was used in simulations. Neither the experimental nor simulation segmentation and selection systems were complex. The experimental segmentation method required userbased input for each demand instant, while the simulation segmentation relied on the a priori known color of the background. Both systems could be further improved with advanced segmentation techniques available in literature. Improved segmentation techniques would allow for complete automated target segmentation in both simulations and experiments. Regardless of the segmentation method chosen, the target selection process will dictate whether the system could operate autonomously, or only automatically. Specifically, if the target selection is done without user guidance, the system could be made autonomous, while user-guided target selection may only operate automatically at best. A completely user-free target selection method could be based on motion detection. Other methods such as template matching would require either user-programmed templates, or a user-assisted machine-learning approach to train selective targets.

Another suggested improvement for real-world applications is the automatic focus calibration of DSRL cameras. An automatic focus calibration would consist of a fixed camera, and a linear motorized stage with a slanted pattern image. The camera would step through each focus step on the lens and capture an image. This process would repeat for multiple times, each one with a different location of the pattern image. The result would be a data bank of images at various focus steps and pattern distances. Each image in the bank would be processed by an edge filter to create a binary image map of all edge pixels. Each edge can then be measured for thickness wherein thinner edges represent in-focus range, and thicker edges

represent out-of-focus range. A similar approach could be applied to complete camera calibration, enabling the system to use varying focal lengths and focus distances simultaneously.

\subsubsection{On-line Model Learning}

The system developed recovered the shape at each demand instant, and predicted its deformation based on tracking data. A fixed model of the object was never recorded or kept, only the recently acquired model. One possible area of future research is an on-line model learning module that will attempt to produce a singular geometric model of the object. This module would operate similar to a priori known object shape recovery methods, namely, as the model is generated on-line, it could then be deformed and updated to fit the model recovered at the current demand instant.

The major benefit of on-line model learning is the ability to create motion capture data during the recovery process. Motion capture differs from shape recovery in that the model used in motion capture is 
constant throughout the capture process and is simply deformed by the input data. In contrast, shape recovery produces partially unique models at every demand instant. A singular model of the object would greatly benefit motion capture tasks such as animation, and would overcome the manual shape correspondence necessary for shape recovery. It should be noted that within the developed system, temporal correspondence exists in the form of point tracking, while complete model correspondence does not.

The model learning module could be expanded to estimate the object's material and internal skeletal structures through fitting algorithms. Material estimation and skeleton fitting would improve the model's robustness and could be used to further refine tracking data based on possible motion constraints such as limited stretching and compression, or limited articulated motion.

\subsubsection{Surface-based Objects}

The system developed focused on the application of solid, single manifold object. A generalized system should be applicable to both solid objects and surface-based objects. The difficulty of handling surfacebased objects is that within a generalized, autonomous system, there must be a decision-making process that decides whether the target object is a solid, or a surface. This type of decision process was outside of the scope of the work, as it mandates that the system make an informed decision without a priori data. Further, the model generation methodology may fail to create a volumetric model of the object during runtime leading to recovery errors. One possible future research area is a decision process to determine whether a target object is a single solid, multiple solids, or a surface-based object.

The system developed may not work for all surface-based objects and scenarios, but it could work under one scenario wherein the cameras' reconfiguration space is highly restricted. Such an implementation may be useful in applications such as vision-assisted surgery. Specifically, the limitation of the cameras' reconfiguration space about a surface patch of the target object will result in shape recovery that will maximize the stereo-visibility of the single surface, Figure 8.1, while over-estimating a volume behind the surface. Limiting the reconfiguration space will force the cameras to reconfigure about the surface of interest while minimizing the overlapping surface area visibility of different stereo-camera pairs. 


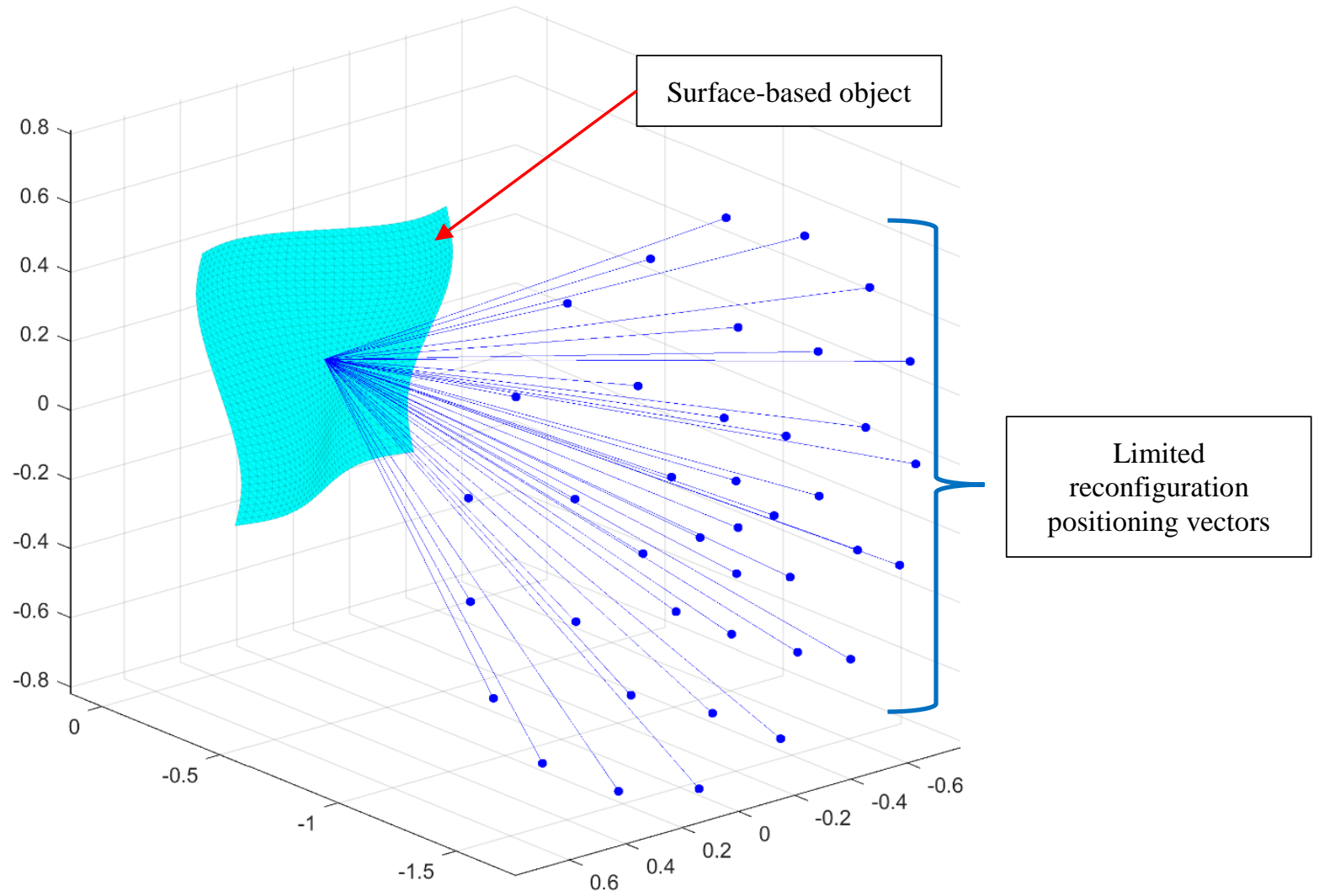

Figure 8.1: Limited reconfiguration workspace for surface-based objects. 


\section{References}

[1] D. A. Forsyth and J. Ponce, Computer vision: a modern approach, 2nd ed. Pearson, 2012.

[2] S. J. D. Prince, Computer vision: models, learning, and inference. Cambridge University Press, 2012.

[3] B. B. Le Cun, J. S. Denker, D. Henderson, R. E. Howard, W. Hubbard, and L. D. Jackel, "Handwritten digit recognition with a back-propagation network," in Advances in neural information processing systems, 1990.

[4] M. D. MacKay, R. G. Fenton, and B. Benhabib, "Pipeline-architecture based real-time activevision for human-action recognition,” J. Intell. Robot. Syst., vol. 72, no. 3-4, pp. 385-407, 2013.

[5] Y. Furukawa and J. Ponce, "Accurate, dense, and robust multiview stereopsis," IEEE Trans. Pattern Anal. Mach. Intell., vol. 32, no. 8, pp. 1362-1376, 2010.

[6] F. Blais, "Review of 20 years of range sensor development," J. Electron. Imaging, vol. 13, no. 1, pp. 231-240, 2004.

[7] P. S. Huang, C. Zhang, and F.-P. Chiang, "High-speed 3-D shape measurement based on digital fringe projection," Opt. Eng., vol. 42, no. 1, p. 163, 2003.

[8] J. F. Collado, "New methods for triangulation-based shape acquisition using laser scanners," 2004.

[9] B. Benhabib, Manufacturing: design, production, automation, and integration. CRC Press, 2003.

[10] R. J. Hocken and P. H. Pereira, Coordinate measuring machines and systems. CRC Press, 2016.

[11] T. B. Moeslund and E. Granum, "A Survey of Computer Vision-Based Human Motion Capture," Comput. Vis. Image Underst., vol. 81, no. 3, pp. 231-268, 2001.

[12] E. Sifakis, I. Neverov, and R. Fedkiw, "Automatic determination of facial muscle activations from sparse motion capture marker data," ACM Trans. Graph., vol. 24, no. 3, p. 417, Jul. 2005.

[13] G. Liu, J. Zhang, W. Wang, and L. McMillan, "Human motion estimation from a reduced marker set," in Proceedings of the 2006 symposium on Interactive 3D graphics and games - SI3D '06, 2006, p. 35.

[14] T. Antoniades, "Creating a live real-time performance-captured digital human," in $A C M$ SIGGRAPH 2016 Real-Time Live! on - SIGGRAPH '16, 2016, pp. 21-21.

[15] R. White, K. Crane, and D. a. Forsyth, "Capturing and animating occluded cloth," ACM Trans. Graph., vol. 26, no. 3, p. 34, 2007.

[16] E. de Aguiar, C. Stoll, C. Theobalt, N. Ahmed, H.-P. Seidel, and S. Thrun, "Performance Capture from Sparse Multi-View Video," ACM Trans. Graph., vol. 27, no. 3, pp. 98-108, 2008.

[17] D. Bradley, W. Heidrich, T. Popa, and A. Sheffer, "High Resolution Passive Facial Performance Capture," ACM Trans. Graph., vol. 29, no. 4, pp. 41-50, 2010.

[18] P.-L. Hsieh, C. Ma, J. Yu, and H. Li, “Unconstrained realtime facial performance capture," in 
Proceedings of the IEEE Conference on Computer Vision and Pattern Recognition, 2015, pp. 1675-1683.

[19] T. Beeler et al., "High-quality passive facial performance capture using anchor frames," ACM Trans. Graph., vol. 30, no. 4, p. 1, Jul. 2011.

[20] M. Andriluka and L. Sigal, "Human Context: Modeling Human-Human Interactions for Monocular 3D Pose Estimation," in 7th International Conference on Articulated Motion and Deformable Objects, 2012, pp. 260-272.

[21] a Sundaresan and R. Chellappa, "Acquisition of articulated human body models using multiple cameras,” Lect. Notes Comput. Sci., pp. 78-89, 2006.

[22] N. Hasler, B. Rosenhahn, T. Thormählen, M. Wand, J. Gall, and H. P. Seidel, "Markerless motion capture with unsynchronized moving cameras," in IEEE Conference on Computer Vision and Pattern Recognition (CVPR), 2009, pp. 224-231.

[23] S. Lazebnik, Y. Furukawa, and J. Ponce, "Projective visual hulls," Int. J. Comput. Vis., vol. 74, no. 2, pp. 137-165, 2007.

[24] Roshnara Nasrin P.P. and S. Jabbar, "Efficient 3D visual hull reconstruction based on marching cube algorithm," in International Conference on Innovations in Information, Embedded and Communication Systems (ICIIECS), 2015, pp. 1-6.

[25] D. Xia, D. Li, Q. Li, and S. Xu, "A Novel Approach for Computing Exact Visual Hull from Silhouettes," Opt. - Int. J. Light Electron Opt., vol. 122, no. 24, pp. 2220-2226, 2011.

[26] G. Sanmartín, J. Flores, P. Arias, J. Cudeiro, and R. Méndez, "Motion capture for clinical purposes, an approach using PrimeSense sensors," Lect. Notes Comput. Sci. (including Subser. Lect. Notes Artif. Intell. Lect. Notes Bioinformatics), vol. 7378 LNCS, pp. 273-281, 2012.

[27] S. Corazza, L. Muendermann, A. M. Chaudhari, T. Demattio, C. Cobelli, and T. P. Andriacchi, “A markerless motion capture system to study musculoskeletal biomechanics: visual hull and simulated annealing approach," Ann. Biomed. Eng., vol. 34, no. 6, pp. 1019-1029, 2006.

[28] L. Mündermann, S. Corazza, and T. P. Andriacchi, "Markerless Motion Capture for Biomechanical Applications," 2008, pp. 377-398.

[29] M. D. MacKay, R. G. Fenton, and B. Benhabib, “Time-varying-geometry object surveillance using a multi-camera active-vision system," Int. J. Smart Sens. Intell. Syst., vol. 1, no. 3, pp. 679704, 2008.

[30] D. S. Schacter, "Multi-camera active-vision system reconfiguration for deformable object motion capture," University of Toronto, 2014.

[31] J. C. P. Chan, H. Leung, J. K. T. Tang, and T. Komura, "A Virtual Reality Dance Training System Using Motion Capture Technology," IEEE Trans. Learn. Technol., vol. 4, no. 2, pp. 187-195, Apr. 2011.

[32] B. Petit et al., "Multicamera real-time 3D modeling for telepresence and remote collaboration," Int. J. Digit. Multimed. Broadcast., vol. 2010, 2010.

[33] Y. Ma, Z.-H. Mao, W. Jia, C. Li, J. Yang, and M. Sun, "Magnetic Hand Tracking for Human- 
Computer Interface,” IEEE Trans. Magn., vol. 47, no. 5, pp. 970-973, May 2011.

[34] A. Gruno, A. Liibusk, A. Ellmann, T. Oja, A. Vain, and H. Jürgenson, "Determining sea surface heights using small footprint airborne laser scanning," Proc. SPIE, vol. 8888. p. 88880R-88880R$13,2013$.

[35] P. Rieger, "Range ambiguity resolution technique applying pulse-position modulation in time-offlight scanning lidar applications," Opt. Eng., vol. 53, no. 6, p. 61614, 2014.

[36] D. Geerardyn, H. Ingelberts, R. Deleener, and M. Kuijk, "Range imaging behind semi-transparent surfaces by high-speed modulated light," Proc. SPIE, vol. 9530. p. 95300D-95300D-7, 2015.

[37] C. Altuntas, "Pair-wise automatic registration of three-dimensional laser scanning data from historical building by created two-dimensional images," Opt. Eng., vol. 53, no. 5, p. 53108, 2014.

[38] T. Terauchi, Y. Oue, and K. Fujimura, "A flexible 3D modeling system based on combining shape-from-silhouette with light-sectioning algorithm," in International Conference on 3-D Digital Imaging and Modeling, 2005, pp. 196-203.

[39] R. Fantoni et al., "High resolution laser remote imaging innovative tools for preservation of painted surfaces: information from reflectance and fluorescence data," Proc. SPIE, vol. 9065. p. 90650Z-90650Z-10, 2013.

[40] J. Zhou, L. E. Navarro-Serment, and M. Hebert, "Detection of parking spots using 2D range data," in Intelligent Transportation Systems (ITSC), 2012 15th International IEEE Conference on, 2012, pp. 1280-1287.

[41] Y. Yemez and C. J. Wetherilt, "A volumetric fusion technique for surface reconstruction from silhouettes and range data," Comput. Vis. Image Underst., vol. 105, no. 1, pp. 30-41, 2007.

[42] J. Salvi, S. Fernandez, T. Pribanic, and X. Llado, "A state of the art in structured light patterns for surface profilometry," Pattern Recognit., vol. 43, no. 8, pp. 2666-2680, 2010.

[43] B. Mobedi and G. Nejat, "An active light sensory system for 3D mapping of unknown cluttered environments," in IEEE International Conference on Automation Science and Engineering, 2010, pp. 954-959.

[44] C. Liu, S. Ma, Q. Ma, and X. Zeng, "Stereo vision system based on the laser dot matrix projection,” Proc. SPIE, vol. 8197. pp. 819704-819706, 2011.

[45] P. Fong and F. Buron, "High-Resolution Three-Dimensional Sensing of Fast Deforming Objects," in Intelligent Robots and Systems, 2005.(IROS 2005). 2005 IEEE/RSJ International Conference on, 2005, pp. 1606-1611.

[46] Y. Xu and D. G. Aliaga, "High-resolution modeling of moving and deforming objects using sparse geometric and dense photometric measurements," in Computer Vision and Pattern Recognition (CVPR), 2010 IEEE Conference on, 2010, pp. 1237-1244.

[47] G. J. Brostow, C. Hernandez, G. Vogiatzis, B. Stenger, and R. Cipolla, "Video Normals from Colored Lights," IEEE Trans. Pattern Anal. Mach. Intell., vol. 33, no. 10, pp. 2104-2114, Oct. 2011.

[48] F. Pelissier and F. Berry, "Design of a real-time embedded stereo smart camera," in Advanced 
Concepts for Intelligent Vision Systems, 2010, pp. 344-356.

[49] H. Jin, S. Soatto, and A. J. Yezzi, "Multi-view stereo reconstruction of dense shape and complex appearance," Int. J. Comput. Vis., vol. 63, no. 3, pp. 175-189, 2005.

[50] Y. Liu, Q. Dai, and W. Xu, "A point-cloud-based multiview stereo algorithm for free-viewpoint video," IEEE Trans. Vis. Comput. Graph., vol. 16, no. 3, pp. 407-418, 2010.

[51] M. Goesele, B. Curless, and S. M. Seitz, "Multi-view stereo revisited," in IEEE Computer Society Conference on Computer Vision and Pattern Recognition, 2006, vol. 2, pp. 2402-2409.

[52] M. Pollefeys, M. Vergauwen, K. Cornelis, J. Tops, F. Verbiest, and L. Van Gool, "Structure and motion from image sequences," in Proceedings of the Conference on Optical 3D Measurement Techniques, 2001, pp. 251-258.

[53] M. Lhuillier and L. Quan, "A quasi-dense approach to surface reconstruction from uncalibrated images," IEEE Trans. Pattern Anal. Mach. Intell., vol. 27, no. 3, pp. 418-433, 2005.

[54] N. Snavely, S. M. Seitz, and R. Szeliski, "Photo tourism: exploring photo collections in 3D," ACM Trans. Graph., vol. 25, no. 3, pp. 835-846, Jul. 2006.

[55] K. Li, Q. Dai, and W. Xu, "Markerless shape and motion capture from multiview video sequences," Circuits Syst. Video Technol. IEEE Trans., vol. 21, no. 3, pp. 320-334, 2011.

[56] A. Doshi, J. Starck, and A. Hilton, "An Empirical Study of Non-Rigid Surface Feature Matching of Human from 3D Video," J. Virtual Real. Broadcast., vol. 7, no. 3, pp. 1860-2037, 2010.

[57] A. Laurentini, "Visual hull concept for silhouette-based image understanding," IEEE Trans. Pattern Anal. Mach. Intell., vol. 16, no. 2, pp. 150-162, 1994.

[58] W. Matusik, C. Buehler, R. Raskar, S. J. Gortler, and L. McMillan, "Image-Based Visual Hulls," in Proceedings of the Annual Conference on Computer Graphics and Interactive Techniques, SIGGRAPH, 2000, pp. 369-374.

[59] Q. Li, S. Xu, D. Xia, and D. Li, “A novel 3D convex surface reconstruction method based on visual hull," in Pattern Recognition and Computer Vision, 2011, vol. 8004, p. 800412.

[60] Y. Furukawa and J. Ponce, "Carved visual hulls for image-based modeling," in European Conference on Computer Vision, 2006, pp. 564-577.

[61] S. Corazza, L. Mündermann, E. Gambaretto, G. Ferrigno, and T. P. Andriacchi, "Markerless motion capture through visual hull, articulated ICP and subject specific model generation," Int. J. Comput. Vis., vol. 87, no. 1-2, pp. 156-169, 2010.

[62] F. Caillette and T. Howard, "Real-time markerless human body tracking with multi-view 3-D voxel reconstruction," in Proc. BMVC, 2004, vol. 2, pp. 597-606.

[63] M. Slembrouck, J. Niño-Castañeda, G. Allebosch, D. Van Cauwelaert, P. Veelaert, and W. Philips, "High performance multi-camera tracking using shapes-from-silhouettes and occlusion removal," in Proceedings of the 9th International Conference on Distributed Smart Camera, 2015, pp. 4449.

[64] S. Tosovic, R. Sablatnig, and M. Kampe, "On Combining Shape from Silhouette and Shape from 
Structured Light," in Proc. 7th Computer Vision Winter Workshop, 2002.

[65] C. Hernández Esteban and F. Schmitt, "Silhouette and stereo fusion for 3d object modeling," Comput. Vis. Image Underst., vol. 96, no. 3, pp. 367-392, 2004.

[66] D. Cremers and K. Kolev, "Multiview stereo and silhouette consistency via convex functionals over convex domains," IEEE Trans. Pattern Anal. Mach. Intell., vol. 33, no. 6, pp. 1161-1174, 2011.

[67] P. Hebert et al., "Combined shape, appearance and silhouette for simultaneous manipulator and object tracking," in International Conference on Robotics and Automation, 2012, pp. 2405-2412.

[68] P. Song, X. Wu, and M. Y. Wang, "Volumetric stereo and silhouette fusion for image-based modeling," Vis. Comput., vol. 26, no. 12, pp. 1435-1450, Dec. 2010.

[69] Ming Li, H. Schirmacher, M. Magnor, and H.-P. Siedel, "Combining stereo and visual hull information for on-line reconstruction and rendering of dynamic scenes," in 2002 IEEE Workshop on Multimedia Signal Processing., pp. 9-12.

[70] A. Yilmaz, O. Javed, and M. Shah, "Object tracking," ACM Comput. Surv., vol. 38, no. 4, p. 13es, 2006.

[71] A. W. M. Smeulders, D. M. Chu, R. Cucchiara, S. Calderara, A. Dehghan, and M. Shah, "Visual tracking: An experimental survey," IEEE Trans. Pattern Anal. Mach. Intell., vol. 36, no. 7, pp. 1442-1468, 2014.

[72] K. Fukunaga and L. Hostetler, "The estimation of the gradient of a density function, with applications in pattern recognition," IEEE Trans. Inf. Theory, vol. 21, no. 1, pp. 32-40, Jan. 1975.

[73] Yizong Cheng, "Mean shift, mode seeking, and clustering," IEEE Trans. Pattern Anal. Mach. Intell., vol. 17, no. 8, pp. 790-799, 1995.

[74] D. Comaniciu, V. Ramesh, and P. Meer, "Real-time tracking of non-rigid objects using mean shift," in Proceedings IEEE Conference on Computer Vision and Pattern Recognition. CVPR 2000 (Cat. No.PR00662), vol. 2, pp. 142-149.

[75] D. Schugk, A. Kummert, and C. Nunn, "Adaptation of the Mean Shift Tracking Algorithm to Monochrome Vision Systems for Pedestrian Tracking Based on HoG-Features," in SAE 2014 World Congress and Exhibition, 2014, pp. 1-9.

[76] G. G. Scandaroli, M. Meilland, and R. Richa, "Improving NCC-Based Direct Visual Tracking," in European Conference on Computer Vision, 2012, pp. 442-455.

[77] N. Paragios, Y. Chen, and O. D. Faugeras, Handbook of mathematical models in computer vision. Springer Science \& Business Media, 2006.

[78] B. D. Lucas, T. Kanade, and others, “An iterative image registration technique with an application to stereo vision.," in IJCAI, 1981, vol. 81, no. 1, pp. 674-679.

[79] C. Tomasi and T. Kanade, Detection and tracking of point features. School of Computer Science, Carnegie Mellon Univ. Pittsburgh, 1991.

[80] J. Bouguet, "Pyramidal implementation of the affine lucas kanade feature tracker-description of 
the algorithm," Pages.Slc.Edu, vol. 1, no. 2, pp. 1-9, 2001.

[81] T. Wang, I. Y. H. Gu, and P. Shi, "Object Tracking using Incremental 2D-PCA Learning and ML Estimation," in 2007 IEEE International Conference on Acoustics, Speech and Signal Processing ICASSP '07, 2007, p. I-933-I-936.

[82] S. Avidan, "Support vector tracking," IEEE Trans. Pattern Anal. Mach. Intell., vol. 26, no. 8, pp. 1064-1072, Aug. 2004.

[83] D. G. Lowe, "Object Recognition from Local Scale-Invariant Features," in 7th IEEE International Conference on Computer Vision, 1999, vol. 2, pp. 1150-1157.

[84] D. G. Lowe, "Distinctive image features from scale-invariant keypoints," Int. J. Comput. Vis., vol. 60, no. 2, pp. 91-110, 2004.

[85] H. Bay, A. Ess, T. Tuytelaars, and L. Van Gool, "Speeded up robust features (SURF)," Comput. Vis. Image Underst., vol. 110, no. 3, pp. 346-359, 2008.

[86] K. Mikolajczyk and C. Schmid, "A performance evaluation of local descriptors," IEEE Trans. Pattern Anal. Mach. Intell., vol. 27, no. 10, pp. 1615-1630, Oct. 2005.

[87] G. Yu and J.-M. Morel, “ASIFT: An Algorithm for Fully Affine Invariant Comparison,” Image Process. Line, vol. 1, 2011.

[88] K. Mikolajczyk and C. Schmid, "Performance Evaluation of Local Descriptors.," IEEE Trans. Pattern Anal. Mach. Intell., vol. 27, no. 10, pp. 1615-30, 2005.

[89] B. Ristic, S. Arulampalam, and N. Gordon, "A tutorial on particle filters," in Beyond the Kalman Filter: Particle Filter for Tracking Applications, Boston: Artech House, 2004, pp. 35-62.

[90] R. E. Kalman, "A New Approach to Linear Filtering and Prediction Problems 1," ASME Trans. J. Basic Eng., vol. 82, no. Series D, pp. 35-45, 1960.

[91] S. Y. Chen, "Kalman filter for robot vision: A survey," IEEE Trans. Ind. Electron., vol. 59, no. 11, pp. 4409-4420, 2012.

[92] M. Marron et al., "Comparing a Kalman Filter and a Particle Filter in a Multiple Objects Tracking Application," in IEEE International Symposium on Intelligent Signal Processing, 2007, pp. 1-6.

[93] K. A. Joshi and D. G. Thakore, "A survey on moving object detection and tracking in video surveillance system," IJSCE, ISSN, pp. 2231-2307, 2012.

[94] S. Sedai, M. Bennamoun, and D. Q. Huynh, "A Gaussian Process Guided Particle Filter For Tracking 3D Human Pose In Video," IEEE Trans. Image Process., vol. 22, no. 11, pp. 4286-4300, 2013.

[95] W. Zheng and S. M. Bhandarkar, "Face detection and tracking using a Boosted Adaptive Particle Filter," J. Vis. Commun. Image Represent., vol. 20, no. 1, pp. 9-27, 2009.

[96] C. K. Cowan, "Model-based synthesis of sensor location," in IEEE International Conference on Robotics and Automation (ICRA), 1988, pp. 900-905.

[97] K. A. Tarabanis, P. K. Allen, and R. Y. Tsai, "A survey of sensor planning in computer vision," 
IEEE Trans. Robot. Autom., vol. 11, no. 1, pp. 86-104, 1995.

[98] R. Pito, "A solution to the next best view problem for automated surface acquisition," IEEE Trans. Pattern Anal. Mach. Intell., vol. 21, no. 10, pp. 1016-1030, 1999.

[99] C. Freundlich, P. Mordohai, and M. M. Zavlanos, "A hybrid control approach to the Next-BestView problem using stereo vision," in 2013 IEEE International Conference on Robotics and Automation, 2013, pp. 4493-4498.

[100] M. Christie, P. Olivier, and J.-M. Normand, "Camera Control in Computer Graphics," Comput. Graph. Forum, vol. 27, no. 8, pp. 2197-2218, Dec. 2008.

[101] A. Amamra, Y. Amara, R. Benaissa, and B. Merabti, "Optimal camera path planning for 3D visualisation," in SAI Computing Conference, 2016, pp. 388-393.

[102] M. Christie, R. Machap, J.-M. Normand, P. Olivier, and J. Pickering, "Virtual camera planning: a survey," in International Symposium on Smart Graphics, 2005, pp. 40-52.

[103] K. A. Tarabanis, R. Y. Tsai, and S. Abrams, "Planning viewpoints that simultaneously satisfy several feature detectability constraints for robotic vision," in International Conference on Advanced Robotics "Robots in Unstructured Environments," 1991, pp. 1410-1415 vol.2.

[104] K. A. Tarabanis, R. Y. Tsai, and P. K. Allen, "The MVP sensor planning system for robotic vision tasks," IEEE Trans. Robot. Autom., vol. 11, no. 1, pp. 72-85, 1995.

[105] S. Abrams, P. K. Allen, and K. A. Tarabanis, "Dynamic sensor planning," in IEEE International Conference on Robotics and Automation (ICRA), 1993, vol. 2, pp. 605-610.

[106] F. Schramm, F. Geffard, G. Morel, and A. Micaelli, "Calibration free image point path planning simultaneously ensuring visibility and controlling camera path," in IEEE International Conference on Robotics and Automation (ICRA), 2007, pp. 2074-2079.

[107] Z. Hermes, A. O. Nassef, and L. K. Gaafar, "Optimal Camera Path Planning for the Inspection of Printed Circuit Boards Using a Two Stepped Optimization Approach," in Volume 1: 36th Design Automation Conference, Parts A and B, 2010, pp. 745-753.

[108] N. Mir-Nasiri, "Camera-based 3D object tracking and following mobile robot," in IEEE Conference on Robotics, Automation and Mechatronics, 2006, pp. 1-6.

[109] A. Bircher, M. Kamel, K. Alexis, H. Oleynikova, and R. Siegwart, "Receding Horizon 'Next-BestView' Planner for 3D Exploration," in 2016 IEEE International Conference on Robotics and Automation (ICRA), 2016, pp. 1462-1468.

[110] P.-P. Vázquez, M. Feixas, M. Sbert, and W. Heidrich, "Automatic View Selection Using Viewpoint Entropy and its Application to Image-Based Modelling," Comput. Graph. Forum, vol. 22, no. 4, pp. 689-700, Dec. 2003.

[111] C. H. Lee, A. Varshney, and D. W. Jacobs, "Mesh saliency," in ACM SIGGRAPH 2005 Papers on - SIGGRAPH'05, 2005, p. 659.

[112] Guangfeng Ji and Han-Wei Shen, "Dynamic View Selection for Time-Varying Volumes," IEEE Trans. Vis. Comput. Graph., vol. 12, no. 5, pp. 1109-1116, Sep. 2006. 
[113] S. Takahashi, I. Fujishiro, Y. Takeshima, and T. Nishita, "A Feature-Driven Approach to Locating Optimal Viewpoints for Volume Visualization," in VIS 05. IEEE Visualization, 2005., pp. 495502.

[114] F. Benhamou, F. Goualard, É. Languénou, and M. Christie, "Interval constraint solving for camera control and motion planning," ACM Trans. Comput. Log., vol. 5, no. 4, pp. 732-767, Oct. 2004.

[115] I. Yeh, C.-H. Lin, H.-J. Chien, T.-Y. Lee, and others, "Efficient camera path planning algorithm for human motion overview," Comput. Animat. Virtual Worlds, vol. 22, no. 2-3, pp. 239-250, 2011.

[116] J. Assa, L. Wolf, and D. V. D. a C.-B. O. V. of H. M. Cohen-Or, "The virtual director: a correlation-based online viewing of human motion," Comput. Graph. Forum, vol. 29, no. 2, pp. 595-604, 2010.

[117] L. M. Wong, C. Dumont, and M. A. Abidi, "Next best view system in a 3D object modeling task," in Proceedings 1999 IEEE International Symposium on Computational Intelligence in Robotics and Automation. CIRA'99 (Cat. No.99EX375), pp. 306-311.

[118] C. Liska and R. Sablatnig, “Adaptive 3D acquisition using laser light,” in Czech Pattern Recognition Workshop, 2000, pp. 111-117.

[119] S. Y. Chen and Y. F. Li, "Vision sensor planning for 3-d model acquisition," IEEE Trans. Syst. Man, Cybern. Part B Cybern., vol. 35, no. 5, pp. 894-904, 2005.

[120] J. I. Vásquez and L. E. Sucar, "Next-Best-View Planning for 3D Object Reconstruction under Positioning Error," in MICAI'11 Proceedings of the 10th Mexican international conference on Advances in Artificial Intelligence - Volume Part I, 2011, pp. 429-442.

[121] J. I. Vasquez-Gomez, L. E. Sucar, R. Murrieta-Cid, and E. Lopez-Damian, "Volumetric Next-bestview Planning for 3D Object Reconstruction with Positioning Error," Int. J. Adv. Robot. Syst., vol. 11, no. 10, p. 1, 2014.

[122] M.-Y. Chan, W.-H. Mak, and H. Qu, "An efficient quality-based camera path planning method for volume exploration," in International Symposium on Visual Computing, 2008, pp. 12-21.

[123] K. S. Narayan, J. Sha, A. Singh, and P. Abbeel, "Range Sensor and Silhouette Fusion for HighQuality 3D Scanning," sensors, vol. 32, no. 33, p. 26, 2015.

[124] C. Piciarelli, L. Esterle, A. Khan, B. Rinner, and G. L. Foresti, "Dynamic reconfiguration in camera networks: a short survey," IEEE Trans. Circuits Syst. Video Technol., vol. 26, no. 5, pp. 965-977, May 2016.

[125] S. Indu, S. Chaudhury, N. R. Mittal, and A. Bhattacharyya, "Optimal sensor placement for surveillance of large spaces," in International Conference on Distributed Smart Cameras (ICDSC), 2009, pp. 1-8.

[126] A. Mittal and L. S. Davis, "Visibility Analysis and Sensor Planning in Dynamic Environments," in European Conference on Computer Vision, 2004, pp. 175-189.

[127] A. Mittal and L. S. Davis, "A general method for sensor planning in multi-sensor systems: Extension to random occlusion,” Int. J. Comput. Vis., vol. 76, no. 1, pp. 31-52, 2008. 
[128] B. Song, C. Ding, A. Kamal, J. A. Farrell, and A. K. Roy-Chowdhury, "Distributed camera networks,” IEEE Signal Process. Mag., vol. 28, no. 3, pp. 20-31, May 2011.

[129] F. Z. Qureshi and D. Terzopoulos, "Planning ahead for PTZ camera assignment and handoff," in 2009 Third ACM/IEEE International Conference on Distributed Smart Cameras (ICDSC), 2009, pp. 1-8.

[130] F. Z. Qureshi and D. Terzopoulos, "Surveillance in virtual reality: system design and multi-camera control," in IEEE Conference on Computer Vision and Pattern Recognition (CVPR), 2007, pp. 18.

[131] F. Z. Qureshi and D. Terzopoulos, "Proactive PTZ Camera Control," in Distributed Video Sensor Networks, London: Springer London, 2011, pp. 273-287.

[132] C. Ding, B. Song, A. Morye, J. A. Farrell, and A. K. Roy-Chowdhury, "Collaborative sensing in a distributed PTZ camera network," IEEE Trans. Image Process., vol. 21, no. 7, pp. 3282-3295, 2012.

[133] C. Ding, A. A. Morye, J. A. Farrell, and A. K. Roy-Chowdhury, "Opportunistic sensing in a distributed ptz camera network," in Distributed Smart Cameras (ICDSC), 2012 Sixth International Conference on, 2012, pp. 1-6.

[134] K. R. Konda, A. Rosani, N. Conci, and F. G. B. De Natale, "Smart camera reconfiguration in assisted home environments for elderly care," in European Conference on Computer Vision (ECCV), 2014, pp. 45-58.

[135] C. Piciarelli, C. Micheloni, and G. L. Foresti, "PTZ camera network reconfiguration," in International Conference on Distributed Smart Cameras (ICDSC), 2009, pp. 1-7.

[136] C. Piciarelli, C. Micheloni, and G. L. Foresti, "Occlusion-aware multiple camera reconfiguration," in International Conference on Distributed Smart Cameras (ICDSC), 2010, p. 88.

[137] R. T. Collins, O. Amidi, and T. Kanade, "An active camera system for acquiring multi-view video," in International Conference on Image Processing, 2002, pp. 1-4.

[138] B. Song, C. Soto, A. K. Roy-Chowdhury, and J. A. Farrell, "Decentralized camera network control using game theory," in 2008 Second ACM/IEEE International Conference on Distributed Smart Cameras, 2008, pp. 1-8.

[139] C. Soto, B. Song, and A. K. Roy-Chowdhur, "Distributed Multi-Target Tracking In A SelfConfiguring Camera Network," IEEE Conf. Comput. Vis. Pattern Recognit., 2009.

[140] A. Del Bimbo, F. Dini, G. Lisanti, and F. Pernici, "Exploiting distinctive visual landmark maps in pan-tit-zoom camera networks," Comput. Vis. Image Underst., vol. 114, no. 6, pp. 611-623, 2010.

[141] P. Natarajan, Trong Nghia Hoang, Kian Hsiang Low, M. Kankanhalli, T. N. Hoang, and K. H. Low, "Decision-theoretic coordination and control for active multi-camera surveillance in uncertain, partially observable environments," in International Conference on Distributed Smart Cameras (ICDSC), 2012, pp. 1-6.

[142] P. Natarajan, T. N. Hoang, Y. Wong, K. H. Low, and M. Kankanhalli, "Scalable DecisionTheoretic Coordination and Control for Real-time Active Multi-Camera Surveillance," in Proceedings of the International Conference on Distributed Smart Cameras - ICDSC '14, 2014, 
pp. 1-6.

[143] K. R. Konda and N. Conci, "Real-time reconfiguration of PTZ camera networks using motion field entropy and visual coverage," in Proceedings of the International Conference on Distributed Smart Cameras, 2014, p. 18.

[144] W. Starzyk and F. Z. Qureshi, "Learning proactive control strategies for PTZ cameras," in 2011 Fifth ACM/IEEE International Conference on Distributed Smart Cameras, 2011, pp. 1-6.

[145] J. R. Riehl, G. E. Collins, and J. P. Hespanha, "Cooperative Search by UAV Teams: A Model Predictive Approach using Dynamic Graphs," IEEE Trans. Aerosp. Electron. Syst., vol. 47, no. 4, pp. 2637-2656, 2011.

[146] M. Schwager, B. J. Julian, M. Angermann, and D. Rus, "Eyes in the sky: decentralized control for the deployment of robotic camera networks," Proc. IEEE, vol. 99, no. 9, pp. 1541-1561, Sep. 2011.

[147] J. L. A. Herrera and X. Chen, "Consensus algorithms in a multi-agent framework to solve PTZ camera reconfiguration in UAVs," in International Conference on Intelligent Robotics and Applications, 2012, pp. 331-340.

[148] M. D. Naish, E. A. Croft, and B. Benhabib, "Simulation-based sensing-system configuration for dynamic dispatching," in IEEE International Conference on Systems, Man and Cybernetics, 2001, vol. 5, pp. 2964-2969.

[149] M. D. Naish, E. A. Croft, and B. Benhabib, "Coordinated dispatching of proximity sensors for the surveillance of manoeuvring targets," Robot. Comput. Integr. Manuf., vol. 19, no. 3, pp. 283-299, 2003.

[150] A. Bakhtari, M. D. Naish, M. Eskandari, E. A. Croft, and B. Benhabib, "Active-vision-based multisensor surveillance-an implementation," IEEE Trans. Syst. Man, Cybern. Part C Appl. Rev., vol. 36, no. 5, pp. 668-680, 2006.

[151] A. Bakhtari and B. Benhabib, "An active vision system for multitarget surveillance in dynamic environments," IEEE Trans. Syst. Man, Cybern. Part B Cybern., vol. 37, no. 1, pp. 190-198, 2007.

[152] A. Bakhtari, M. D. MacKay, and B. Benhabib, "Active-vision for the autonomous surveillance of dynamic, multi-object environments," J. Intell. Robot. Syst., vol. 54, no. 4, pp. 567-593, 2009.

[153] J. K. Tan, S. Ishikawa, I. Yamaguchi, T. Naito, and M. Yokota, "3-D recovery of human motion by mobile stereo cameras," Artif. Life Robot., vol. 10, no. 1, pp. 64-68, Jul. 2006.

[154] A. Del Bue and L. Agapito, "Non-rigid stereo factorization," Int. J. Comput. Vis., vol. 66, no. 2, pp. 193-207, 2006.

[155] I. Yamaguchi, J. K. Tan, and S. Ishikawa, "A mobile motion capture system employing image transfer," IEEE Reg. 10 Annu. Int. Conf. Proceedings/TENCON, vol. 2007, no. 1, pp. 1-4, 2007.

[156] S. Yous, N. Ukita, and M. Kidode, “An assignment scheme to control multiple Pan/Tilt cameras for 3D video," J. Multimed., vol. 2, no. 1, pp. 10-19, 2007.

[157] M. D. MacKay, R. G. Fenton, and B. Benhabib, "Multi-camera active surveillance of an articulated human form - an implementation strategy," Comput. Vis. Image Underst., vol. 115, no. 
10, pp. 1395-1413, 2011.

[158] D. S. Schacter, M. Donnici, E. Nuger, M. D. MacKay, and B. Benhabib, "A multi-camera activevision system for deformable-object-motion capture," J. Intell. Robot. Syst., vol. 75, no. 3, pp. 413-441, 2014.

[159] W. Zhao, S. Gao, and H. Lin, "A robust hole-filling algorithm for triangular mesh," Vis. Comput., vol. 23, no. 12, pp. 987-997, Nov. 2007.

[160] A. Hilton, A. J. Stoddart, J. Illingworth, and T. Windeatt, "Reliable surface reconstruction from multiple range images," 1996, pp. 117-126.

[161] J. Davis, S. R. Marschner, M. Garr, and M. Levoy, "Filling holes in complex surfaces using volumetric diffusion," in Proceedings. First International Symposium on 3D Data Processing Visualization and Transmission, pp. 428-861.

[162] Z. Kalal, J. Matas, and K. Mikolajczyk, "Online learning of robust object detectors during unstable tracking," 2009 IEEE 12th Int. Conf. Comput. Vis. Work. ICCV Work. 2009, pp. 1417-1424, 2009.

[163] H. C. Longuet-Higgins, "A computer algorithm for reconstructing a scene from two projections," Readings Comput. Vis. Issues, Probl. Princ. Paradig. MA Fischler O. Firschein, eds, pp. 61-62, 1987.

[164] E. Mouragnon, M. Lhuillier, M. Dhome, F. Dekeyser, and P. Sayd, "Generic and real-time structure from motion using local bundle adjustment," Image Vis. Comput., vol. 27, no. 8, pp. 1178-1193, Jul. 2009.

[165] R. Szeliski and Sing Bing Kang, "Shape ambiguities in structure from motion," IEEE Trans. Pattern Anal. Mach. Intell., vol. 19, no. 5, pp. 506-512, May 1997.

[166] H. Zhou, Y. Yuan, and C. Shi, “Object Tracking using SIFT Features and Mean Shift," Comput. Vis. Image Underst., vol. 113, no. 3, pp. 345-352, 2009.

[167] M. Suárez et al., "CMOS-3D Smart Imager Architectures for Feature Detection,” Emerg. Sel. Top. Circuits Syst. IEEE J., vol. 2, no. 4, pp. 723-736, 2012.

[168] J. Fernández-Berni, R. A. Carmona-Galán, R. del Río, J. A. Leñero-Bardallo, M. Suárez-Cambre, and Á. Rodríguez-Vázquez, "Smart imaging for power-efficient extraction of Viola-Jones local descriptors," Proc. SPIE, vol. 9022. p. 902209, 2014.

[169] M. Brown and D. G. Lowe, "Automatic panoramic image stitching using invariant features," Int. J. Comput. Vis., vol. 74, no. 1, pp. 59-73, 2007.

[170] X. Du and K. K. Tan, "Vision-based approach towards lane line detection and vehicle localization,” Mach. Vis. Appl., vol. 27, no. 2, pp. 175-191, 2016.

[171] L. Moisan and B. Stival, "A probabilistic criterion to detect rigid point matches between two images and estimate the fundamental matrix," Int. J. Comput. Vis., vol. 57, no. 3, pp. 201-218, 2004.

[172] W. E. Lorensen and H. E. Cline, "Marching cubes: A high resolution 3D surface construction algorithm," in Proceedings of the 14th annual conference on Computer graphics and interactive techniques (SIGGRAPH), 1987, vol. 21, no. 4, pp. 163-169. 
[173] J. Liu, Y. Q. Chen, J. M. Maisog, and G. Luta, “A new point containment test algorithm based on preprocessing and determining triangles," Comput. Des., vol. 42, no. 12, pp. 1143-1150, Dec. 2010.

[174] S. W. Hasinoff, F. Durand, and W. T. Freeman, "Noise-optimal capture for high dynamic range photography," in IEEE Conference on Computer Vision and Pattern Recognition, 2010, pp. 553560.

[175] J. Lee, S. Lankton, and A. Tannenbaum, "Object Tracking and Target Reacquisition Based on 3-D Range Data for Moving Vehicles," IEEE Trans. Image Process., vol. 20, no. 10, pp. 2912-2924, 2011.

[176] I. Leizea, H. Álvarez, and D. Borro, "Real time non-rigid 3D surface tracking using particle filter," Comput. Vis. Image Underst., vol. 133, pp. 51-65, Apr. 2015.

[177] A. Takeuchi, S. Mita, and D. McAllester, "On-road vehicle tracking using deformable object model and particle filter with integrated likelihoods," in 2010 IEEE Intelligent Vehicles Symposium, IV 2010, June 21, 2010 - June 24, 2010, 2010, pp. 1014-1021.

[178] Y. Salih and A. S. Malik, "Comparison of stochastic filtering methods for 3D tracking," Pattern Recognit., vol. 44, no. 10-11, pp. 2711-2737, 2011.

[179] M. Owczarek, P. Baranski, and P. Strumillo, "Pedestrian tracking in video sequences: a particle filtering approach," in Federated Conference on Computer Science and Information Systems, 2015, pp. 875-881.

[180] B. Kwolek, T. Krzeszowski, A. Gagalowicz, K. Wojciechowski, and H. Josinski, "Real-time multi-view human motion tracking using particle swarm optimization with resampling," in International Conference on Articulated Motion and Deformable Objects (AMDO), 2012, pp. 92101.

[181] T. H. H. Maung, "Real-time hand tracking and gesture recognition system using neural networks," World Acad. Sci. Eng. Technol., vol. 50, pp. 466-470, 2009.

[182] G. Welch and G. Bishop, “An introduction to the Kalman filter," In Pract., vol. 7, no. 1, pp. 1-16, 2006.

[183] R. C. Eberhart, J. Kennedy, and others, “A new optimizer using particle swarm theory,” Proc. 6th Int. Symp. micro Mach. Hum. Sci., vol. 1, pp. 39-43, 1995.

[184] S. Chen, Y. Li, and N. M. Kwok, "Active vision in robotic systems: A survey of recent developments," Int. J. Rob. Res., vol. 30, no. 11, pp. 1343-1377, 2011.

[185] R. Richa, A. P. L. Bó, and P. Poignet, "Towards Robust 3D Visual Tracking for Motion Compensation in Beating Heart Surgery," Med. Image Anal., vol. 15, no. 3, pp. 302-315, 2011.

[186] R. Richa and P. Poignet, "Efficient 3D Tracking for Motion Compensation in Beating Heart Surgery.," Int. Conf. Med. Image Comput. Comput. Interv., vol. 11, no. 2, pp. 684-91, 2008.

[187] S. Katz, A. Tal, and R. Basri, "Direct visibility of point sets," ACM Trans. Graph., vol. 26, no. 3, pp. 1-12, Jul. 2007.

[188] T. Möller and B. Trumbore, "Fast, minimum storage ray-triangle intersection," J. Graph. Tools, 
vol. 2, no. 1, pp. 21-28, Jan. 1997.

[189] W. S. Kim, A. I. Ansar, R. D. Steele, and R. C. Steinke, "Performance analysis and validation of a stereo vision system," in IEEE International Conference on Systems, Man and Cybernetics, 2005, vol. 2, pp. 1409-1416.

[190] D. Gallup, J.-M. Frahm, P. Mordohai, M. Pollefeys, and C. Hill, "Variable Baseline / Resolution Stereo," in IEEE Conference on Computer Vision and Pattern Recognition, 2008, pp. 1-8.

[191] Blender Online Community, "Blender - a 3D modelling and rendering package." Blender Institute, Amsterdam, 2016.

[192] A. Vedaldi and B. Fulkerson, " $\{$ VLFeat $\}$ - An open and portable library of computer vision algorithms," in ACM International Conference on Multimedia, 2010.

[193] D. Shreiner and T. K. O. A. R. B. W. Group, OpenGL Programming Guide: The Official Guide to Learning OpenGL, Versions 3.0 and 3.1, 7th ed. Addison-Wesley Professional, 2009.

[194] J. E. Stone, D. Gohara, and G. Shi, "OpenCL: A Parallel Programming Standard for Heterogeneous Computing Systems,” Comput. Sci. Eng., vol. 12, no. 3, pp. 66-73, May 2010.

[195] R. B. Rusu and S. Cousins, "3D is here: Point Cloud Library (PCL)," in IEEE International Conference on Robotics and Automation (ICRA), 2011.

[196] J.-Y. Bouguet, “Camera calibration toolbox for MatLab,” 2004.

[197] M. Tang, L. Gorelick, O. Veksler, and Y. Boykov, "Grabcut in one cut," in Computer Vision (ICCV), 2013 IEEE International Conference on, 2013, pp. 1769-1776.

[198] Y. Furukawa and J. Ponce, "Dense 3d motion capture for human faces," in IEEE Conference on Computer Vision and Pattern Recognition, 2009, pp. 1674-1681.

[199] M. Jancosek and T. Pajdla, "Multi-view reconstruction preserving weakly-supported surfaces," in IEEE Conference on Computer Vision and Pattern Recognition, 2011, pp. 3121-3128.

[200] R. A. Newcombe et al., "KinectFusion: Real-time dense surface mapping and tracking," in Mixed and augmented reality (ISMAR), 2011 10th IEEE international symposium on, 2011, pp. 127-136.

[201] T. Whelan, M. Kaess, H. Johannsson, M. Fallon, J. J. Leonard, and J. McDonald, "Real-time large-scale dense RGB-D SLAM with volumetric fusion,” Int. J. Rob. Res., vol. 34, no. 4-5, pp. 598-626, 2014.

[202] J. Chen, D. Bautembach, and S. Izadi, "Scalable real-time volumetric surface reconstruction," Acm Tog, vol. 32, no. 4, p. 1, 2013.

[203] S. S. Wong and K. L. Chan, "3D object model reconstruction from image sequence based on photometric consistency in volume space," Pattern Anal. Appl., vol. 13, no. 4, pp. 437-450, 2010.

[204] C. Tomasi and T. Kanade, "Shape and motion from image streams: a factorization method.," Proc. Natl. Acad. Sci., vol. 90, no. 21, pp. 9795-9802, Nov. 1993.

[205] R. W. Sumner and J. Popović, "Deformation transfer for triangle meshes," in ACM Transactions on Graphics (TOG), 2004, vol. 23, no. 3, pp. 399-405. 


\section{Appendix A}

An example corresponding using orientation-locked features through NCC is presented in Figure A.1 to Figure A.5. Figure A.1 illustrates the scene image and matching template. The cross-correlation of the template is taken across the scene image to locate the peak pixel coordinate that matches the template, Figure A.2. The boundary of the template is mapped onto the scene about the peak coordinate Figure A.3. The same experiment is conducted with the feature patch rotated by $90^{\circ}$, Figure A.4. The cross-correlation map does not produce the correct result supporting the principle that orientation-locked features require identical orientation in both images, Figure A.5.

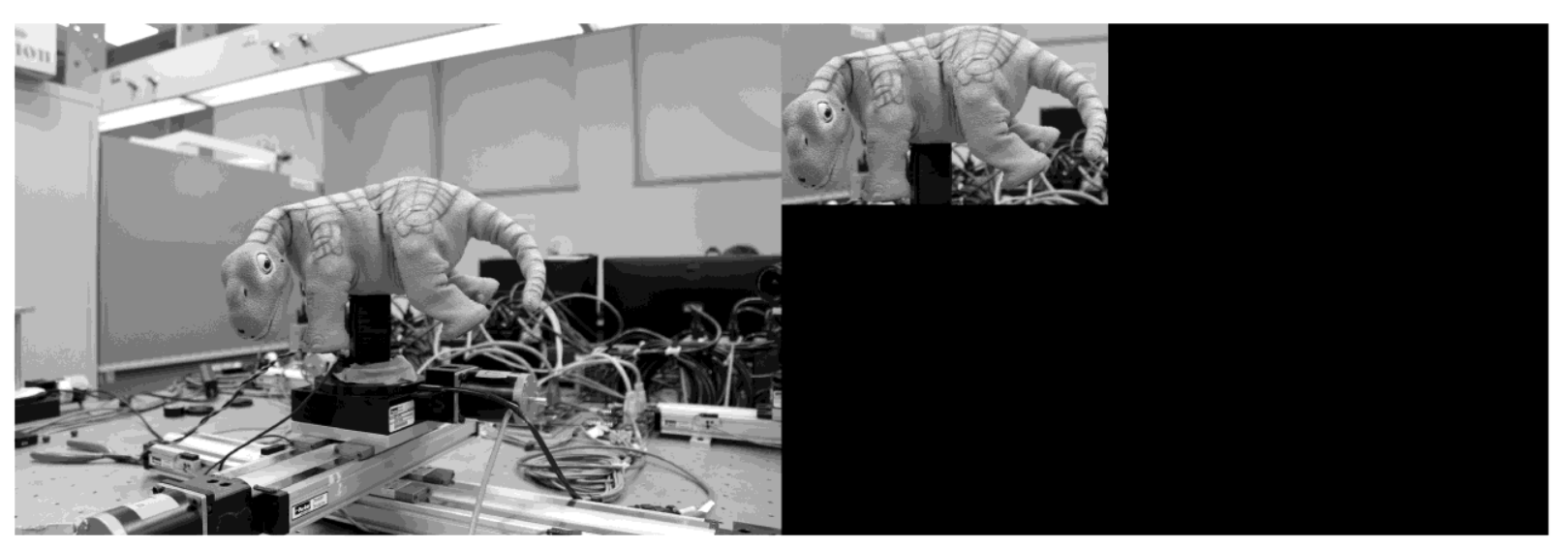

Figure A.1: Input data, left: Scene, right: matching template.

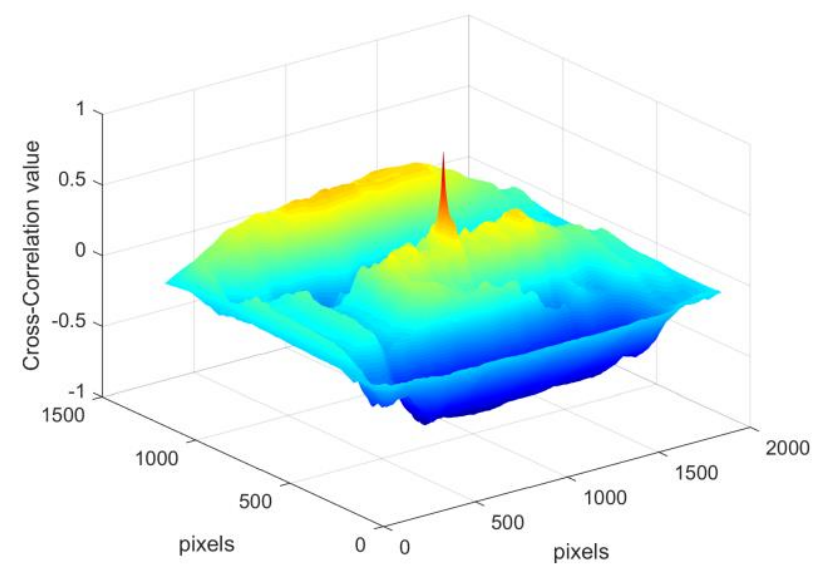

Figure A.2: Cross-correlation map. 


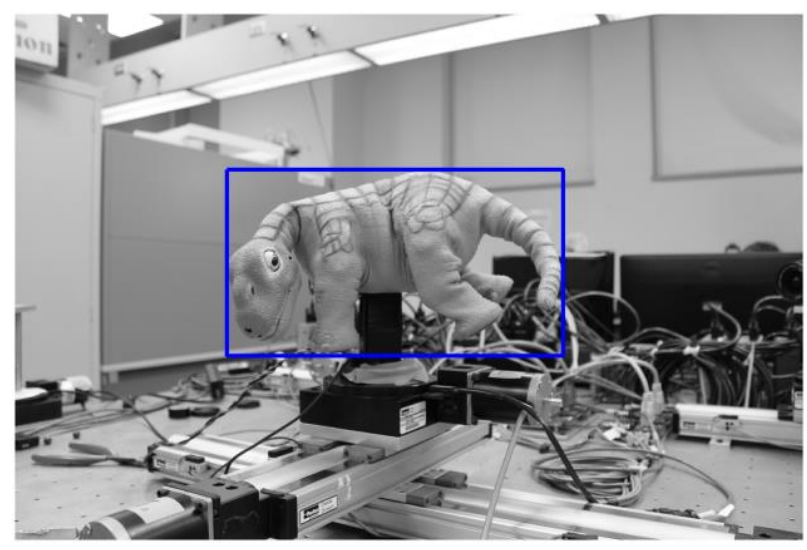

Figure A.3: Resulting cross-correlation feature match.

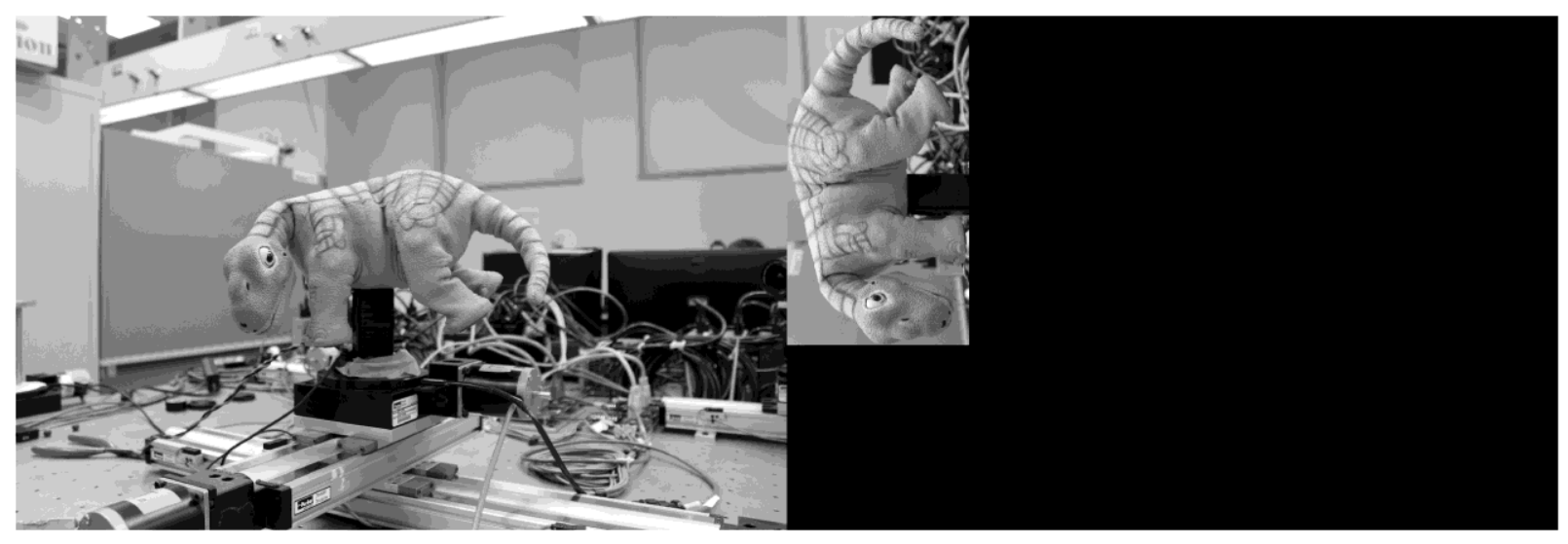

Figure A.4: Input data, left: Scene, right: matching template, rotated.

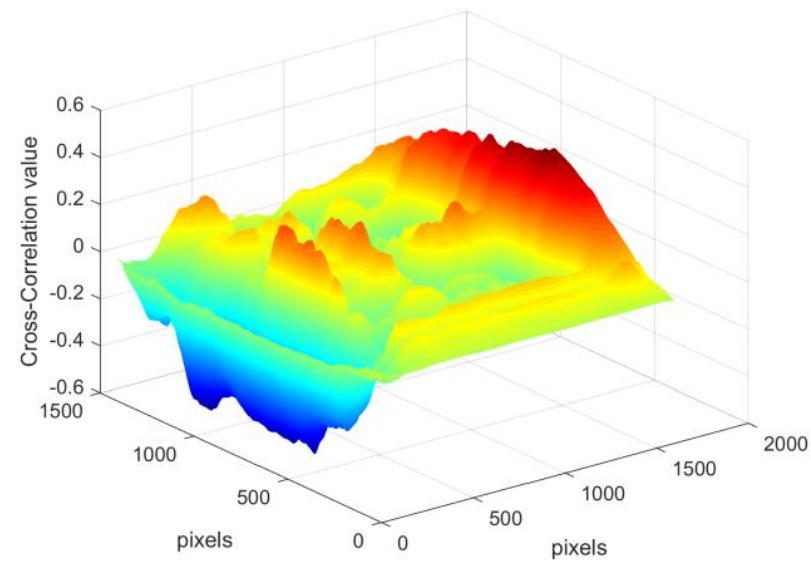

Figure A.5: Cross-correlation map. 
An example of the robustness of SIFT features is presented in Figure A.6 and Figure A.7, wherein the feature template was rotated by $90^{\circ}$ in the latter. Both matching exercises use the same scene and template as in Figure A.1 and Figure A.4. A SIFT feature detector located features in the scene image and the original template and rotated template independently and matched the feature sets. The original template produced a set of 519 matched features, while the rotated templated produced 521 matched features considering the scene image was $1296 \times 864$ pixels and the template was $553 \times 206$ pixels. The SIFT method is shown to be more robust than orientation-locked methods with a higher density of matches.

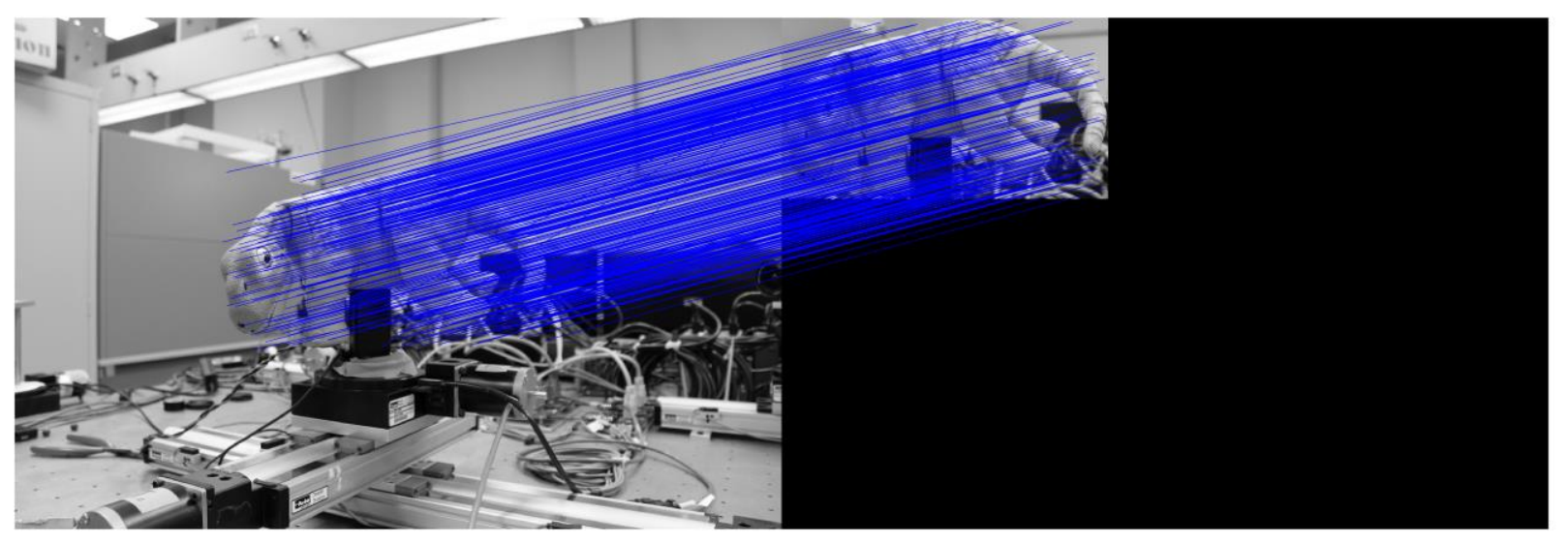

Figure A.6: SIFT matches from template to scene.

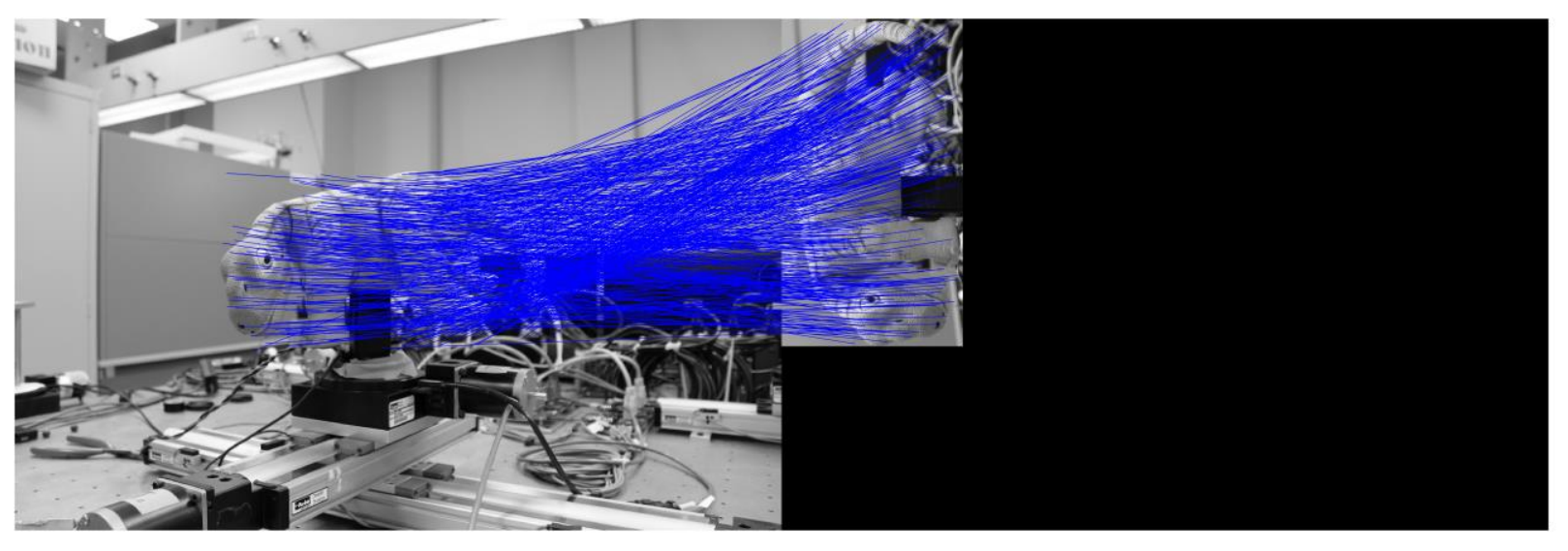

Figure A.7: SIFT matches from rotated template to scene. 


\section{Appendix B}

Supplementary figures for the simulations in Section 6.6 are presented herein.

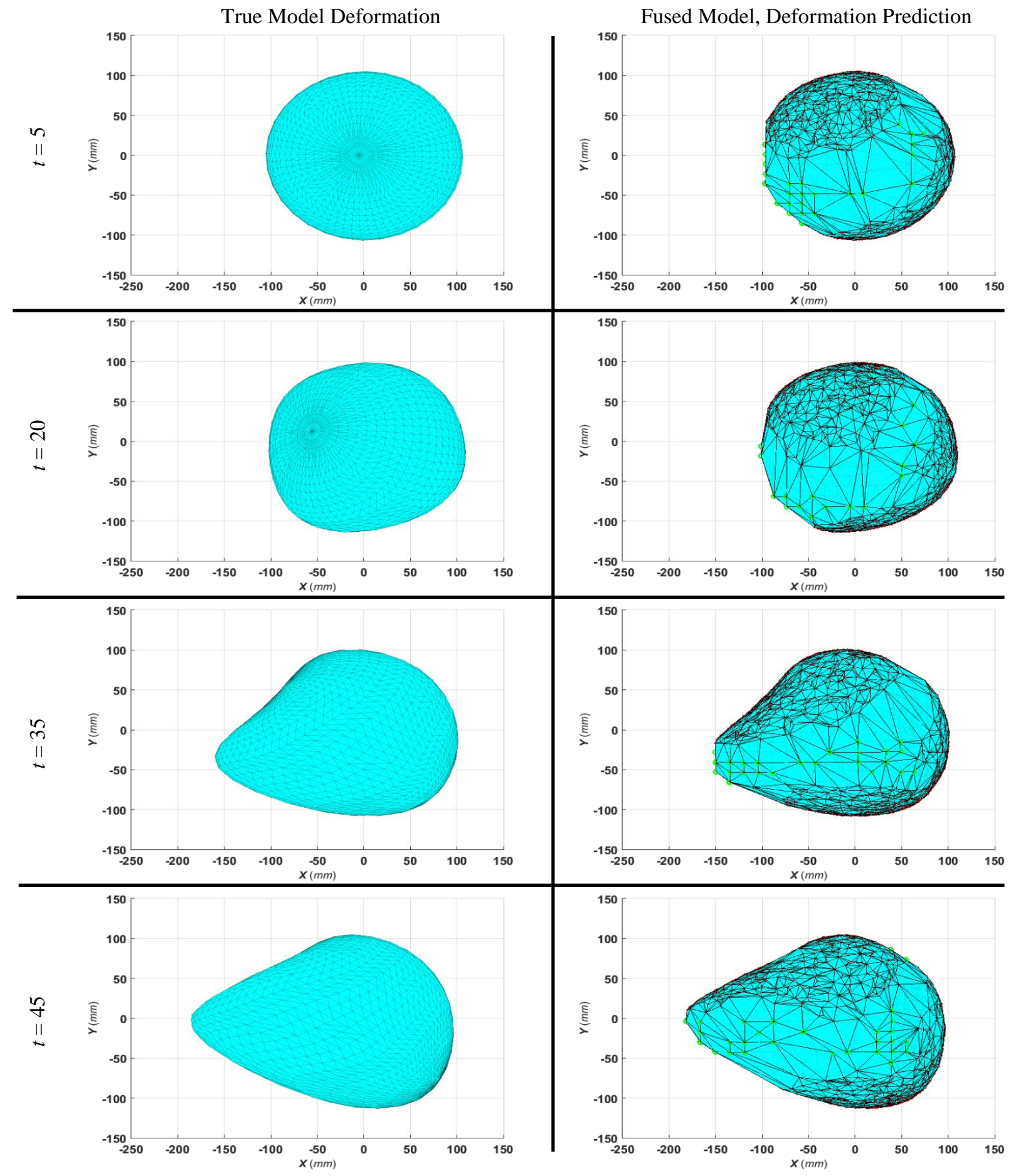

Figure B.1: Results for Simulation Sph, Alternative Viewpoint. 


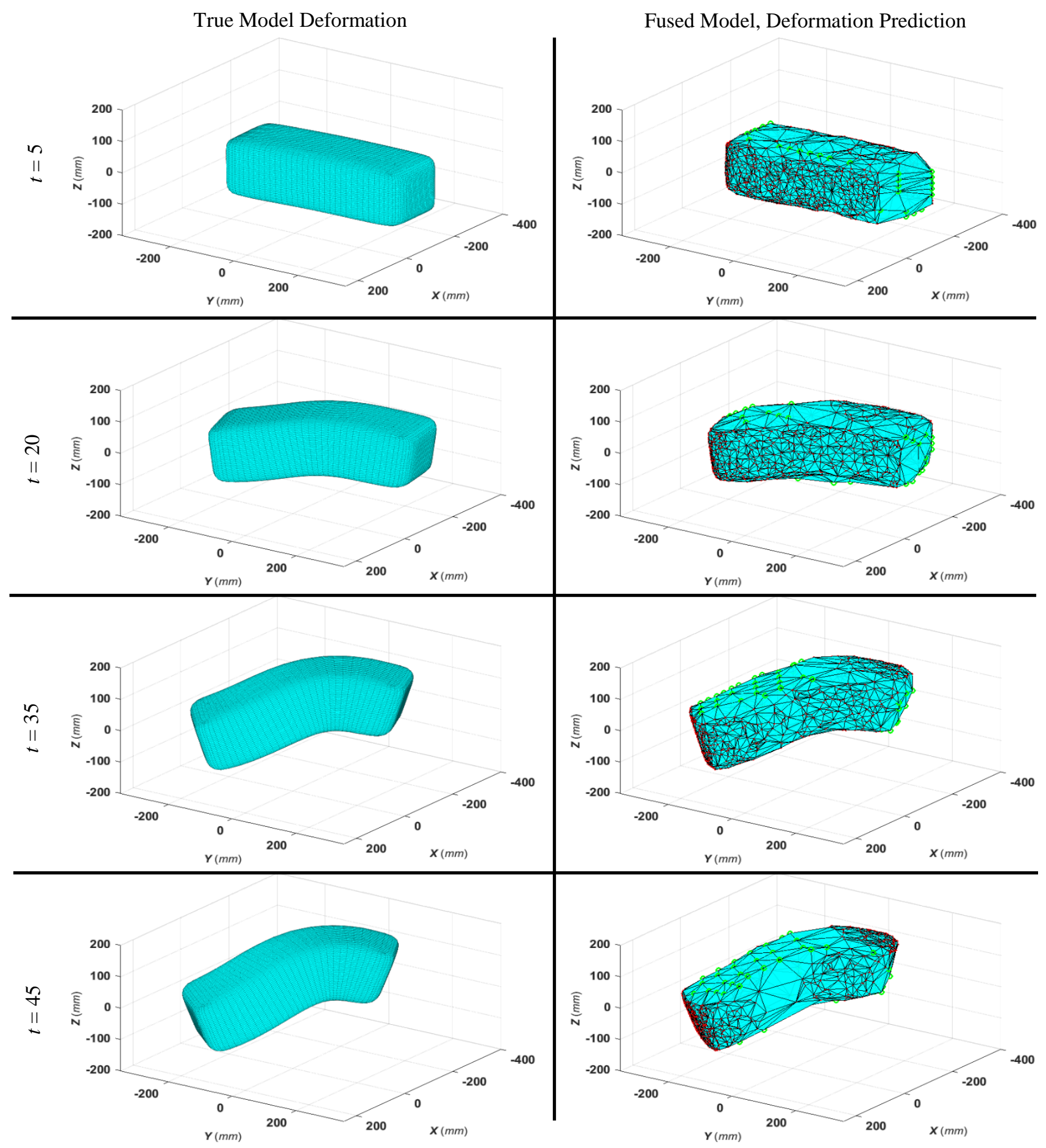

Figure B.2: Results for Simulation Prl, Alternative Viewpoint. 


\section{Appendix C}

The model generation and deformation estimation methods were shown, in Section 6.6.3, to accurately predict the deformations of two example objects with multiple camera configurations, as they deform from simple convex shapes to irregular concave shapes. In order to validate these methods, they were compared to the method developed by Li et al.[59] for a convex hull reconstruction method. The work by Li et al. implements 2D voxel cuts for improved surface resolution. Their method has been shown to recover both convex and concave geometries. It requires multiple viewpoints of the object, and a separable background. Their method, however, does not track the object, and only recovers shape at single sampling instants. Furthermore, their method does not mention how the voxel space is initialized, whereas the model generation method developed in this dissertation selects the smallest possible voxel space.

To illustrate the robustness of the model generation and deformation estimation methods, multiple simulated experiments were conducted over 50 demand instants of various deforming objects, and various camera placements. The comparative simulations are labeled as ' $S p h$ ' for the sphere, 'Prl' for the first prism deformation, and 'Pr2' for the second prism deformation, wherein 'Sph' and 'Prl' are the original simulations from Section 6.6. Simulation $S p h$ consisted of a single camera configuration identical to those in the Simulation section - Configuration $\{I I\}$. Simulation $\operatorname{Prl}$ consisted of four different camera configurations $\{I I, I V, V, V I\}$. Simulation $\operatorname{Pr} 2$ consisted of three different camera Configurations $\{I, I I, I I I\}$. The camera configurations for all simulations cases are presented in Figure B.3, Figure B.4, and Figure B.5.

The comparative results between the method presented in this paper and the method developed by Li et al. can be found in Table B.1. The results indicate the mean surface area error, mean volumetric error, and mean visual hull errors for all demand instants based on Equations (6.4) - (6.7). More importantly, the errors calculated for Li et al.'s method are for the recovered shape at each given demand instant versus the true object model, whereas the errors for the methods developed were based on the comparison of the predicted deformation of the recovered shape versus the true object model (i.e., the accuracy of the prediction made in the previous demand instant to the true model's deformation at the current demand instant).

In all the supplementary simulations, the methods developed were able to accurately estimate the shape and volume of the predicted deformation with errors comparable to Li et al.'s. The comparative results indicate that both methods are highly influenced by the camera configuration and the number of cameras. Generally, the more cameras used, the lower is the error. It is noted in Simulation Prl that the accuracy of the model for Li et al.'s method with 18 cameras approaches the accuracy of the model generation and deformation estimation methods with only 6 cameras. A particular case is highlighted in 
Simulation $\operatorname{Pr} 2$ Configurations $\{I I, I I I\}$, where the camera configuration resulted in a much higher impact on error on Li et al.'s method compared to the methods developed. This suggests that the methods developed are more robust to camera placement, specifically, poor placement. This is further supported by the comparisons in Simulation $\operatorname{Pr} 2$ Configuration $\{I\}$, where more data is recovered in the developed methods with 3 cameras, and Simulation Prl Configuration $\{I I\}$.

Table B.1: Comparative Results, Normalized Errors.

\begin{tabular}{|c|c|c|c|c|c|c|c|c|}
\hline \multirow{2}{*}{ Sim } & \multirow{2}{*}{$\begin{array}{c}\text { Camera } \\
\text { Configuration }\end{array}$} & \multirow{2}{*}{$\begin{array}{c}\text { \# of } \\
\text { Cameras }\end{array}$} & \multicolumn{2}{|c|}{$\begin{array}{l}\text { Surface Area Error } \\
\text { (\%) Eq. (6.4) }\end{array}$} & \multicolumn{2}{|c|}{$\begin{array}{l}\text { Volumetric Error } \\
(\%) \text { Eq. }(6.5)\end{array}$} & \multicolumn{2}{|c|}{$\begin{array}{l}\text { Visual Hull Error } \\
\text { (\%) Eq.(6.7) }\end{array}$} \\
\hline & & & $\begin{array}{l}\text { Li et } \\
\text { al. [59] }\end{array}$ & $\begin{array}{c}\text { Proposed } \\
\text { Method }\end{array}$ & $\begin{array}{c}\mathrm{Li} \text { et } \\
\text { al.[59] }\end{array}$ & $\begin{array}{c}\text { Proposed } \\
\text { Method }\end{array}$ & $\begin{array}{c}\mathrm{Li} \text { et } \\
\text { al.[59] }\end{array}$ & $\begin{array}{c}\text { Proposed } \\
\text { Method }\end{array}$ \\
\hline$S p h$ & $I I$ & 6 & 10.4 & 1.8 & 13.2 & 3.0 & 1.2 & 0.5 \\
\hline \multirow{4}{*}{ Prl } & $I I$ & 6 & 16.2 & 2.2 & 17.3 & 2.8 & 2.8 & 0.6 \\
\hline & $I V$ & 7 & 24.0 & 7.5 & 35.5 & 10.8 & 1.6 & 1.6 \\
\hline & V & 12 & 4.5 & 1.4 & 16.2 & 1.5 & 0.9 & 0.4 \\
\hline & $V I$ & 18 & 2.1 & 0.7 & 12.0 & 0.8 & 0.4 & 0.4 \\
\hline \multirow{3}{*}{$\operatorname{Pr} 2$} & $I$ & 3 & 15.0 & 10.2 & 19.8 & 14.1 & 1.6 & 1.5 \\
\hline & $I I$ & 6 & 26.3 & 1.6 & 32.4 & 1.5 & 5.9 & 0.6 \\
\hline & $I I I$ & 6 & 10.6 & 1.2 & 25.3 & 1.4 & 1.8 & 0.4 \\
\hline
\end{tabular}

Direct comparisons between the developed methods and methods such as dense-passive [5], [49], [51], [198], [199], dense-active [45], [46], [200]-[202], structure from motion [52]-[54], and model-based [16], [29], [30], [157], [158] could not be conducted, but discussed herein. Dense passive methods focus on maximizing the stereo-correspondence density in narrow-baseline stereo configurations. These methods rely on photoconsistent image patch matching between stereo pairs. The difference between these methods and the model generation method is that the latter implements SIFT features as the sole stereocorrespondence feature extractor which produces sparse matches, but uniquely identifiable features. The advantage of SIFT features is that the unique features could, then, be used for tracking and deformation estimation. Conversely, a densely-matched set of photoconsistent patches does create a denser surface reconstruction from triangulation, but without uniquely labelled patches and patch features, it is not possible to maintain a high recall rate on patches between a stereo camera pair and between sampling instants, as is necessary for tracking and deformation estimation. Thus, passive dense methods would produce a higher accuracy triangulated point set at a single sampling instant, but they could not be implemented for robust tracking and deformation prediction without the implementation of a methodology similar to the one presented herein. Other passive dense methods [50], [203] integrate visual hulls for recovery at single instants without active tracking. For dense-correspondence, it could also be noted that a randomly distributed texture increases the SIFT correspondences and, therefore, datasets as the ones used by Furukawa [5], [60] could not be used as the density of SIFT features was too low. 
Active recovery methods [45], [46] require pattern projection onto the target object to produce dense 3D data. The system developed within this dissertation uses passive recovery techniques, which places it into a different class of methods. The advantage of active methods is the density of triangulation, but those methods are highly susceptible to changes in lighting conditions, and do not produce unique, trackable features. The model generation method developed could operate under a larger variety of lighting conditions and recover shape passively, which may be advantageous is particular surveillance applications.

Structure from motion techniques [52]-[54] have been developed for shape estimation for uncalibrated cameras [204]. Thus, many SFM techniques benefit from large image input datasets to produce dense point clouds of target objects. Furthermore, SFM method also use key-features for correspondence between camera pairs, making them similar to the proposed method. The model generation method developed assumes that camera calibration is known and, therefore, does not need to implement a SFM technique for recovery.

Model-based shaper recovery techniques, or motion capture techniques [16], [29], [30], [157], [158] rely on a priori known skeleton or mesh model of the target object before deployment. The advantage of these methods is that shape recovery is improved under poor camera placement conditions as the known model can be accurately fit to the observed data. With a known object model, the model generation method developed could produce improved shape estimation and visibility analysis. If the model generation method developed were to assume a known target model with constant surface area and constant velocity, the errors would follow those in Table B.2. These results indicate that the known model would result in lower normalized errors, under the assumption that the model's volume and surface area are constant - which is more so the case in Simulations $\operatorname{Pr} 1$ and $\operatorname{Pr}$, while in Simulation Sph, the sphere undergoes expansion. The errors for Simulations $\operatorname{Pr} 1$ and $\operatorname{Pr} 2$ with a known model are identical for all camera configurations, as the model fitting would produce the same surface area and volume as the known initial model. Conversely, the assumption of a constant volume and surface does not work for Simulation Sph, where a known model produced larger errors due to the model's constraints. Therefore, an a priori known model could further improve the recovery, if the object's deformation minimally changes the surface area and volume. Alternatively, a robust, mesh deformation transfer [205] process would be required to fit the known model to the recovered shape under elastic deformation conditions. 
Table B.2: Comparative Results with Known Model.

\begin{tabular}{|c|c|c|c|c|c|c|}
\hline \multirow{3}{*}{ Sim } & \multirow{2}{*}{$\begin{array}{c}\text { Camera } \\
\text { Configuration }\end{array}$} & \multirow{2}{*}{$\begin{array}{c}\text { \# of } \\
\text { Cameras }\end{array}$} & \multicolumn{2}{|c|}{$\begin{array}{c}\text { Surface Area Error (\%) Eq. } \\
\mathbf{( 6 . 4 )}\end{array}$} & \multicolumn{2}{c|}{$\begin{array}{c}\text { Volumetric Error (\%) Eq. } \\
\text { (6.5) }\end{array}$} \\
\cline { 4 - 7 } & & Model Free & Model Known & Model Free & Model Known \\
\hline \multirow{3}{*}{$S p h$} & $I I$ & 6 & 1.8 & 12.1 & 3.0 & 14.7 \\
\hline \multirow{3}{*}{$\operatorname{Pr} 1$} & $I I$ & 6 & 2.2 & 0.5 & 2.6 & 0.1 \\
\cline { 2 - 7 } & $I V$ & 7 & 7.5 & 0.5 & 10.8 & 0.1 \\
\cline { 2 - 7 } & $V$ & 12 & 1.4 & 0.5 & 1.5 & 0.1 \\
\hline \multirow{3}{*}{$\operatorname{Pr} 2$} & $I I$ & 18 & 0.7 & 0.5 & 0.8 & 0.1 \\
\cline { 2 - 7 } & $I$ & 3 & 10.2 & 0.1 & 14.1 & 0.1 \\
\cline { 2 - 7 } & $I I$ & 6 & 1.6 & 0.1 & 1.5 & 0.1 \\
\hline
\end{tabular}

The following figures illustrate the camera configurations used for the simulations.
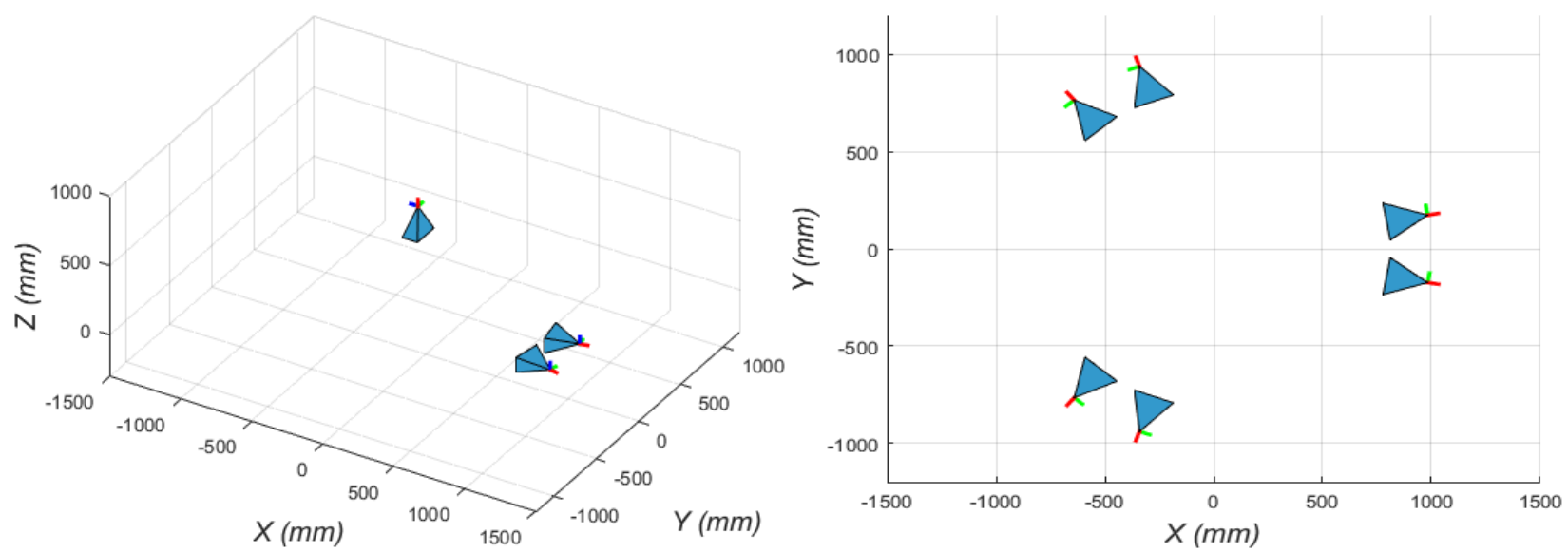

Figure B.3: Camera Configuration I (left), II (right).
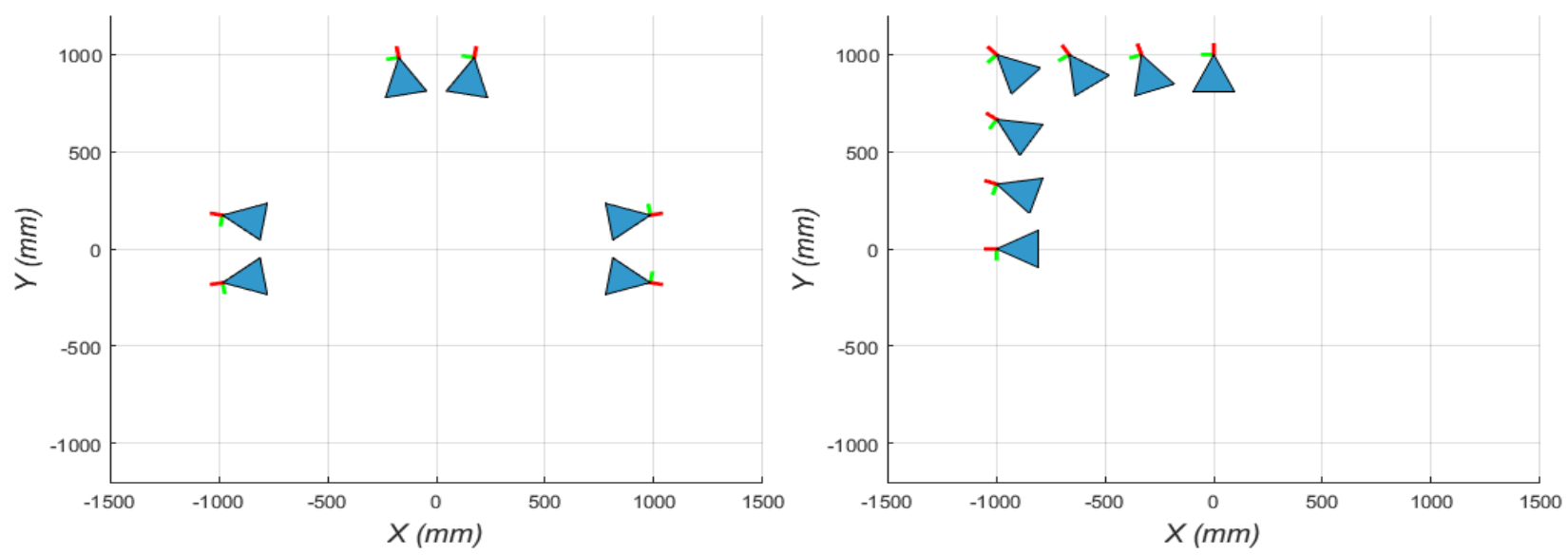

Figure B.4: Camera Configuration III (left), IV (right). 

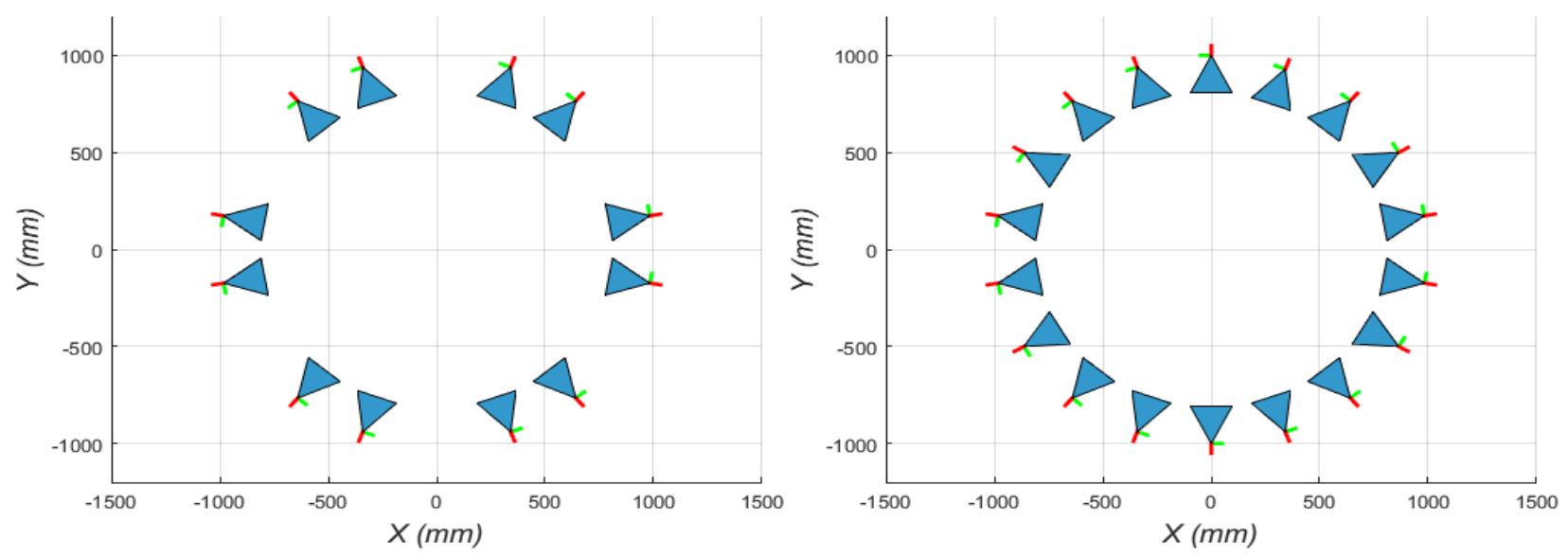

Figure B.5: Camera Configuration V (left), VI (right).

The initial and final deformations of the object for simulations 'Sph', 'Prl' and 'Pr2' are presented in Figure B.6, Figure B.7, and Figure B.8, respectively.
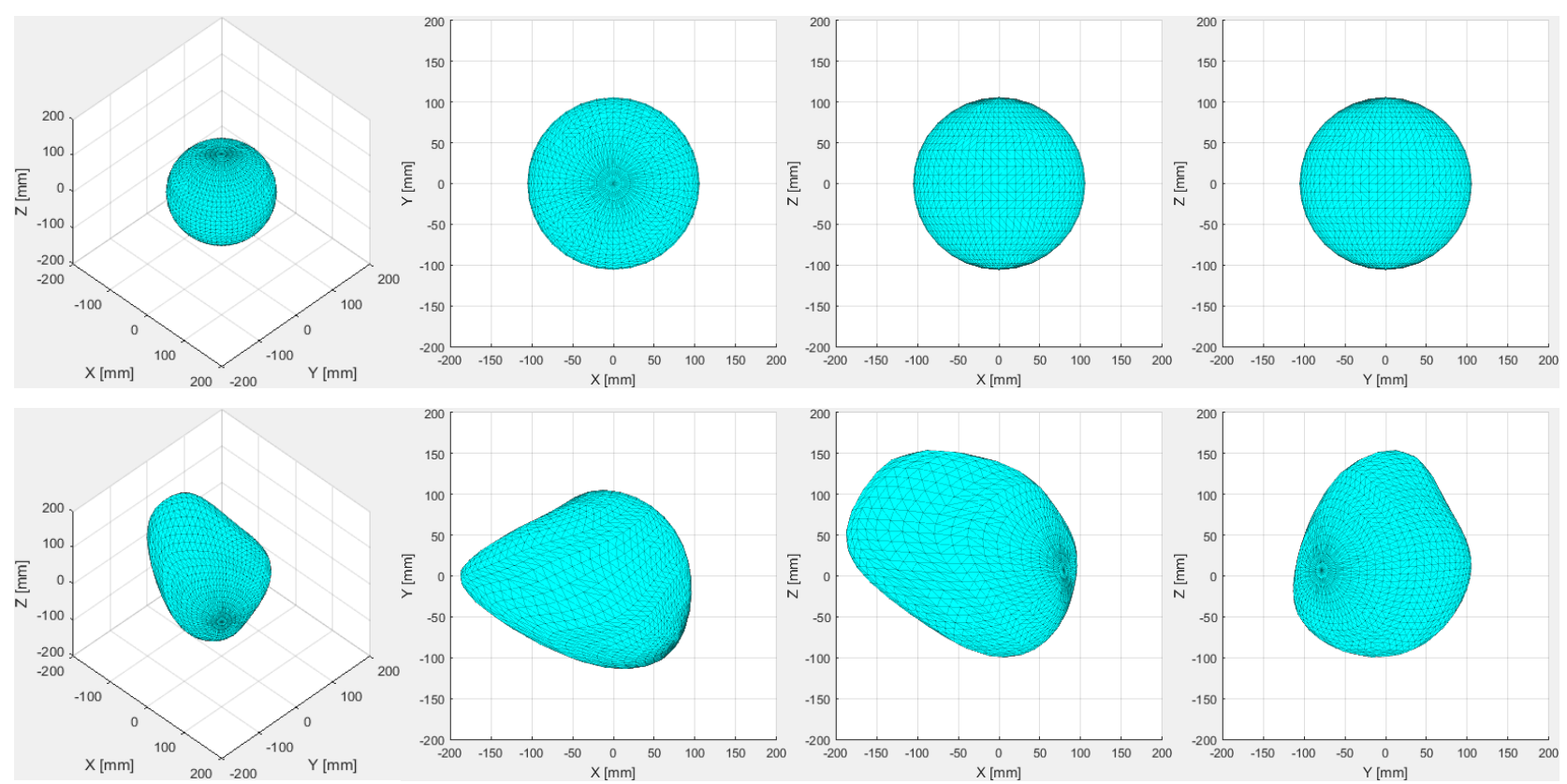

Figure B.6: Simulation Sph, Top Row - Initial Object Deformation, Bottom Row - Final Object Deformation. 

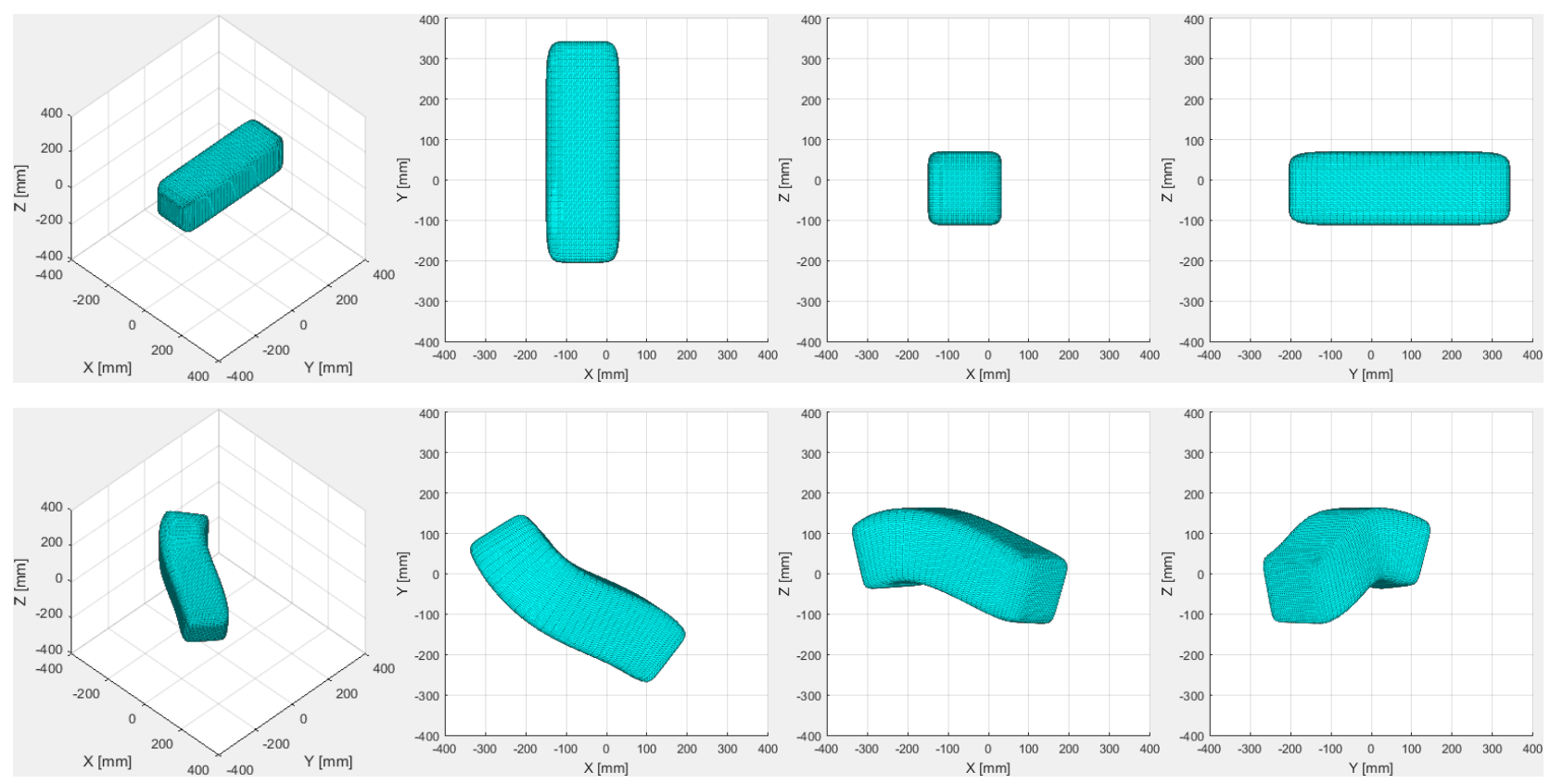

Figure B.7: Simulation Prl, Top Row - Initial Object Deformation, Bottom Row - Final Object Deformation.
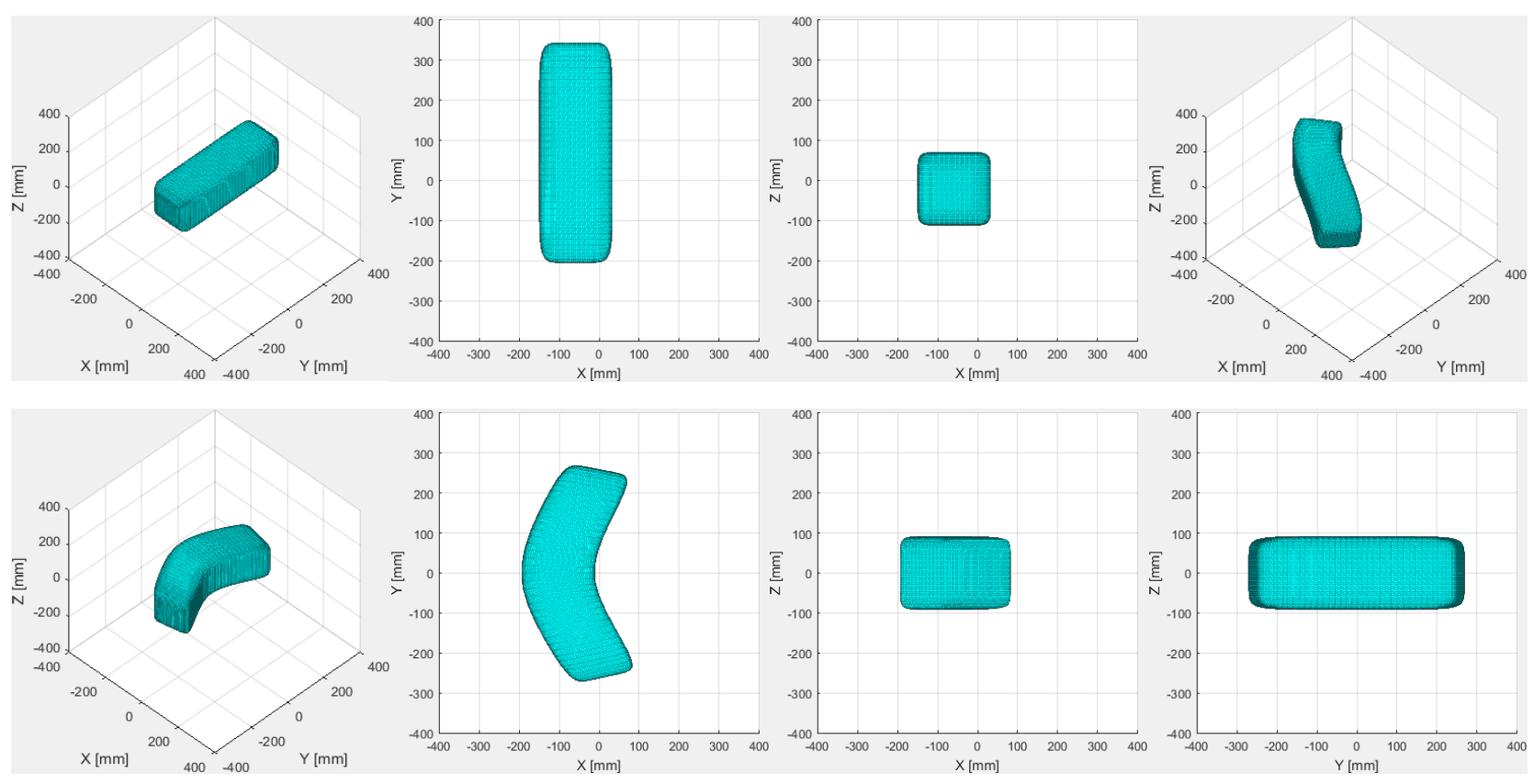

Figure B.8: Simulation Pr2, Top Row - Initial Object Deformation, Bottom Row - Final Object Deformation. 


\section{Appendix D}

The following figures illustrate the (bird-eye-view) deformation sequences and subsequent camera reconfigurations at select demand instants for the simulations described in Section 6.7. Only the estimated visible polygons of the object model are colored to illustrate the visible stereo surface area.

Figure D.1 and Figure D.2 illustrate every odd frame of the first simulation set for the static camera placement.

Figure D.3 and Figure D.4 illustrate every odd frame of the first simulation set for the reconfiguration camera placement through the proposed method.

Figure D.5 and Figure D.6 illustrate every odd frame of the first simulation set for the ideal case. 

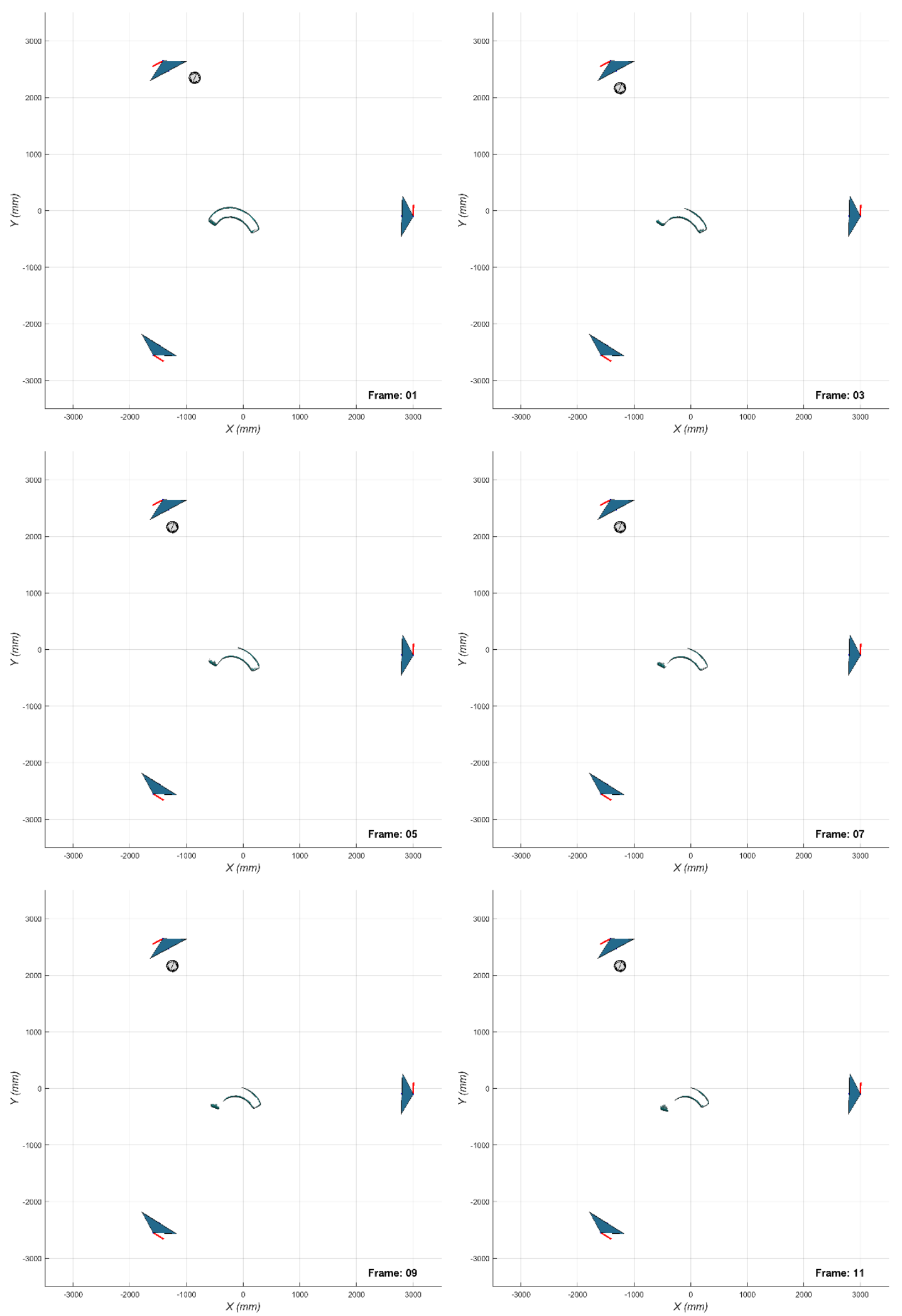

Figure D.1: Static camera Simulation Frames $\{1,3,5,7,9,11\}$, respectively. 

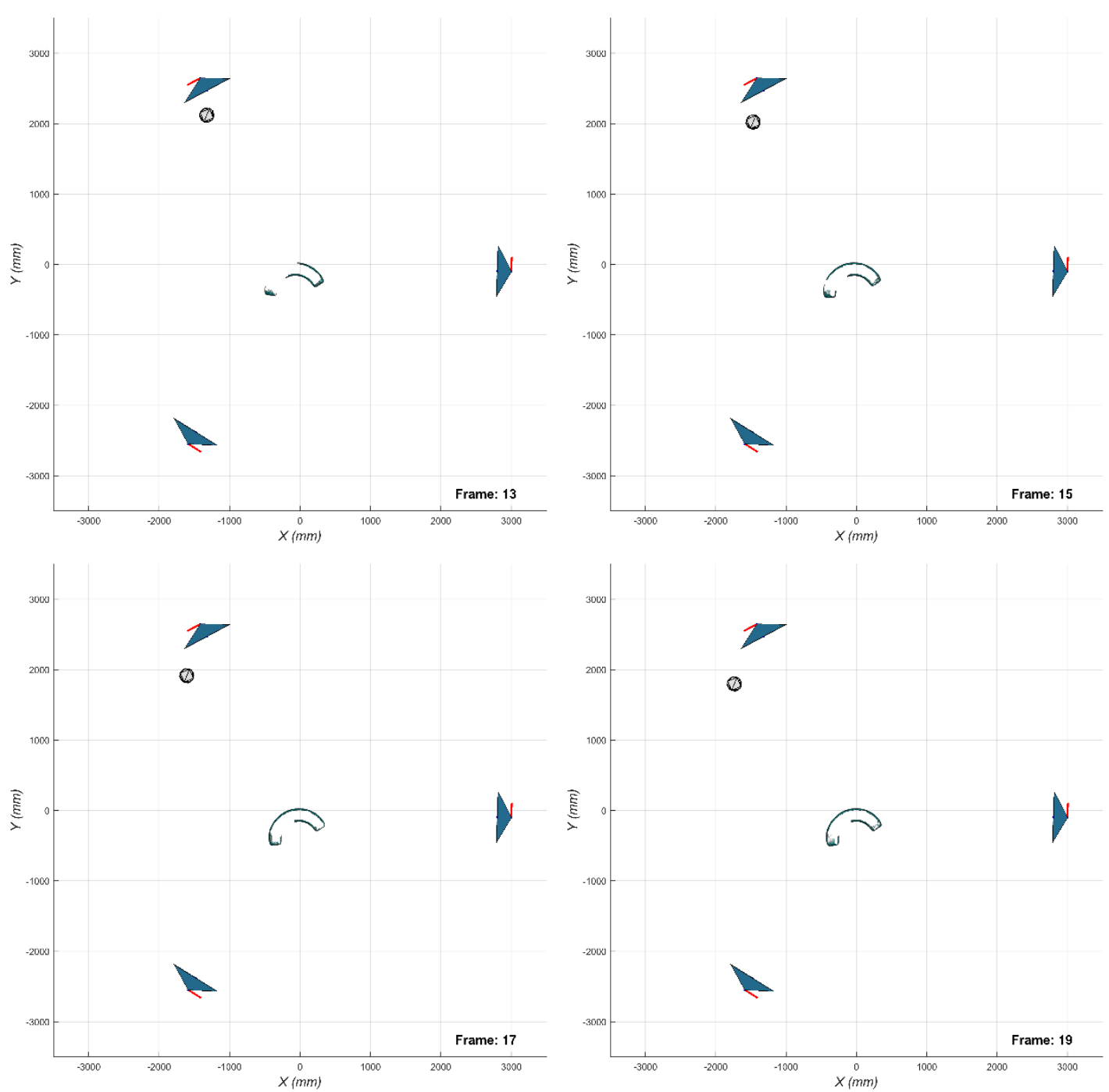

Figure D.2: Static camera Simulation Frames $\{13,15,17,19\}$, respectively. 

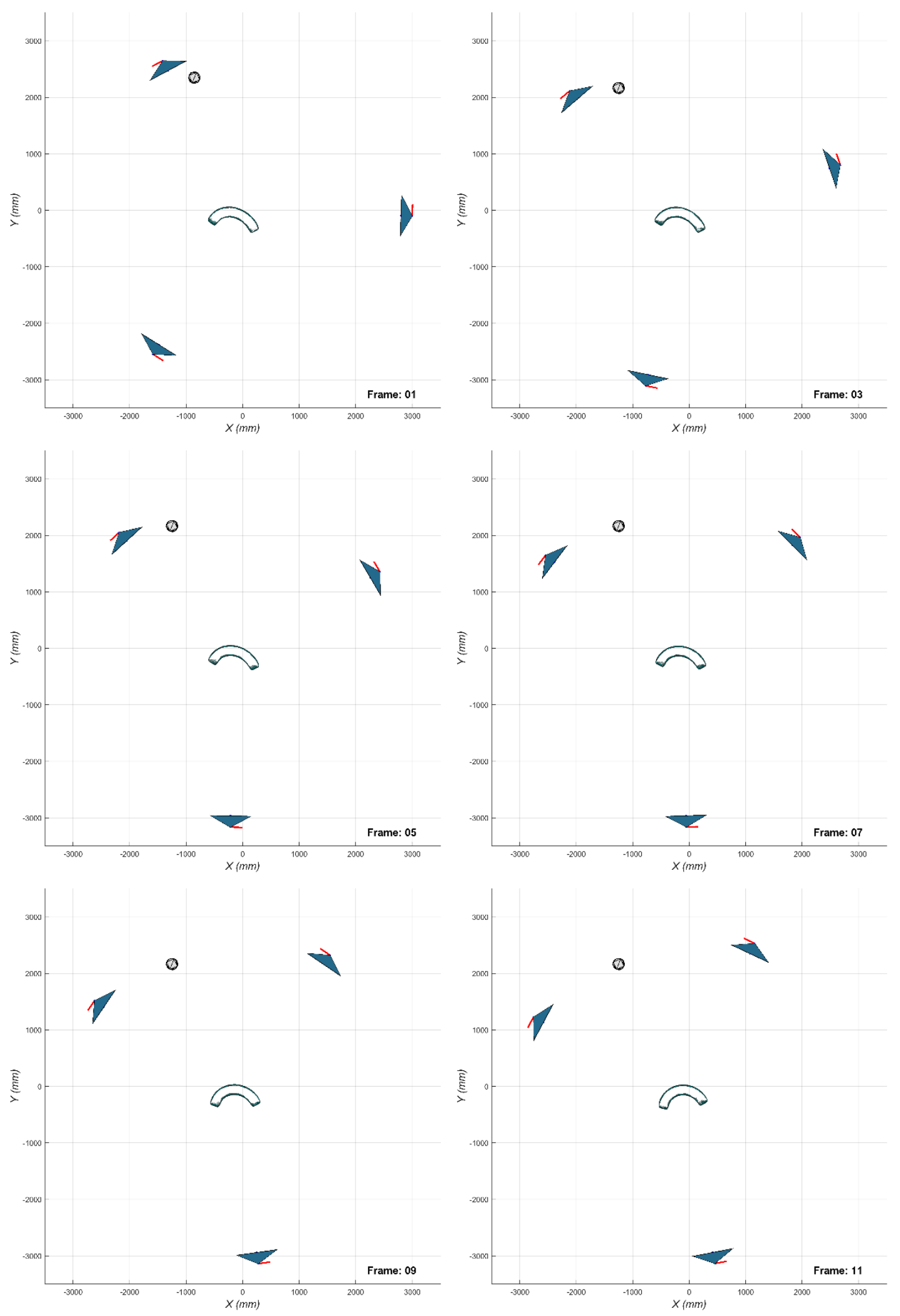

Figure D.3: Reconfiguration through proposed method, Simulation Frames $\{1,3,5,7,9,11\}$, respectively. 

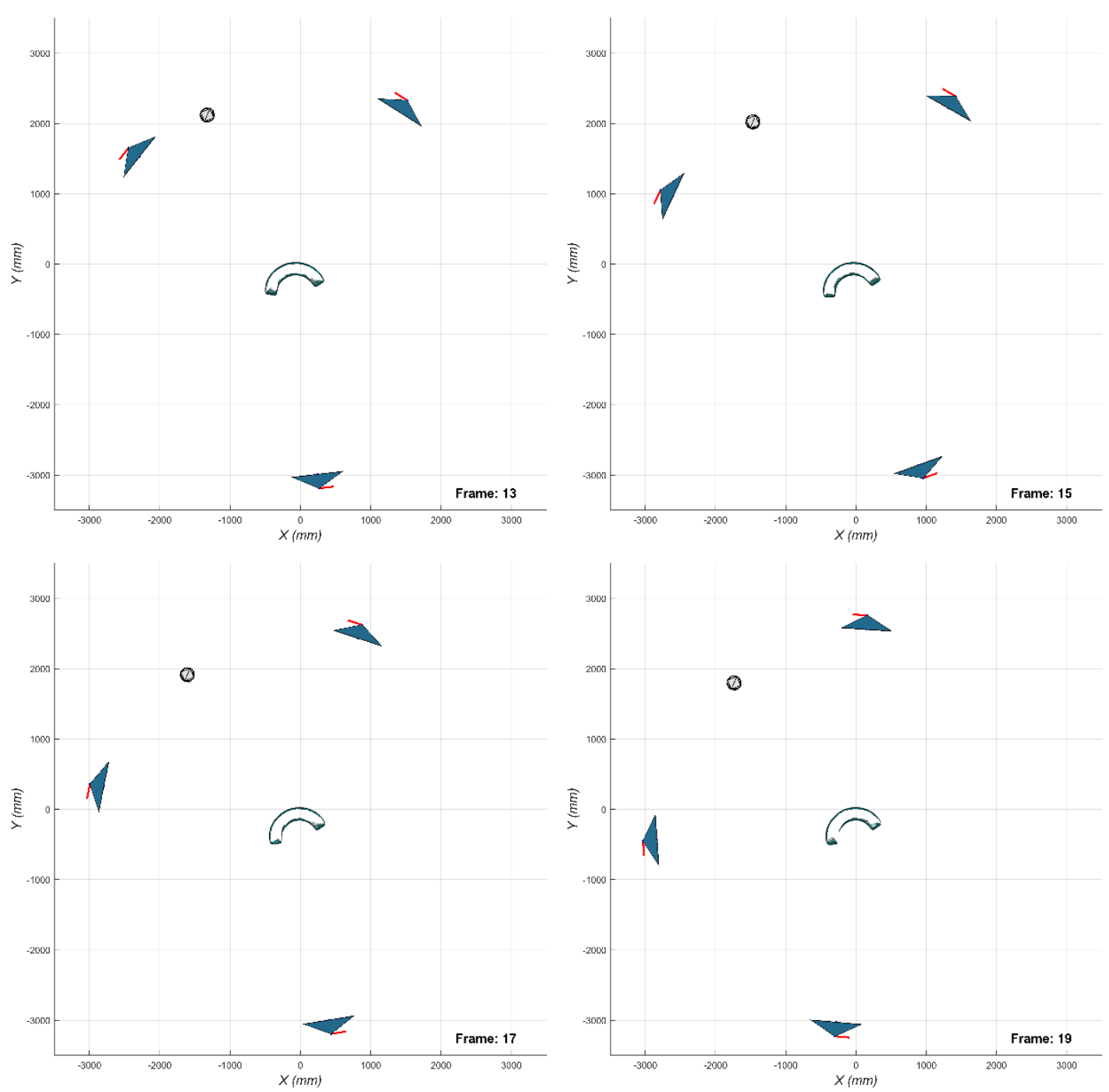

Figure D.4: Reconfiguration through proposed method, Simulation Frames $\{13,15,17,19\}$, respectively. 

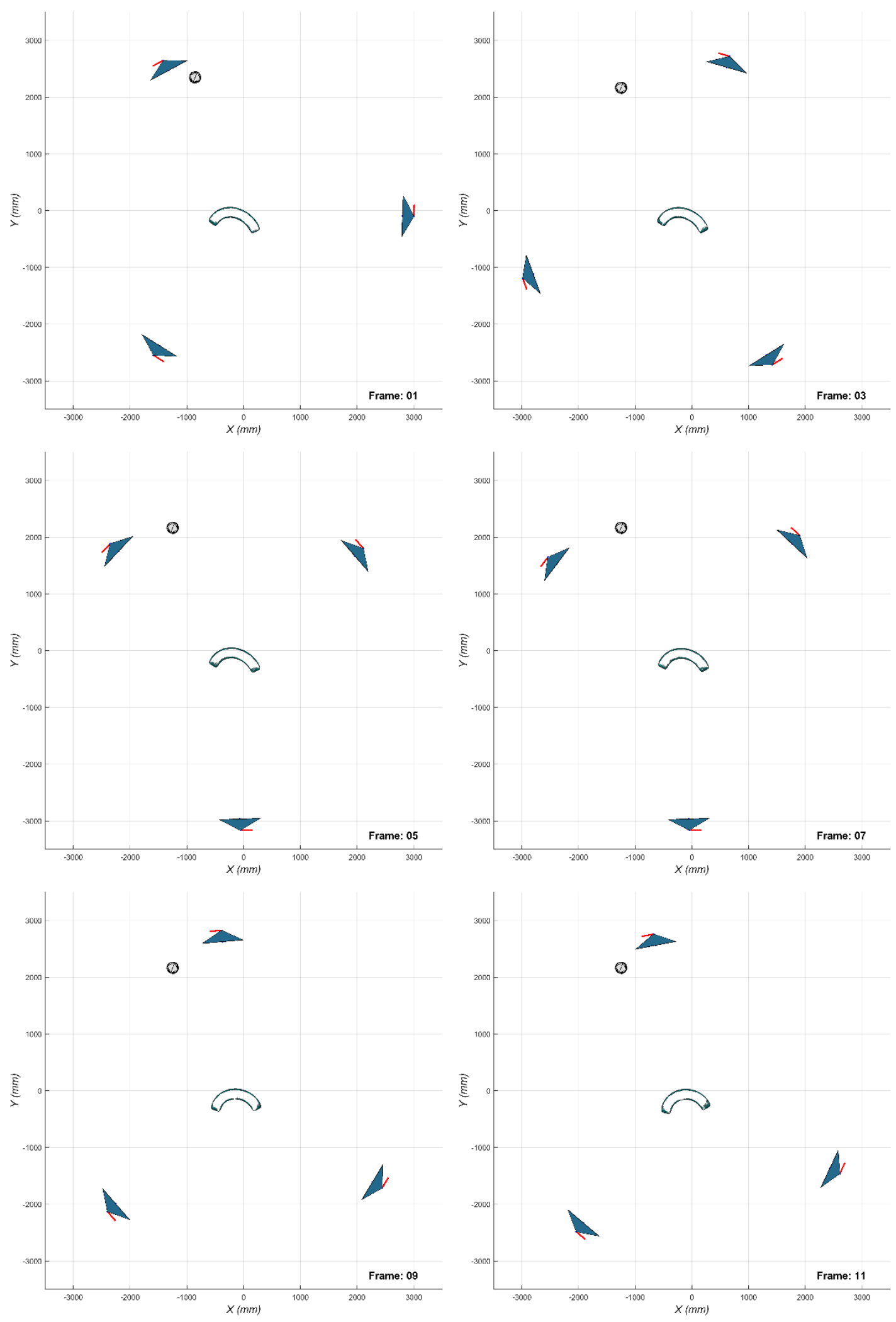

Figure D.5: Ideal camera placement, Simulation Frames $\{1,3,5,7,9,11\}$, respectively. 

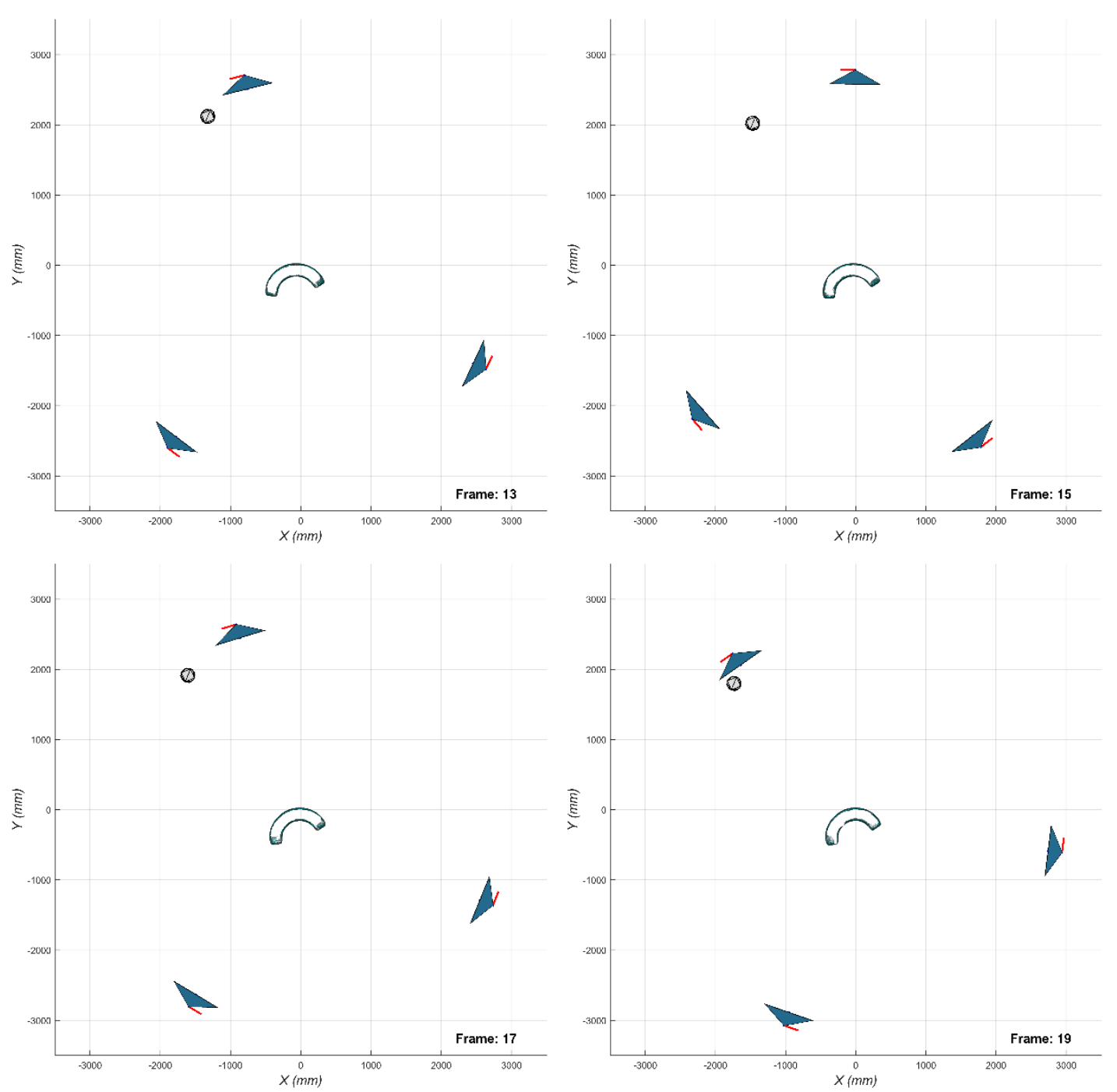

Figure D.6: Ideal camera placement, Simulation Frames $\{13,15,17,19\}$, respectively. 


\section{Appendix E}

This appendix illustrates the surface texture testing for the PLEOrb robot with the VLFeat SIFT feature detection and matching library. A close-up view of the texture and skin color of the PLEOrb robot is presented in Figure E.1. The robot has a rough surface texture with bumps along with a varying green shaded color. The texture should result in dense SIFT feature detection for high resolution images. The Canon DSRL cameras were configured into an arbitrary stereo pair, and both captured synchronized images of the robot on the camera calibration workstation, Figure E.2.

The VLFeat SIFT detection and matching algorithm was used to detect the SIFT features and match them in each image. The 2D and 3D filtering processes that were described in Sections 3.4.3 and 3.4.4 where applied with incomplete background segmentation for evaluation purposes. The incomplete segmentation resulted in several features matched outside of the robot. The matched 2D features are presented in Figure E.3. The triangulated point cloud from the PLEOrb robot is presented in isometric view, top view and front view in Figures E.4 - E.6, respectively. The results of the evaluation indicate that the surface color and texture of the PLEOrb robot produce a dense SIFT feature point cloud that corresponds well within stereo-camera pairs, and produces a dense 3D triangulated point cloud.

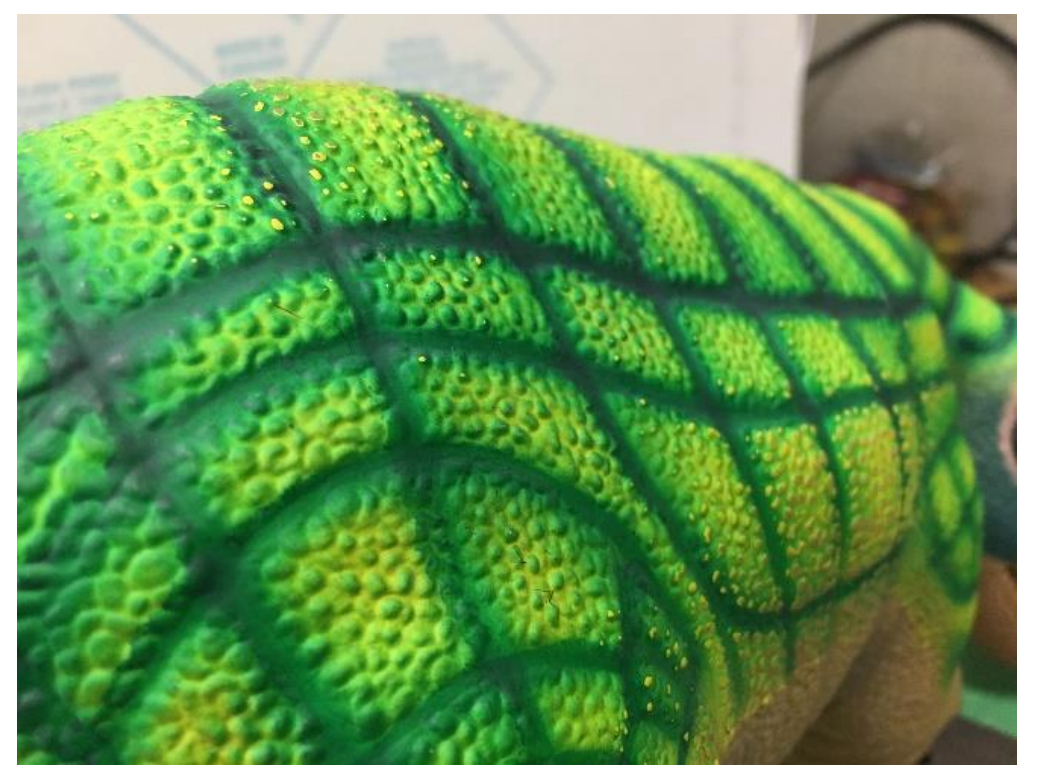

Figure E.1: Close-up view of the PLEOrb robot skin texture and color pattern. 


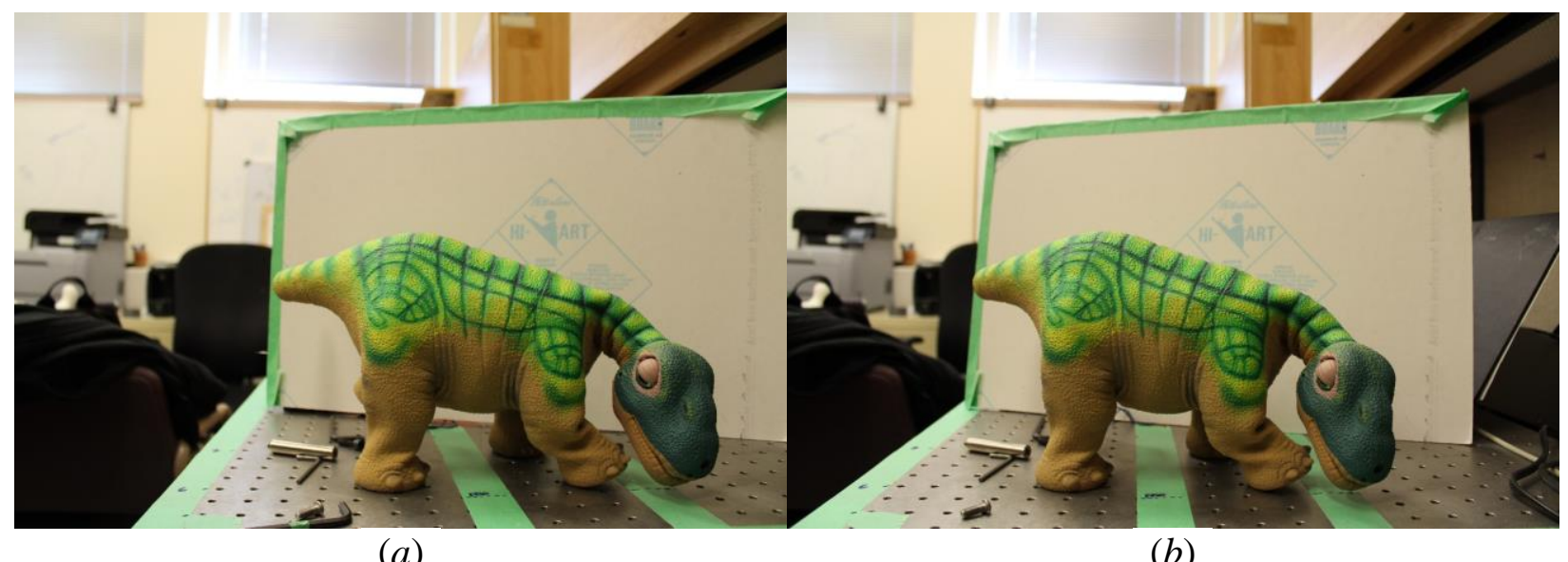

Figure E.2: (a) View from left camera in arbitrary stereo pair, $(b)$ view from right camera of arbitrary stereo pair.

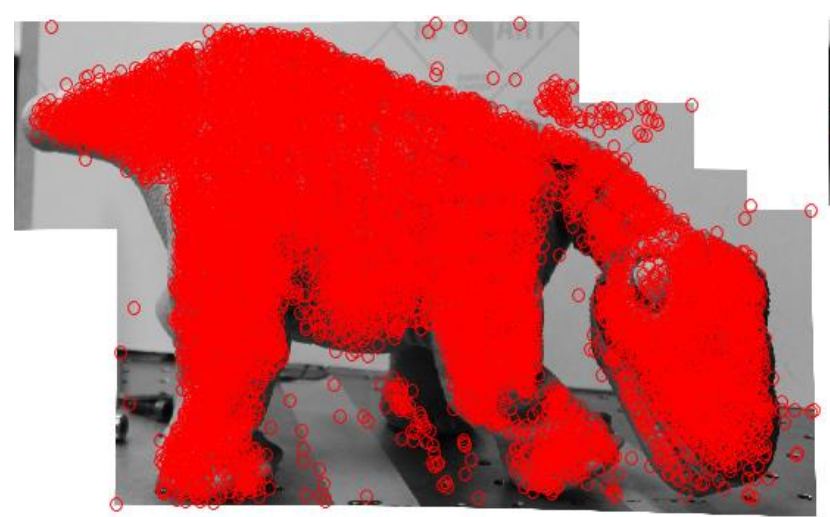

(a)

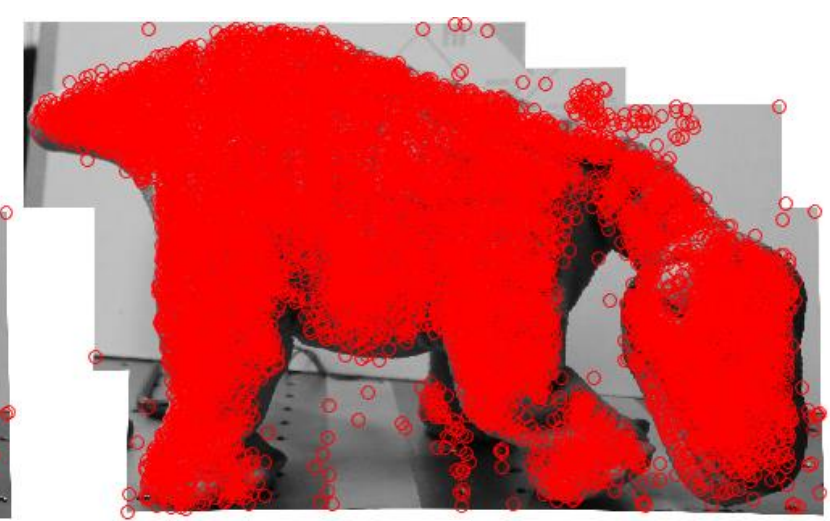

(b)

Figure E.3: Matched SIFT features, left image (a), right image (b). 


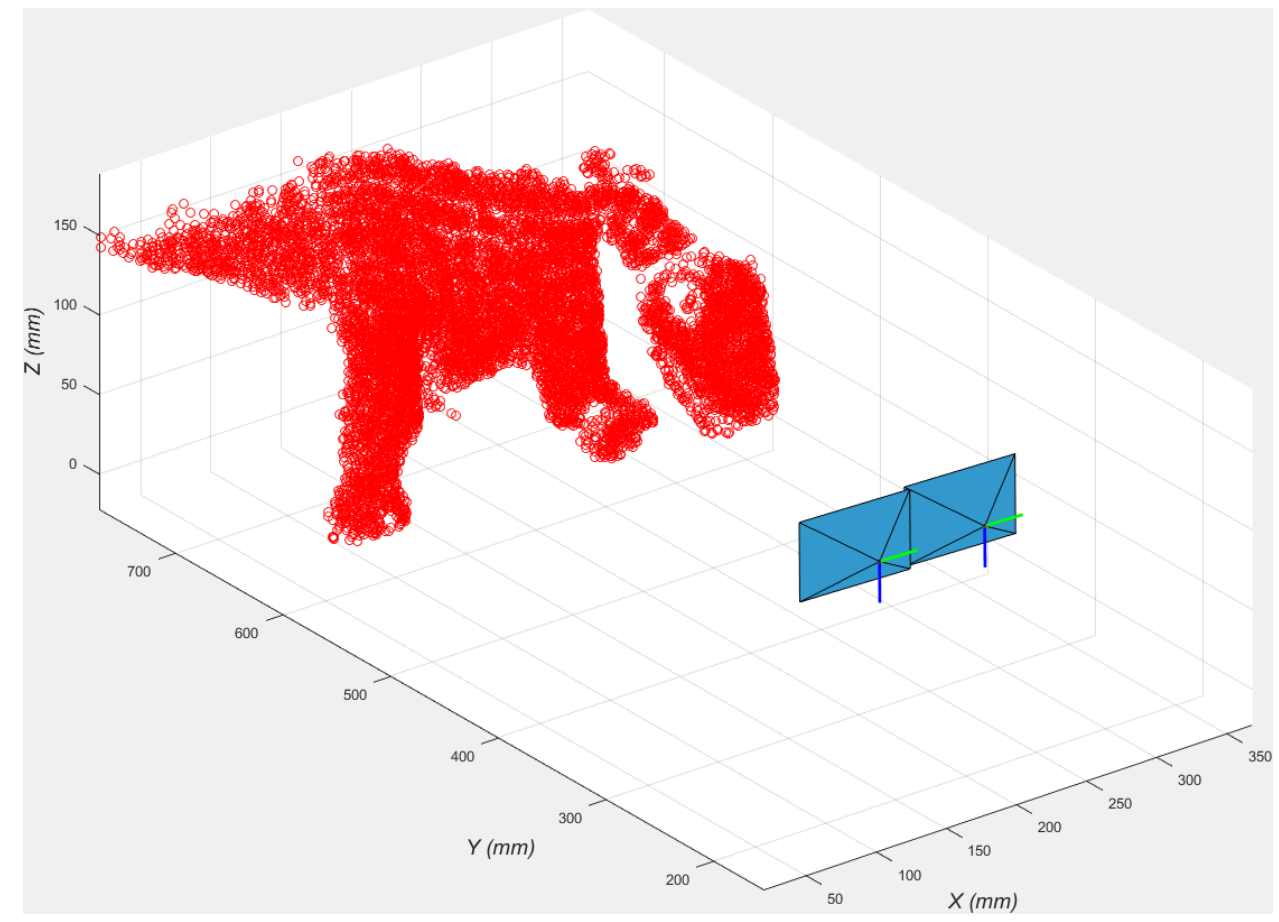

Figure E.4: Resulting triangulation point cloud, isometric view.

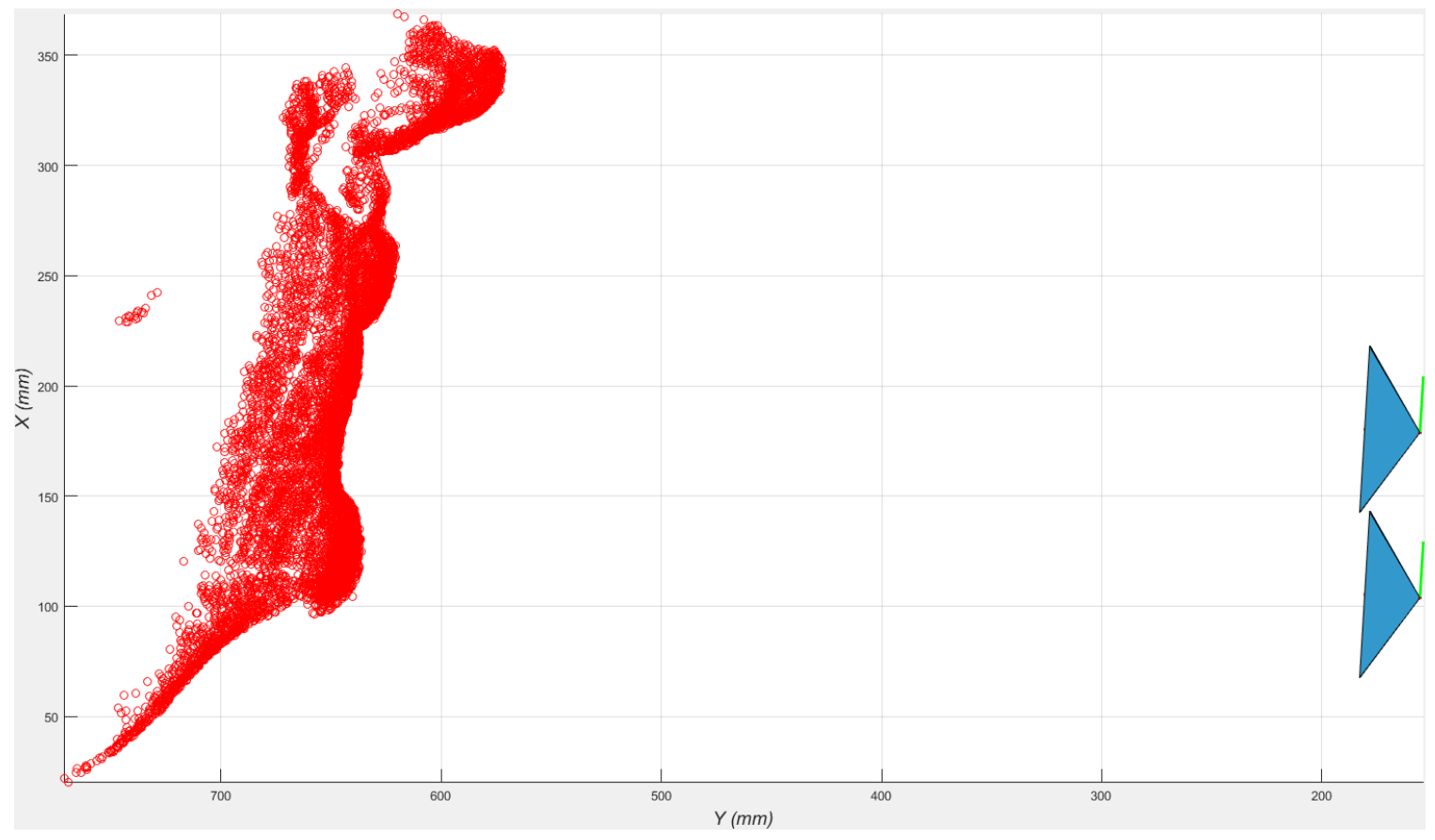

Figure E.5: Resulting triangulation point cloud, top view. 


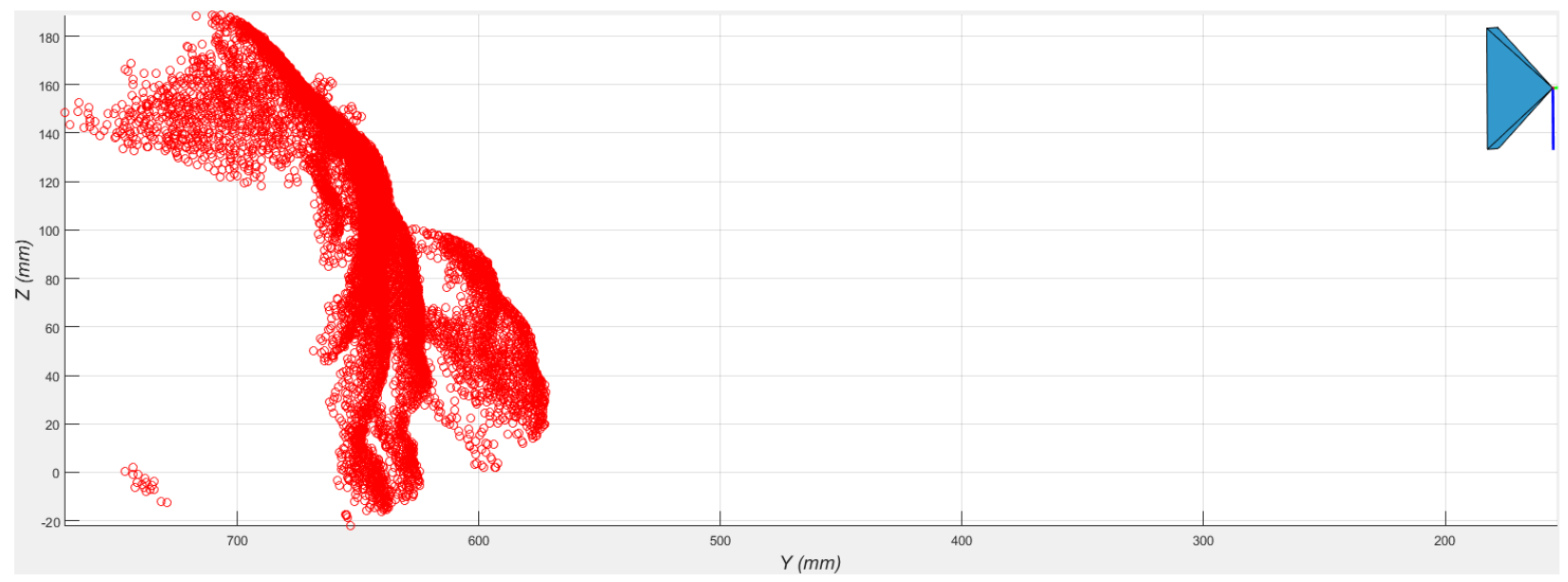

Figure E.6: Resulting triangulation point cloud, front view. 


\section{Appendix F}

The following figures illustrate movie-strip representations of the captured data from the experiments. The left-most column represents the recovered surface patches, the second column represents the recovered point cloud at the demand instant, and the third column represents the tracking to prediction offsets. 


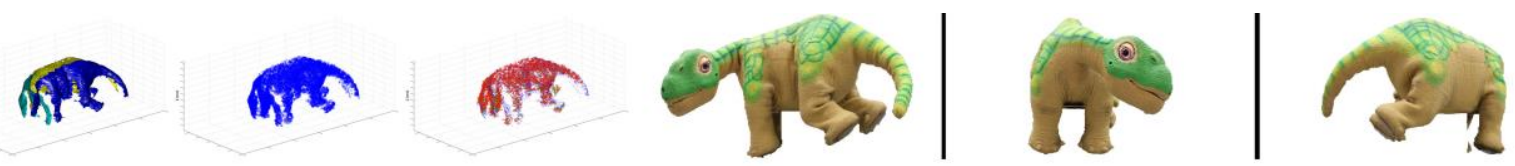

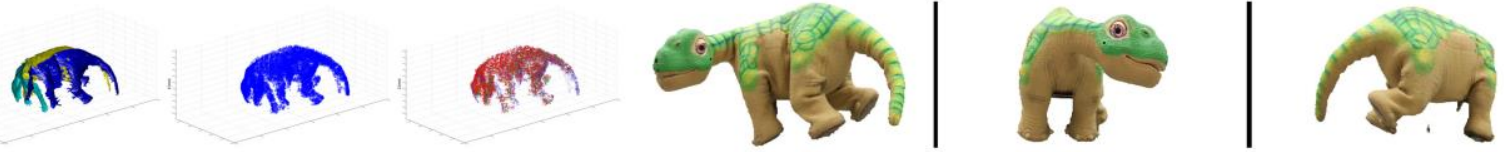

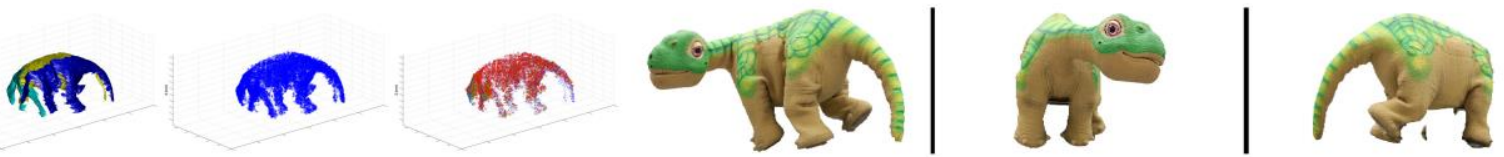

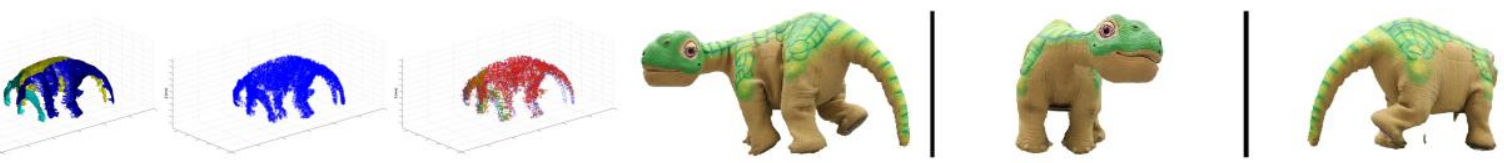

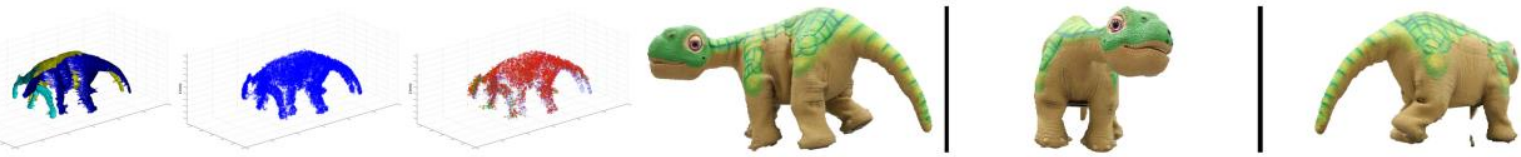

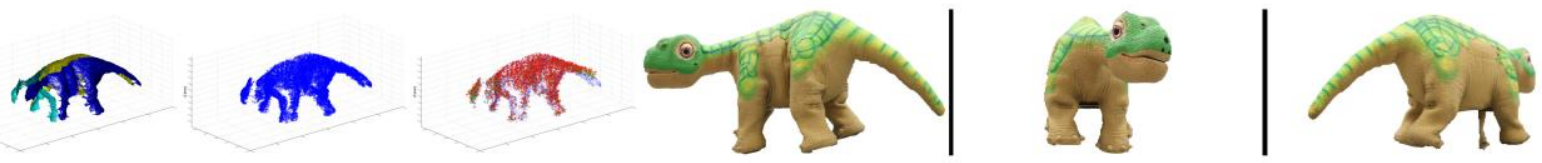

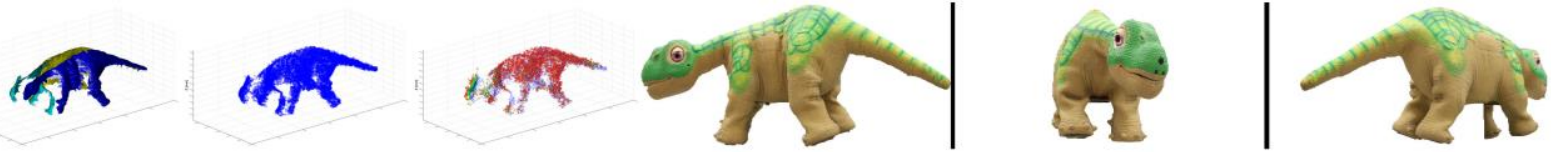

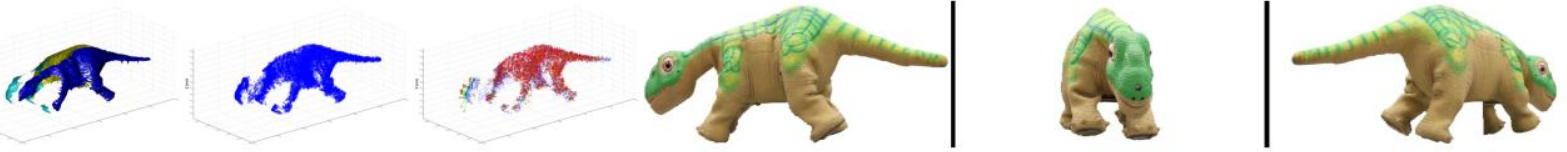

कर

Figure F.1: Movie-strip for Experiment 1. 


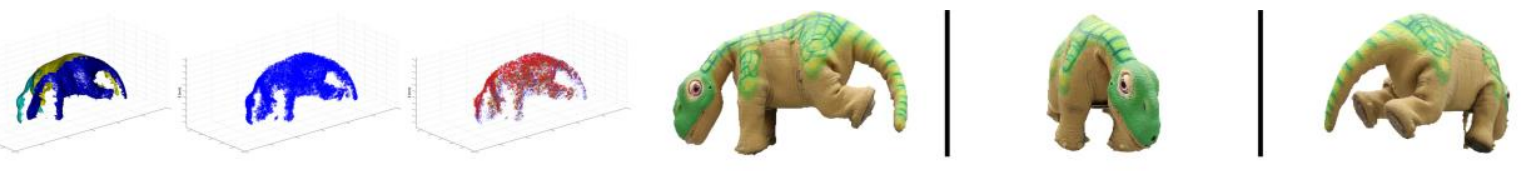

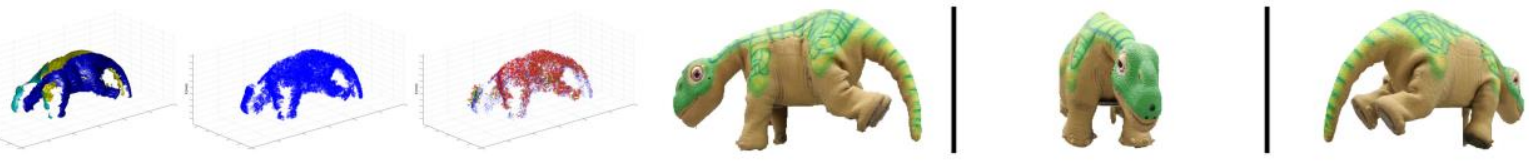

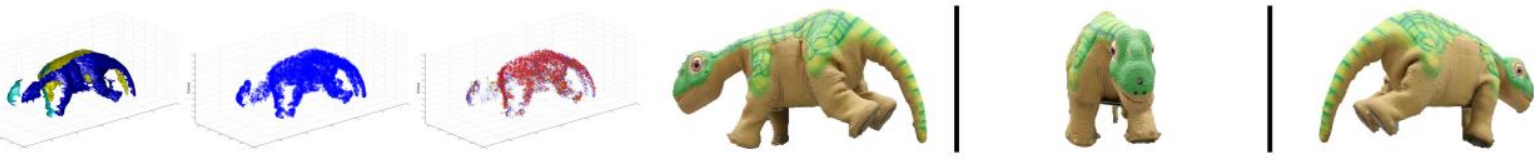

an ares

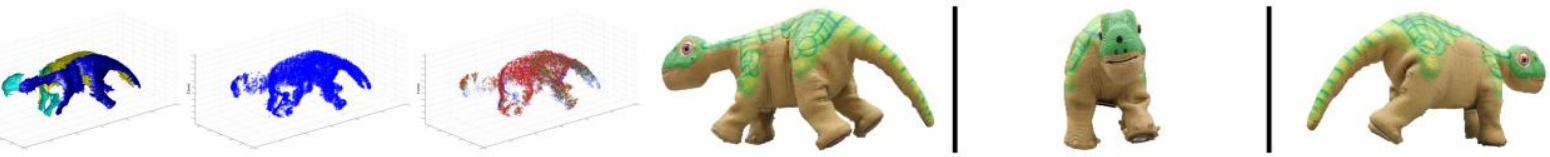

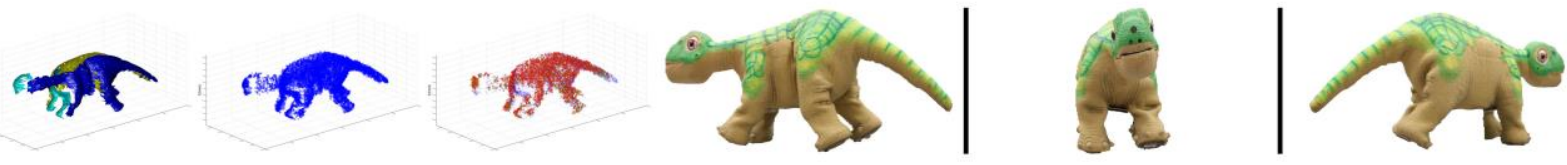

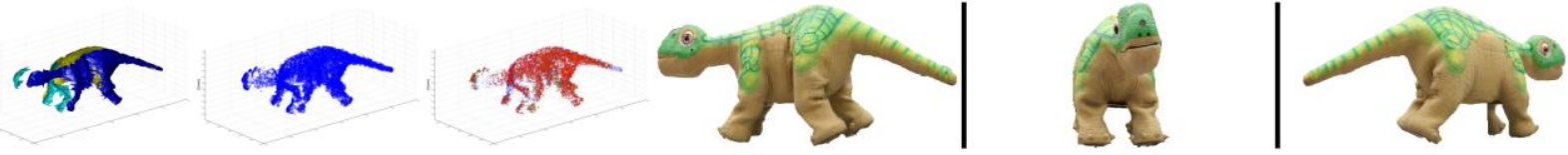

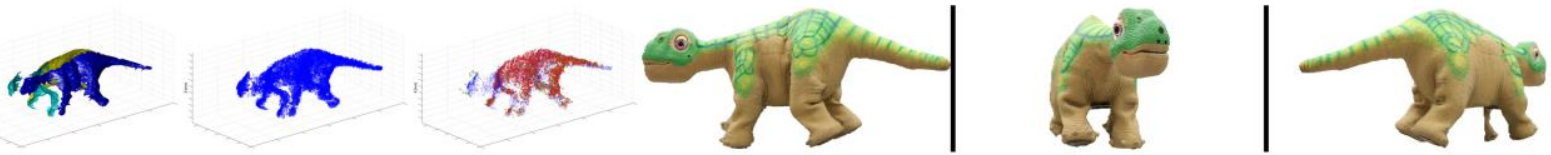

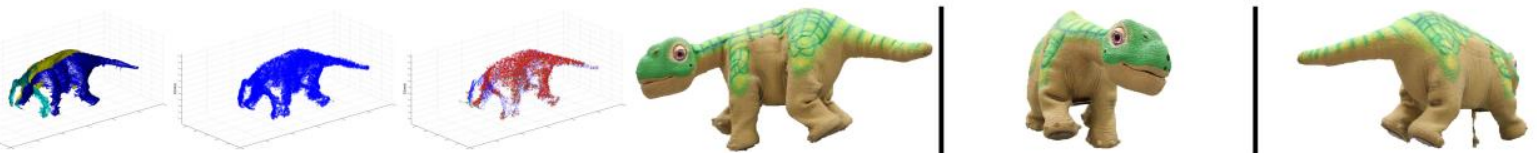

Figure F.2: Movie-strip for Experiment 2. 


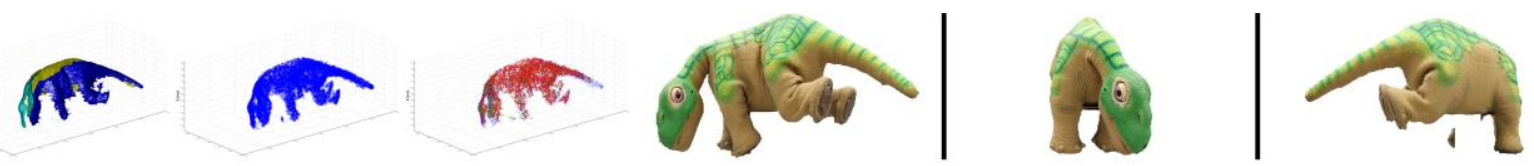

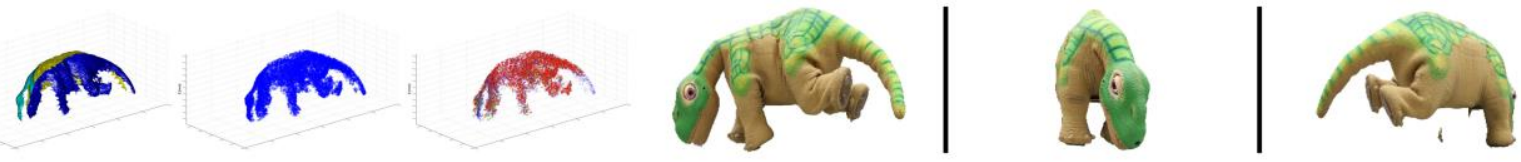

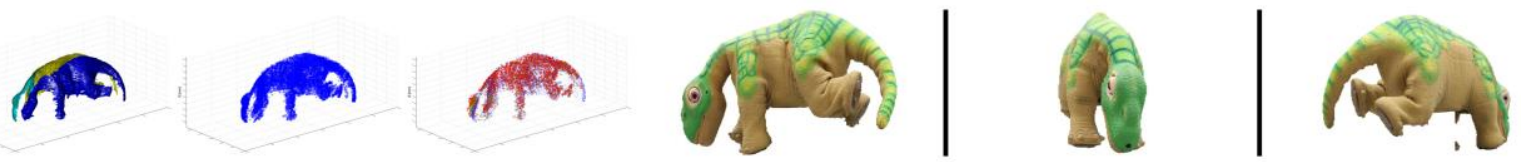

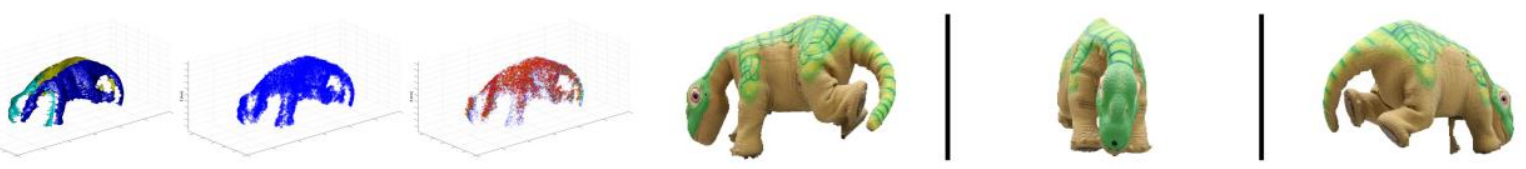

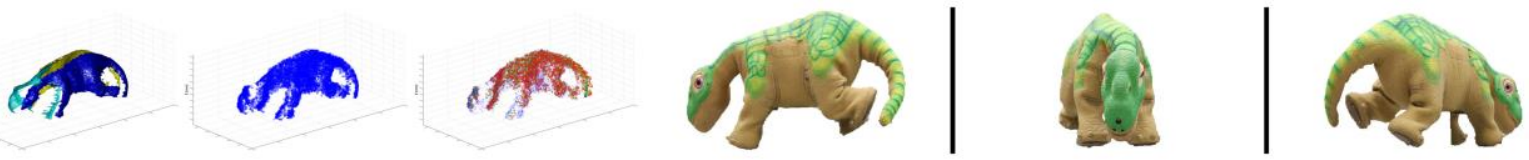

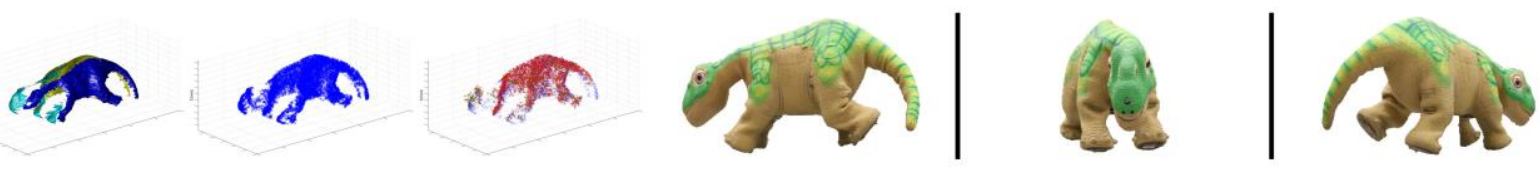

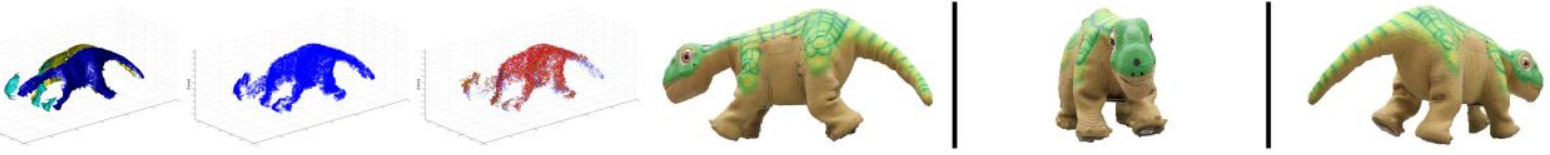

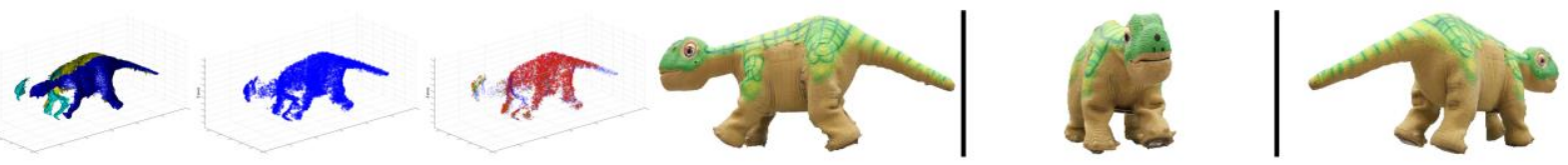

का या काष

Figure F.3: Movie-strip for Experiment 3. 


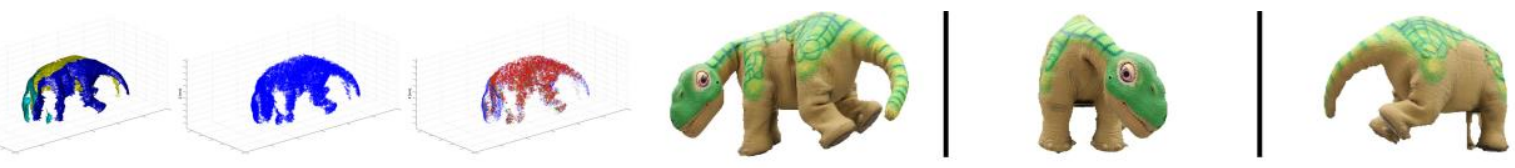

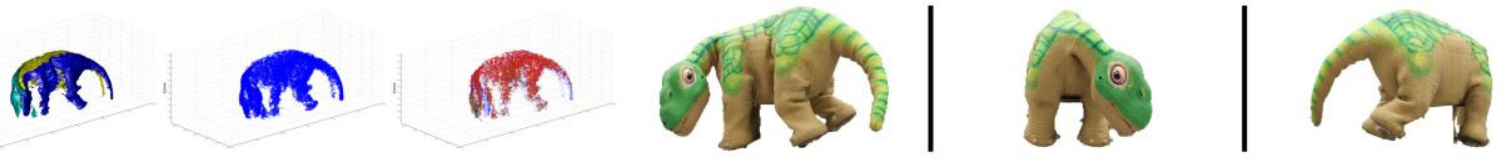

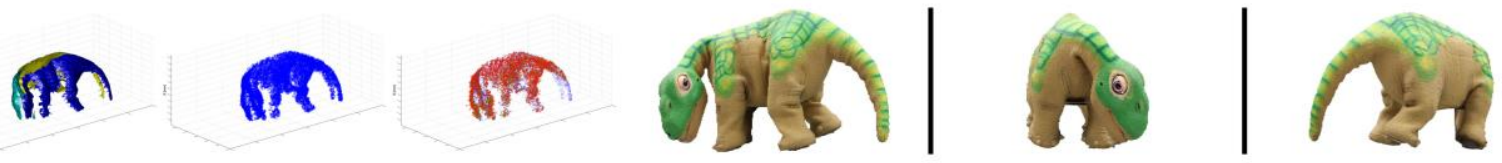

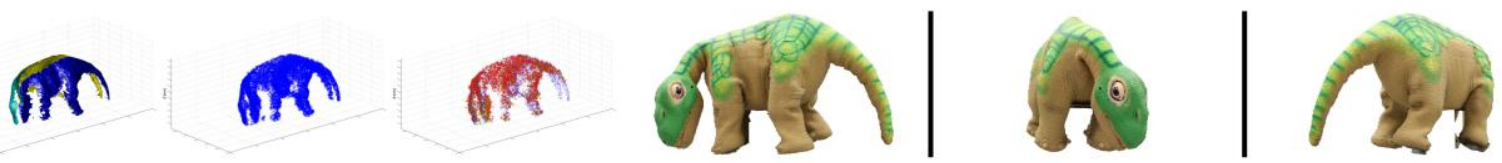

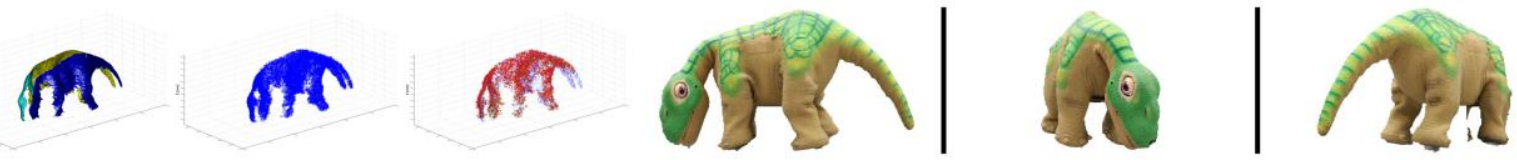

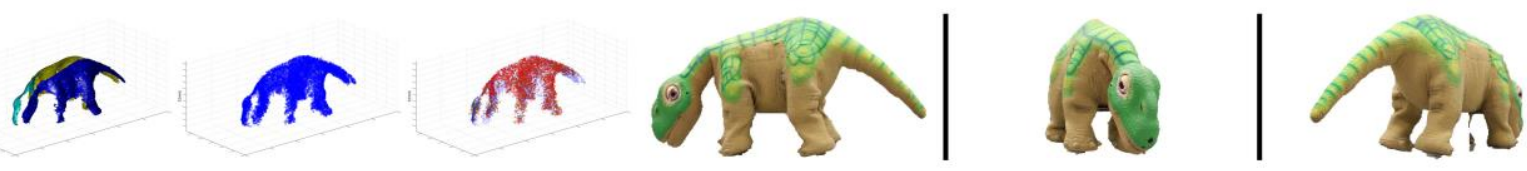

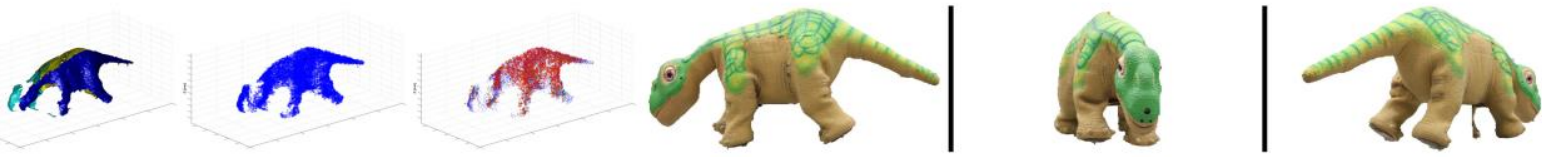

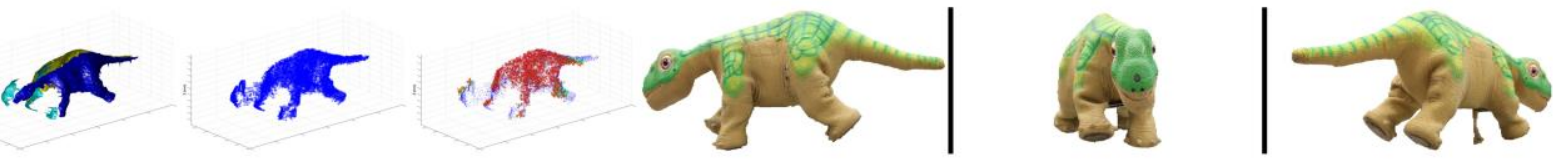

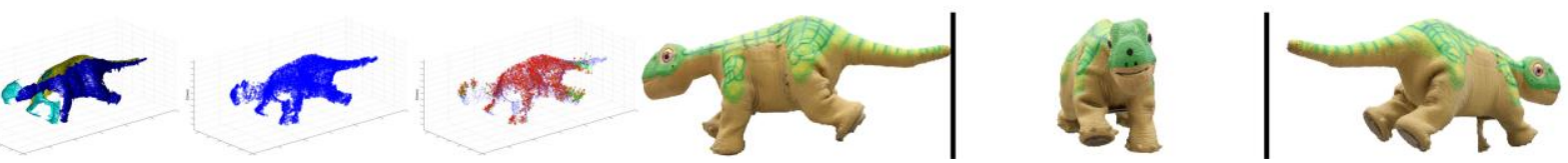

Figure F.4: Movie-strip for Experiment 4. 


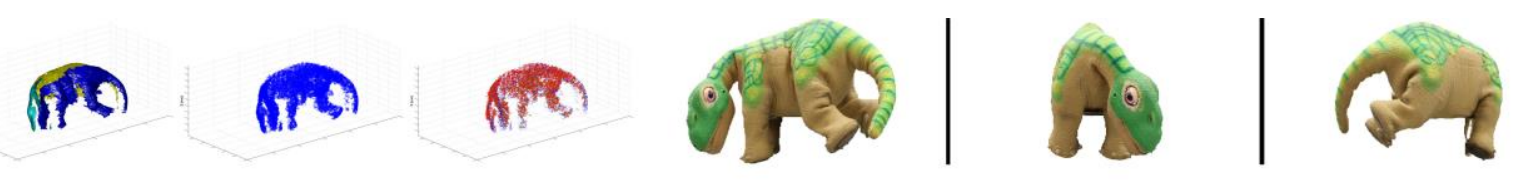

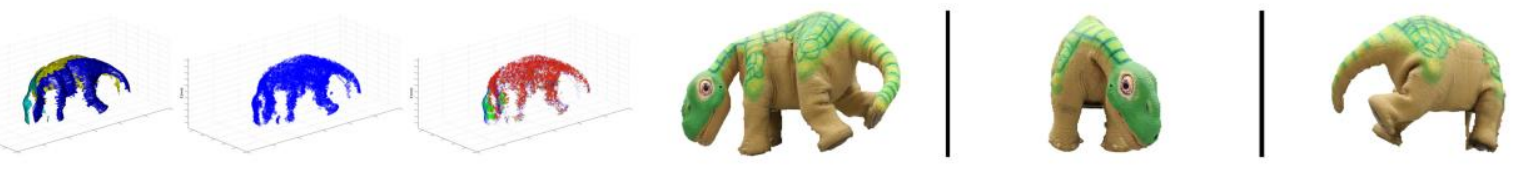

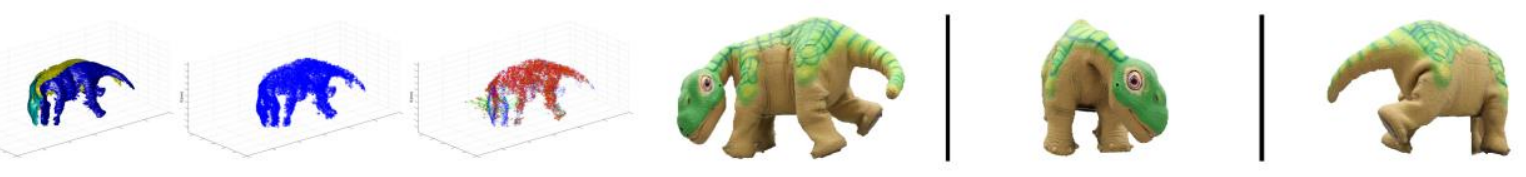

ก म भाष

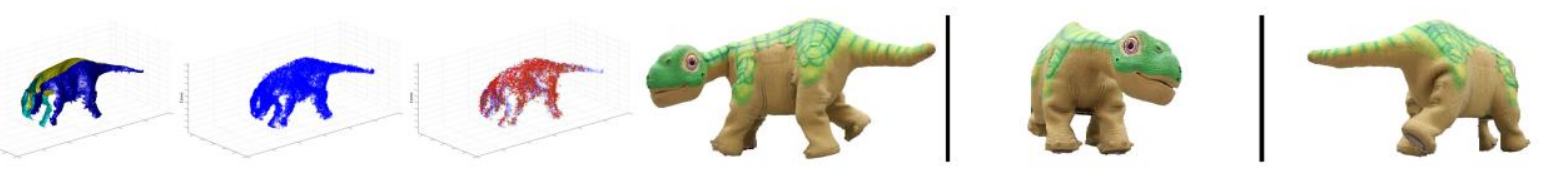

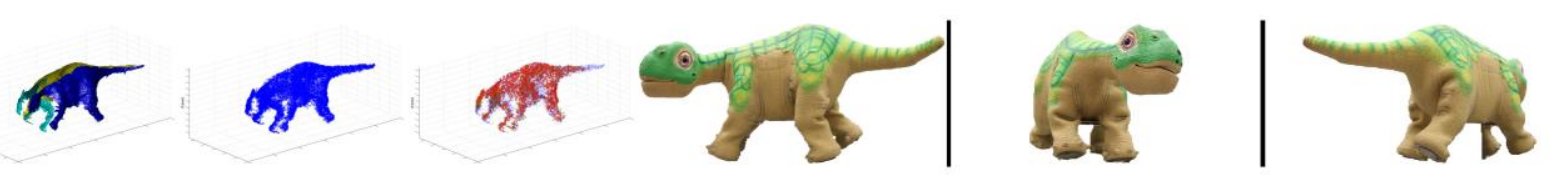

a m

a $\pi$ -

$\pi$ ari

Figure F.5: Movie-strip for Experiment 5. 


\section{Appendix G}

Supplementary figures of odd demand instants of both reconfigurable and static cameras for a single experiment with all model polygons filled. Green polygons represent stereo visible surface area, red indicate not visible through stereo. 

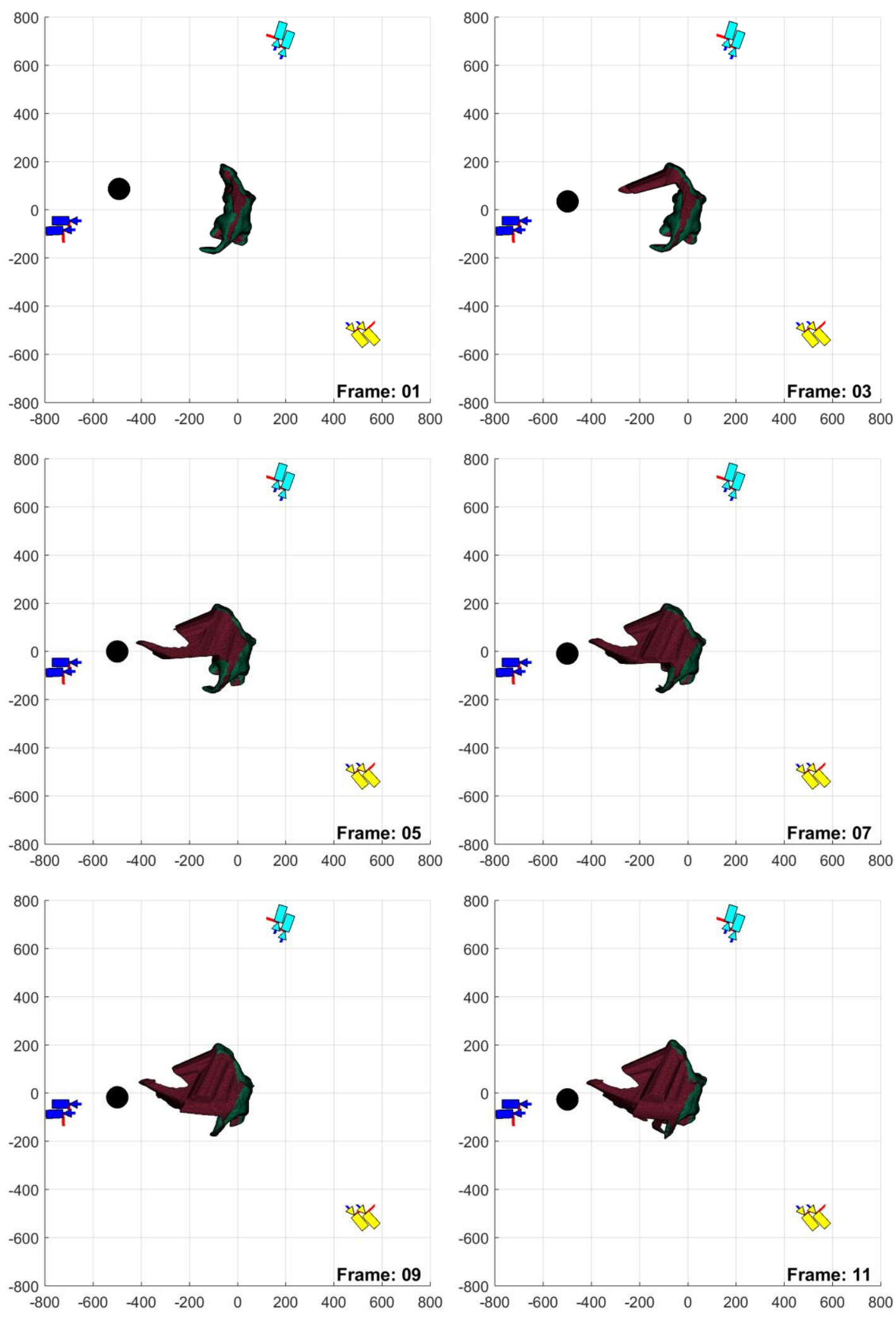

Figure G.1: Static camera experiment, Simulation Frames $\{1,3,5,7,9,11\}$, respectively. 

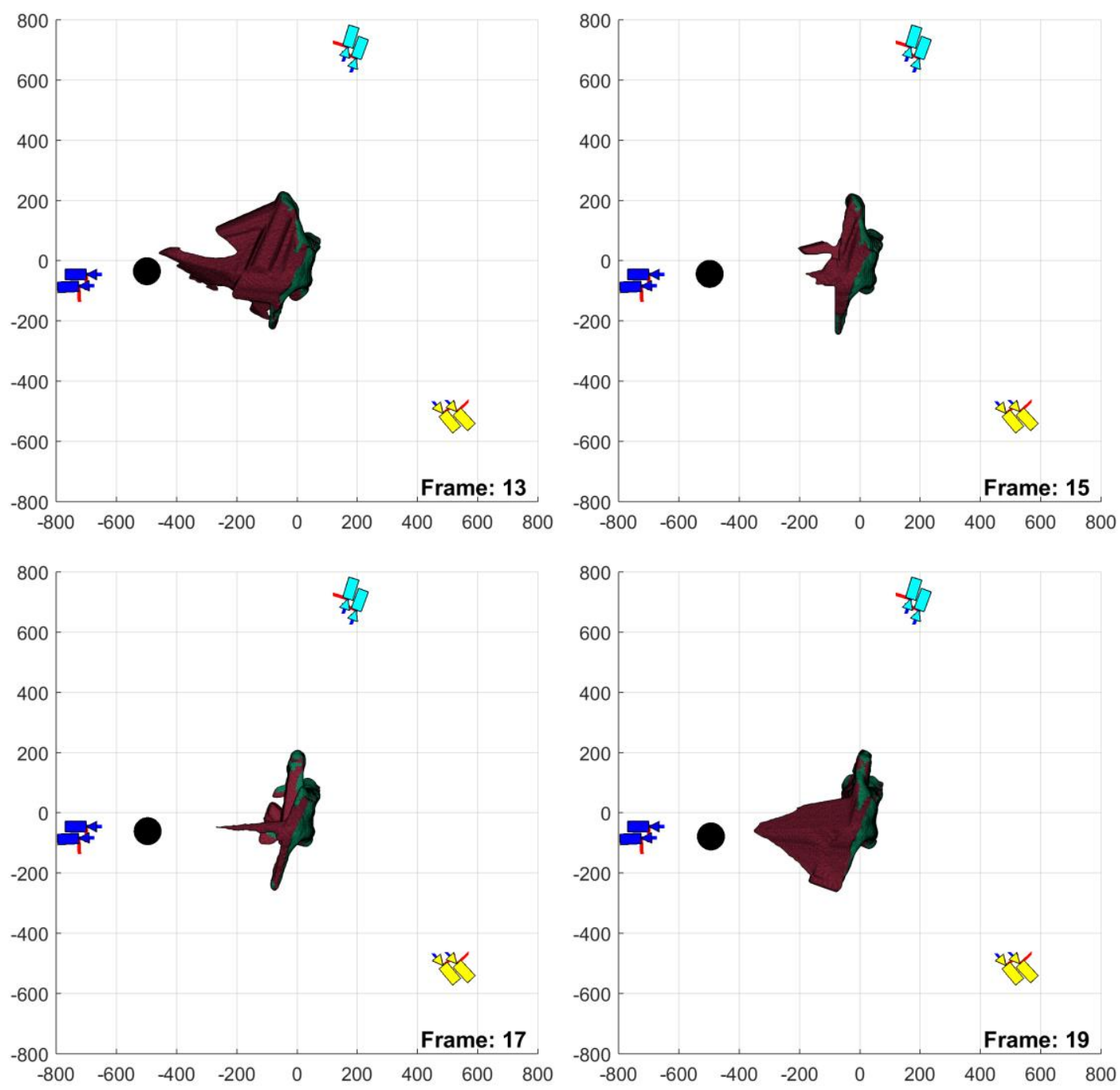

Figure G.2: Static camera experiment, Simulation Frames $\{13,15,17,19\}$, respectively. 

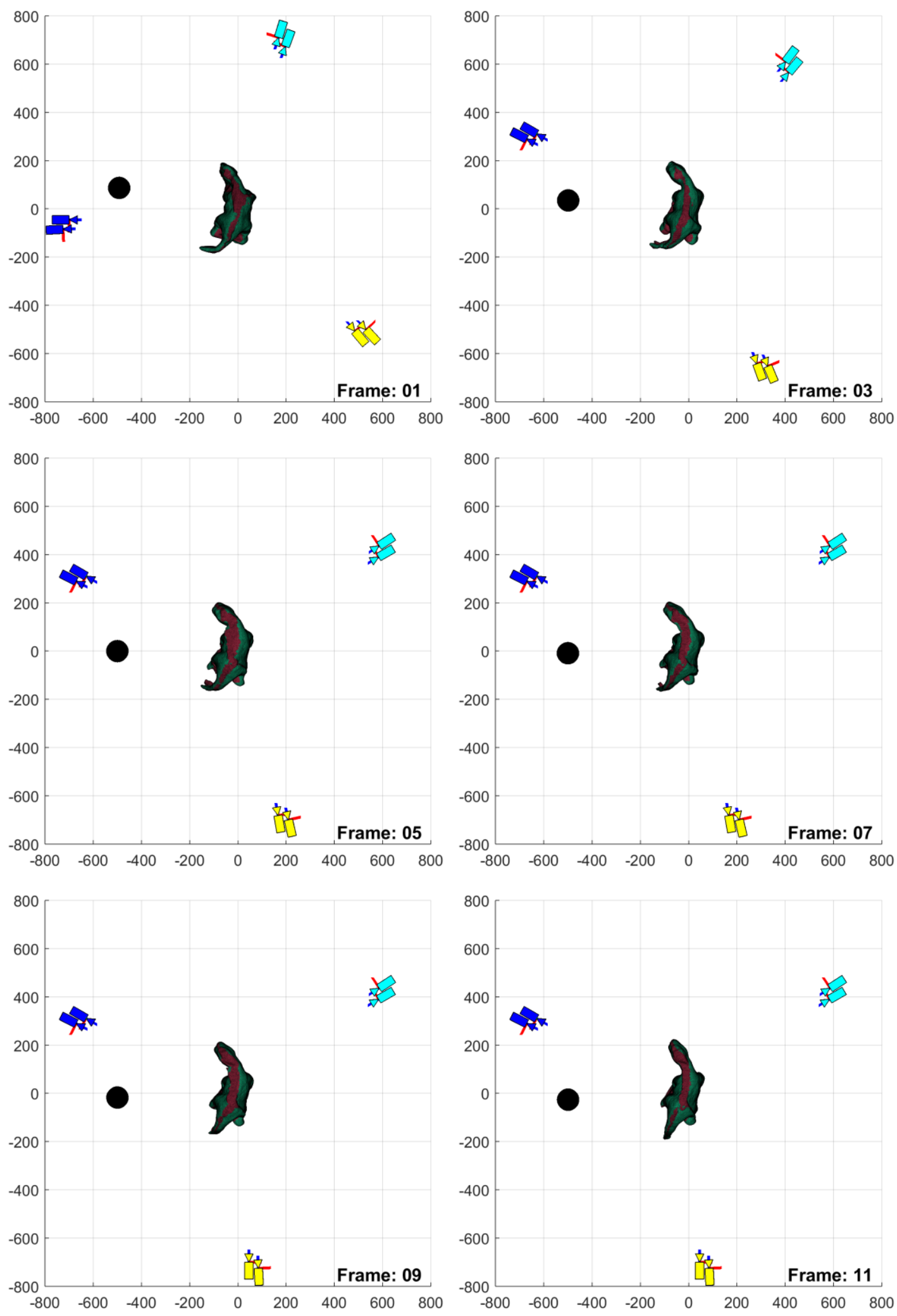

Figure G.3: Reconfiguration experiments through proposed method, Simulation Frames $\{1,3,5,7,9$, $11\}$, respectively. 

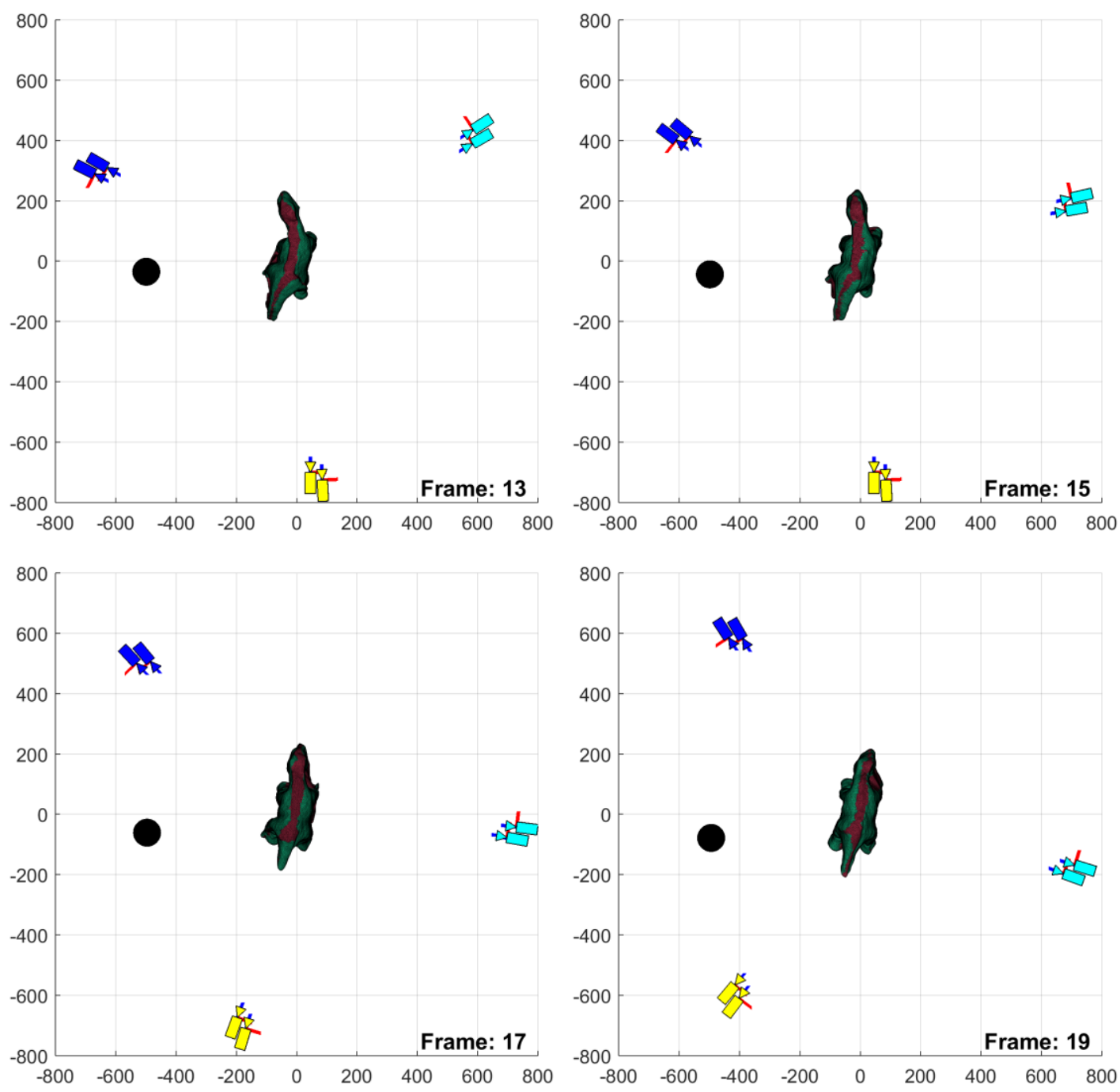

Figure G.4: Reconfiguration experiments through proposed method, Simulation Frames $\{13,15,17,19\}$, respectively. 